\title{
Sources of Particulate Matter Emissions Variability from a Gasoline Direct InJeCtion Engine
}

by

Manuel José Matos Graça Ramos

A thesis submitted in conformity with the requirements

for the degree of Master of Applied Science

Department of Mechanical and Industrial Engineering

University of Toronto

(C) Copyright 2015 by Manuel José Matos Graça Ramos 


\begin{abstract}
Sources of Particulate Matter Emissions Variability from a Gasoline Direct Injection Engine

Manuel José Matos Graça Ramos

Master of Applied Science

Department of Mechanical and Industrial Engineering

University of Toronto
\end{abstract}

2015

Ultrafine particulate matter (PM) emissions from gasoline direct injection engines are a concern due to their associated health effects. This experimental study investigated sources of PM emissions variability observed in previous work, along with the effect of ethanol content in gasoline on PM emissions. Engine operating parameters and test conditions were studied and controlled, but could not account for the level of variability observed. FTIR measurements of gas phase hydrocarbon emissions provided evidence that fuel composition changes were responsible for this variability. Exhaust emissions of toluene and isobutylene were interpreted as markers of gasoline aromatic content and gasoline volatility, respectively. Tests with gasoline containing added toluene (10\%) supported this hypothesis and showed that the PM emissions variability can be attributed to changes in the composition of the pump gasoline. Tests with gasoline containing added ethanol (10\% and 30\%) increased PM emissions at steady-state operation and decreased emissions during engine start-up. 


\section{Acknowledgements}

First and foremost, I'd like to thank Professor James S. Wallace for providing me with this wonderful opportunity. He was always there to provide useful guidance and remained enthusiastic and optimistic, even when things went awry. These past two years have been very formative for me, both personally and professionally, and they will remain a treasured memory.

Secondly, I'd like to acknowledge my predecessor Phillip Mireault, who worked with me for over a year on this project. He was always willing to teach me new things and quick to respond to my questions, even after his graduation. Without a doubt, his meticulous work provided me with an excellent foundation for my thesis. A special thank you also goes to Naomi Zimmerman, who helped immensely with the processing of EC/OC filters. She never failed to provide her near bottomless expertise on sampling instruments, as well as digress into discussions on statistics and their greater meaning.

The helpful assistance provided by both Osmond Sargeant and Terry Zak cannot go unacknowledged. Their technical expertise in all things mechanical and electrical allowed my work to proceed, and their

patience in explaining things to me was truly appreciated. The fine gentlemen in the machine shop in MC120 were likewise always helpful and willing to lend a hand when asked.

I'd also like to thank my ERDL lab-mates, in particular Dan, Robert, Vahid, Khaled, Sean, Ivan, and Alin. They were always able to provide helpful assistance when asked, and they provided for a great atmosphere that made working at the ERDL a pleasure.

Finally I'd like to thank my family and my darling Alexis for their continued love and support as I pursued my academic education. Their belief in me kept me going even when things were seemingly insurmountable. 


\section{Contents}

1 Introduction $\quad 1$

1.1 Gasoline Direct Injection Overview . . . . . . . . . . . . . . . . . . . 2

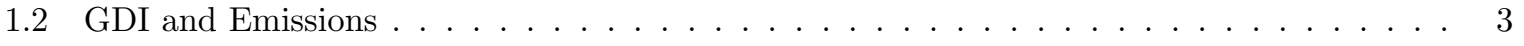

1.3 Health Effects of Particulate Matter _ . . . . . . . . . . . . . . . . 4

1.4 PM Regulations Driving Research $\ldots \ldots \ldots \ldots \ldots \ldots$

$\begin{array}{lll}2 & \text { Emissions from GDI Engines } & \mathbf{7}\end{array}$

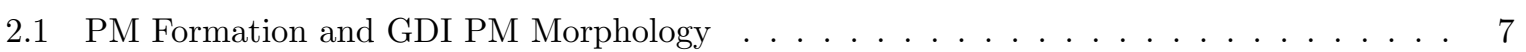

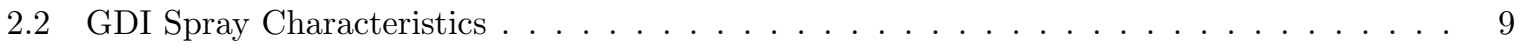

2.3 GDI Engine Parameters and $\mathrm{PM} \ldots \ldots \ldots \ldots \ldots \ldots \ldots$

2.3.1 Engine Coolant Temperature . . . . . . . . . . . . . . . . . 10

2.3 .2 Ignition Timing . . . . . . . . . . . . . . . . . . . . . . . 12

2.3 .3 Injection Timing and Duration . . . . . . . . . . . . . . . 12

2.3 .4 Equivalence Ratio . . . . . . . . . . . . . . . . . . . . . . . 13

2.3.5 Engine Operating Condition . . . . . . . . . . . . . . . . . . . . . 14

2.3 .6 Valve Timing . . . . . . . . . . . . . . . . . . . . 15

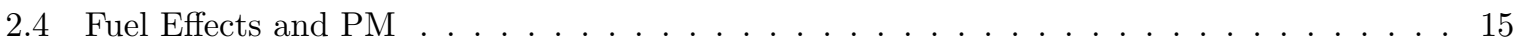

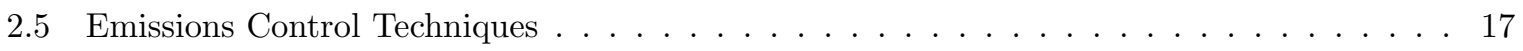

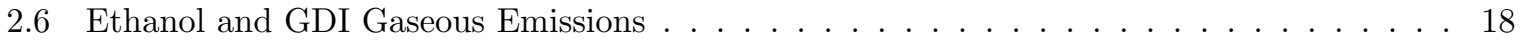

2.7 Sampling Techniques and Considerations . . . . . . . . . . . . . . . . . . . . . 19

3 Motivation and Experimental Goals $\quad 22$

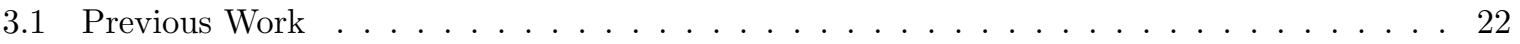

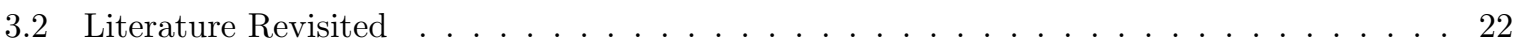

3.2 .1 Dilution Air Humidity . . . . . . . . . . . . . . . 23

3.2 .2 Engine Deposit Formation . . . . . . . . . . . . . . . . . . . 24

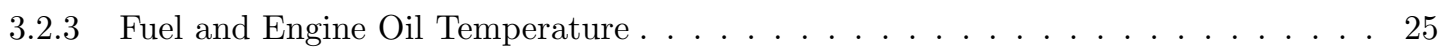

3.2 .4 Engine Oil Age and Fuel Dilution . . . . . . . . . . . . . . . . . . 25

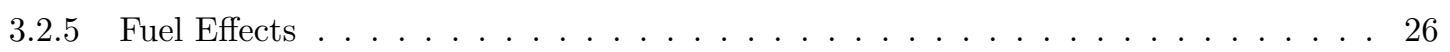

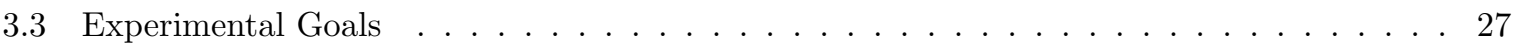

4 Experimental Set-up $\quad 28$

4.1 Research Engine . . . . . . . . . . . . . . . . . . . . . 28

4.1 .1 Powertrain Control Module . . . . . . . . . . . . . . . . . . 28 
4.1 .2 Engine Exhaust System _ . . . . . . . . . . . . . . . . . . . . . 29

4.1 .3 Fuelling System . . . . . . . . . . . . . . . . . . . . . . . 29

4.1 .4 Dynamometer . . . . . . . . . . . . . . . . . . . . 30

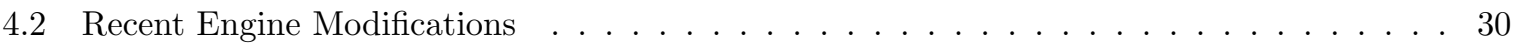

4.2 .1 Fuel Cooler . . . . . . . . . . . . . . . . . . . . . . . . . . . 30

4.2 .2 Oil Cooler . . . . . . . . . . . . . . . . . . . . . 30

4.2 .3 Crankcase Ventilation Filter . . . . . . . . . . . . . . . . . . 31

4.3 Engine Controls . . . . . . . . . . . . . . . . . . . . . . . . . . . . 33

4.3 .1 Throttle Control . . . . . . . . . . . . . . . . . . . 33

4.3 .2 Dynamometer Control . . . . . . . . . . . . . . . . . . . . . 34

4.4 Engine Data Acquisition and Control . . . . . . . . . . . . . . . . . . 34

4.4 .1 National Instruments Compact DAQ . . . . . . . . . . . . . . . . . 34

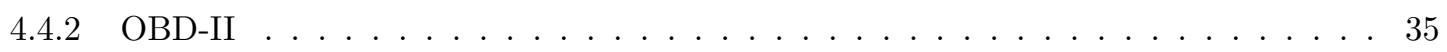

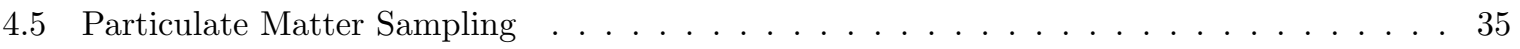

4.5.1 TSI Rotating Disk Thermodiluter _ . . . . . . . . . . . . . . . 35

4.5 .2 Dekati FPS 4000 Diluter . . . . . . . . . . . . . . . . 36

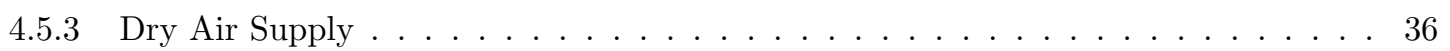

4.5.4 Engine Exhaust Particle Sizer . . . . . . . . . . . . . . . . . 36

$4.5 .5 \quad$ PM Filter Collection Cart . . . . . . . . . . . . . . . . 37

4.5.6 Gravimetric Filter Analysis . . . . . . . . . . . . . . . . . . 37

4.5 .7 Compositional Filter Analysis . . . . . . . . . . . . . . 37

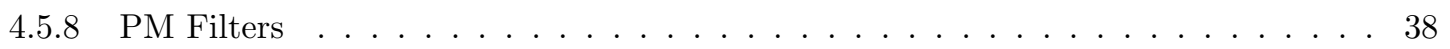

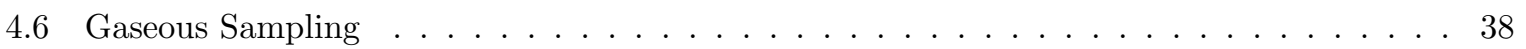

4.6 .1 Fuel-Air Equivalence Ratio . . . . . . . . . . . . . . . . . 38

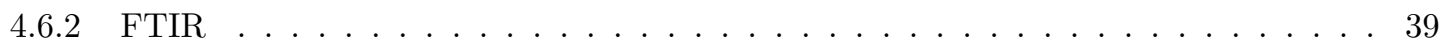

4.6 .3 Standard Emissions Bench . . . . . . . . . . . . . . . . . . . . . 39

4.6 .4 Mini $\mathrm{CO}_{2}$ Monitor . . . . . . . . . . . . . . . . . . . 41

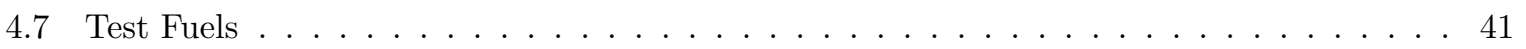

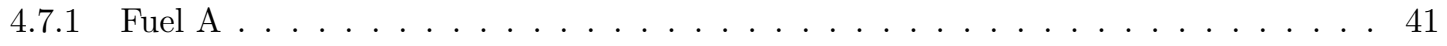

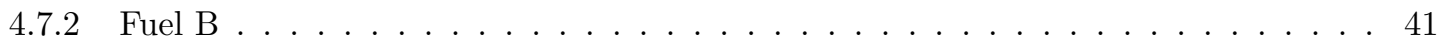

4.7 .3 Fuel Blends . . . . . . . . . . . . . . . . . . . . . . . . 42

5 Experimental Methods $\quad 46$

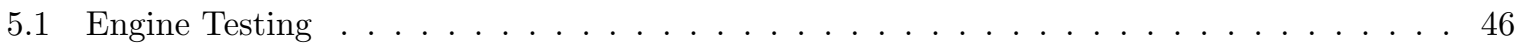

5.1 .1 Pre-Engine Test Set-up . . . . . . . . . . . . . . . . . . 46

5.1 .2 Transient Test Procedures . . . . . . . . . . . . . . . . . . . . . 47

5.1 .3 Steady-State Test Procedures . . . . . . . . . . . . . . . . . . . . 48

5.1 .4 Fuel Change-over . . . . . . . . . . . . . . . . . . . . . 50

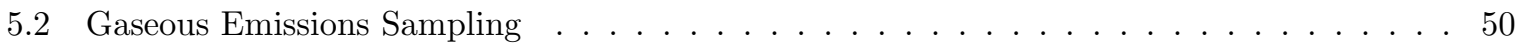

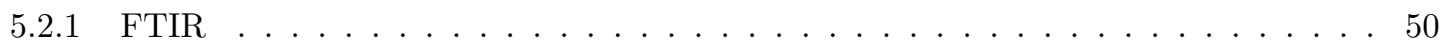

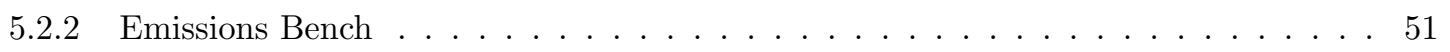

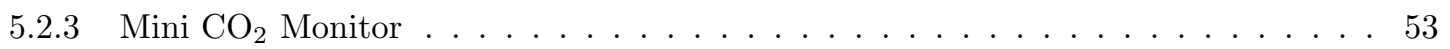

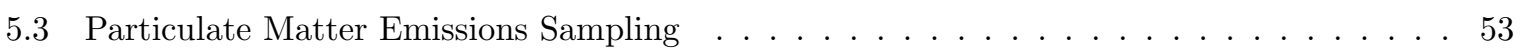

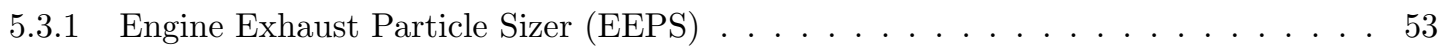


5.3 .2 Rotating Disk Thermodiluter . . . . . . . . . . . . . . . . 54

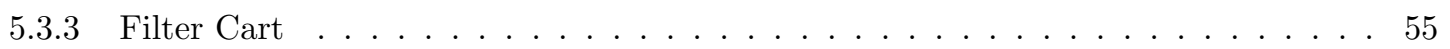

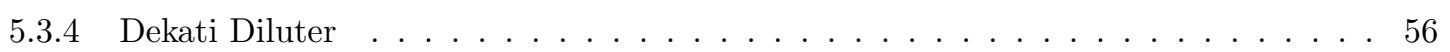

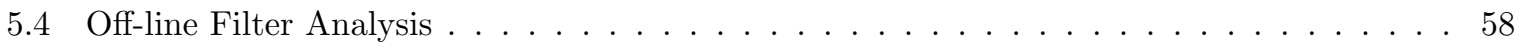

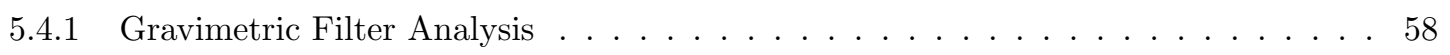

$5.4 .2 \quad$ Filter Composition Analysis . . . . . . . . . . . . . . . . . . 59

6 Steady-State Results $\quad 62$

6.1 Systematic Sources of Variability . . . . . . . . . . . . . . . . . . 65

6.1 .1 Dilution Air Humidity . . . . . . . . . . . . . . . 65

6.1 .2 Fuel Temperature . . . . . . . . . . . . . . . . . . 66

6.1 .3 Engine Deposits and Engine Cleaning . . . . . . . . . . . . . . . 69

6.1 .4 Engine PCV Filter . . . . . . . . . . . . . . . . 73

6.1.5 Engine Parameters (Re-visited) . . . . . . . . . . . . . . . . . . 74

6.1 .6 Engine Oil Age . . . . . . . . . . . . . . . . . . . . 77

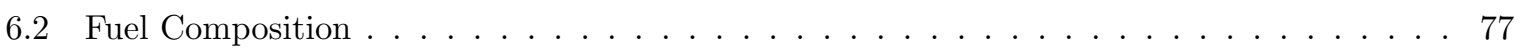

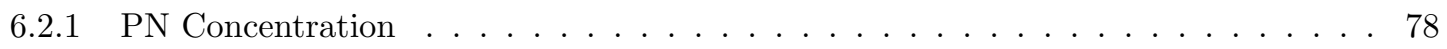

$6.2 .2 \quad \mathrm{PN}$ Size Distribution . . . . . . . . . . . . . . . . . . . . . . 82

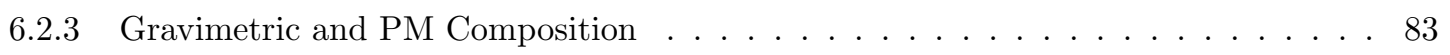

6.2 .4 Gaseous Emissions . . . . . . . . . . . . . . . . . . . . . 87

6.3 PN vs. Gaseous Species . . . . . . . . . . . . . . . . . . . . . . . 94

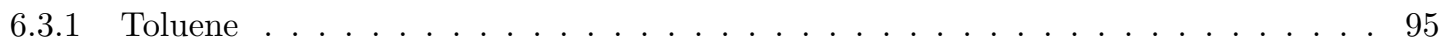

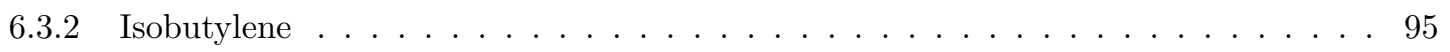

6.3 .3 Multiple Linear Regression _. . . . . . . . . . . . . . . . . . . . . 95

6.4 PN Index and Varying Fuel Properties . . . . . . . . . . . . . . . . . . . . 97

6.4 .1 Variations of Base Gasoline . . . . . . . . . . . . . . . . . 98

$6.4 .2 \quad$ PN Index Revised . . . . . . . . . . . . . . . . . . . . . . . . . . 99

6.5 Results Synthesis: Explaining the Observed Variability . . . . . . . . . . . . . . . 101

7 Transient Results $\quad 104$

7.1 PN Concentrations and Size Distributions . . . . . . . . . . . . . . . . . 104

7.1 .1 Cold Start . . . . . . . . . . . . . . . . . . . . . . . 104

$7.1 .2 \quad$ Hot Start . . . . . . . . . . . . . . . . . . . . 107

7.1 .3 PN Totals . . . . . . . . . . . . . . . . . . . . . . . . 111

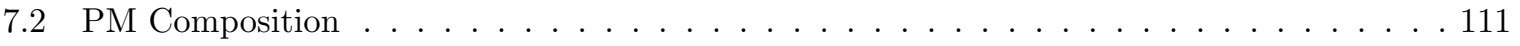

7.3 Gaseous Emissions . . . . . . . . . . . . . . . . . . . . . . . . . 114

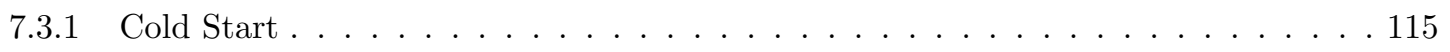

$7.3 .2 \quad$ Hot Start . . . . . . . . . . . . . . . . . . . . . 116

8 Conclusions and Recommendations 118

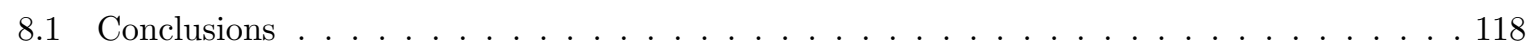

8.1 .1 Sources of PM Emission Variability . . . . . . . . . . . . . . . 118

8.1 .2 Additional Conclusions . . . . . . . . . . . . . . . . . . 120

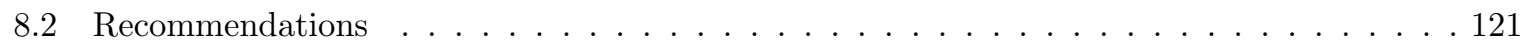


8.3 Future Work . . . . . . . . . . . . . . . . . . . . . . 122

$\begin{array}{ll}\text { References } & 124\end{array}$

A Chapter 4 Supplementary Apparatus Information 132

A.1 Pro-Vent 200 Modification . . . . . . . . . . . . . . . . . . . . . . . . 132

A.2 Atomic Ratio Calculations . . . . . . . . . . . . . . . . . . . . 134

A.2.1 Ethanol Content Regression . . . . . . . . . . . . . . . . . . . . 134

A.2.2 Atomic Ratio Calculations for Toluene Blends . . . . . . . . . . . . . . 136

A.3 Dry Air Supply . . . . . . . . . . . . . . . . 136

B Chapter 5 Calculations $\quad 137$

B.1 Road Load Calculations . . . . . . . . . . . . . . . . . . . . . . 137

B.2 Emissions Bench Oxygen Correction . . . . . . . . . . . . . . . . . 138

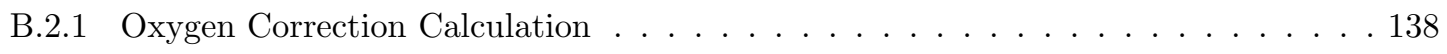

B.2.2 Oxygen Correction Factors . . . . . . . . . . . . . . . . 139

B.3 Dilution Ratio Calculation . . . . . . . . . . . . . . . . . . . . 139

C Chapter 6 Supplementary Notes, Discussions, and Calculations 141

C.1 Engine Cleaning Procedure . . . . . . . . . . . . . . . . . . . . 141

C.2 Fuel Trim Drift: Marker for Temporal Variations . . . . . . . . . . . . . . . . . . . . . . . . . . . . . . . .

C.3 Exhaust Temperature: Marker for Combustion Stability . . . . . . . . . . . . . . . 143

C.4 Engine Oil Dilution: Marker for Wall Impingement . . . . . . . . . . . . . . . . . 145

C.5 FTIR vs. Emissions Bench . . . . . . . . . . . . . . . . . . . 146

C.6 PN Index Calculation . . . . . . . . . . . . . . . . . . . . . . . 148

C.6.1 Fuel Properties Required for PN Index . . . . . . . . . . . . . . . . . . . . . . . . . . . . . . . . . . . . . . .

C.6.2 Calculation of PN Index . . . . . . . . . . . . . . . . . 148

C.7 PN Index Revised . . . . . . . . . . . . . . . . . . . . . 150

C.7.1 Fuel Properties for Revised PN Index . . . . . . . . . . . . . . . . . 150

C.7.2 Calculation of Revised PN Index . . . . . . . . . . . . . . . . 151

D Chapter 7 Supplementary Start Data 152

D.1 Cold Start: Particle Size Distributions . . . . . . . . . . . . . . . . . . . . 152

D.2 Cold Start: Gaseous Emissions _ . . . . . . . . . . . . . . . . . 153

D.3 Hot Start: Particle Size Distributions . . . . . . . . . . . . . . . . . 158

D.4 Hot Start Gaseous Emissions . . . . . . . . . . . . . . . . . . 159 


\section{List of Tables}

4.1 Research engine specifications. . . . . . . . . . . . . . . . . . . . 29

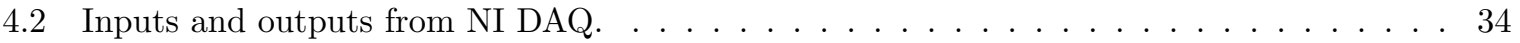

4.3 PIDs recorded from OBD-II data stream. . . . . . . . . . . . . . . . . 35

4.4 Filter specifications for both gravimetric and EC/OC analysis. . . . . . . . . . 38

4.5 Atomic ratios for the different fuel blends. . . . . . . . . . . . . . . . . . . . . . . . 39

4.6 Standard emissions analyzers, their ranges and calibrations. . . . . . . . . . . . . . 40

5.1 Cold start engine parameters and set-points. . . . . . . . . . . . . . . . . . . 47

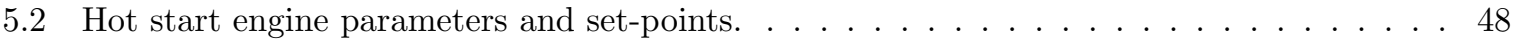

5.3 Throttle ramp from idle up to highway cruise conditions. . . . . . . . . . . . . . . 49

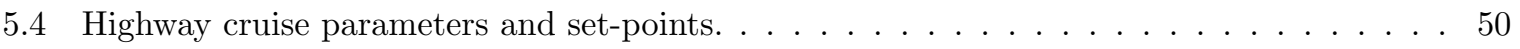

5.5 Compounds used in the FTIR method. . . . . . . . . . . . . . . . . . . . 51

5.6 Diluter box settings. . . . . . . . . . . . . . . . . . . . . . . . 54

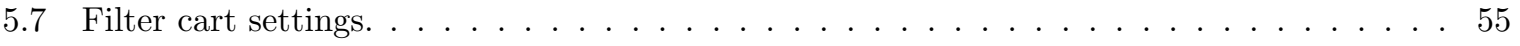

5.8 Filter collection durations. . . . . . . . . . . . . . . . . . . . . . 56

5.9 Dekati diluter DR and DT set-points and typical conditions. . . . . . . . . . . . . . 57

5.10 Dekati diluter heater ramps. . . . . . . . . . . . . . . . . . . 57

6.1 Investigation test matrix, including run breakdown of test details and instruments used. . 63

6.2 Modal and median diameters (nm) for particle size distributions for different fuel blends. . 83

6.3 Calculated PN emissions index using a variation on the procedure from Leach et al. . . . 98

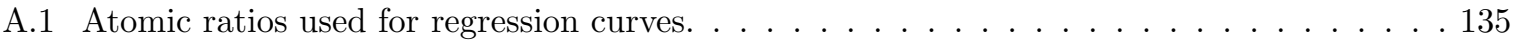

A.2 Chemical constituents and corresponding atomic ratios for the pure fuel components. . . 136

A.3 Details of the dry air supply used by the diluters in this study. . . . . . . . . . . 136

B.1 Road load engine and vehicle parameters. . . . . . . . . . . . . . . . 137

C.1 Average specific exhaust temperature and corresponding 95\% confidence intervals for each fuel blend. . . . . . . . . . . . . . . . . . . . . . . . . 145

C.2 Fuel properties for the three fuel constituents used. . . . . . . . . . . . . . . . . . 148

C.3 Calculated PN indices. . . . . . . . . . . . . . . . . . . . . . . . . . 149

C.4 Base gasoline fuel properties used for the corrected PN indices. . . . . . . . . . . . . 150

C.5 Calculated PN indices. . . . . . . . . . . . . . . . . . . . . . . 151 


\section{List of Figures}

1.1 Schematic representation of different injection systems in GDI engines. . . . . . . . . . 3

1.2 PM size comparison (courtesy of the US EPA). . . . . . . . . . . . . . . 5

2.1 PM formation mechanisms. . . . . . . . . . . . . . . . . . . . . . 8

2.2 Collected PN concentrations with engine load data from some reviewed sources. . . . . . . 11

2.3 Lines of constant ethanol content for PN concentrations vs. engine load. . . . . . . . . . 16

3.1 Two-minute average PN concentrations measured at the start, middle, and end of each run over 17 days. . . . . . . . . . . . . . . . . . . . . . . . . . . . . . 23

4.1 Research engine and emissions sampling arrangement. . . . . . . . . . . . . . . . . . 28

4.2 Fuelling system flow diagram. . . . . . . . . . . . . . . . . . . . . . . . . 29

$4.33 \mathrm{D}$ render of fuel cooler. . . . . . . . . . . . . . . . . . . . . . . . . 31

4.4 Cross-sectional view of fuel cooler. . . . . . . . . . . . . . . . . . . . . . . . . 32

4.5 Schematic of oil cooler. . . . . . . . . . . . . . . . . . . . . . . 43

4.6 Collected oil in the intake manifold. . . . . . . . . . . . . . . . . . . . . . . . . . . . . . 44

4.7 Exploded view of the MANN+HUMMEL ProVent200 oil separator filter. . . . . . . . . . 44

4.8 Filter cart flow diagram. . . . . . . . . . . . . . . . . . . . . . . 45

5.1 Typical engine test procedure. . . . . . . . . . . . . . . . . . . . . 46

5.2 GDI EC/OC thermal program with typical NDIR and Laser results. . . . . . . . . . . . . 60

6.1 Two-minute average PN concentrations with test run-time for ambient and dry dilution air test groups using Fuel A. . . . . . . . . . . . . . . . . . . . 66

6.2 Relative standard deviations with test run-time for ambient and dry dilution air test groups using Fuel A. . . . . . . . . . . . . . . . . . . . . 67

6.3 Two-minute average particle size distributions for ambient and dry dilution air test groups measured at run end, using Fuel A. . . . . . . . . . . . . . . . . . . . . . 68

6.4 Normalized particle size distributions from Figure 6.3. . . . . . . . . . . . . . . . . . . . 69

6.5 PN concentration during a fuel tank switch (drop in fuel temperature) using Fuel A. . . . 70

6.6 Two-minute average PN concentrations with test run-time for "stock set-up" and both fuel and oil coolers enabled using Fuel A. . . . . . . . . . . . . . . . . . . . . . . . 71

6.7 Images of intake runners (a) before engine cleaning, (b) after treatment procedure, and (c) after manual cleaning procedure. 
6.8 Cylinder 2 injector face images taken (a) after Engine Parameters, (b) after E30, and (c) after T10E10 test groups. . . . . . . . . . . . . . . . . . . 73

6.9 Cylinder 4 intake runners and valves taken (a) after Engine Parameters, (b) after E30, and (c) after T10E10 test groups. . . . . . . . . . . . . . . . . . 74

6.10 PCV filter before use (left) and after E10 test group (right) . . . . . . . . . . . . . . 75

6.11 Two-minute average PN concentration with test run-time for engine parameter tests with

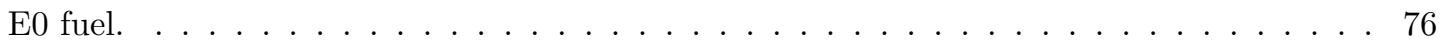

6.12 Two-minute average PN concentrations with mileage accumulation for the two oil changes

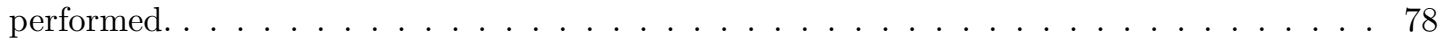

6.13 Two-minute average PN concentrations with test run-time for each test group of the different fuel blends. . . . . . . . . . . . . . . . . . . . 80

6.14 Relative standard deviations with test run-time for each fuel blend test group. . . . . . 81

6.15 Fuel based comparison of PN number concentration size distribution. . . . . . . . . . . 84

6.16 Fuel based comparison of normalised particle size distributions. . . . . . . . . . . . . 84

6.17 Gravimetric filter results grouped by test fuel with overlaid particle number and mass

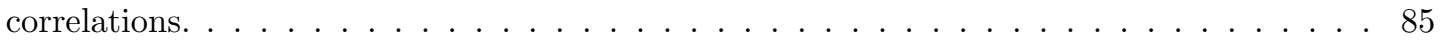

6.18 Elemental-to-organic carbon ratio (EC/OC) for the different fuel blends broken down by

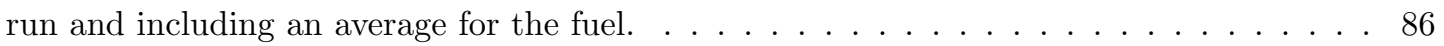

6.19 Fuel average elemental and organic carbon concentrations, with gravimetric concentrations

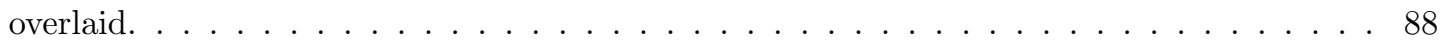

6.20 Average standard emissions measured by the FTIR for the different test fuels. . . . . . . . 90

6.21 Average standard emissions measured by the emissions bench for the different test fuels. . 91

6.22 Average hydrocarbon emissions measured by the FTIR for the different test fuels. . . . . 92

6.23 Average hydrocarbon emissions measured by the FTIR for the different test fuels. . . . . 93

6.24 Two-minute average PN vs. toluene at run end for the different test fuels with LLS

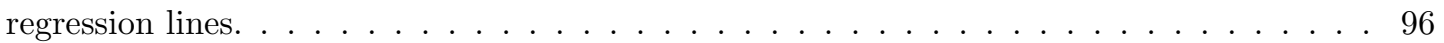

6.25 Two-minute average PN vs. isobutylene at run end for the different test fuels with LLS regression lines. . . . . . . . . . . . . . . . . . . . . . 97

6.26 Average hydrocarbon emissions measured by the FTIR for the two base gasolines (Fuel A and Fuel B). . . . . . . . . . . . . . . . . . . . 100

6.27 Average hydrocarbon emissions measured by the FTIR for the two base gasolines (Fuel A and Fuel B). . . . . . . . . . . . . . . . . . . . . . 101

6.28 PN concentration at run end, averaged for fuel groupings shown, with corresponding PN Index overlaid. . . . . . . . . . . . . . . . . . . . . . . . . . . 102

7.1 Fuel blend comparison of average cold start PN concentration with time. . . . . . . . . . 105

7.2 Average cold start PN concentration (top) and particle size distribution (bottom) with time for E0 fuel. . . . . . . . . . . . . . . . . . . . . . . . 106

7.3 Cumulative normalized concentrations ( $\mathrm{dN} / \mathrm{d} \log \mathrm{dp}$ ), averaged for each test fuel under cold

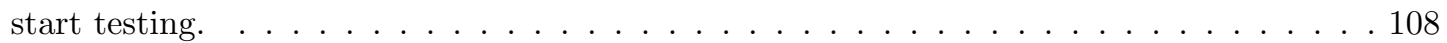

7.4 Fuel blend comparison of hot start PN concentration with time. . . . . . . . . . . . 109

7.5 Hot start PN concentration (top) and particle size distribution (bottom) with time for E0 fuel. . . . . . . . . . . . . . . . . . . . . . . . . . . . 109

7.6 Cumulative normalized concentrations ( $\mathrm{dN} / \mathrm{d} \log \mathrm{dp})$ for each test fuel under hot start testing.110 
7.7 Fuel blend comparison of average cumulative PN concentration during cold and hot starts. 112

7.8 Cold start elemental and organic carbon concentrations. . . . . . . . . . . . . . . 113

7.9 Hot start elemental and organic carbon concentrations. . . . . . . . . . . . . . . . . 114

A.1 Atomic ratios regression for increasing ethanol content. . . . . . . . . . . . . . . . . 134

B.1 Oxygen measurement correction factors. . . . . . . . . . . . . . . . . . . . 139

C.1 Percentage of maximum PN concentration with run time, averaged for each test group. . 143

C.2 Percentage of maximum short-term fuel trim with run time, averaged for each test group. 144

C.3 Comparison of standard emissions as measured by the FTIR, and the emissions bench

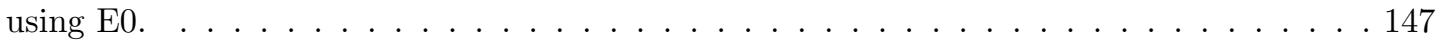

D.1 Average cold start PN concentration (top) and particle size distribution (bottom) with time for E10 fuel. . . . . . . . . . . . . . . . . . . . . . . . 152

D.2 Average cold start PN concentration (top) and particle size distribution (bottom) with time for E30 fuel. . . . . . . . . . . . . . . . . . . . . . . . . . 152

D.3 Average cold start PN concentration (top) and particle size distribution (bottom) with time for E0 fuel (return test group). . . . . . . . . . . . . . . . . . . . 152

D.4 Average cold start PN concentration (top) and particle size distribution (bottom) with time for T10 fuel. . . . . . . . . . . . . . . . . . . . . . . . . . . . . 152

D.5 Single cold start PN concentration (top) and particle size distribution (bottom) with time

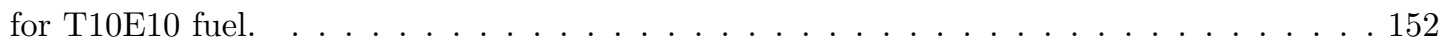

D.6 Average cold start total hydrocarbon (THC) emission for different fuel blends. . . . . . . 153

D.7 Average cold start oxides of nitrogen $\left(\mathrm{NO}_{\mathrm{x}}\right)$ emissions for different fuel blends. . . . . . . 154

D.8 Average cold start carbon monoxide $(\mathrm{CO})$ emissions for different fuel blends. . . . . . . 155

D.9 Average cold start carbon dioxide $\left(\mathrm{CO}_{2}\right)$ emissions for different fuel blends. . . . . . . . 156

D.10 Average cold start oxygen $\left(\mathrm{O}_{2}\right)$ emissions for different fuel blends. . . . . . . . . . . 157

D.11 Hot start PN concentration (top) and particle size distribution (bottom) with time for E10 fuel. . . . . . . . . . . . . . . . . . . . . . . . . 158

D.12 Hot start PN concentration (top) and particle size distribution (bottom) with time for E30 fuel. . . . . . . . . . . . . . . . . . . . . . . . . . . . 158

D.13 Hot start PN concentration (top) and particle size distribution (bottom) with time for T10 fuel. . . . . . . . . . . . . . . . . . . . . . . . . . . . 158

D.14 Hot start PN concentration (top) and particle size distribution (bottom) with time for T10E10 fuel. . . . . . . . . . . . . . . . . . . . . . . . 158

D.15 Hot start total hydrocarbon (THC) emission for different fuel blends. . . . . . . . . . . . 159

D.16 Hot start oxides of nitrogen $\left(\mathrm{NO}_{\mathrm{x}}\right)$ emissions for different fuel blends. . . . . . . . . . . 160

D.17 Hot start carbon monoxide (CO) emissions for different fuel blends. . . . . . . . . . . . 161

D.18 Hot start carbon dioxide $\left(\mathrm{CO}_{2}\right)$ emissions for different fuel blends. . . . . . . . . . . . 162

D.19 Hot start oxygen $\left(\mathrm{O}_{2}\right)$ emissions for different fuel blends. . . . . . . . . . . . . . 163 


\title{
Nomenclature
}

\author{
List of Symbols \\ $\lambda \quad$ Air-Fuel Equivalence Ratio \\ $\phi \quad$ Fuel-Air Equivalence Ratio \\ $\mathrm{r}_{c} \quad$ Compression Ratio
}

$\begin{array}{ll}\text { List of Abbreviations } \\ \text { ACS } & \text { After Cold Start } \\ \text { AFR } & \text { Air Fuel Ratio } \\ \text { BMEP } & \text { Brake Mean Effective Pressure } \\ \text { BTDC } & \text { Before Top Dead Center } \\ \text { CAD } & \text { Crank Angle Degrees } \\ \text { CAFE } & \text { Corporate Average Fuel Economy } \\ \text { CAI } & \text { California Analytical Instruments } \\ \text { CAN } & \text { Controller Area Network } \\ \text { CO } 2 & \text { Carbon Dioxide } \\ \text { CO } & \text { Carbon Dioxide } \\ \text { CoV } & \text { Coefficient of Variance } \\ \text { CVS } & \text { Constant Volume Sampling } \\ \text { DBE } & \text { Double Bond Equivalent } \\ \text { DMA } & \text { Differential Mobility Analyzer } \\ \text { DOHC } & \text { Double overhead camshafts } \\ \text { DR } & \text { Dilution Ratio } \\ \text { DT } & \text { Dilution Temperature } \\ \text { EC } & \end{array}$




\begin{tabular}{|c|c|}
\hline $\mathrm{ECT}$ & Engine Coolant Temperature \\
\hline ECU & Engine Control Unit \\
\hline EEPS & Engine Exhaust Particle Sizer \\
\hline EGR & Exhaust Gas Recirculation \\
\hline EOI & End of Injection \\
\hline ERDL & Engine Research and Development Lab \\
\hline FTIR & Fourier Transform Infrared Spectroscopy \\
\hline GC-MS & Gas Chromatography-Mass Spectrometry \\
\hline GDI & Gasoline Direct Injection \\
\hline HCLD & Heated Chemiluminescence Detection \\
\hline HFID & Heated Flame Ionizing Detection \\
\hline IMEP & Indicated Mean Effective Pressure \\
\hline LLS & Linear Least-Squares \\
\hline LTFT & Long-Term Fuel Trim \\
\hline MBT & Maximum Brake Torque \\
\hline MLR & Multiple Linear Regression \\
\hline MPG & Miles Per Gallon \\
\hline $\mathrm{N}_{2}$ & Molecular Nitrogen \\
\hline NDIR & Non-Dispersive Infrared \\
\hline $\mathrm{NI}$ & National Instruments \\
\hline $\mathrm{NO}_{x}$ & Oxides of Nitrogen \\
\hline $\mathrm{O}_{2}$ & Molecular Oxygen \\
\hline OBD-II & On-board Diagnostic version 2 \\
\hline PAH & Polycyclic Aromatic Hydrocarbons \\
\hline PASS & Photoacoustic Soot Sensor \\
\hline $\mathrm{PCM}$ & Powertrain Control module \\
\hline $\mathrm{PCV}$ & Positive Crankcase Ventilation \\
\hline PFI & Port Fuel Injection \\
\hline PID & Parameter ID (OBD-II) \\
\hline
\end{tabular}


$\mathrm{PM}_{2.5} \quad$ Particulate Matter that is less than $2.5 \mu \mathrm{m}$ in diameter.

PM Particulate Matter

PMP European Union Particle Measurement Programme

PN Particle Number

rpm Revolutions Per Minute

RSD Relative Standard Deviation

RVP Reid Vapour Pressure

SMPS Scaning Mobility Particle Sizer

SOCAAR Southern Ontario Centre for Atmospheric Aerosol Research

SOI Start of Injection

STFT Short-Term Fuel Trim

TDC Top Dead Centre

TEM Transmission Electron Microscopy

THC Total Hydrocarbons

Ti-VCT Twin independent Variable Camshaft Timing

TWC Three Way Catalytic Converter

UBHC Unburned Hydrocarbons

US EPA United States Environmental Protection Agency 


\section{Chapter 1}

\section{Introduction}

Traditional sources of energy are rapidly becoming ecological, physiological, and financial burdens on our world. In addition, the idea of domestic energy security continues to be a defining geopolitical issue as governments strive to reduce their dependence on foreign production of increasingly scarce energy resources. The recent oil price collapse in the fall of 2014 is a prime example of the global financial volatility associated with natural resources, one which has far reaching implications for North American oil production. Nevertheless, the internal combustion engine, which powers nearly all land vehicles, continues to play an essential role in our way of life. As a result, engineers have become increasingly focused on developing clean, alternative energy sources and fuels, as well as improving the combustion processes that make use of them. The automotive industry is but one sector that is undergoing this shift, with increasingly stringent regulations being imposed internationally on fuel composition, vehicle emissions, and fuel economy.

In Canada, the Renewable Fuels Regulations Consolidation of the Canadian Environmental Protection Act came into effect in 2010, stipulating that all gasoline sold nationwide must contain a yearly average of at least $5 \%$ by volume of renewable fuel, such as ethanol [1]. Furthermore, federal emissions regulations outlined by the Canadian Environmental Protection Act provide the emissions regulations for Canadian vehicles and have become more stringent in recent years [2,3]. These fuel and emissions regulations are coupled with an increase in the required fleet-averaged fuel economy minimum to 35.5 miles per gallon (MPG) by 2016 [1] — this is aligned with Corporate Average Fuel Economy (CAFE) regulation in the U.S.

In light of these rapidly changing and tougher regulatory constraints, automotive companies are looking to new technologies in order to meet them. A recent automotive trend has been to use direct fuel injection in gasoline spark-ignited engines to improve their fuel economy and power output. These engines are gaining significant ground in the light-duty engine market, with current projections showing a $50 \%$ market uptake in new vehicles by 2016 [4]. Additionally, several manufacturers have equipped their vehicles with flex-fuel engines that are capable of running up to $85 \%$ by volume ethanol blended

with gasoline (E85). This presents a shift towards the implementation of biofuels in the transportation sector. 


\subsection{Gasoline Direct Injection Overview}

A gasoline direct injection (GDI) engine operates on the same basic principle as all other Otto cycle engines. The difference is that in a GDI engine, the fuel is injected directly into the cylinder, rather than being premixed in the air-induction system as in a carbureted or port fuel injected (PFI) system. Where a modern vehicle equipped with PFI system aims to create an overall stoichiometric and homogeneous fuel-air mixture at all engine speeds and loads, a GDI engine has the ability to produce either a stratified ${ }^{1}$ or homogeneous ${ }^{2}$ charge depending on the time of injection (late vs. early) and injection duration. As a result, both combustion chamber design and fuel injector operation are critical to the success of a GDI fuelling system.

\section{GDI Technology}

There are three main types of injector systems used for GDI engines: spray-guided, wall-guided and air-guided systems - see Figure 1.1 for schematic diagrams. The injector in a spray-guided system fires a select number of fuel plumes towards the spark plug to achieve ignition, while the remaining fuel jets are dispersed into the remaining cylinder volume and are mixed accordingly. A wall-guided engine relies heavily on piston head geometry to mix the injected fuel spray with air and direct it towards the spark plug for ignition. In the air-guided system, the air flow within the cylinder is responsible for directing the fuel charge to the spark plug. First generation GDI engines were largely wall-guided systems, while second generation GDIs are making more use of spray-guided systems; pure air guided systems have rarely been implemented.
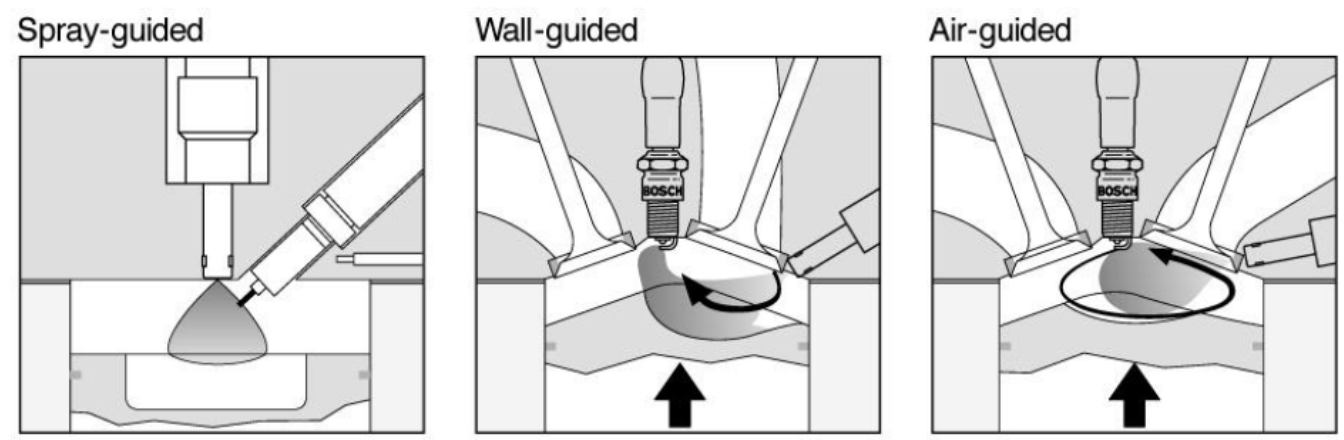

Figure 1.1: Schematic representation of different injection systems in GDI engines [5].

In either case, in-cylinder flows such as swirl, tumble, and squish play critical roles in the fuel-air mixture preparation in GDI engines [5]. Additionally, the two main types of fuel injectors that have been employed in production GDI vehicles are swirl and multi-hole injectors. Swirl injectors impart angular momentum to the liquid fuel via injector geometry, before exiting one or more spray orifices. A multihole injector disperses the liquid fuel spray into several - typically six - plumes of fuel. Historically, swirl injectors have been favoured due to improved atomization of the fuel in the cylinder through increased air entrainment [5]. However, the current prevailing trend is to use high pressure, piezoelectric controlled multi-hole injectors that offer superior injector control. A study by Price et al. [6] found that an engine with a spray-guided system and multi-hole injector combination emitted particulate matter (PM) an

${ }_{1}^{1}$ Stratified operation-injection occurs late during the compression stroke.

${ }^{2}$ Homogeneous operation-injection occurs during the intake stroke. 
order of magnitude less than an engine with a wall-guided and swirl injector system. This was due in large part to the superior mixture preparation that the high pressure spray-guided system provides. Differences also appear in the size of particles emitted from both systems; Zhang and McMahon [7] show wall-guided systems to produce larger nano-particles in what is known as the "accumulation mode", while spray-guided systems produce a more bimodal distribution during standardized testing ${ }^{3}$.

\section{Benefits and Shortcomings of GDI}

GDI engines provide many operational benefits over traditional port fuel injection (PFI) engines. According to Zhao et al. [5], a GDI system offers improved fuel economy over a PFI system through the reduction of pumping and heat loses, as well as from increased volumetric efficiency resulting from charge cooling and high compression ratios. Further benefits include finer air-fuel ratio (AFR) control, improved transient engine response, increased exhaust gas recirculation (EGR) tolerance limit, and reduced cold-start unburned hydrocarbon (UBHC) emissions [5]. However, inherent issues related to GDI engines include elevated UBHC emissions at light loads, elevated oxides of nitrogen $\left(\mathrm{NO}_{\mathrm{x}}\right)$ emissions at high loads, greater PM emissions, three-way catalysis utilization issues, and stratified charge combustion control complexities [5].

\subsection{GDI and Emissions}

Injecting the fuel directly into the cylinder brings about many challenges related to mixture preparation. These challenges are manifested as increased gaseous and PM emissions, as compared to a similar PFI engine, as their mechanisms are changed or exacerbated. The following will discuss some of the emissions issues related to GDI engines; a more comprehensive discussion of GDI pollutant formation mechanisms is provided in Chapter 2.

The major constituents of exhaust emissions from spark-ignited engines have long been known to consist of UBHCs, $\mathrm{NO}_{\mathrm{x}}$, and carbon monoxide (CO). In all spark-ignited engines, UBHC emissions are formed from four possible mechanisms: (1) unburned fuel escaping combustion from flame quenching, (2) the filling of in-cylinder crevices with un-burned fuel-air mixture that escapes combustion, (3) absorption and desorption of fuel vapour into lubrication oil on cylinder walls, and (4) incomplete combustion [8]. GDI engines suffer from additional mechanisms related to poor fuel atomization and vaporization. Fuel wall impingement causes liquid droplets of fuel to form, which do not readily burn in the combustion process [5]. The sac volume in the injector is another such source of liquid fuel escaping the atomization and vaporization process [5]. The possibility of running overall lean during stratified charge operation may also contribute to UBHC emissions, due to flame propagation issues from rich zones near the spark gap to much leaner zones along the periphery of the cylinder [5]. $\mathrm{NO}_{\mathrm{x}}$ formation and emissions are highly dependent on combustion temperature, as well as the abundance of oxygen in the burned exhaust gases [8]. At high loads where the temperature of combustion is high, formation of $\mathrm{NO}_{\mathrm{x}}$ in a GDI engine is augmented during stratified operation; excess oxygen in the cylinder reacts with the atmospheric nitrogen that is not directly involved in the combustion process [5].

PM from a GDI engine is thought to form much in the same way as typical diesel engines; from un-vaporized liquid fuel droplets and from locally rich fuel-air mixtures in the gaseous phase $[5,8]$. In GDI engines, locally rich fuel cores are readily formed if charge mixing is insufficient. Furthermore,

${ }^{3}$ Standard drive cycles for emissions testing include US EPA FTP-75 and US06, as well as European NEDC. 
liquid fuel droplets from piston and cylinder wall wetting, as well as injector sac volumes are inherent to direct injection engines. These pools of liquid fuel that collect on piston surfaces emit PM through diffusion burning in the combustion process [9].

Current emissions control systems do a good job of reducing vehicle produced pollutants. The introduction of catalytic converters in the mid-1970s, as well as the implementation of EGR and evaporative emissions control systems, has reduced the emission of these regulated compounds from vehicles thereby, mitigating their ecological and health impacts. Three-way-catalytic converters (TWC) are universally equipped on modern gasoline vehicles and are very effective at reducing $\mathrm{NO}_{\mathrm{x}}$, and oxidizing the $\mathrm{CO}$ and UBHC constituents of engine exhaust gases. However, since PM is not typically produced in significant quantities by PFI engines, PM emission control systems have not been thoroughly developed for gasoline engines. PM capture techniques, such as diesel particulate filters (DPF), have proven useful in diesel applications and some recent studies have also seen useful application in GDI engines [10]. However, their necessity to regenerate - i.e. burn off deposited particles from the filter surface to reduce back-pressure on the engine exhaust - can increase fuel consumption, making it potentially unattractive to consumers and a fuel economy requirement burden.

\subsection{Health Effects of Particulate Matter}

The harmful health effects of internal combustion engine exhaust are well documented in the healthcare field. While regulated gaseous components of the exhaust stream $\left(\mathrm{CO}, \mathrm{NO}_{\mathrm{x}}\right.$, and $\left.\mathrm{UBHC}\right)$ can be effectively scrubbed by a TWC, PM emissions remain an issue for GDI engines. Several recent studies have concluded that even moderate-to-low level exposure to PM has profound negative health impacts including higher incidences of cardiovascular diseases and cancer $[11,12,13,14]$. Short-term exposure to $\mathrm{PM}$ is linked to altered cardiac autonomic function, arterial vasoconstriction, and increased hospital admission for cardiovascular problems, strokes, and mortality. Furthermore, long-term exposure to PM is associated with, among other things, increased cardiovascular mortality, inflammatory lung injury, and subclinical atherosclerosis [11]. The American Heart Association notes that the elderly, those with diabetes, and those with pre-existing coronary artery disease, are at an increased risk for death and non-lethal health events related to cardiovascular diseases from exposure to PM [13].

Pope et al. [12] analyzed the influence of changes in PM pollution levels to changes in life-expectancy from 1980-2000 in the 51 largest metropolitan areas in the United States. When adjusted for socioeconomic, demographic, and smoking prevalence factors, the data showed a correlation between reductions in PM pollution levels and an increased life-expectancy of a few months [12]. On average, reductions in $\mathrm{PM}_{2.5}$ (PM that is less than $2.5 \mu \mathrm{m}$ in diameter) pollution levels led to an increase of life-expectancy by 0.4 years; this value grew to 0.82 years in areas where reductions in PM were double the average of $6.52 \mu \mathrm{g} / \mathrm{m}^{3}$. Figure 1.2 provides a size scale of $\mathrm{PM}_{2.5}$ to other common airborne material.

The International Agency for Research on Cancer - under the auspices of the World Health Organization - confirmed the carcinogenicity of whole diesel engine exhaust by raising it to a Class 1 carcinogen [14]. The same report also raised gasoline engine exhaust to a Class $2 \mathrm{~A}$ carcinogen, meaning it is possibly carcinogenic to humans. The research showed that extracted particles from diesel exhaust caused benign and malignant lung tumours in rats. Extracted organic condensates from gasoline exhaust also showed increased lung carcinomas in mice and rats. In both cases, extracted particles caused DNA damage to mammalian cell cultures. 


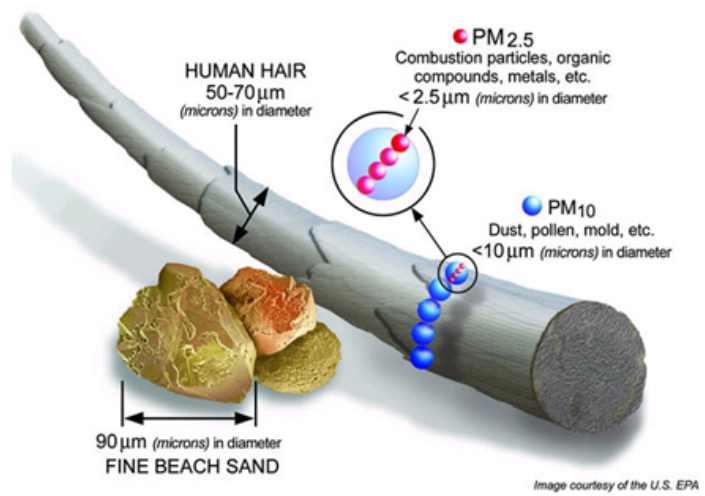

Figure 1.2: PM size comparison (courtesy of the US EPA).

\subsection{PM Regulations Driving Research}

It is evident that the inhalation of PM emitted from combustion processes can be highly detrimental to human health. As a result, stricter regulations on the emission of these carcinogenic particles is becoming more widespread. European regulations have seen a significant tightening of allowable PM emissions in recent years. The Euro 6 regulation currently limits the emission of PM from light duty gasoline engines to $4.5 \mathrm{mg} / \mathrm{km}$ and $6 \times 10^{12} \# / \mathrm{km}$ [15]. In the US, the upcoming adoption of the CARB ${ }^{4} \mathrm{LEV}$ III and US EPA $^{5}$ Tier III emissions standards in 2017 will see the PM emission standard drop to $3 \mathrm{mg} / \mathrm{mi}$ [15]. Canada, by extension of geographical proximity, will also be subject to the US emissions regulations.

Therefore, research into mitigating PM emissions from GDI engines is not only important for protecting human health, but also a necessity for automotive manufacturers to ensure continued certification of their vehicles. These external forces have driven GDI research and development to the forefront of recent engine advancement.

${ }^{4}$ CARB - California Air Resources Board

${ }^{5}$ US EPA - United States Environmental Protection Agency 


\section{Chapter 2}

\section{Emissions from GDI Engines}

The following chapter serves as an introductory literature survey of recent research conducted on GDI engines, with particular emphasis on PM emissions. A summary of PM formation and morphology mechanisms is first provided, followed by an analysis of injector spray characteristics. The influence of engine parameters as they pertain to PM emissions and other key combustion attributes are also presented, as well as a discussion of fuel composition and PM emissions. The effect of catalytic converters and gaseous emissions from GDI engines is also considered. The chapter concludes with the topic of PM sampling techniques and considerations.

\subsection{PM Formation and GDI PM Morphology}

An understanding of the formation mechanisms and morphology of PM is critical to this study. As previously discussed, PM from a GDI engine is formed through similar mechanisms as those in a diesel engine. In both instances, diffusion burning of fuel rich cores and liquid fuel droplets are the major contributors to particle emissions. According to Heywood [8], PM formation begins with a particle nucleation mechanism, followed by in-cylinder surface growth and agglomeration, and culminates with adsorption and condensation downstream of the cylinder; see Figure 2.1 for a diagram of PM formation mechanisms.

Nuclei particles are formed when fuel molecules are decomposed to unsaturated hydrocarbons and recombined as polyacetylenes and polycyclic aromatic hydrocarbons (PAH), which condense to form small nano-scale particles (particle diameter $<2 \mathrm{~nm}$ ) [8]. Particles then increase in size by surface growth, coagulation, and aggregation mechanisms, before escaping the cylinder [8]. As the exhaust cools, adsorption and condensation of lighter UBHCs in the exhaust stream further modify the particle sizes and counts [8]. Oxidation of particles into gaseous products may occur at any stage of the PM formation process; the net PM emissions represent an equilibrium between the oxidation and formation mechanisms. Particle size distributions are typically bimodal in nature, with a "nucleation mode" occurring in the 5-20 nm range and an "accumulation mode" in the 70-100 nm range. Under certain conditions, monomodal or even trimodal distributions are measured.

Several recent studies have investigated the morphology of PM derived from a GDI engine $[16,17,18]$. Barone et al. [16] studied the particle morphology from a production engine under two injection strategies; 


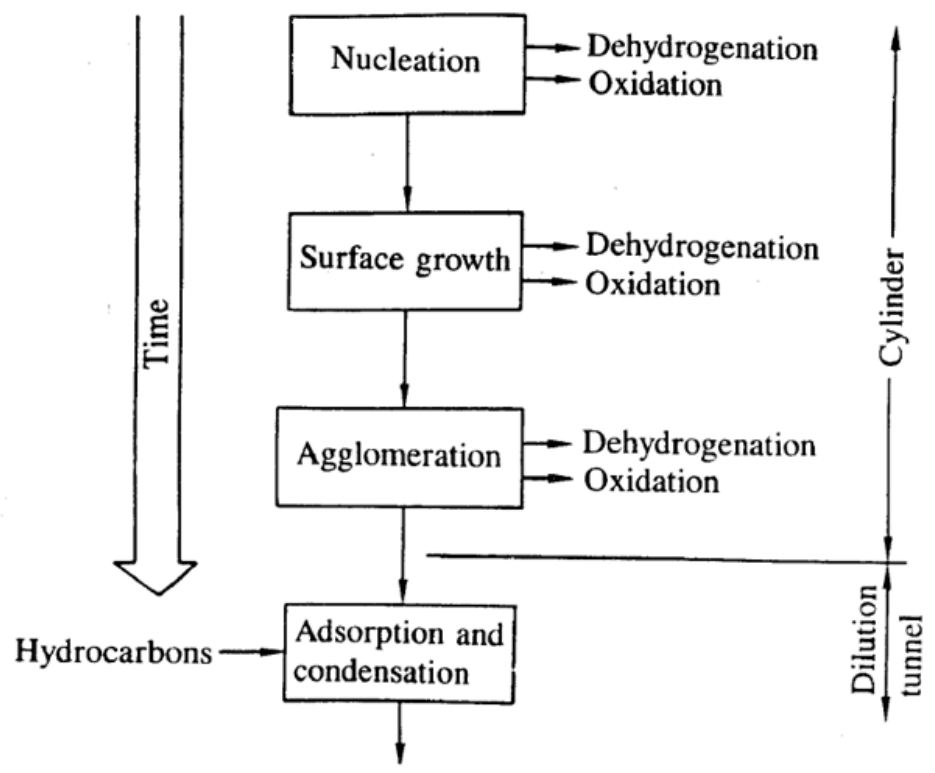

Figure 2.1: PM formation mechanisms [8].

an early injection strategy $\left(320^{\circ} \mathrm{BTDC}^{1}\right)$ and a strategy optimized for low particle emission $\left(280{ }^{\circ} \mathrm{BTDC}\right)$. Through transmission electron microscopy (TEM) analysis, particles from the early timing were observed to consist of solid spheres under $25 \mathrm{~nm}$ in size, liquid droplets, and nanoparticle aggregates; only solid spheres and nanoparticle aggregates were observed in large numbers for the low particle injection timing regime. The primary particles of the aggregates under the low particle injection timing had a smaller modal average $(10-15 \mathrm{~nm})$ but a larger range of sizes $(7-60 \mathrm{~nm})$ compared to the early injection timing case. This was explained by the fact that on the soot particle size scale - the scale at which primary particles collide - fuel-air mixing is relatively homogeneous, whereas on the scale of the engine cylinder, fuel-air mixing is heterogeneous [16].

Sgro et al. [17] investigated nuclei (non-volatile core) particle formation and growth from a production GDI engine by analysing where the particles were formed; in the combustion chamber, in the exhaust system, or in the sampling system. This was done by comparing measured charge fraction distributions of the particles to those calculated based on temperature; high temperature for in-cylinder particles and lower temperature for cylinder-out particles. Results showed that core particles were ionized in the combustion chamber and that heterogeneous condensation of particles occurred at lower temperatures. These results confirm the PM formation mechanisms discussed above in [8].

A study performed by Maricq [18] sought to capture the soot chemistry of diffusion flames as an analogue to locally rich combustion conditions associated with typical GDI engines. Particle sampling performed at different flame heights showed that nucleation mode dominated particles developed low in the flames before progressing through a bimodal phase and on to a final accumulation mode of agglomerated soot at elevated flame heights. This observation highlights the temperature dependence of PM formation mechanisms discussed by Heywood [8]. TEM analysis yielded further observations; gasoline particles sampled low in the flame were largely spherical in nature and approximately $50 \mathrm{~nm}$

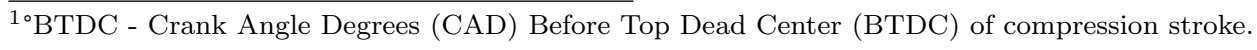


in diameter, while at a higher flame heights, the particles demonstrated agglomerated morphology more typical to soot [18]. Soot particles from the gasoline flame were shown to be more compact than particles from the E85 (ethanol-gasoline blend with $85 \%$ by volume ethanol) blend. Furthermore, the mean diameter of the primary particles from gasoline were twice the diameter of the primary particles generated by the E85 blend. Maricq reasons that while this difference in soot agglomerates can be explained by the condensation of semi-volatiles during sampling, it is likely that particle growth occurs in the flame itself [18].

\subsection{GDI Spray Characteristics}

The injectors in a GDI engine play a critical role in combustion characteristics and pollutant formation. As a result, much research into GDI injectors and spray effects has been conducted recently. Optical studies, such as $[6,9,19,20]$, have provided much insight into fuel spray interactions within the cylinder, and have helped to explain many observed combustion and pollution phenomena.

In an optical study, Drake et al. [9] investigated the presence of fuel films on the piston top of a wall-guided GDI engine using a swirl type injector and a multi-hole type injector. Through endoscopic image analysis, the authors found that the multi-hole injector provided a more disperse spray pattern than the swirl type. Furthermore, late combustion luminosity, as evidence of pool fires, was notably absent with the multi-hole injector. Wall-film measurements showed an order of magnitude thinner film heights and smaller volumes, and to a lesser degree, smaller film areas for the multi-hole injector [9].

Price et al. [6] studied the influence of injection system type on PM and UBHC emissions; wall-guided with swirl type injector vs. spray-guided with multi-hole injector. In general, Price et al. conclude that the spray-guided system emitted PM mass concentrations an order of magnitude less than the wallguided system because they provided superior mixture preparation. They note that the spray-guided system is more effective at vaporizing fuel, produces less wall wetting, and emits very few accumulation mode particles as evidence of superior mixing performance.

Serras-Pereira et al. [19] compared a single injection strategy to a triple injection strategy on the basis of piston and cylinder wall wetting. The triple-injection strategy showed strong reductions in fuel impingement levels, especially at a cold engine coolant temperature (ECT) of $20{ }^{\circ} \mathrm{C}$. In a similar light, Warey et al. [21] directly simulated the effect of piston wall wetting by injecting liquid fuel onto the piston crown via an injector probe in a nominally PFI engine. PM emissions were shown to have a strong response to piston wetting, with both particle number $(\mathrm{PN})$ concentrations and mass measurements falling to baseline levels when the injector probe was shut off.

Both Serras-Pereira et al. [19] and Oh et al. [20] also found that spray characteristics varied with respect to fuel composition. Under lean stratified operation, Oh et al. [20] saw lower fuel concentration variation of the spray when ethanol was injected instead of isooctane. Additionally, the area of the dispersed spray was greater for the ethanol injection, which according to the authors, implied a faster diffusion velocity of the fuel yielding enhanced fuel-air mixing [20]. Serras-Pereira et al. [19] also observed a temperature-fuel composition dependency for the spray characteristics in their study. At an ECT of $20{ }^{\circ} \mathrm{C}$ both gasoline and isooctane produced very similar spray formations and spray developments; however, only gasoline experienced spray collapse - when plumes are drawn towards the centre of the combustion chamber - in some of its plumes at an ECT of $90{ }^{\circ} \mathrm{C}$. Further, initial penetration for the gasoline plumes were shown to reduce in the heated condition and were observed to widen and break 
apart more quickly than at $20{ }^{\circ} \mathrm{C}$ - demonstrating that the temperature effect on isooctane was much more subdued. Measured impingement levels corroborated these optical observations. At stoichiometric and homogeneous operation, Chen et al. [22] observed a detrimental effect of increasing ethanol content and decreasing temperature on injection spray characteristics, including reduced spray dispersion leading to increased mixture heterogeneity. This disagreement with the findings of $\mathrm{Oh}$ et al. [20] can be explained by the effect of ECT on spray characteristics; Oh et al.'s findings were produced at an ECT of $80{ }^{\circ} \mathrm{C}$, mitigating the issue of the high heat of vaporization of ethanol. In terms of spray development, injection plumes were more defined and dispersed less under cold operation than under warm operation for all fuels tested; increasing ethanol content further increased both spray duration and area [22].

\subsection{GDI Engine Parameters and PM}

As shown above, engine operating characteristics have significant influence on the spray development in GDI engines. PM emissions are therefore closely related to several key engine parameters, such as ECT, spark timing, injection timing and duration, fuel-air equivalence ratio, engine load, and valve timing. Many studies have directly investigated the sensitivity of altering these parameters on PM emissions. The following will explore the results of those studies on the basis of the individual parameters.

Figure 2.2 presents compiled PM emission data from some of the sources reviewed herein, categorized by some of the topics discussed. In general, PM emissions increased with increasing engine load, increasing engine speed, and increasing injection timing retard. PM emissions typically decreased with increasing ECT, decreasing engine speed, and to some extent, increasing ethanol content - the effect of fuel is discussed in a subsequent section.

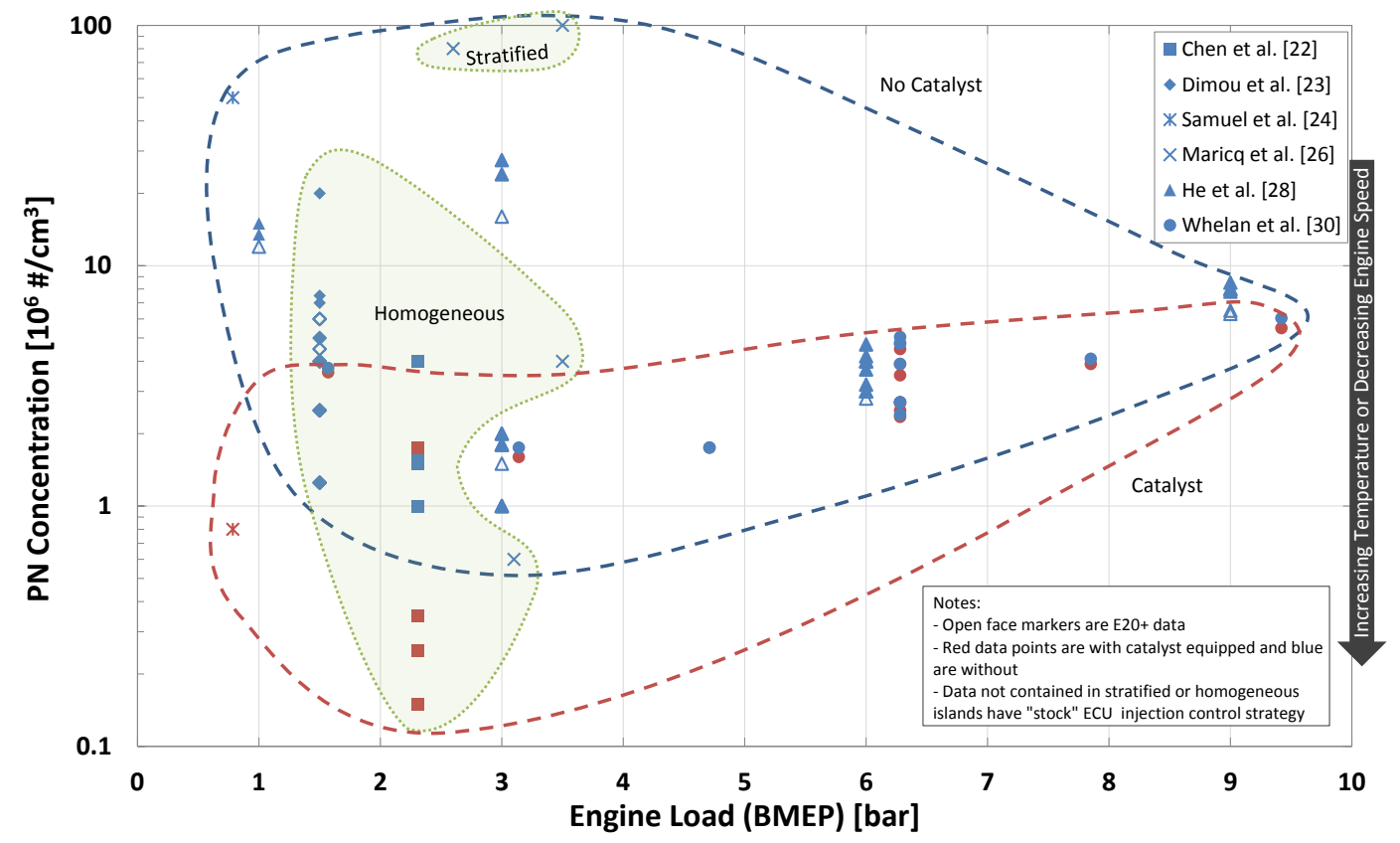

Figure 2.2: Collected PN concentrations with engine load data from some reviewed sources. See notes on figure for further clarifications. 


\subsubsection{Engine Coolant Temperature}

The discussion on GDI spray effects in the previous section introduced the importance of in-cylinder temperatures on the development of the injected fuel sprays, and the potential for them to cause or exacerbate the aforementioned PM formation mechanisms. Accordingly, the effect of ECT on PM emissions has been studied extensively in recent investigations.

Chen et al. [22, 23] looked at this effect on engine out emissions from a production engine under homogeneous charge operation. Both studies found that increasing ECT from $20{ }^{\circ} \mathrm{C}$ to $80-90{ }^{\circ} \mathrm{C}$ clearly decreased PM emissions because of the higher temperature aiding fuel spray vaporization. In addition, the warm operation point produced lower coefficients of variance $(\mathrm{CoV})$ values for calculated indicated mean effective pressures $\left(\mathrm{IMEP}^{2}\right)$ than cold operation for all fuels, indicating a strong temperature dependence on combustion stability for GDI engines [22].

Dimou et al. [24] also sampled engine out emissions from a production engine at a wide range of cold $\operatorname{ECTs}\left(0,7,15,20,30\right.$, and $\left.40{ }^{\circ} \mathrm{C}\right)$. They found the following: (1) above $200 \mathrm{~nm}$ particle diameter, no temperature dependence was seen for PN concentrations; (2) below $100 \mathrm{~nm}$, a significant and nonlinear rise in $\mathrm{PN}$ concentration emissions was observed below $15{ }^{\circ} \mathrm{C}$; and (3) a moderate and linear rise was observed from 40 to $20{ }^{\circ} \mathrm{C}$. Once again, fuel vaporization issues causing fuel-wall impingement was hypothesized as the source of this observation [24]. Samuel et al. [25] detail a similar temperature and PM emission trend in a study conducted for cold-start, warm-up conditions in a production GDI engine. Particles in the 5-25 nm range represented $99 \%$ of the total PM emissions over the entire test. Approximately two minutes into the test, the number of particles greater than $25 \mathrm{~nm}$ was observed to have dropped significantly, and by the fourth minute they were observed to have decreased by two orders of magnitude from their initial concentrations [25]. Furthermore, total particle emissions were noted to be an order of magnitude less during warm-up idle period following a cold start, than during the cold start itself.

The above investigations mimic the results produced from GDI vehicles over standard emissions tests, such as the FTP-75 or US06 drive cycles. Both Chan et al. [10] and Khalek et al. [26] saw greater PM mass emissions under Phase 1 (cold start) of the FTP-75 test cycle than under Phases 2 or 3 . Based on the above sources of information, the influence of ECT on PM emissions can be said to be well understood. Higher ECTs provide for warmer in-cylinder temperatures mitigating the presence of liquid fuel and aiding the fuel vaporization and mixture process. Looking at Figure 2.2, this result is indeed shown by the general trend of decreasing PN concentrations with increasing ECT.

\subsubsection{Ignition Timing}

The ignition timing of the fuel-air mixture is significant for any spark-ignited engine, as it dictates the timing and magnitude of the in-cylinder peak pressure and temperature. In GDI engines, ignition timing also dictates the amount of time available for mixture preparation as the time between the end of injection (EOI) timing and the spark event.

Maricq et al. [27] investigated the influence of spark timing on PM emission from a production GDI engine. Under both stratified and homogeneous charge regimes, PN counts were shown to increase with spark timing advance ${ }^{3}$, with stratified operation resulting in 25 times higher PM emissions than

${ }^{2}$ IMEP is an indication of the engine loading.

${ }^{3}$ Timing can be advanced (earlier) or retarded (later) from the optimum timing, known as timing for maximum brake torque (MBT). 
homogeneous operation. Particle distributions for both instances showed slight shifts to larger particles at earlier spark times. The authors reason that a greater number of larger particles appear with advancing spark timing because there is less time available for fuel volatilization between EOI and the spark event. In a more recent study by Price et al. [6], advancing spark timing under homogeneous and stoichiometric operation also increased the PM emissions, again demonstrating the temporal dependence of fuel vaporization caused by ignition and EOI timing in relation to each another.

A later study by Price et al. [28] once again found that PN concentrations generally increased with advancing ignition timing. According to the authors of this study, this result was to be expected as the temperature in the expansion and exhaust strokes would be lower with earlier ignition resulting in a decrease in post-flame oxidation of organic compounds. This oxidation would result in a reduction in the number and sizes of the emitted PM as discussed in [8] and shown in Figure 2.1.

\subsubsection{Injection Timing and Duration}

The fuel injection operation controls many aspects of the combustion process in a GDI engine. Parameters such as injection timing and duration directly affect PM formation through mixture preparation and wall impingement processes [5]. Much of the research concerning PM emission from GDI engines has therefore been concerned with the act of fuel injection. Figure 2.2 shows the effect of injection timing on PM emissions with the highlighted stratified and homogeneous islands. Based on this figure, it can be said that stratified operation promotes the emission of PM.

Maricq et al. [27] explored the effect of EOI on PM emissions by sweeping it at constant engine speed and overall lean AFRs. Compared to homogeneous operation, stratified injection resulted in both PN and mass emission increases by a factor of 20. Closer inspection of the stratified regime showed that retarding EOI timing also resulted in a distribution shift towards smaller particles, followed by a shift to larger ones. Maricq et al. [27] explained this observation to be the result of the increasing number of locally rich regions - responsible for PM formation - in the stratified charge as EOI is retarded. In homogeneous operation, the particle mass emissions remained constant with advancing EOI timing; however, total PN emissions appeared to decrease over the same interval [27]. This was attributed to a shifting particle distribution, with mean particle sizes increasing with advanced EOI timing [27]. He et al. [29] also found a strong dependence of PM emissions on injection timing, where injection retard from top dead centre (TDC) of compression caused a reduction in PN concentrations.

The study by Price et al. [28] also looked at injection timing with respect to PM emissions under homogeneous charge operation. In this case, start of injection (SOI) timing was varied and PM emissions were shown to increase when injection timing was retarded. Further investigation showed that PM emissions as a function of SOI are dependent on two important factors: time for mixture preparation and piston and wall wetting. A previously mentioned study also found that even though the PM size distributions maintained their shape, retarding injection timing increased PN concentrations by an order of magnitude through the injection timing sweep [6]. Comparing the two injection systems (wall-guided vs. spray-guided), the spray-guided system gave a monotonic increase of PM emissions with EOI retard, while the wall-guided system gave a parabolic relation [6]. This was observed because of the competing PM formation effects of inadequate mixture preparation and wall wetting present in a wall-guided system.

Drake et al. [9] showed that earlier and later injection timings from the optimal point gave rapid drop offs in combustion performance due to partial burns and random misfires. From the optical data presented, retarding EOI under stratified operation caused the wall-film volume to increase for 
both injectors studied (swirl and multi-hole). The smoke number - an unsophisticated measure of PM emissions - increased with EOI retardation, and was linearly correlated to the wall-film mass. This compares favourably with results of [21] as stated in the previous spray discussion. A different study [20] showed that combustion efficiency first increased with EOI timing retard, reaching a maxima before it proceeded to decrease. It was suggested that this observation was likely due to the effect of the ambient pressure in the cylinder when the injection begins, with too high a pressure (late EOI) causing spray stagnation (poor mixing) and too low a pressure (early EOI) causing failed stratification of the charge for ignition. Additionally, IMEP was observed to increase with EOI retardation.

\subsubsection{Equivalence Ratio}

Local fuel-air equivalence ${ }^{4}$ ratios $(\phi)$ within the cylinder are known to have a strong effect on observed particle emissions, with locally rich regions contributing significantly to PM formation in GDI engines [5]. To complicate matters, even though the engine may be operating overall lean, a high degree of stratification can still induce many regions of locally rich AFR. Therefore, it is important consider both the commanded $\phi$ and the injection strategy (stratified vs. homogeneous) when analyzing the effect of $\phi$ on PM emissions for GDI engines.

Some studies have looked at $\phi$ or AFR in terms of PM emissions directly. Under homogeneous operation, Price et al. [28] found that decreasing $\lambda$ (enriching $^{5}$ ) lead to an increase in PN concentrations regardless of the fuel used, though a fuel dependency was noted in the PM emission response to enriching. In another study, Price et al. [6] swept AFR from lean to rich under homogeneous operation and found that PM formation was inversely proportional to $\lambda$, or directly proportional to $\phi$. Enriching from $\lambda=1.2$ to $\lambda=1.1$ doubled the opacity of the exhaust emitted from a production engine in [17] —opacity is used as a less sophisticated measure of PM emissions. Sakai et al. [30] also examined the role of equivalence ratio on PM emissions by sweeping $\phi$ at constant engine operation. They found that even minor changes in $\phi$ caused large changes in total concentrations, along with a size distribution shift from smaller particles to larger ones with increasing $\phi$.

Although the influence of $\phi$ is clearly evident, the fact that most new vehicles are equipped with a TWC implies that they must run in closed loop $\phi$ control $^{6}$ to properly operate it. This means that even though it may be advantageous to run overall lean from a fuel economy point of view, it may negate the operation of current gaseous emissions control strategies. Furthermore, the use of a TWC does provide some reductions in PM emissions, as will be discussed in Section 2.5.

\subsubsection{Engine Operating Condition}

The speed and loading of an engine have important interactions with both in-cylinder temperatures and mixture preparation. A faster moving piston results in less time for mixture preparation and can induce piston wall wetting effects, while a slower engine reduces in-cylinder flows that promote mixing. By the same token, a more heavily loaded engine will increase in-cylinder temperature and alter the rate of fuel vaporization and as a result, reduce the amount of liquid fuel in the charge; however, a more loaded engine requires a greater mass of fuel provided by longer injection durations. Figure 2.2 provides a

${ }^{4} \phi$ is a representation of the fuel-air proportions; stoichiometric AFR over the actual AFR for the specific fuel. Values greater than unity represent fuel-rich conditions and values less than unity are fuel-lean.

${ }^{5} \lambda$ is the inverse of $\phi$.

${ }^{6}$ Closed loop control for TWC involves setting the commanded $\phi$ to stoichiometric $(\phi=1)$, and cycle between slightly lean $(\phi<1)$ and slightly rich $(\phi>1)$ to operate the catalyst 
compilation of various sources on PM emission levels with varying loads and engine speed sweeps. From this figure, it is generally noted that increasing engine speed correlates with increasing PN concentration emissions, while the loading effect is more parabolic. However, what is also evident here is that the effect of high engine speed and load on PM emissions has not thoroughly been reviewed.

Maricq et al. [27] found that PM emissions increased with increasing load and increasing engine speed for both stratified and homogeneous operation. The explanation provided for this observation is that larger loads require greater fuel mass, resulting in charge cooling and reduction in rate of fuel volatilization. Additionally, increasing engine speed results in a reduction in available time for vaporization, causing an increase in PM emissions. Whelan et al. [31] specifically investigated the effect of engine speed and load on PM emissions from a production GDI engine with a wall-guided injection system. Engine load was swept from 20 to $120 \mathrm{Nm}$ by $20 \mathrm{Nm}$ increments over an engine speed range of 1600-3600 rpm. At a constant engine speed, the PN concentrations displayed a parabolic-like relation with engine loading - emissions first decreased to a minima before rising again, as the engine load was increased. The relation of PM emissions with engine speed at constant loading was more complex; at $80 \mathrm{Nm}$, for example, PM emissions first declined sharply as engine speed increased from 1600 to $2200 \mathrm{rpm}$, before increasing quickly again to $3000 \mathrm{rpm}$ and levelling off at $3600 \mathrm{rpm}$ [31]. In this case the ECU, which controls many of the engine parameters discussed in this section, regulated fuel injection pressure and timing as per the manufacturer's original specifications, so conclusions independent of these important characteristics were not made.

On a production engine with a stock ECU calibration, Wallner and Miers [32] found a fuel dependency with engine load on combustion stability; above $100 \mathrm{Nm}$ a large disparity was seen between the tested ethanol-gasoline blends. The authors explain that because the engine is equipped with a knock sensor, which retards spark timing to avoid detonation, the fuels with higher ethanol content required less ignition retard at higher loads due to their increased octane rating. Though, in a different study by He et al. [29], total PN concentrations were significantly higher at low engine speeds $(<1500 \mathrm{rpm})$ and loads than at higher operating points, regardless of fuel composition. Here the authors explain this to be the result of the low and unstable fuel rail pressures present at slow engine speeds - the cam-driven high pressure fuel pump caused pressure oscillations across the fuel rail at low engine speeds [29]

Results from investigations that studied GDI vehicles over standard emissions tests, such as the FTP-75 or US06 drive cycles, also showed a dependence of PM emissions on engine speed and load. Both Khalek et al. [26] and Chan et al. [10] saw greater PM mass emissions under the US06 drive cycle than under the FTP-75 drive cycle; the US06 represents a more rigorous drive cycle, causing the engine to undergo higher loading conditions. Khalek et al. [26] also found that PN emissions were 100 to 1000 times greater during and immediately following an acceleration event. This observation further highlights the importance of transient manoeuvres on PM emission levels.

\subsubsection{Valve Timing}

Very few studies have been performed on valve timing optimization for PM emissions. This area of discussion is important for modern engines, as valve timing control is now fairly ubiquitous on modern GDI vehicles, which could present an easy to implement control strategy.

The effect of valve timing - specifically variable valve timing - was explicitly studied by Chen et al. [23] on a homogeneous, spray-guided production GDI engine at constant speed and load conditions. Increasing valve overlap lead to a decrease in PM emissions, both in number and mass concentrations. 
This was explained to be a result of high internal EGR with increasing valve overlap, which creates a higher in-cylinder gas temperature during compression. This aids in the vaporization of the liquid fuel, thereby increasing the homogeneity of the fuel-air charge mixture and mitigating PM formation. This is in addition to the $\mathrm{NO}_{\mathrm{x}}$ control that running internal EGR provides, through the reduction in peak cylinder temperatures.

\section{$2.4 \quad$ Fuel Effects and PM}

Fuel composition is also critical to the formation of PM in GDI engines and many of the previously mentioned studies have explored the effect of fuel chemistry on PM emissions in conjunction with other factors. Studies like [10, 22, 23, 24, 28, 29] have directly assessed the effect of alcohol content in gasoline fuel on PM emissions, while others have also isolated for aromatic and paraffin hydrocarbon compounds typically found in large quantities in commercial gasoline. Physical properties such as volatility have also been researched [26]. The following will discuss some of the results of those studies as they pertain to PM emissions; gaseous emissions are explored in a subsequent section. Gasoline-alcohol blends are expressed here in a percent volume of alcohol basis; i.e. a $10 \%$ by volume ethanol- $90 \%$ by volume gasoline blend is expressed as E10.

Figure 2.3 represents lines of constant ethanol content on a plot comparing PN concentrations to engine load, based on several of the sources discussed below. Please note that the PN concentration scale is truncated compared to the one in Figure 2.2. In some instances, increasing ethanol gave a notable decrease in PM emissions; however, in other studies this was not so clear as evidenced by disorder of lines of constant ethanol in Figure 2.3.

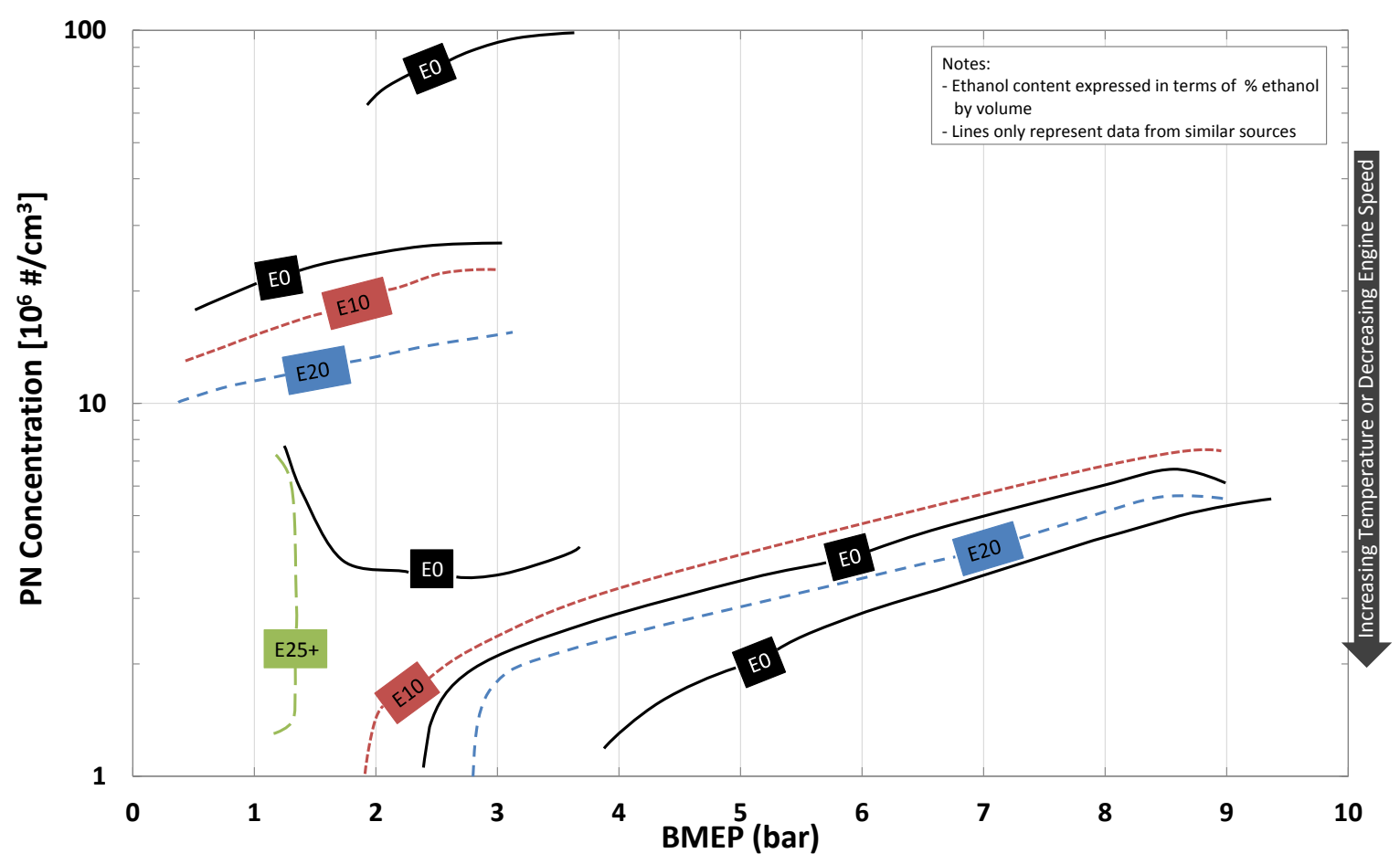

Figure 2.3: Lines of constant ethanol content for PN concentrations vs. engine load. See notes on figure for further clarifications. 
Price et al. [28] found that ethanol blends (E85 and E30) showed the lowest PM emissions, followed by methanol blends (M85 and M30) for lean fuel-air mixtures; however, pure certification gasoline produced the lowest PM emissions for rich mixtures. Chen et al. [23] observed a reduction of PM emissions when running E10 over E0 (gasoline) with a cold ECT of $20^{\circ} \mathrm{C}$. The availability of oxygen atoms in the fuel from the ethanol is believed by the authors to have aided the oxidation of local fuel rich pockets in the charge. The converse was true for a hot ECT of $90{ }^{\circ} \mathrm{C}$, owing to the higher enthalpy of vaporization of ethanol giving a more inhomogeneous charge. Using a single-cylinder research variant of the same engine in [23], Chen et al. [22] observed an opposing trend; increasing ethanol content resulted in increased number and mass emissions, with a greater response shown by the cold engine (ECT of $20{ }^{\circ} \mathrm{C}$ ) than the warm one $\left(\mathrm{ECT}\right.$ of $\left.90{ }^{\circ} \mathrm{C}\right)$. As an example, E85 produced 15 times greater total PN concentrations and 11 times more total mass emissions than gasoline for the cold condition [22]. This is a direct contradiction to the results reported by [23]. Under cold conditions (ECT of 0-40 ${ }^{\circ} \mathrm{C}$ ), Dimou et al. [24] note that a significant increase in PM emissions below $15{ }^{\circ} \mathrm{C}$ for E0 was not seen for E10, or higher blends for that matter. The authors in this case believe that the effect of ethanol on PM formation chemistry should be the main reason for this difference, as the evaporative properties of the fuel should not have changed significantly from gasoline to E10. Chan et al. [10] found that PM emissions levels from a GDI vehicle under standard emissions drive cycles were similar for either an E0 or an E10 fuel. He et al. [29] also saw very little in terms of difference at the E0 and E10 levels, though at E20 reductions were evident.

Warey et al. [21] explored the effect of molecular structure by comparing the PM emissions from liquid fuel of two major constituents in gasoline; isooctane (a surrogate for alkanes in gasoline) and toluene (a surrogate for the aromatics in gasoline). Though they have similar boiling points, and thus similar volatility, toluene yielded greater mass and larger mean size emissions. Toluene's propensity to form soot-causing PAHs, due to its aromatic structure, was given as the reason for this finding. Two paraffin fuels were also tested, with n-pentane injection resulting in greater PM size and mass loading as compared to the emissions from the n-undecane fuel. Volatility differences were thought to be at the root of this finding; however, the phenomena of film boiling - a hindrance to evaporation - was explained to cause n-undecane (lower volatility) to evaporate more readily than n-pentane.

Gasoline fuel volatility was studied by Khalek et al. [26] by running gasoline fuel with varying grades of volatility in a GDI vehicle over standard emissions cycle tests. The fuels included certification gasoline, as well as a high volatility, cold climate gasoline and a low volatility, hot climate gasoline; the certification fuel represented the middle ground in terms of volatility. From the test results, it was found that increasing the volatility of the fuel made a marked decrease in the PM emissions. This is an expected result but still indicates some discrepancies with above mentioned results from [21].

As evidenced by this discussion, the effect of fuel on PM formation is not currently well understood. Several of the considered studies have presented conflicting results on this topic, especially at low quantities of ethanol $(<$ E10). Indeed, this is shown by the close proximity and disorder of the lines of constant ethanol in Figure 2.3. This figure also highlights that fact that work on high ethanol content blends $(\mathrm{E} 25+)$ is markedly lacking and should be pursued. This could enable a stronger argument for the use of ethanol as a PM reduction strategy in addition to its benefit at reducing greenhouse gas emissions; the initial reason behind its adoption. 


\subsection{Emissions Control Techniques}

The effect of catalytic converters on PM emissions is not well understood since modern TWCs are designed to mitigate gaseous emissions, such as $\mathrm{CO}, \mathrm{NO}_{\mathrm{x}}$, and UBHCs. In Figure 2.2, catalyzed PM emissions are typically lower in magnitude than uncatalyzed data. However, the PM reduction mechanisms in the catalyst are still largely unknown or have been speculative at best.

Whelan et al. [31] and Samuel et al. [25] studied the effect of a TWC on the emission of PM under warmed-up and cold-start idle conditions, respectively. Both studies were performed on the same production engine with a wall-guided injection system controlled by the manufacturer's originally calibrated ECU. Whelan et al. [31] saw particle size spectra shifts to larger diameters for post-catalyst emissions over pre-catalyst ones. This general observation held true for all loads examined (20-120 Nm) and shows that TWCs have profound, but different effects on the two mode of PM emissions [31]. This shift, however, was not of equal proportion, as total PN concentrations decreased in post-catalyst samples. In the cold-start study [25], total PN concentrations downstream of the catalyst were $98 \%$ less than upstream values. Furthermore, post-catalyst total particle emissions under the warm-up operation were two orders of magnitude lower than pre-catalyst values. Other studies have also looked at the effect of TWCs on PM emissions. Chen et al. [23] reported that post-catalyst PM levels (number and mass) were substantially reduced over pre-catalyst samples. He et al. [29] also reported a similar result to [23, 25, 31], where the TWC significantly reduced nucleation mode particles and slightly increased the accumulation mode at idle conditions.

Two simultaneous and conflicting processes are hypothesized to be present in the catalytic converter: (1) coagulation of particles due to small passage ways in the TWC forming larger particles, and (2) oxidation of particles leading to smaller particles [25, 31]. Consistent with these results was the observed increase in geometric mean diameter (GMD) of particles in post-catalyst samples, indicating that coagulation played an important role in the reduction of nucleation mode and increase in accumulation mode particles [31]. Engine speed was also important for this coagulation mechanism. As expected, at lower engine speeds the space velocity of the exhaust is lower than at higher engine speeds, meaning that residence time in the catalytic converter is greater at low speeds than at higher ones, thereby providing more time for the mentioned reduction methods to proceed [31]. However, both studies note that the results from this study are insufficient to provide definitive conclusions on the dominant mechanism in the catalyst $[25,31]$.

Prototype PM emissions controls (GPF) have also been considered in the literature. Chan et al. [10] tested the efficacy of a passively regenerative GPF in a production GDI vehicle over standard emissions drive cycles (FTP-75 and US06). PN concentrations were reportedly one to two orders of magnitude lower for the GPF equipped GDI vehicle than without the GPF. Moreover, adding the GPF reduced particles below $20 \mathrm{~nm}$ significantly, with $98 \%$ of $10 \mathrm{~nm}$ particles removed and $68 \%$ of $20-70 \mathrm{~nm}$ particles removed.

\subsection{Ethanol and GDI Gaseous Emissions}

Injecting the fuel directly into the cylinder also has an effect on the gaseous emissions just as it does on emitted PM. Likewise, the fuel composition also factors into the make-up of the exhaust gases. Historically, gaseous emissions work has focussed on regulated emissions ( $\mathrm{UBHC} \mathrm{NO}_{x}$, and CO); however, 
many recent studies have expanded gaseous emissions analysis to include a wider range of unregulated species. For example, harmful aldehyde emissions have been linked to ethanol combustion in several previous studies, where increasing the ethanol content of the fuel led to increases in measured aldehyde emissions [29, 33, 34, 35, 36, 37]. Further work has also been done to speciate some of the known precursors to PM formation.

Kar et al. [33] conducted an extensive investigation of organic gaseous emissions from a GDI engine under homogeneous charge operation for different ethanol blends. Total organic gas emissions (i.e UBHC emissions) in terms of $\mathrm{C}^{7}$ decreased with increasing ethanol content. Opposite to this trend, E85 emissions increased over the E50 values because evaporative cooling caused wall impinged fuel to build up in piston crevices - a known source of UBHC emissions. From an EOI timing sweep, UBHC emissions were observed to increase with either increasingly early or later EOI timings. Oh et al. [20] also observed this trend, where UBHC emissions first decreased to a minima before they increased again as the EOI timing was retarded. This UBHC emission minima was observed to increase with increasing ethanol content, due in large part to the reduction of peak combustion temperature causing a corresponding reduction in post-flame oxidation. In contrast, He et al. [29] saw no clear alcohol dependence on UBHC emissions.

Conflicting results for $\mathrm{NO}_{\mathrm{x}}$ emissions have also been seen. Some studies $[20,34,35]$ showed strong reductions in $\mathrm{NO}_{\mathrm{x}}$, while others showed no clear alcohol dependence [29]. Reductions, when found, were attributed to charge cooling effects of ethanol leading to lower peak combustion temperatures. With regards to $\mathrm{CO}$, the influence of ethanol is more clear; varying reductions with increasing ethanol have been noted in the literature $[10,29,34,38,39,35]$. Wallner and Frazee [34] argue that the presence of oxygen in the ethanol molecule promotes the oxidation of $\mathrm{CO}$ to $\mathrm{CO}_{2}$ thereby mitigating its presence in the exhaust.

Comparing GDI to PFI, Chan et al. [10] saw 4-6\% less carbon dioxide $\left(\mathrm{CO}_{2}\right)$ emissions with E0 than a similarly fuelled PFI vehicle; slightly higher reductions were noted for the E10 blend. However, CO emissions were noted to be 3-7 times higher for the GDI vehicle than on the PFI one. Both $\mathrm{CO}_{2}$ and $\mathrm{CO}$ emissions were noted to be lower when the GDI vehicle was equipped with the aforementioned GPF; the authors speculate this was related to an increase in exhaust back pressure impacting the EGR system [10].

\subsection{Sampling Techniques and Considerations}

The act of PM sampling from engine exhaust is not a trivial exercise and the manner in which the PM is extracted from the exhaust stream is very influential on the measured PM emissions. Therefore, much care is required to properly design and operate a sampling system in order to mitigate particulate losses and other erroneous results. Crucial to a PM sampling system is the process of dilution; many instruments cannot sample raw exhaust gas, as it may exceed the upper bounds of detection limits or physically overload them. Further, as previously explained in the section on PM formation and morphology, both temperature and temporal effects are important for PM formation mechanisms. The US EPA has outlined good practice and requirements for PM sampling system design in [40] and [41]. However, parameters like dilution ratio (DR), dilution temperature (DT), and dilution gas composition are left to the researcher's discretion and selected based on their needs. Many of the already considered

${ }^{7} \mathrm{C} 1$ represents a concentration made up of single carbon molecule compounds. 
studies have devoted significant amounts of work to develop and optimize a sampling technique that is partial to their needs. In particular, works by $[10,16,17,24,26]$ discuss in detail their sampling system development. The following will describe their sampling characteristics and PM measuring techniques used.

The sampling system developed by Dimou et al. [24] included a short, $85 \mathrm{~cm}$ dilution tunnel, where the raw exhaust was mixed with heated nitrogen $\left(\mathrm{N}_{2}\right)$ to achieve the desired dilution characteristics while mitigating thermophoretic losses. Both DT and DR were selected by the authors from the results of preliminary tests. They explain that a DR greater than 15 had minimal effect on the PM measured and moreover, varying the temperature between 30 to $300{ }^{\circ} \mathrm{C}$ yielded no effect on the PN size distributions. In this case, PM emissions were measured using a TSI Scanning Mobility Particle Sizer (SMPS) fed with diluted engine-out emissions sampled $15 \mathrm{~cm}$ downstream of the exhaust valve.

The exhaust in Sgro et al.'s [17] study was diluted using a one stage suction dilution system, where the engine exhaust was turbulently mixed with particle-free air at $300 \mathrm{~K}$. Sgro et al. note that above a DR of 60, particle size distributions were primarily constant in shape and magnitude; however below this point, particle distributions were shown to widen and shift to larger sizes. Furthermore, total PN and mass concentrations were shown to remain constant above a DR of 60 , which according to the authors suggests that homogeneous condensation and particle coagulation effects were suppressed [17]. Particle characterizations were made using a differential mobility analyzer (TapCon (3/150) DMA); the size distributions of charged particles were measured by replacing the normally present charge in the DMA with a "dummy volume" lacking an ion source.

Chan et al. [10] utilized a constant volume sampling (CVS) dilution tunnel in their study [10]. Two particle measuring systems were used in this investigation. The first was a European Union Particle Measurement Programme (PMP) solid particle system, where coarse particles and volatile particles were removed from the sample stream before being fed to a TSI Engine Exhaust Condensation Particle Counter (EECPC) and a TSI ultrafine CPC (UCPC). The second comprised of a TSI Engine Exhaust Particle Sizer (EEPS) fed diluted exhaust from a Dekati Thermodeneuder at $300{ }^{\circ} \mathrm{C}$ to provide PN size distributions. By decoupling the PMP and EEPS systems, Chan et al. were able to provide a higher dilution ratio to the PMP system and a lower dilution ratio to the EEPS to reduce particle losses and signal-noise issues in either system. These two particle measurement systems were found to be highly consistent with each other's results over the drive cycles and fuel combinations tested for each vehicle. Particle collection on Teflon and quartz fired filters was also performed for gravimetric and elemental-to-organic carbon ratio (EC/OC) measurements.

Another CVS system was employed by Khalek et al. [26]. The exhaust was diluted in the CVS to a variable DR in the range of 6-22; the DR was then set between the CVS and the respective instrument. A TSI EEPS and an AVL Aerosol Particle Counter (APC) were used to measure PN concentrations and size distributions, at a dilution ratio of 6 and 1000, respectively. An AVL Micro-Soot Sensor (MSS) was used to measure soot mass concentration at a dilution ratio of 2. Particle mass measurements were also made using gravimetric analysis on Teflon filters and elemental-to-organic carbon ratio analysis on quartz filters at a dilution ratio of 1.5 and a temperature of $47^{\circ} \mathrm{C}$.

For Barone et al.'s study [16] the dilution system used was similar to the European PMP, but with a lower total DR. The system consisted of two dilution tunnel stages fed by ejector pumps, with an intermediate evaporation tube stage at $350^{\circ} \mathrm{C}$. Dilution in the first stage was performed at $150{ }^{\circ} \mathrm{C}$ and at a DR of 5 , while the second stage of dilution had a lower dilution temperature of $40^{\circ} \mathrm{Cand} \mathrm{DR}$ of 6 - the 
overall dilution ratio was 30. Particle size distributions were measured using a TSI Scanning Mobility Particle Sizer (SMPS), while particle morphological analysis was carried out using transmission electron microscopy (TEM) on collected grid samples.

\section{Sampling at Low Emissions Levels}

Consideration will soon need to be given to PM sampling at decreased emissions levels that will result from the adoption of increasingly stringent emissions standards. At these emissions levels there are considerable issues with obtaining statistically relevant PM emissions measurements. Maricq et al. [42] note that filters placed in a dilution tunnel in the absence of engine exhaust still collected PM at a rate of $0.5-1.5 \mathrm{mg} / \mathrm{mi}$. The authors explain that a storage and release mechanism of particles in the exhaust lines is likely behind this observation. Bushkuhl et al. [43] also saw a similar result, where filters adsorbed on the order of $5 \mu \mathrm{g}$ of volatile material from ambient air. This is particularly troublesome when considering $20 \mu \mathrm{g}$ of PM are collected on a filter at an emission rate of $1 \mathrm{mg} / \mathrm{mi}$ during standardized testing. With this in mind, a new method of PM collection was proposed by the authors that limits this error by using a single filter to collect PM over the entire drive cycle, rather than one filter for each phase as is normally done, and uses a photoacoustic soot sensor (PASS) to apportion the collected PM to the different phases. Under this method a factor of 3-4 improvement in the level of detection over the standard method was seen $[42,43]$. 


\section{Chapter 3}

\section{Motivation and Experimental Goals}

The Engine Research and Development Lab (ERDL) at the University of Toronto has been conducting research on PM formation from GDI engines, with a particular focus on the influence of ethanol-gasoline fuel blends. Though still in relative infancy, this project has elucidated some important factors to PM formation; however, it has raised many more questions about the complex mechanisms at play.

\subsection{Previous Work}

Preliminary data gathered from a production GDI engine has shown substantial quantities of engine out PM at several operating conditions [44]. Peculiarly though, PN concentration emissions at steadystate engine (constant engine speed and load, fully warmed and steady temperatures) and instrument operation were shown to increase with run time over the course of single and multiple runs [44]. Figure 3.1 shows the results of steady-state engine testing over a 17 -day period ${ }^{1}$. Two observations are noted here: the first is the trend of increasing PM emission over single runs, and second, the general variation in PM emissions over multiple runs. The run-time based variation showed increases as high as $32 \%$ from the start to the end of the run, while run-to-run variability was as high as $37 \%$.

The observed variability was seen using different particle sizing instruments and diluters, so the observed trends were deemed to be neither a sampling nor a measurement artifact [44]. From his work, Mireault identified two potential sources of variability. Firstly, large swings in test cell humidity were hypothesized to have an effect on the stability of the dilution air from the primary diluter for the particle sizer [44]. This was thought to manifest the run-to-run variability. Secondly, injector coking was suggested as an explanation for the general increase in PM emissions with run time [44].

\subsection{Literature Revisited}

Returning to the literature and refining the scope of the present work yielded many possible explanations for the observed variabilities. Very few studies have directly identified emission variability as a problem, and even fewer have directly addressed potential sources. Inspection of data presented by Zhang and McMahon [7] show increases in PM emissions during FTP-75 standardized testing for wall guided GDI vehicles, indicating that perhaps this phenomena is hardware based and intrinsic to this apparatus.

${ }^{1}$ Data has been reported here with his permission. 


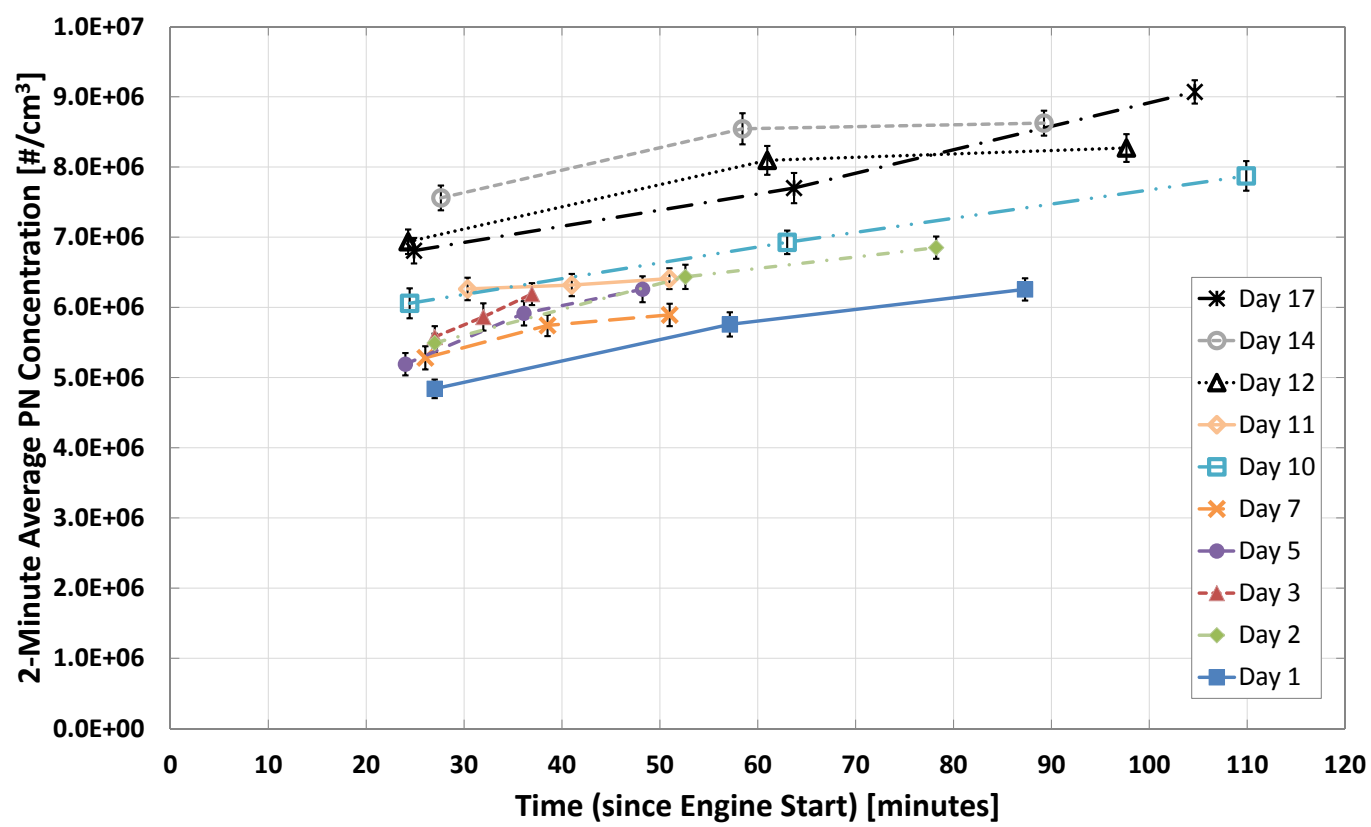

Figure 3.1: Two-minute average PN concentrations measured at the start, middle, and end of each run over 17 days [44]. Fuel was a commercially available gasoline. Error bars indicate one standard deviation.

However, the highly transient nature of this drive-cycle makes it rather difficult to say this with any degree of certainty.

The following subsections summarize key findings from the literature and previous work that may address the issues faced by Mireault [44]. These sections, in addition to the discussion presented in the previous chapter, serve as the focal point of the work performed in this investigation.

\subsubsection{Dilution Air Humidity}

As previously mentioned, Mireault identified variances in test cell humidity as a potential source of PM measurement variability. Previous studies [45, 46, 47] have shown that dilution air humidity can influence measured particle concentrations and size distributions. Rönkkö et al. [45] showed that increasing relative humidity caused increases in PN concentrations and the GMD of nucleation mode particles; accumulation mode particles remained unperturbed. Shi and Harrison [46] found a similar trend with increasing dilution air relative humidity, though an increase in larger particles was also seen. Another study also found that humid dilution air influenced measured particle concentrations, though there was no comment on particle size [47].

Test cell humidity at the time of Mireault's work was uncontrolled from the daily weather variation. This was further exacerbated by the rejection of heated cooling and dynamometer water at $40-80{ }^{\circ} \mathrm{C}$ in an open trough in the test cell during engine operation. Inspection of the diluter used for the particle sizer showed that un-dried ambient air is used to dilute the raw exhaust. Therefore, any changes in ambient humidity would also lead to changes in dilution humidity. 


\subsubsection{Engine Deposit Formation}

The secondary hypothesis presented by Mireault [44] was that injector coking (deposit formation) was influencing PM emission behaviour. Studies by Berndorfer et al. [48] and by Berkemeier et al. [49] identified injector coking (build up of a solid carbonaceous coating) as a potential source for drifting PM emissions. Berndorfer et al. specifically identified an increase in both PN concentrations and mass emissions with an increase in injector fouling. Using optical access, they were able to correlate this increase with an increase in diffusion flame counts located near the injector tip [48]. A mechanism of fuel storage in the carbonaceous material and subsequent release after the main combustion event, resulting in an observable diffusion flame, was proposed as an explanation for this observation [48, 49].

Other studies $[50,51,52,53]$ have also looked at deposit formation on GDI injectors. In a literature survey of GDI injector performance, Guthrie [50] notes that there is an injector temperature dependence on deposit formation, for both operating and soak conditions. Essentially, to mitigate formation the injector temperature needs to be either hot enough to burn off any deposit precursors or low enough to prevent fuel decomposition [50]. Additionally, the material of the injector is important to prevent fuel oxidation, especially during soak periods where liquid fuel remains in the injectors at elevated temperatures [50]. In another study, Von Bacho et al. [52] also emphasizes the importance of injector tip temperature to deposit formation; however, the properties of the fuel, specifically the $\mathrm{T} 90^{2}$ temperature, also play a critical role. In this same study, lean flow shifts ${ }^{3}$ were observed from injectors from a GDI engine that was run under a high deposit forming test regiment (run + soak) for 200 cycles. Interestingly though, lean flow shifts were reportedly not seen under continuous homogeneous operation of the engine in [52], which is analogous to the operation of this investigation's research engine.

A known issue with GDI engines is the formation of intake system deposits, primarily in the intake runners and on the intake valves. PFI engines have the benefit of washing injected fuel on the backs of these intake valves, which help cool intake valves and wash off any sources of intake valve deposits. Indeed, Parsinejad and Biggs [54] showed in recent work that GDI intake valve deposit amounts are at least one order of magnitude higher than deposits in a PFI engine. Deposits are thought to be formed from four main mechanisms [51]: (1) condensation of volatiles and deposition of PM from the EGR system; (2) condensation of volatiles from the crank case ventilation system; (3) oil from valve stem leaks; and (4) internal EGR from combustion gas back-flow. In the case of this research engine, the latter three conditions are highly applicable and could account for the production of intake valve deposits. However, very little work has been done to directly correlate intake deposits with PM production. In this light, Guthrie remarks that deposits will typically build-up to an equilibrium thickness before periodically flaking the outermost surface [50]. In any case, the substantial build-up of intake deposits is altogether detrimental to engine longevity and operation, and likely has emissions implications. Arnault and Bonne [55] propose that pleated, coalescing filters can be effective in mitigating intake deposit formation by filtering fuel and oil vapours from engine blow-by gases.

\subsubsection{Fuel and Engine Oil Temperature}

The fuelling system on the research engine was also considered as a potential source of variation in this work. Specifically, fuel temperature was found to increase over the course of a run by $10-15{ }^{\circ} \mathrm{C}$ (see Section 6.1.2) in a manner very similar to that of the PM emissions. A study by Whelan et al. [56]

${ }^{2}$ T90 - Temperature at which $90 \%$ of the fuel evaporates

${ }^{3}$ Lean Flow Shift - a reduction in fuel delivery from the required stoichiometric amount, leading to a lean condition. 
specifically looked at the PM emission impact caused by cooling the fuel to $12-23{ }^{\circ} \mathrm{C}$ below ambient temperature. Their findings showed that the chilled fuel emitted 3-13\% (depending on engine load) less total PM and shifted the distributions towards smaller sizes. Chan et al. [4] considered the influence of ambient temperature on PM emissions more generally by testing GDI vehicles at $22{ }^{\circ} \mathrm{C},-7{ }^{\circ} \mathrm{C}$, and $-18{ }^{\circ} \mathrm{C}$. Of particular interest was their finding that once fully warmed, ambient temperatures seemed to have little effect on the PM emissions from their test vehicles. This presents a contradictory finding to the one presented by Whelan et al. [56]. However a direct comparison may not be warranted as other temperature effects, such as the exhaust system, which were not independently controlled, could have influenced this finding.

\subsubsection{Engine Oil Age and Fuel Dilution}

Another potential source of variability lies with the engine lubrication oil age. A study by Christianson et al. [57] looked at the PM emissions from two PFI vehicles as their engine oil aged through drive cycle tests. They note that statistically significant changes in PM were observed as the engine oil ages. These changes were characterized first by a decrease in PM emission levels - explained as a "break-in" period - to a global minimum, followed by a subsequent rise and stabilization [57]. Retroactive analysis of Mireault's data in Figure 3.1 showed that an oil change was performed during those 17 days of testing, so this hypothesis has some foundation here. Additionally, the amount of fuel dilution (raw fuel that ends up in the bulk oil) was empirically measured in Christianson et al.'s work. Using ethanol blended fuels (E10 and E20) yielded double the amount of fuel dilution than with neat gasoline, with the former ranging in the $0.2-0.4 \%$ level [57]. Kapus et al. [58] also observed the effect of fuel dilution in an gasolineethanol fuelled GDI engine. They found up to $5 \%$ fuel dilution with gasoline fuel and interestingly, a lower 1\% fuel dilution level with an E85 fuel [58]. One thing is readily understood from the comparison of these two studies; a GDI engine should experience higher dilution levels than a PFI engine. This is easily explained by the fact that fuel directly injected into the cylinder will have greater interactions with

the oil layer on the opposing cylinder wall. Indeed, Sagawa et al. [59] found that fuel dilution tended to increase with SOI retard, which they believed to be a result of more cylinder wall exposure to the fuel spray. They also observed a fuel dilution increase with a decrease in oil and coolant temperatures. This temperature dependence could explain the conflicting findings of Kapus et al. and Christianson et al. noted above; depending on the operating temperature and run-time, more or less ethanol may be boiled out of the oil and therefore change the measured dilution level. Finally, while the correlation of fuel dilution to PM emissions is not readily shown in the literature, it could serve as a marker for cylinder wall impingement, which in itself is a leading source of PM emissions in GDI vehicles. This has the added implication of oil ingress into the combustion process by spray interactions with cylinder oil layer, which was shown by Berkemeier et al. [49] to be a contributing factor to the amount of PM emission observed.

\subsubsection{Fuel Effects}

The previous chapter offers a detailed look at the influence of ethanol on PM emissions from GDI engines. As noted, while some investigations note decreases in PM emissions with increased ethanol content $[24,60,61]$, others have shown the opposite trend $[4,22]$, and others still are indecisive on the matter $[10,23,28,29,57]$. It is clear that oxygenate fuel blends have produced conflicting results and 
as a result, other fuel properties have been identified as playing important roles also.

The previously mentioned study by Warey et al. [21] identified toluene's propensity to form PAH compounds as a key contributor to soot production in GDI engines. In an investigation on the influence of ethanol, Vuk and Vander Griend [60] found high boiling point aromatics to be the primary factor responsible for PM formation in GDI engines. In their analysis, they provide that using ethanol promotes evaporation and significantly reduces PM produced by fuels with high boiling point aromatics [60]. A similar finding was given in the work of Khalek et al. [26], where commercial fuels with the greatest amount of high boiling point compounds (such as double-bonded alkenes and aromatic compounds) emitted the most PM. Beyond the molecular structure of the fuels, these studies have also identified another influential fuel parameter to PM production; fuel volatility. Khalek et al. state that of the three commercial fuels they tested, the fuel with the highest volatility emitted the lowest PN concentrations, and vice-versa. Kim et al. [62] compared three fuels with varying aromatic content and vapour pressure in their investigation. They also found that fuels with higher aromatic fractions and lower volatility resulted in the highest PM emissions. Additionally, a couple of studies [60,62] have identified that certification fuels, such as Indolene, are often much cleaner than commercially available fuel in terms of PM production. This has implications for emissions certification in that production vehicles in the field may exceed emissions limits even if they meet them in certification conditions.

With this in mind, two studies have attempted to develop predictive models and PM indices with the hopes of better predicting the emissions of commercially available fuels. Aikawa et al. [63] first developed this PM index, which was subsequently explored in a follow-up study by Leach et al. [64]. In both of these works, the dependence on the amount of double-bond (or higher) constituents and the fuel's overall vapour pressure were identified as the most influential parameters, which mirrors the above discussion. In both cases a fuel based PM index was developed, taking the double-bond equivalent (DBE) value of each constituent in the fuel and their corresponding vapour pressures into account $[63,64]$. Note that small differences in the two equations are present, namely the derivation of the vapour pressure and fractional weighting (mass vs. volume); however, the overall behaviour is the same - see Equations 3.1 and 3.2 .

Aikawa et al. [63]:

$$
\text { PMIndex }=\sum_{i=1}^{n}\left(\frac{D B E_{i}+1}{V P(443 K)_{i}} \times W t_{i}\right)
$$

Leach et al. [64]:

$$
\text { PNIndex }=\frac{\sum_{i=1}^{n}\left(D B E_{i}+1\right) V_{i}}{D V P E} \times 100 k P a
$$

where:

$i$ - fuel constituent

$W t_{i}$ - mass fraction of fuel constituent $i$

$V_{i}$ - volume fraction of fuel constituent $i$

$V P(443 K)_{i}$ - absolute vapour pressure of fuel constituent $i$ evaluated at $443 \mathrm{~K}$

$D V P E$ - Reid Vapour Pressure $(\mathrm{kPa})$ at $311 \mathrm{~K}$ for the fuel mixture

$D B E_{i}=\frac{2 C-H-N+2}{2}-$ double-bonded equivalent is a measure of how unsaturated the fuel constituent is $(\mathrm{C}, \mathrm{H}$, and $\mathrm{N}$ are the number of atoms present in the compound) 
Aikawa et al. [63] found excellent agreement between predicted indices and actual PM mass and number emissions from a PFI vehicle. An analysis of commercially available fuels world-wide indicated that PM indices can vary by as much as a factor of 10 [63]. The work of Leach et al. [64] likewise found agreement between predicted indices to custom blended fuels with varying aromatic fractions and vapour pressures. Therefore, given that Mireault's work - as well as the work in this thesis - was performed on commercially available gasoline it is possible that variances in fuel composition caused variances in engine out PM emissions.

\subsection{Experimental Goals}

The overarching goal of this thesis is to identify, explain, and if possible control the sources of variability in PM emission insofar as to improve the repeatability of results. This will allow future work on this apparatus to better analyze the true influence that ethanol in gasoline has on PM emissions, which of course forms the crux of this project. Based on the above discussion of literature results, and from previous work on this engine, the following areas of interest were selected for investigation.

- Control of dilution air humidity on PM emissions.

- Control of engine lubrication oil and fuel temperatures on PM emissions.

- Influence of induction system (intake manifold, intake ports, and intake valves) and engine deposits on PM emissions.

- Influence of engine lubrication oil age and fuel dilution effects on PM emissions.

- Influence of fuel properties from fuel blends with varying amounts of ethanol and toluene, including different suppliers of commercially available gasoline on PM emissions. 


\section{Chapter 4}

\section{Experimental Set-up}

The following chapter elaborates on the physical apparatus used for the research conducted. Figure 4.1 shows a schematic diagram of the equipment set-up and should provide the reader with an overview. The set-up is largely similar to the one first presented by Mireault in his thesis [44]; however, several modifications have been made and are duly noted in the proceeding sections. Appendix A contains supplementary information relevant to this chapter.

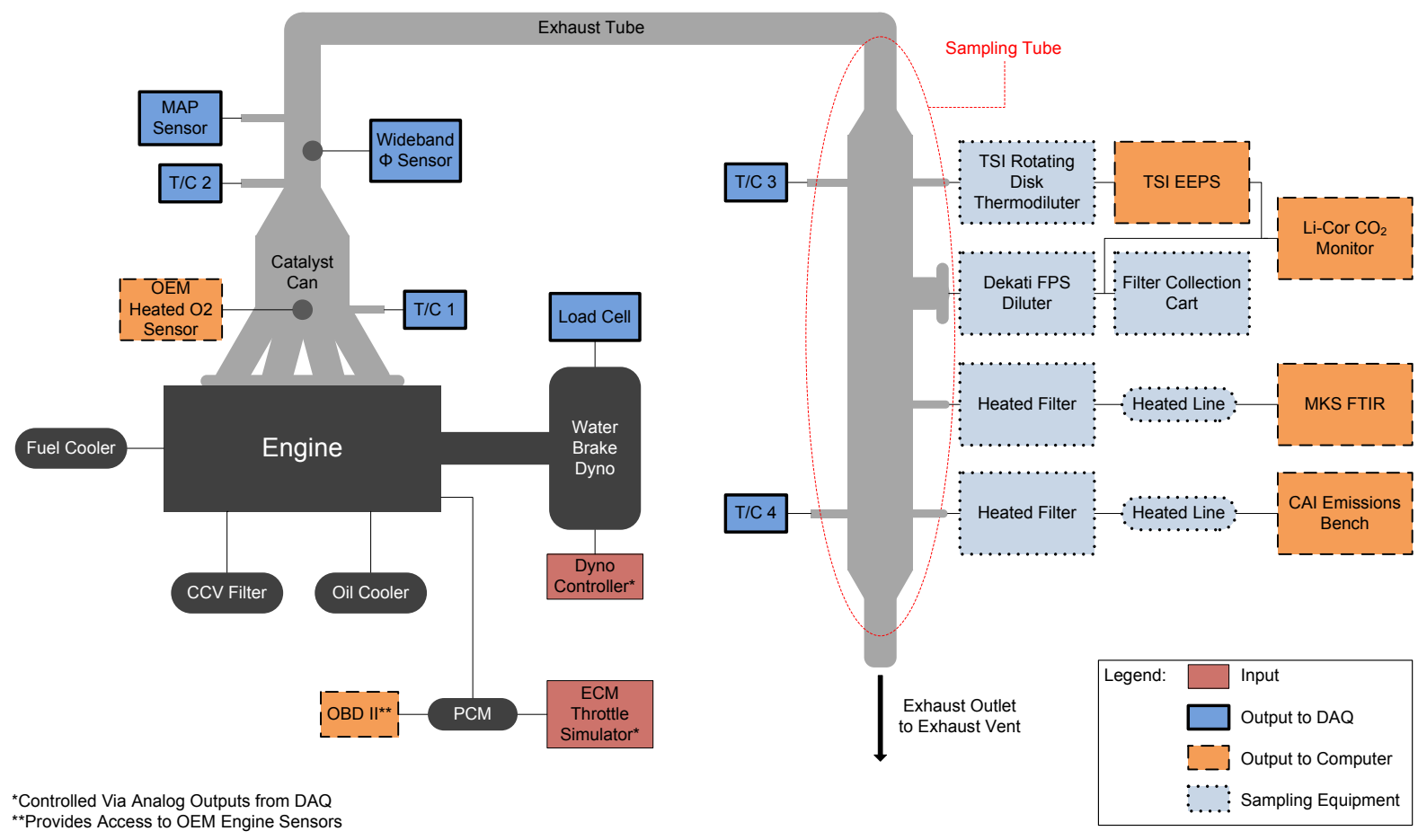

Figure 4.1: Research engine and emissions sampling arrangement.

\subsection{Research Engine}

The engine used in this research is a pre-production four-cylinder GDI engine used in the 2012 and newer Ford Focus. It employs side mounted, wall-guided direct injectors and is naturally aspirated. Table 
4.1 lists some of the major specifications of the engine. A stock ECU-known as a Powertrain Control Module (PCM) with Ford - electronically controls the operation of the engine, and in production vehicles the transmission and other drivetrain components. It has the ability to read on-board sensor data, look up predetermined engine maps, and adjust them according to the current conditions. Parameters such as spark timing, injection timing, valve timing, and many more, are directly controlled by this unit and can be varied in real-time. A custom dyno-wiring harness interfaces the ERDL-made engine control panel with the stock PCM and engine wiring harness.

Table 4.1: Research engine specifications [65].

\begin{tabular}{ll}
\hline Displacement & $1999 \mathrm{~cm}^{3}$ \\
Bore x Stroke & $3.44 \times 3.27 \mathrm{in}$. \\
Compression Ratio $\left(\mathrm{r}_{c}\right)$ & $12.0: 1$ \\
Horsepower & $160 @ 6500 \mathrm{rpm}$ \\
Torque & $146 \mathrm{ft}-1 \mathrm{~b} @ 4450 \mathrm{rpm}$ \\
Redline & $7000 \mathrm{rpm}$ \\
Fuel Injection & Direct Gasoline Injection \\
Valvetrain & Double overhead camshafts (DOHC) \\
& Four valves per cylinder \\
Block and Head Material & Twin independent variable camshaft timing (Ti-VCT) \\
Recommend Fuel & Aluminium \\
Emissions Control & 87 Octane \\
Emissions Standards & Three-way catalyst \\
\hline
\end{tabular}

\subsubsection{Powertrain Control Module}

The PCM was supplied with a non-production engine calibration. Discussion with Ford has indicated that this PCM was used in durability testing campaigns and has a slightly rich tune and is not configured for proper catalyst function. Indeed, wide-band oxygen sensor readings showed a fuel-air equivalence ratio of approximately 1.015 at steady conditions. However, the exact calibration of the PCM is not known and it is currently unmodifiable.

\subsubsection{Engine Exhaust System}

A standard equipment exhaust manifold was used in this set-up, which normally has an incorporated TWC - see Figure 4.1. However, given that the PCM is not configured to run with a TWC, the exhaust manifold was modified to remove the stock exhaust after-treatment. This was accomplished by cutting open the catalyst can, removing the catalytic core and re-welding the can back together. Keeping an otherwise stock exhaust manifold will permit future work on this engine to look at catalyzed vs. uncatalyzed emissions under an identical set-up. The exhaust manifold was joined to the exhaust tube, which in turn feeds the raw engine exhaust to the sampling tube at a typical tail-pipe distance. The exhaust system and sampling tube were designed in accordance to US EPA guidelines [40]. 


\subsubsection{Fuelling System}

A custom fuel system was implemented here, as the use of a stock fuel system would not be appropriate. Figure 4.2 shows a schematic diagram of the fuelling system. Two fuel tanks hold the test fuel and are selected via two three-way solenoid valves, which control the flow path of the outgoing and return fuel. A coarse filter removes large debris from the flow to protect the low pressure fuel pump, while a fine filter is employed to prevent any smaller debris from entering the high pressure loop on the engine side. A pressure regulator ensures that fuel at $55 \mathrm{psi}$ is continuously supplied to the high pressure fuel pump, with unused fuel being returned to the appropriate tank. A fuel cooler was added in this investigation to control the fuel temperature being fed to the high pressure loop. The fuel temperature is measured at the exit of the pressure regulator before the fine filter. The fuel cooler is described in greater detail in Section 4.2.1.

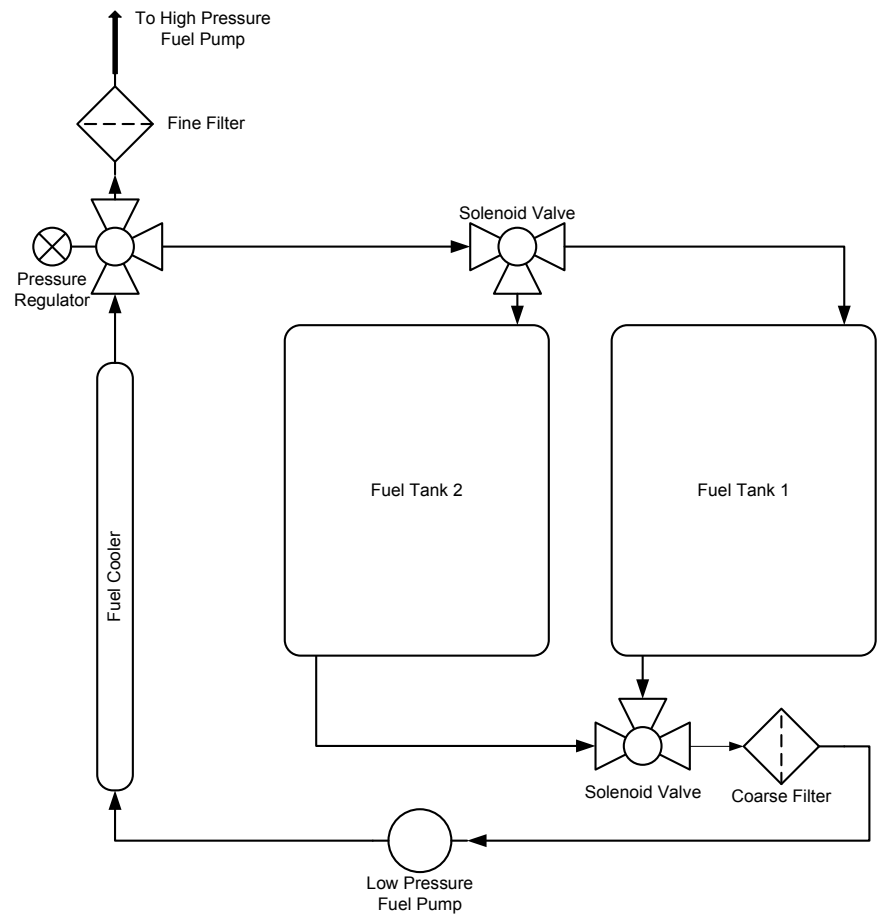

Figure 4.2: Fuelling system flow diagram.

\subsubsection{Dynamometer}

A GO-Power D-557 water brake dynamometer was used to apply a load on the engine. The water brake dynamometer works by transferring the mechanical energy of the engine to the water due to friction and turbulence generated between the rotor and stator. The rotor is coupled to the engine, while the stator is coupled to the dynamometer stand. A strain gauge measures the force applied to the stator by the rotating fluid in the internal cavity. Water is supplied at a pressure that is regulated down from building pressure; the amount of water flowing to the dynamometer is controlled via the dynamometer controller (see Section 4.3.2). 


\subsection{Recent Engine Modifications}

Three major modifications were made to the research engine for this particular work: (1) a fuel cooler was added to maintain a steady fuel temperature in the low-pressure loop; (2) an oil cooler was added to observe the effect of lowering the steady-state temperature of the oil and reduce the engine oil warm-up time; (3) and a crankcase ventilation filter was added to reduce the ingestion of oil into the induction system, thereby mitigating the formation of intake deposits. These modifications are elaborated upon in the following subsections.

\subsubsection{Fuel Cooler}

A custom fuel-to-water cooler was made to provide control over the fuel temperature. Figure 4.3 shows a 3-dimensional render of the fuel cooler, which is composed of standard pipe and compression fittings. Measured in-tank fuel temperature was found to increase steadily with run time (see Section 6.1.2), due to the fact that the low pressure fuel pump is cooled by the fuel that is continuously pumped in the low pressure loop. Figure 4.4 shows a cut-away view of the fuel cooler. The custom fuel cooler amounts to a single shell, single tube heat exchanger. With an annular cross-section, fuel is pumped through the centrally mounted tube, while city water is counter-flowed through the shell side. A ball valve controls the water flow upstream of the cooler, while a precision needle valve regulates the outflow of water from the cooler. The ball valve allows the user to disable the cooler and the precision needle valve controls the fuel temperature.

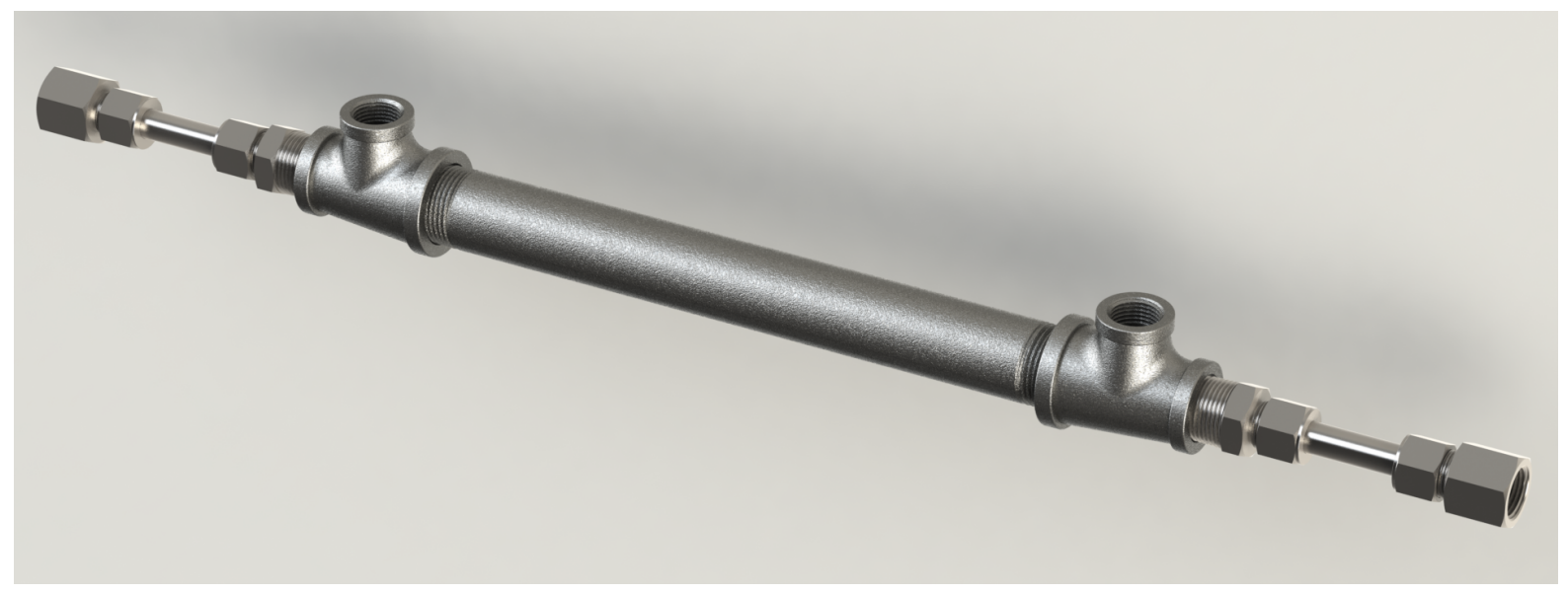

Figure 4.3: 3D render of fuel cooler.

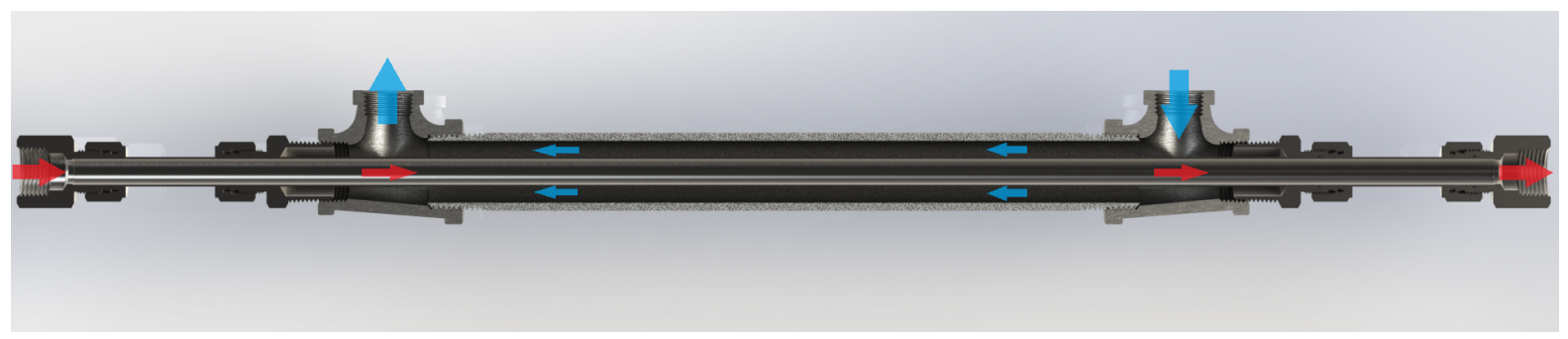

Figure 4.4: Cross-sectional view of fuel cooler. Red arrows indicate fuel flow path and blue arrows indicate water flow path. 


\subsubsection{Oil Cooler}

A sandwich type oil cooler from a 2012 Ford Focus ST (turbocharged, high performance variant of this research engine) was installed for this work. Figure 4.5 shows the installation location and the relevant flow paths. The oil cooler works by flowing engine coolant through a heat exchanger that is seated above the oil filter. Pressurized oil is forced through this cooler before continuing through the filter and back to the engine block. The oil cooler was installed in the typical coolant loop location for the heater core ${ }^{1}$ on a production vehicle. This location ensures that coolant flows through the oil cooler at all times while the engine is turning over because the coolant path for the heater core is unobstructed by the thermostat. By having coolant flowing at all times, the cooler works to both heat the oil more quickly in cold conditions and cool the oil when the engine is fully warmed. A ball valve downstream of the oil cooler allows disabling of the unit by preventing coolant flow to it.

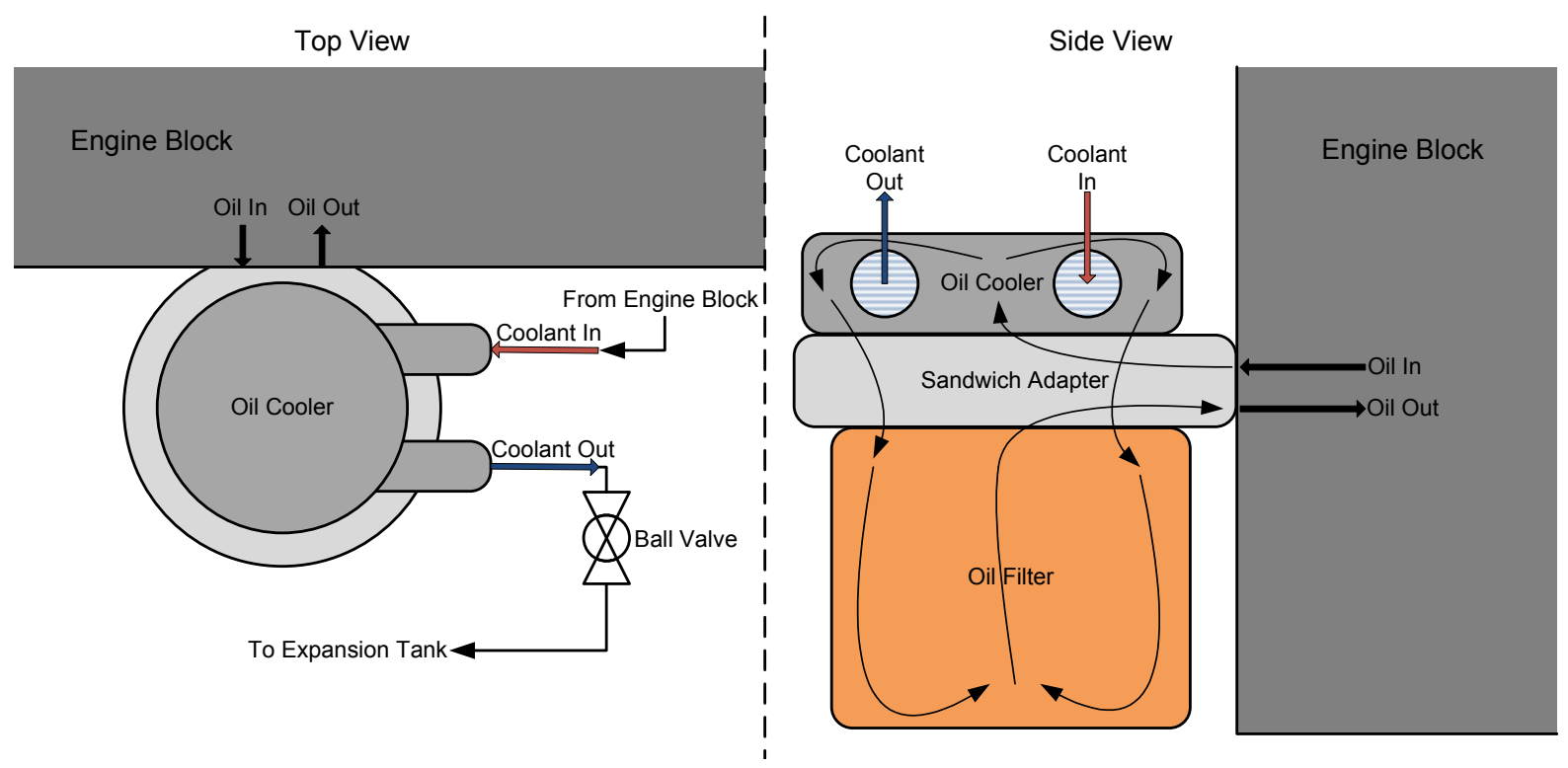

Figure 4.5: Schematic of oil cooler. Note: oil flow paths do not intersect.

\subsubsection{Crankcase Ventilation Filter}

A crankcase ventilation system is designed to recycle engine blow-by gases ${ }^{2}$ back into the induction system of the engine so that it may be combusted with incoming fuel-air charge. This prevents the uncontrolled emission of these gases to the atmosphere, and is part of emissions control packages on vehicles. In the case of this engine, a positive crankcase ventilation (PCV) system is utilized; vacuum generated by the engine in the intake manifold draws in the crankcase air and blow-by gases. This crankcase ventilation is regulated by a PCV valve that also reduces the vacuum from inHg in the manifold to $\mathrm{inH}_{2} \mathrm{O}$ in the crankcase. The stock engine design incorporates an oil separator onto the engine block that functionally prohibits the ingestion of crankcase oil into the induction system via this ventilation system. However, inspection of the intake manifold and runners showed that some diluted

\footnotetext{
${ }^{1} \mathrm{~A}$ heater core is a heat exchanger that heats air for the passenger compartment.

${ }^{2}$ Blow-by gases are products of combustion and unburnt fuel vapours that escape the combustion chamber through the piston rings.
} 
engine oil had escaped this separator-Figure 4.6 shows the oil collected in the intake manifold. As a result, a MANN+HUMMEL ProVent200 oil separator filter for closed crankcase ventilation was installed. An exploded diagram of the filter is provided in Figure 4.7.

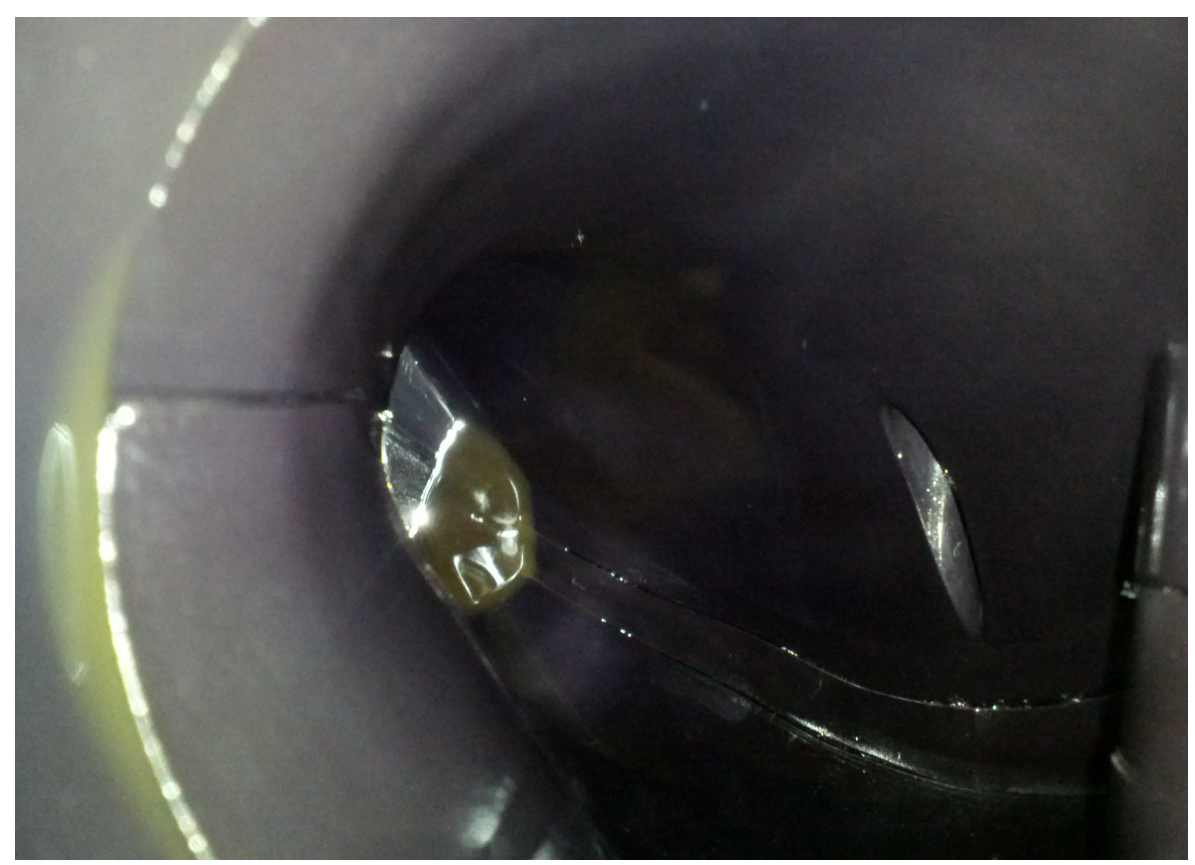

Figure 4.6: Collected oil in the intake manifold - image taken from the perspective of the throttle body. Note that the manifold is shown rotated to purposely collect oil at far side of plenum to indicate relative quantity of oil.

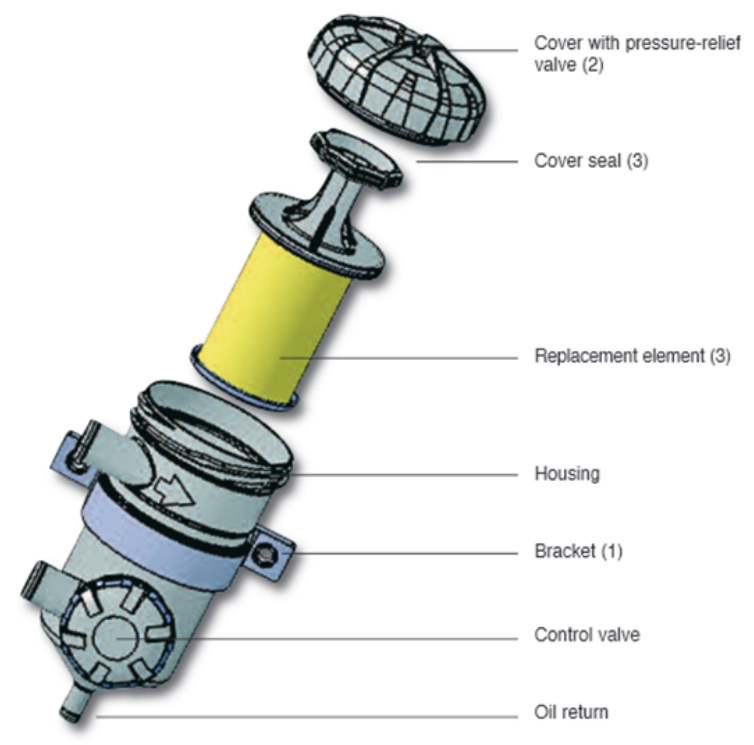

Figure 4.7: Exploded view of the MANN+HUMMEL ProVent200 oil separator filter [66].

The filter was fitted downstream of the stock PCV valve and upstream of the intake manifold. A coalescing filter element absorbs oil vapours and liquid oil being pulled by the vacuum of the intake manifold. Once the filter begins to saturate, the oil collects at the bottom of the filter housing where 
it normally drains back to the oil sump. As this was not required for this situation, a simple pet-cock valve was installed to allow collected oil to drain out between tests.

The filter housing contains two non-adjustable valves to protect the system from excessive positive pressure and to regulate the flow of blow-by gases. Since the typical application for this filter is in diesel engines that are often turbocharged and may not incorporate PCV valves, the unit is not designed to handle the typical vacuum generated by a naturally aspirated spark-ignited engine. Preliminary runs with the filter installed produced a series of vacuum leaks through the unit and caused the PCM to trim the commanded fuel to very rich levels. The control valve membrane was found to be failing under the large vacuum so a solid aluminium plug was machined and installed in its place - see Appendix A.1 for drawing. The stock PCV valve incorporated into the oil separator on the engine block sufficiently regulates the flow of blow-by gases in this case.

\subsection{Engine Controls}

A custom made engine control panel operates the major electrical control functions for the engine. These include the starting system, the fuelling system, the PCM system, and the emergency stop system. The control panel is the same one used in the first study on this engine by Mireault [44]; specific details are omitted here for the sake of brevity.

\subsubsection{Throttle Control}

An ECM appsCAN throttle pedal simulator was used to simulate the throttle pedal signals to the PCM. Modern vehicles make use of throttle-by-wire systems, where an electrical sensor measures the throttle position at the pedal and relays that to the PCM. The PCM then performs the necessary calculations to select the appropriate throttle plate angle given the commanded throttle position and other critical parameters; the PCM physically controls the throttle plate angle with a servo motor. As owner safety is of utmost importance to auto manufacturers, the throttle pedal actually has two sensors that must mathematically correspond to each other; the PCM reads both values and should one not correspond to the other a fault is triggered and the vehicle enters what is known as "limp-mode". Therefore, the coordination of these two signals is necessary for proper engine operation. The appsCAN provides a synchronous output of signals in a stand-alone fashion required to operate the throttle [67]. This throttle pedal simulator represents a modification from the original throttle control used by Mireault [44], which amounted to a servo-controlled throttle pedal. The need for this change was borne out of improving the repeatability of the throttle control process, which would be a requirement for future drive cycle work. Therefore, the appsCAN module was installed to satisfy this requirement as well as to better automate the control of the research engine.

Specific to this research engine, the throttle pedal outputs two position signals, APPS1 and APPS2, based on its current position to the PCM. Measurement of the signals showed that APPS2 is $50 \%$ of APPS1 at any given position. As such, the appsCAN module was set to run in ratiometric mode, where the two desired outputs would run at a fixed percentage of an input voltage signal. A $0-10 \mathrm{~V}$ input signal from the NI DAQ (see Section 4.4.1) was used to control the appsCAN which in turn provided two signals at $50 \%$ and $25 \%$ of the original input. Note that the signal was scaled in the Labview program to fit the required signal range of the pedal to a $0-100 \%$ scale (i.e. a $0 \%$ selection in the program would yield a $1.6 \mathrm{~V}$ output to the module, which corresponds to the $0 \%$ pedal position for sensors APPS1 and 
APPS2). Further logic was incorporated to read throttle ramps from a file, as well as provide for certain fail safes.

\subsubsection{Dynamometer Control}

A Digalog 1022A Dyno Controller was used to provide control of the water brake dynamometer mentioned in Section 4.1.4. The dyno controller reads engine speed from a magnetic pick up on the dynamometer, as well as the strain voltage (calibrated for torque loading) from the strain gauge. The dyno controller has the ability to control the load and speed set-points given these two readings by controlling the amount of water entering the dynamometer through a compressed air controlled water valve. Essentially, this works by regulating the amount of compressed air supplied to open the water control valve. This is done with an on-board closed loop PID controller, which was re-calibrated along with the stain-gauge prior to start of this work. In this investigation, the controller was set to control the engine speed, the set-point of which was controlled by analog voltage signals from the NI DAQ (see Section 4.4.1).

\subsection{Engine Data Acquisition and Control}

Critical engine parameters, temperatures, and pressures were recorded using two independent data acquisition systems. Engine specific parameters were recorded using the high-speed controller area network (CAN) bus that is standard to production vehicles and known as the on-board diagnostic version 2 (OBD-II). The other acquisition unit - a National Instruments CompactDAQ - measured temperatures, pressures, and other anolog signals, as well as outputted control signals. This data stream was recorded using a modified version of the Labview program used by Mireault [44].

\subsubsection{National Instruments Compact DAQ}

A National Instruments (NI) CompactDAQ (cDAQ-9174) populated with four modules captured analog data such as temperature, pressure, and load. Two NI-9211 thermocouple modules provided acquisition of temperature data, while an NI-9205 module collected analog sensor data. A single NI-9263 module handled analog outputs used to control the throttle simulator (Section 4.3.1) and the dyno controller (Section 4.3.2) for the load and speed set-points, respectively. Table 4.2 lists all the sensor data collected and the analog outputs controlled by the DAQ. All thermocouples used were Omega K-Type grounded thermocouples (HGKQSS-18G-12). Pressure measurements were made with engine manifold air pressure sensors (MSD Ignition 2312).

Two independent Labview programs were used with the DAQ for data recording and output control. The main program for recording purposes is a modified version of the original program used by Mireault [44]. The above inputs from the NI DAQ, as well as inputs from the standard emissions bench (Section 4.6.3) over $\mathrm{TCP} / \mathrm{IP}$, are read and written to a file at $5 \mathrm{~Hz}$ and $1 \mathrm{~Hz}$, respectively. Modifications to this program include the addition of the previously excluded emissions analyzers and the calculation of corrected emissions from the standard emissions bench. The second program controls the throttle (load) and engine speed set-points for the throttle and dyno controllers, respectively. Manual control or pre-developed load and speed ramps are available for selection by the user. 
Table 4.2: Inputs and outputs from NI DAQ. Units shown are appropriately scaled from analog voltages and currents.

\begin{tabular}{ccll}
\hline & NI Module & \multicolumn{2}{c}{ Recorded/Outputted Parameter } \\
\hline & & TC1 - Exh. Temperature $\left({ }^{\circ} \mathrm{C}\right)$ & TC2 - Exh. Temperature $\left({ }^{\circ} \mathrm{C}\right)$ \\
& $9211(2)$ & TC3 - Exh. Temperature $\left({ }^{\circ} \mathrm{C}\right)$ & TC4 - Exh. Temperature $\left({ }^{\circ} \mathrm{C}\right)$ \\
Inputs & & Ambient Air Temperature $\left({ }^{\circ} \mathrm{C}\right)$ & TSI Diluter Head Temperature $\left({ }^{\circ} \mathrm{C}\right)$ \\
& & Oil Temperature $\left({ }^{\circ} \mathrm{C}\right)$ & Fuel Temperature $\left({ }^{\circ} \mathrm{C}\right)$ \\
\cline { 2 - 4 } & \multirow{3}{*}{9205} & Abs. Ambient Pressure $(\mathrm{kPa})$ & Abs. Exh. Pressure $(\mathrm{kPa})$ \\
& & Engine Speed $(\mathrm{rpm})$ & Engine Load $(\mathrm{ft}-\mathrm{lb})$ \\
& & Fuel-Air Equivalence Ratio $(\phi)$ & Engine Speed Set-Point $(\mathrm{rpm})$ \\
\hline Outputs & 9263 & Throttle Position $(\%)$ & Engine Speed Set-point $(\mathrm{rpm})$ \\
\hline
\end{tabular}

\subsubsection{OBD-II}

Data from the OBD-II CAN bus was gathered using a generic after market OBD-II reader. The reader contains an ELM-327 chip which is widely used to decode CAN messages over the OBD-II protocolknown as parameter IDs (PIDs) - and interface with computer software via USB. As the OBD-II standard is made to provide a minimal level of access to diagnose issues with vehicles, this chip can only decode those CAN values deemed essential for troubleshooting by regulatory agencies. However, manufacturers do employ proprietary CAN messages which are only decodable with proprietary readers. A list of the available PIDs recorded during this investigation is provided in Table 4.3.

Table 4.3: PIDs recorded from OBD-II data stream.

\begin{tabular}{ll}
\hline Engine Speed $(\mathrm{rpm})$ & Engine Coolant Temperature $\left({ }^{\circ} \mathrm{C}\right)$ \\
Intake Air Temperature $\left({ }^{\circ} \mathrm{C}\right)$ & Calculated Load $(\%)$ \\
Air Flow Rate $(\mathrm{g} / \mathrm{s})$ & Ignition Timing Advance $\left({ }^{\circ}\right)$ \\
Accelerator Pedal Position $(\%)$ & Absolute Throttle Position $(\%)$ \\
Fuel Rail Pressure $(\mathrm{kPa})$ & Air-Fuel Equivalence Ratio $(\lambda)$ \\
Long Term Fuel Trim $(\%)$ & Short Term Fuel Trim $(\%)$ \\
\hline
\end{tabular}

\subsection{Particulate Matter Sampling}

The following section will detail the particulate matter sampling apparatus used in this investigation. The two dilution systems are discussed first, followed by the instruments used to analyze size, mass, and composition of the particles. All referenced instruments were serviced at acceptable intervals prior to and during data collection per manufacturer recommendations.

\subsubsection{TSI Rotating Disk Thermodiluter}

A TSI 379020A Rotating Disk Thermodiluter supplied diluted exhaust gas to the particle sizer used in this study (see Section 4.5.4). The 379020A is controlled and fed dilution air from a combination of a 
TSI 379030 Thermal Conditioner Air Supply and a Matter Engineering MD19-3E Raw Gas Diluter; this latter unit will henceforth be referred to as the 'diluter box'. This diluter is capable of achieving DRs between 15:1 to $3300: 1$ at flow rates between $0.5-20 \mathrm{lpm}$ [68]. The 10-cavity rotating disk mixes fixed volumes of exhaust gas with the primary dilution air [68]. The par-diluted sample is sent to a thermal conditioner and thermodenuder, before being cooled by the secondary dilution [68]. Through results obtained by Mireault, the diluter was found to produce stable DRs; however, true DRs were found to be consistently under the set-point desired. As a result, the true dilution ratio was measured using $\mathrm{CO}_{2}$ as a tracer gas.

\section{Diluter Box Modification}

The factory configuration of the diluter box draws ambient air through HEPA filters to condition the dilution air. Visual inspection of the unit showed no form of air drying. As dilution air humidity is thought to be a source of variability in PM measurement (refer to Section 3.2.1), modifications were made to the diluter box in order to accept a dry supply of dilution air. This consisted of plumbing flexible tygon tubing from the HEPA filter inlets to the top of the unit, where a connection was made to a line of dry pressurized air (see Section 4.5.3). The pressurized air is supplied at ambient pressure by venting any excess to ambient.

\subsubsection{Dekati FPS 4000 Diluter}

The Dekati Fine Particle Sampler (FPS) 4000 provided diluted exhaust sample for the filter collection cart (Section 4.5.5). The unit is capable of supplying 60-160 lpm of diluted sample at DRs of 20-200 [69]. The Dekati incorporates two stages of dilution to achieve the desired dilution ratio. The first stage of dilution occurs through a perforated tube, where the dilution air is drawn into the sample stream through a porous metal tube; the second stage is an ejector type diluter [69]. The DR is controlled by the amount of dilution air flowed, which the unit controls via several solenoid valves and critical orifices [69]. A primary dilution air heater and a dilution probe heater allow for adequate DT control. A calculated dilution ratio is provided by on-line measurement of temperature and pressures through the system, though its accuracy has been put into question by previous work at the ERDL [44, 70, 71]. Therefore, as with the rotating disk thermodiluter, the true dilution ratio was measured using $\mathrm{CO}_{2}$ as a tracer gas.

\subsubsection{Dry Air Supply}

Dry dilution air was supplied to both the Dekati diluter and the rotating disk thermodiluter via a system of filters and desiccant driers on the building's pressurized air line. This dry air supply meets or exceeds the specifications required by the Dekati. A full listing of specifications of the dry dilution air system is provided in Appendix A.3. This dry air supply was also used to provide the dry air needed for the emissions bench (HFID and HCLD analyzers) - see Section 4.6.3. All filters were replaced and the desiccant was re-dried prior to the start of this work. 


\subsubsection{Engine Exhaust Particle Sizer}

A TSI 3090 Engine Exhaust Particle Sizer (EEPS) provided total PN concentrations and particle size distribution characterizations of the engine exhaust. Particles are characterized by their electrical mobility in 32 discrete bin sizes from 5.6 to $560 \mathrm{~nm}$ [72]. The EEPS works by charging incoming exhaust particles to a known level with a corona charger [72]. The charged particles then flow through the column with a centrally mounted, positively charged electrode repelling the particles radially where the electrometers are located. The electrometer that any given particle impacts is related to its electrical mobility and therefore, its particle size. These electrometers measure the charge of the impacted particle allowing both size and concentration data to be extracted simultaneously [72]. Data was logged using the proprietary TSI software at a rate of $2 \mathrm{~Hz}$. The EEPS measured diluted exhaust from the aforementioned TSI Rotating Disk Diluter (Section 4.5.1).

\subsubsection{PM Filter Collection Cart}

An ERDL designed and built filter collection cart was used in this study to collect PM laden filters for gravimetric and compositional analyses. The filter cart was first used in [70, 71] and later in [44]. Figure 4.6 shows a schematic configuration of the filter collection cart. Diluted exhaust sample is drawn through the filter cart via two vacuum pumps - one for each filter cassette holder. A cyclone removes particles greater than $10 \mu \mathrm{m}$ upstream of the filter holders, which hold the filter cassettes that collect the PM on each filter. Sample collection is controlled by two, three-way solenoid valves, which when selected to sample connect the exhaust flow path through the filter cassette holders.

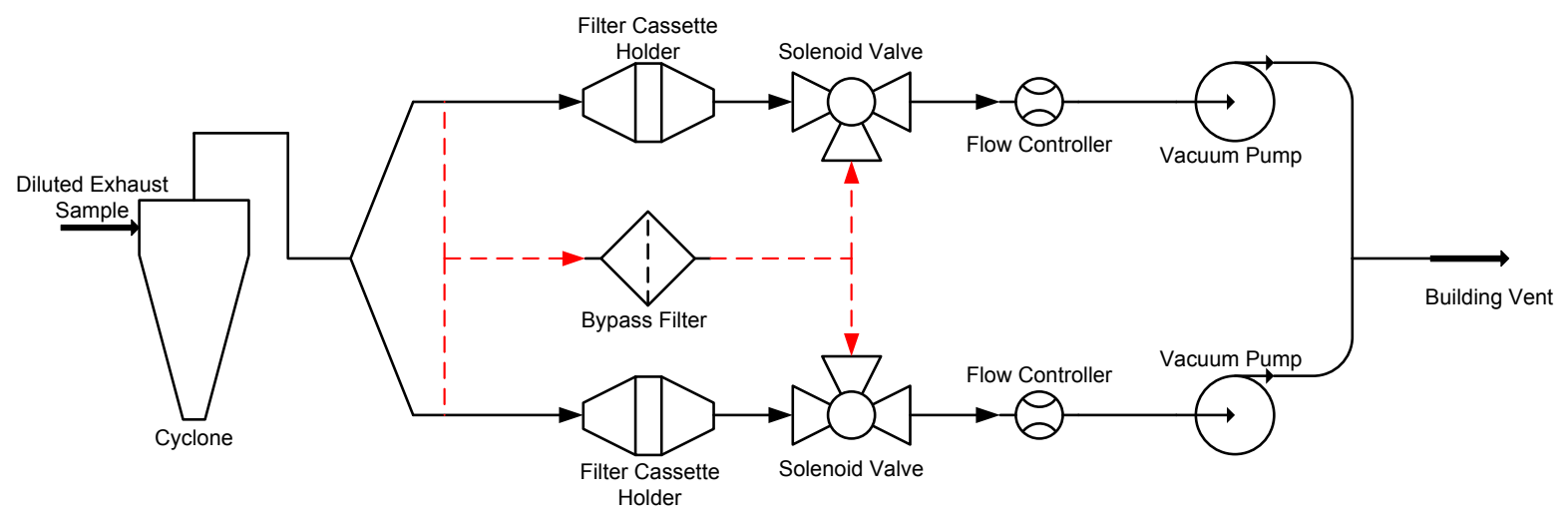

Figure 4.8: Filter cart flow diagram. Dashed lines indicate flow path in "bypass" mode.

\section{Filter Cart Modifications}

The filter cart was modified for this investigation based on the recommendations of Mireault [44]. The Dekati diluter is highly back-pressure sensitive, and the act of switching the solenoid valve to sample on the filter cart was found to cause too high a pressure fluctuation and would perturb the dilution characteristics. As a result, the flow path of the filter cart was altered to include a bypass section, whereby the sample pumps would continuously pull on the Dekati diluter irrespective of the solenoid valve position. A Parker-Balston 58N Engine Exhaust Filter (with a 100-12-404 filter element) was 
installed in this bypass flow path to remove particles and to protect the mass flow controllers when in this configuration.

A custom heater wrap was also added in order to achieve the US EPA post filter temperature requirement of $47 \pm 5{ }^{\circ} \mathrm{C}$ [73]. A Neptech Inc. Hot Pocket custom heater blanket (52C-AN-CUSTOM$\mathrm{KN}-\mathrm{V}$ ) was installed on the cyclone separator to help keep the exhaust sample in this temperature range. The Hot Pocket was custom sewn for the cyclone shape and incorporates a heater cord in an insulation wrap. An Omega CN7533 PID temperature controller controls the temperature set-point of the hot pocket with feedback from an embedded thermocouple.

\subsubsection{Gravimetric Filter Analysis}

Gravimetric filter analysis was conducted according to the procedure detailed in the US EPA's Standard Operating Procedure [74]. All gravimetric measurements were conducted in a class 100 clean room using a Satorius SE-2F Microbalance, which meets the US EPA specifications. NIST traceable calibration weights were used to verify the accuracy of the microbalance on an ongoing basis. An electrostatic neutraliser was used to neutralise filter samples prior to taking measurements. The clean room was maintained at a temperature of $22 \pm 1{ }^{\circ} \mathrm{C}$ and a relative humidity of $45 \pm 5 \%$.

\subsubsection{Compositional Filter Analysis}

Elemental-to-organic carbon ratio (EC/OC) analysis was performed using a Sunset Thermal-Optical Semi Continuous OC/EC analyzer. The analyzer operates by thermally desorbing and oxidizing organic and elemental carbon species, respectively, from a PM laden quartz filter. First, organic compounds are thermally desorbed from the filter in an inert gas environment by heating the filter incrementally. This carbon is then oxidized to form $\mathrm{CO}_{2}$ in a manganese dioxide oven, which is measured by a non-dispersive infrared detector (see Section 4.6.3 for a description of this type of analyzer) [75]. The second stage of the analysis is performed with an oxidizing gas so that the elemental carbon forms $\mathrm{CO}_{2}$ [75]. Note that some organic carbon will pyrolize during this process and cause an error in the reading. The instrument overcomes this by measuring the change in absorbance of a tuned diode laser that corresponds to the oxidation of this pyrolized carbon during the second stage [75].

\subsubsection{PM Filters}

PM samples were collected onto two different filters depending on the analysis to be performed. Gravimetric measurements were carried out on $47 \mathrm{~mm}$ Pall Teflo filters, while pre-fired $47 \mathrm{~mm}$ Pall Tissuquartz filters were used for EC/OC analyses. These filters meet the US EPA requirements listed in [41] and their specifications are provided in Table 4.4.

Table 4.4: Filter specifications for both gravimetric and EC/OC analysis.

\begin{tabular}{|c|c|c|}
\hline & Teflo Membrane [76] & Tissuquartz [77] \\
\hline Material & PTFE with PMP support & Pure Quartz \\
\hline Nominal Diameter & $47 \mathrm{~mm}$ & $47 \mathrm{~mm}$ \\
\hline Aerosol Retention & $99.99 \%$ & $99.90 \%$ \\
\hline Pore Size & $2 \mu \mathrm{m}$ & $2 \mu \mathrm{m}$ \\
\hline
\end{tabular}




\subsection{Gaseous Sampling}

The following section discusses the four instruments used for gaseous sampling in this investigation. An FTIR provided gaseous speciation, while a standard emissions bench gave regulated emissions concentrations. A small $\mathrm{CO}_{2}$ monitor was used to measure dilution ratios of the two diluters and an AFR sensor provided corrected fuel-air equivalence ratios for the fuel used. Figure 4.1 shows the arrangement of the equipment used.

\subsubsection{Fuel-Air Equivalence Ratio}

The research engine is nominally equipped with a wide-band oxygen sensor that provides the PCM with a reading of the AFR for closed loop control. However, an additional, highly accurate and stand-alone AFR sensor system was employed to gather independent AFR data from the exhaust stream. This was done with an ECM 2400E-1 wide-band oxygen sensor in conjunction with an ECM AFRecorder 2400 to provide real-time AFR data. The main benefit of this system is that the AFRecorder can be configured for different fuel compositions in order to display a corrected AFR reading [78]. The coefficients used in this study are listed in Table 4.5 below; values not given by the US EPA in [79] were found by other means. Ethanol blends not listed were found using a linear fit of the given data (see Appendix A.2.1), while toluene based blends were found using a volumetric balance of constituents (see Appendix A.2.2). For reference on the fuel blends used and their respective nomenclature see Section 4.7.

Table 4.5: Atomic ratios for the different fuel blends.

\begin{tabular}{ccc}
\hline Test Fuel & Atomic Ratio (Normalized) & Source \\
\hline E0 & $\mathrm{CH}_{1.85} \mathrm{O}_{0} \mathrm{~N}_{0}$ & {$[79]$} \\
E10 & $\mathrm{CH}_{1.92} \mathrm{O}_{0.03} \mathrm{~N}_{0}$ & {$[79]$} \\
E30 & $\mathrm{CH}_{2.18} \mathrm{O}_{0.14} \mathrm{~N}_{0}$ & Regression \\
T10 & $\mathrm{CH}_{1.78} \mathrm{O}_{0} \mathrm{~N}_{0}$ & Eq. A.1 \\
T10E10 & $\mathrm{CH}_{1.89} \mathrm{O}_{0.03} \mathrm{~N}_{0}$ & Eq. A.2 \\
\hline
\end{tabular}

\subsubsection{FTIR}

An MKS 2030HS Fourier Transform Infrared Spectroscopy (FTIR) was used to perform gaseous speciation of the exhaust gas in real time. Raw exhaust is drawn from the sample tube through a heated filter that removes exhaust particles from the sample stream. A heated sample line carries the remaining gas phase species to the FTIR analyzer. Both the heated filter and the heated sample line were operated at $191^{\circ} \mathrm{C}$. Data was recorded using the MKS supplied FTIR software at $2 \mathrm{~Hz}$. The software permits the off-line reprocessing of spectral data for different "recipes" - pre-selected lists of compounds for analysis.

The FTIR operates on an infrared light absorption measurement technique, not dissimilar to NonDispersive Infrared emissions analyzers. They differ, however, in the number of gaseous species they are capable of analyzing; where an emission analyzer is typically limited to a single compound (single wavelength), an FTIR can analyze any compound that falls within its spectrum. In this case, the FTIR operates in the mid-infrared region of 2-20 $\mu \mathrm{m}$ and any compound that absorbs infrared radiation in this region can be measured [80]. An interference pattern is passed through the gas sample and the resulting 
light is measured and converted to an absorbance spectrum using Fast Fourier Transform mathematics [80]. Using reference files for pure species, the instrument deconstructs the spectrum based on a preselected batch of species assumed to be in the sample; known as the 'recipe'. The development of the recipe is of utmost importance to the operation of the instrument. Including compounds not present in the sample, or conversely, excluding compounds actually present, will result in erroneous readings. The FTIR recipe used in this investigation is detailed in Section 5.2.1.

\subsubsection{Standard Emissions Bench}

An emissions bench measured raw exhaust concentrations of the standard (regulated) emissions. These include total hydrocarbons (THC), oxides of nitrogen $\left(\mathrm{NO}_{\mathrm{x}}\right)$, carbon dioxide $\left(\mathrm{CO}_{2}\right)$, oxygen $\left(\mathrm{O}_{2}\right)$, and carbon monoxide (CO). Data was logged using the Labview program through the TCP/IP protocol; a network switch connected all four analyzers to the logging computer. Table 4.6 summarizes the analyzers including the ranges used and their respective calibration cylinders. All analyzers were zeroed with emissions grade nitrogen $\left(\mathrm{N}_{2}\right)$. The four analyzers were made by California Analytical Instruments (CAI) and they each conform to US EPA guidelines for measuring their respective species. The same dry air supply used for the aforementioned diluters was also used for the emissions bench (see Section 4.5.3)

The exhaust sample is drawn in through the sample probe at the heated filter head. A filter removes particles from the exhaust stream while maintaining the sample temperature at $180{ }^{\circ} \mathrm{C}$. A heated $3 / 8$ in. Teflon sample line carries the exhaust sample to the emissions bench from the heated filter, where it is teed off to the different analyzers. The two heated analyzers ( $\mathrm{THC}$ and $\mathrm{NO}_{\mathrm{x}}$ ) have internal sample pumps which pull the sample through their respective heated sample lines (each set to $149{ }^{\circ} \mathrm{C}$ ). The two unheated analyzers $\left(\mathrm{CO}_{2} / \mathrm{O}_{2}\right.$ and $\left.\mathrm{CO}\right)$ use an external sample pump which passes the sample through two chillers to remove water (a source of interference in these analyzers). A calibration flow drawer controls the flow of calibration and sample gases to the probe; the exact calibration method is described in Section 5.2.2.

Table 4.6: Standard emissions analyzers, their ranges and calibrations. Calibration cylinders are balanced in $\mathrm{N}_{2}$.

\begin{tabular}{|c|c|c|c|c|c|}
\hline \multicolumn{4}{|c|}{ Emissions Analyzers } & \multicolumn{2}{|c|}{ Calibration Cylinders } \\
\hline Model No. & Analyzer Type & Species & Ranges & Concentration & Composition \\
\hline $\begin{array}{l}\text { CAI } 600 \\
\text { HFID }\end{array}$ & $\begin{array}{l}\text { Heated Flame Ionizing } \\
\text { Detection }\end{array}$ & $\mathrm{THC}^{-\mathrm{C}_{3}}$ & $\begin{array}{l}300 \mathrm{ppm} \\
3000 \mathrm{ppm}\end{array}$ & $\begin{array}{l}203 \mathrm{ppm} \\
2000 \mathrm{ppm}\end{array}$ & $\mathrm{C}_{3} \mathrm{H}_{8} / \mathrm{N}_{2}$ \\
\hline $\begin{array}{l}\text { CAI } 600 \\
\text { HCLD }\end{array}$ & $\begin{array}{l}\text { Heated } \\
\text { Chemiluminescence } \\
\text { Detection }\end{array}$ & $\mathrm{NO}_{\mathrm{x}}$ & $\begin{array}{c}100 \mathrm{ppm} \\
1000 \mathrm{ppm} \\
5000 \mathrm{ppm}\end{array}$ & $\begin{array}{l}89.7 \mathrm{ppm} \\
900 \mathrm{ppm} \\
4063 \mathrm{ppm}\end{array}$ & $\mathrm{NO}_{\mathrm{x}} / \mathrm{N}_{2}$ \\
\hline $\begin{array}{l}\text { CAI } \\
601 P^{*} / 602\end{array}$ & $\begin{array}{l}\text { Non-Dispersive Infrared } \\
\text { and Paramagnetic* }\end{array}$ & $\begin{array}{l}\mathrm{CO}_{2} \\
\mathrm{O}_{2}{ }^{*}\end{array}$ & $\begin{array}{c}14.0 \% \\
1.1 \%\end{array}$ & $\begin{array}{l}13.7 \% \\
0.99 \% \\
\end{array}$ & $\begin{array}{c}\mathrm{CO}_{2} / \mathrm{N}_{2} \\
\mathrm{O}_{2} / \mathrm{N}_{2}\end{array}$ \\
\hline & & $\mathrm{CO}$ & $15000 \mathrm{ppm}$ & $14500 \mathrm{ppm}$ & $\mathrm{CO} / \mathrm{N}_{2}$ \\
\hline
\end{tabular}




\section{THC Analyzer}

The THC analyzer (600 HFID) is a heated flame ionization detector, which is the accepted technique for measuring hydrocarbon constituents of vehicle exhausts according to the US EPA [81]. It operates by detecting the ions formed during the combustion of organic species in a hydrogen flame [8]. These ions are formed proportionately to the hydrocarbon concentration in the sample stream [8]. Effectively, this analyzer counts the reduced carbon atoms in the flame and reports it as an equivalent concentration of hydrocarbons; in this case on a $\mathrm{C}_{3}$ basis. That means that for every three ions detected (or three carbon atoms), one hydrocarbon is reported. Being a heated analyzer, the internal sample components are maintained at $190{ }^{\circ} \mathrm{C}$ to prevent condensation [82].

\section{$\mathrm{NO}_{x}$ Analyzer}

The $\mathrm{NO}_{\mathrm{x}}$ analyzer (600 HCLD) operates on the principle of chemiluminescence, the acceptable method for measuring this species per the US EPA [83]. Incoming NO species are reacted with ozone $\left(\mathrm{O}_{3}\right)$ to form $\mathrm{NO}_{2}$ molecules that are electronically excited [8]. As they decay, they release radiation which is detected by a photomultiplier; the amount of radiation is proportional to the amount of NO in the sample [8]. This instrument also has the capability to convert the $\mathrm{NO}_{2}$ in the exhaust to $\mathrm{NO}$ by decomposition, before being reacted with the ozone [84]. The reported concentration in this configuration, as used in this investigation, is therefore $\mathrm{NO}_{\mathrm{x}}\left(\mathrm{NO}+\mathrm{NO}_{2}\right)$. The internal temperature of components in contact with sample gas are maintained above $68{ }^{\circ} \mathrm{C}[84]$.

\section{$\mathrm{CO}_{2} / \mathrm{O}_{2}$ and $\mathrm{CO}$ Analyzers}

Two unheated analyzers were used for the remaining three gases of the emissions bench: 601P NDIR for $\mathrm{CO}_{2} / \mathrm{O}_{2}$ and 602 NDIR for $\mathrm{CO}$. Both analyzers operate on the principle of non-dispersive infrared analysis, with the $\mathrm{CO}_{2}$ analyzer having an optional paramagnetic analyzer for $\mathrm{O}_{2}$. These are both accepted practices for measuring these species according to the US EPA [85, 86]. The NDIR analyzer operates on the principle of infrared absorption where the radiation absorbed in a sample cell is compared to a reference cell [8]. Radiation not absorbed by the exhaust sample is absorbed by one side of the detector, while the radiation not absorbed by the reference gas is absorbed by the other side [8]. Different amounts of absorption in the two halves causes diaphragm distention from the resulting difference in pressure [8]. A microflow detector measures the induced flow between the two halves, which is converted

to species concentration using the calibration as a reference [87]. As water causes a large intereference in the infrared spectrum for the above compounds, it must be removed from the sample before being analyzed (see Section 4.6.3).

\subsubsection{Mini $\mathrm{CO}_{2}$ Monitor}

A LI-COR LI-840A CO $\mathrm{CO}_{2} / \mathrm{H}_{2} \mathrm{O}$ analyzer $\left(\mathrm{CO}_{2}\right.$ monitor) was used to measure diluted $\mathrm{CO}_{2}$ concentrations to calculate true dilution ratios of the two diluters. The $\mathrm{CO}_{2}$ monitor operates on the principle of NDIR [88], just as the analyzer in the emissions bench does. A small sample pump draws the diluted exhaust stream to the analyzer through $1 / 4$ in. Teflon tubing. The unit incorporates a water sensor that corrects the $\mathrm{CO}_{2}$ reading for water interference [88], so no chiller is necessary. 


\section{7 $\quad$ Test Fuels}

Several different blends of fuel were used in this study, including two different suppliers of commercially available gasoline. The gasoline served as the base for the fuel blends created. All gasoline used was 91 octane pump gasoline from local fuel stations. Fuel blends were created by mixing metered amounts of the different fuels and splash blending them in fuel cans. A note about nomenclature: gasoline is referred to as being E0 (containing $0 \%$ by volume ethanol and $100 \%$ by volume gasoline). The fuel blends used in this investigation listed below are so named after the volumetric percentage of the minor constituent(s).

\subsubsection{Fuel A}

Fuel A was the original supplier of base commercial gasoline used for this project. For earlier work not presented here, no ethanol was observed in the exhaust stream and therefore, it was used as the base gasoline. However, the fuel blend appeared to change to include ethanol prior to the start of the work here, as ethanol exhaust levels similar to previous E10 measurements were being seen. Therefore, data collected using this fuel supplier should not be considered an E0 blend, and data presented herein is clearly identified by the fuel used.

\subsubsection{Fuel B}

As Fuel A appeared to contain ethanol, it was no longer suitable for both the baseline gasoline (E0) and the fuel blends. A different fuel supplier (Fuel B) was found to contain no ethanol in the exhaust stream and was therefore, selected as the new E0 fuel for this investigation. The below fuel blends were created using Fuel B as the base gasoline, unless otherwise stated.

\subsubsection{Fuel Blends}

\section{E10}

A 10\% ethanol-90\% gasoline by volume blend of fuel. Anhydrous ethanol of $99.9 \%$ purity and Fuel B form the two constituents.

\section{E30}

A 30\% ethanol-70\% gasoline by volume blend of fuel. Anhydrous ethanol of $99.9 \%$ purity and Fuel B form the two constituents.

\section{T10}

A $10 \%$ toluene- $90 \%$ gasoline blend of fuel. Reagent grade toluene of $99.5 \%$ purity and Fuel B form the two constituents.

\section{T10E10}

A $10 \%$ toluene- $10 \%$ ethanol- $80 \%$ gasoline by volume blend of fuel. Anhydrous ethanol of $99.9 \%$ purity, reagent grade toluene of $99.5 \%$ purity, and Fuel B form the three constituents. 


\section{Chapter 5}

\section{Experimental Methods}

The following outlines the experimental methods and procedures developed and used in this investigation. Given that the motivation for this study was to expand on and identify sources of variability that were first seen by Mireault in [44], a similar set of engine testing and emissions sampling procedures were used here.

\subsection{Engine Testing}

Two main engine testing conditions were used in this investigation; the transient operation of starting an engine and a steady-state, highway cruise equivalent condition. A pseudo drive cycle was therefore, developed for this work by linking together transient procedures with an intermediate steady-state operation period. This represents a progression to the ultimate goal of performing a simplified drive cycle, similar to other standard drive cycle tests like the FTP-75 and NEDC. Figure 5.1 shows a typical test used in this work. Note that for the sake of consistency, all steady-state data is presented in terms of the start of the entire test (total test time scale in Figure 5.1); the starts use the engine starting as their zero point. The following section details the exact engine procedures performed.

\subsubsection{Pre-Engine Test Set-up}

Preparing the research engine for any of the subsequently discussed engine tests involves first verifying that engine is in good working order. This includes checking the oil level on the dipstick and ensuring that there is sufficient coolant in the expansion tank. Approximately one hour prior to a run, the dynamometer controller is turned on and allowed to warm-up. Cooling water supply to the coolant heat exchanger and fuel cooler is turned on, as is the water supply to the dynamometer. The compressed air to the water valve controller is also opened. While the water is pre-cooling the heat exchangers, if necessary, the battery for the electrical systems of the engine is verified to be fully charged. The drain valve from the PCV filter is closed, and the filter and housing are cleaned of any collected liquid oil. Finally, the fuel tanks are filled with the desired test fuel in preparation for the test.

With the engine physically ready to start, the remaining control systems are readied. Both the throttle control and main Labview programs are opened and started on the logging computer, while the

OBD-II software is opened on the secondary computer. The engine PCM is then enabled on the control panel, followed by the fuel pump. The engine is now effectively in standby and ready to be turned on. 


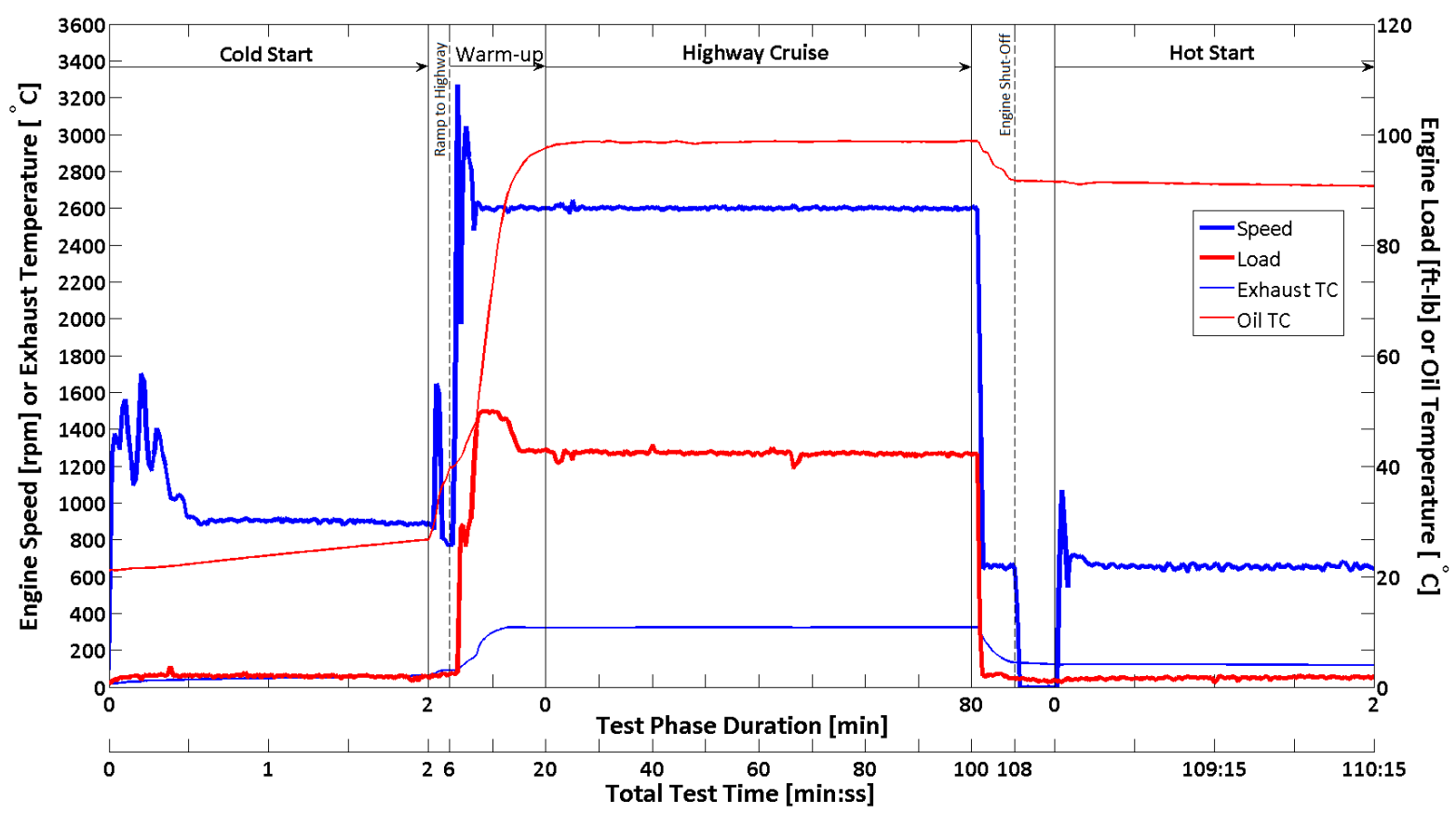

Figure 5.1: Typical engine test procedure; note that time scale (x-axis) varies through the test cycle. "Hot Start" sequence is shown but is optionally performed. Exhaust TC refers to TC4 on Figure 4.1.

\subsubsection{Transient Test Procedures}

Two different engine starts were performed on the basis of the engine temperature condition, those being a cold start and a hot start. The two test conditions differ in their current engine temperature condition and the most recent operation of the engine. For the purposes of this work, the length of an engine start test is considered as two minutes from the initiation of engine cranking - i.e. when the starter begins to spin the engine - and includes a period of engine idling. During the starting procedure and subsequent idle, no load is applied to the engine and the PCM is free to control the desired idle speed with no external throttle input. The following paragraphs describe the general procedures for both a cold and hot start.

\section{Cold Start}

A cold start test begins with the previous run as engine and exhaust preconditioning are important to ensure repeatable starting conditions. Within this framework, for a start to be considered a cold start, a minimum of 12-24 hours of soak time is required since the most recent fully warm, steady-state engine operation. This soak period allows the engine's block, head, oil, and coolant to return to ambient temperatures, which in this case was controlled by the test cell's HVAC system to $22 \pm 2{ }^{\circ} \mathrm{C}$. Following this soak time the coolant heat exchanger is pre-cooled to $20{ }^{\circ} \mathrm{C}$ with city water prior to the test start. Immediately prior to the test starting, the fuel cooler is enabled and the fuel is circulated through the system to pre-cool it to the test temperature of $22{ }^{\circ} \mathrm{C}$. The engine is now ready for a cold start test and the engine start button is pressed and held until the engine starts and data is collected for the remainder of the two minute period. The left-most section of Figure 5.1 shows a typical cold start test. City water flow to the coolant heat exchanger is increased to keep the engine coolant temperature below $30{ }^{\circ} \mathrm{C}$ before the two minutes are up. Table 5.1 contains a list of the important engine parameter set-points 
and tolerances for a cold start.

Table 5.1: Cold start engine parameters and set-points.

\begin{tabular}{lcc}
\hline Parameter & Setting & Tolerance \\
\hline Duration (minutes) & 2 & N/A \\
Engine Speed (rpm) & Idle & N/A \\
Engine Load $(\mathrm{ft}-\mathrm{lb})$ & $\mathrm{N} / \mathrm{A}$ & +2 \\
ECT Start $\left({ }^{\circ} \mathrm{C}\right)$ & 20 & \pm 2 \\
ECT End $\left({ }^{\circ} \mathrm{C}\right)$ & 29 & \pm 2 \\
Fuel Temperature $\left({ }^{\circ} \mathrm{C}\right)$ & 22 & \pm 2 \\
\hline
\end{tabular}

\section{Hot Start}

Like the cold start test, the hot start test is dependent on the current engine and exhaust conditions; however, where the cold start test was performed after an extended soak period, a hot start test is performed from a fully warmed engine state. In this investigation, all hot starts were performed at the end of a 90-100 minute run, unless otherwise noted. The right-most section of Figure 5.1 shows a typical hot start test. After the engine is unloaded from the highway cruise condition, the engine is allowed to idle for several minutes until the exhaust temperature at the end of the sampling tube (T/C 4) reaches $125^{\circ} \mathrm{C}$. During this idling period, the cooling water to the coolant heat exchanger is reduced to maintain a fully warmed coolant temperature of $90{ }^{\circ} \mathrm{C}$. Upon reaching the desired exhaust temperature listed above, the engine is shut down by cutting the ignition switch on the control panel. The engine is restarted after 15 seconds and allowed to idle at the PCM desired engine speed and with no load applied. Just as in the cold start test, data is collected for two minutes from the beginning of engine cranking. Table 5.2 lists the important control parameters during a hot start test sequence. At the end of the hot start test period, the engine is brought up to a steady-state highway cruise condition for a minimum of 10 minutes prior to being cooled and subsequently shut down - this is not shown in Figure 5.1. This is to ensure that a typical engine and exhaust condition is encountered in the proceeding cold start test.

Table 5.2: Hot start engine parameters and set-points.

\begin{tabular}{lcc}
\hline Parameter & Setting & Tolerance \\
\hline Duration (minutes) & 2 & N/A \\
Engine Speed $(\mathrm{rpm})$ & Idle & N/A \\
Engine Load $(\mathrm{ft}-\mathrm{lb})$ & $\mathrm{N} / \mathrm{A}$ & +2 \\
T/C 4 Exhaust Temp Start $\left({ }^{\circ} \mathrm{C}\right)$ & 125 & \pm 2 \\
ECT Start $\left({ }^{\circ} \mathrm{C}\right)$ & 90 & \pm 2 \\
ECT End $\left({ }^{\circ} \mathrm{C}\right)$ & 90 & \pm 2 \\
Fuel Temperature $\left({ }^{\circ} \mathrm{C}\right)$ & 22 & \pm 2 \\
\hline
\end{tabular}




\subsubsection{Steady-State Test Procedures}

The steady-state test procedure is a simulation of a highway cruise condition in a production vehicle. Using vehicle specifications, the road load power requirement for a speed of $100 \mathrm{~km} / \mathrm{h}$ in top gear was calculated. At this condition, the engine experiences a load of $42.7 \mathrm{ft}-\mathrm{lbs}$ at a speed of $2640 \mathrm{rpm}$, though for simplicity the load was rounded down to $42 \mathrm{ft}-\mathrm{lbs}$ and the engine speed down to $2600 \mathrm{rpm}$. Appendix B.1 details this calculation.

\section{Ramp to Highway}

As previously discussed, a throttle pedal simulator was used to programatically control the commanded throttle position in addition to the engine speed control from the dynamometer. For this investigation, a standard ramp to highway cruise from an idle condition was developed. This ramp ensures that the speed and load is smoothly advanced and performed in the same fashion in each and every test. The engine and throttle position (load) ramps are detailed in Table 5.3. The ramp to highway cruise is executed once the two minute cold start test and subsequent four minute idle warm-up is completed - see centre portion of Figure 5.1. During this idle period all engine systems are verified to be in operation and the ECT warms up to approximately $50{ }^{\circ} \mathrm{C}$. The final throttle position in the ramp corresponds to the desired loading at fully warmed conditions; however, because of slight variances in conditions some slight adjustments from this final value may be required.

Table 5.3: Throttle ramp from idle up to highway cruise conditions.

\begin{tabular}{cccc}
\hline Throttle Position (\%) & Engine Speed (rpm) & Execution Start Time (s) & Duration (s) \\
\hline \multirow{2}{*}{7000} & 0.0 & 0 & 1 \\
& 5.0 & 1 & 3 \\
10.0 & 4 & 3 \\
15.0 & 7 & 3 \\
18.0 & 10 & 3 \\
19.0 & 13 & 3 \\
20.0 & 16 & 3 \\
21.0 & 19 & 3 \\
& 21.5 & 22 & 3 \\
& 22.0 & 25 & 4 \\
& 22.5 & 29 & 4 \\
& 22.9 & 33 & 3 \\
\hline
\end{tabular}

\section{Warm-up}

The warm-up phase begins once the engine speed and load have stabilized at their desired set points. During this phase the engine continues to warm-up to fully warm engine coolant and engine oil conditions; see Table 5.4 for a list of steady-state temperatures and parameters for reference. As the ECT approaches $90{ }^{\circ} \mathrm{C}$ the cooling water flow through the coolant heat exchanger is increased until stabilization of this temperature occurs. The engine oil temperature is the last parameter to achieve stabilization and under 
typical conditions reaches its set-point approximately 10-15 minutes after commencing highway cruiseor approximately 20 minutes after cold start (ACS).

\section{Steady Conditions}

Table 5.4 lists the steady-state conditions at the simulated highway cruise engine operation. Emissions data from steady-state conditions was recorded from 20 minutes ACS until the end of the run, including any subsequent hot start tests. A note to the reader; a couple of hot soak tests, where the engine was started from a non-cold soak condition, were performed in this investigation. Therefore, measurements from these tests were recorded earlier than 20 minutes ACS, as temperature stabilization occurred much sooner.

Table 5.4: Highway cruise parameters and set-points.

\begin{tabular}{lccc}
\hline Parameter & Cooler Enabled & Set-point & Tolerance \\
\hline ECT $\left({ }^{\circ} \mathrm{C}\right)$ & $\boldsymbol{J}$ & 90 & \pm 2 \\
Fuel Temperature $\left({ }^{\circ} \mathrm{C}\right)$ & $\boldsymbol{x}$ & 22 & \pm 2 \\
& $\boldsymbol{J}$ & 99 & \pm 2 \\
Oil Temperature $\left({ }^{\circ} \mathrm{C}\right)$ & $\boldsymbol{X}$ & 105 & \pm 2 \\
& $\mathrm{~N} / \mathrm{A}$ & 22 & \pm 2 \\
Intake Air Temperature $\left({ }^{\circ} \mathrm{C}\right)$ & $\mathrm{N} / \mathrm{A}$ & 320 & \pm 5 \\
TC4 Exhaust Temperature $\left({ }^{\circ} \mathrm{C}\right)$ & $\mathrm{N} / \mathrm{A}$ & 2600 & \pm 30 \\
Engine Speed $(\mathrm{rpm})$ & $\mathrm{N} / \mathrm{A}$ & 42 & \pm 1 \\
Load $(\mathrm{ft}-\mathrm{lb})$ & & & \\
\hline
\end{tabular}

\section{Cool-down and shut down}

At the end of the steady-state test condition - typically 90-100 minutes ACS - the engine is smoothly brought down to the idle condition and unloaded. If a hot start test is not being performed, then the engine is allowed to idle for several minutes while the engine continues to cool gradually. With the ECT at $60{ }^{\circ} \mathrm{C}$ and the oil temperature below $80{ }^{\circ} \mathrm{C}$ the engine is shut off and the test is considered complete. Note that this shut-down procedure is omitted from the simplified drive-cycle Figure in 5.1.

\subsubsection{Fuel Change-over}

A fuel change-over procedure was followed to limit fuel blend cross contamination and to allow for $n$ cold start tests from $n$ steady-state tests. The fuel tanks are first fully drained of their current fuel using a hand siphon. A small amount of the subsequent fuel blend (approximately two litres) is then poured into the fuel tank. The engine is started and brought up to a steady-state condition as described in Section 5.1.3 and run for 20 minutes, or until the engine is considered thermally at steady-state. The engine is then cooled and shut down, with the remaining fuel drained out of the fuel tank. Freshly blended fuel is then filled into the tank(s) and after the 12-24 hour soak period the engine is ready to start a new test cycle on the new fuel blend. 


\subsection{Gaseous Emissions Sampling}

The following section outlines the collection of gaseous emissions data using the instruments described in Chapter 4.

\subsubsection{FTIR}

An FTIR was used to provide a speciation of the exhaust constituents. The recipe of gases for the FTIR used in this investigation was a variation on the one presented by Mireault [44]. Two compounds, ethane and benzene, were reintroduced into the recipe as they were found in sufficient quantities in the exhaust gases. Ethanol - marked by an asterisk in the method list below - was only included in the recipe if it was expected or known to be a constituent in the fuel. Note that this can be, and was, performed retroactively through the reprocessing function built into the MKS software used.

Table 5.5: Compounds used in the FTIR method.

\begin{tabular}{llll}
\hline Nitric Oxide $(\mathrm{NO})$ & Carbon Monoxide $(\mathrm{CO})$ & Carbon Dioxide $\left(\mathrm{CO}_{2}\right)$ & Water $\left(\mathrm{H}_{2} \mathrm{O}\right)$ \\
Nitrogen Dioxide $\left(\mathrm{NO}_{2}\right)$ & Methane $\left(\mathrm{CH}_{4}\right)$ & Ethane $\left(\mathrm{C}_{2} \mathrm{H}_{6}\right)$ & Pentane $\left(\mathrm{C}_{5} \mathrm{H}_{12}\right)$ \\
Nitrous Oxide $\left(\mathrm{N}_{2} \mathrm{O}\right)$ & Ethylene $\left(\mathrm{C}_{2} \mathrm{H}_{4}\right)$ & Propylene $\left(\mathrm{C}_{3} \mathrm{H}_{6}\right)$ & Isobutylene $\left(\mathrm{C}_{4} \mathrm{H}_{8}\right)$ \\
Acetylene $\left(\mathrm{C}_{2} \mathrm{H}_{2}\right)$ & 1,3 -Butadiene $\left(\mathrm{C}_{4} \mathrm{H}_{6}\right)$ & Benzene $\left(\mathrm{C}_{6} \mathrm{H}_{6}\right)$ & Toluene $\left(\mathrm{C}_{7} \mathrm{H}_{8}\right)$ \\
Formaldehyde $\left(\mathrm{CH}_{2} \mathrm{O}\right)$ & Acetaldehyde $\left(\mathrm{CH}_{3} \mathrm{CHO}\right)$ & Methanol $\left(\mathrm{CH}_{3} \mathrm{OH}\right)$ & Ethanol* $\left(\mathrm{C}_{2} \mathrm{H}_{5} \mathrm{OH}\right)$ \\
\hline
\end{tabular}

\section{Warm-up}

Operating the FTIR begins by filling up the dewar with liquid $\mathrm{N}_{2}$. The vent on the dewar is opened to purge the escaping air from the optics box. During this period the power to the auxiliary heaters, such as the heated filter, heated lines, and the heated pump blankets, are turned on and allowed to heat up to their set-point of $191^{\circ} \mathrm{C}$.

\section{Systems Check and Background}

With the FTIR fully warmed and its dewar filled with liquid $\mathrm{N}_{2}$ the systems check portion of the startup procedure is done. Several diagnostic tests are performed to verify that the instrument is within its operational tolerances. This is done prior to every run and ensures measurement confidence. With the systems check finished, the above mentioned recipe is loaded and the file directories selected. A zero gas background is taken by flowing emissions grade $\mathrm{N}_{2}$ through the instrument for a couple of minutes. The background represents the zero spectrum from which the measured exhaust spectrum will be compared to in determining the species and their concentrations. This is taken before the start of each run and ensures consistency for run-to-run comparisons. With the background completed the instrument is ready to measure engine exhaust and is left in standby mode until measurement is desired.

\section{Operation}

To measure the exhaust gas constituents, the FTIR is set to sample by turning on the integrated sample pumps and toggling off the purge gas to the instrument. It is important to carefully monitor the temperature of the instrument and auxiliary heaters as they can either drop or climb out of specification, 
depending on the temperature of the raw exhaust. For this reason, the FTIR was only operated at steady-state engine operation with a sufficiently hot exhaust temperature.

\section{Shut-down}

The shut down procedure involves shutting off the sample pumps and toggling on the purge gas supply to the instrument. As the files are automatically saved, no further user intervention is required beyond turning off the auxiliary heaters.

\subsubsection{Emissions Bench}

The following section details the operation of the emissions bench. See Section 4.6.3 for a list of analyzers and calibration cylinders used. On-line oxygen measurement corrections were performed in real time in the Labview environment-Appendix B.2 details the correction formulas used and the analyzer based correction factors.

\section{Warm-up}

Ample analyzer warm up time is required in order to thermally stabilize the heated analyzers (HFID and HCLD). The heated sample filter and sample lines are typically left on between runs as they can require several hours to heat to their set-points. First the analyzers are awaken from their standby state and the sample pump for the NDIR $\left(\mathrm{CO}_{2} / \mathrm{O}_{2}\right.$ and $\left.\mathrm{CO}\right)$ analyzers is turned on. The dry air supply and heli fuel (40\% Hydrogen, $60 \%$ Helium) cylinder are then opened and both the HFID and HCLD analyzers are ready to be enabled. The burner in the HFID is subsequently ignited, and the internal sample pump is enabled once ignition is achieved; the HCLD requires only its internal sample pump to be enabled to begin sampling. The remaining two NDIR analyzers are also set to sample and the entire emissions bench is left to warm-up, while sampling room air, for another 30-40 minutes.

\section{Calibration}

With the emissions bench fully warmed, the calibration procedure can commence; a list of calibration cylinders is included in Table 4.6. First the zero and calibration gas lines are connected to back panel of the emissions bench, and their regulators are opened and set to approximately 10 psig. The emissions bench incorporates a flow drawer which allows quick selection between the gases. With the zero gas selected on the flow drawer, the toggle valve at the heated filter is switched from room air to the calibration line as the calibration is done at the probe. The zero gas is now allowed to stabilize and flow through the four analyzers for at least four minutes before they are zeroed. The zero cylinder is then closed and the flow drawer is switched to the next calibration gas once the pressure in the line has purged. Each proceeding span is calibrated in the same manner as above until all the required ranges have been spanned.

\section{Operation}

After the calibration is completed the emissions bench is effectively ready to measure exhaust gas. The TCP/IP network switch is connected to the logging computer and the toggle valve at the probe head is set to sample from the exhaust sample tube. In this configuration the exhaust gas is now being 
pulled through the emissions bench and is being analyzed. Values are automatically recorded if the main Labview program (see Section 4.4.1) is running. The HCLD $\left(\mathrm{NO}_{\mathrm{x}}\right)$ analyzer is placed in auto range mode, where the analyzer automatically switches the measurement range based on the measured value; the other analyzers are left in their respective ranges listed in Table 4.6. Periodic checks on the analyzers are performed during a run to ensure no errors have occurred and to verify that the drains from the chillers are removing the condensed water vapour.

\section{Shut-down}

The shut down procedure starts with switching the toggle valve at the heated filter back to room air. This is done to purge the analyzers from the exhaust sample and water moisture build up that comes from sampling engine emissions. For this reason, the analyzers are run for a minimum of 30 minutes on room air after a run before they can be placed on standby. After the 30 minute period has elapsed the heli fuel supply is shut off and the HFID analyzer is run until the flame extinguishes from a lack of fuel pressure. While the flame extinguishes, the other analyzers are placed on standby and the external sample pump for the NDIRs is shut off. The HFID is also placed in standby mode once its flame extinguishes and its internal sample pump automatically turns off.

\subsubsection{Mini $\mathrm{CO}_{2}$ Monitor}

$\mathrm{CO}_{2}$ was used a tracer gas in this investigation to calculate the true dilution ratio of the two diluters used. The mini $\mathrm{CO}_{2}$ Monitor was used to measure the diluted $\mathrm{CO}_{2}$ concentrations to back calculate the true dilution ratio - see Appendix B.3 for a derivation of the dilution ratio calculation. Operation of the $\mathrm{CO}_{2}$ monitor is straightforward. The unit and its external sample pump are first powered up and allowed to warm-up for 30 minutes prior to a run while sampling room air. Two minutes prior to run start, the LiCor supplied logging software is enabled and set to record, and the $\mathrm{CO}_{2}$ monitor is set to sample diluted EEPS exhaust. This two minute period serves as the baseline $\mathrm{CO}_{2}$ concentration of the dilution air and is used in the off-line calculation of the dilution ratio. During the engine testing the $\mathrm{CO}_{2}$ monitor is switched between sampling the TSI and the Dekati diluter as necessary. Note that both diluters use the same dry air supply so that the measured baseline $\mathrm{CO}_{2}$ concentration prior to run start is applicable to both dilution ratio calculations. At the end of the run the software is shut down, followed by the unit and the sample pump.

\subsection{Particulate Matter Emissions Sampling}

The following will describe the procedures used for the particulate matter sampling equipment, including analyzers and diluters.

\subsubsection{Engine Exhaust Particle Sizer (EEPS)}

The EEPS was used in this investigation to provide real-time particle size distributions and total number (PN) concentrations. Conductive silicone tubing, which limits particle losses, was used to connect the EEPS to the rotating disk thermodiluter. This line length was kept as short as possible to prevent unwanted particle losses. Periodic column and electrode cleaning was performed as needed during this investigation, per the EEPS manual [72]. 


\section{Standby}

When not drawing on engine exhaust, the EEPS is left sampling HEPA filtered room air, which ensures that the column is cleaned of any lingering particles between tests. Leaving the EEPS on and drawing in filtered room air permits less frequent maintenance of the column and furthermore, ensures that the EEPS is always ready for exhaust sampling.

\section{Sampling}

To begin sampling, the EEPS logging software is opened and set to record on the logging computer. This software only permits up to 1.5 hours of continuous logging; a subsequent file is automatically saved after a minimum of one minute stoppage between them. For stability purposes the file was set to record one hour and 28 minutes of data with a two minute pause between. With the logging software recording the EEPS is now connected to the outlet of the rotating disk thermodiluter and begins to sample the diluted exhaust. During sampling, the $\mathrm{CO}_{2}$ monitor is connected to the EEPS exhaust port and measures the diluted $\mathrm{CO}_{2}$ concentration. At the end of the run, the exhaust line from the diluter is disconnected and the HEPA filter is reconnected to the inlet of the EEPS.

\subsubsection{Rotating Disk Thermodiluter}

As previously presented, the rotating disk thermodiluter was used to dilute the raw exhaust for measurement in the EEPS. Both the DR and DT set-points are listed in Table 5.6, which was used regardless of the engine test being performed. These set-points are the same ones used in previous work by Mireault [44], and were carried over to provide the best comparison of data.

Table 5.6: Diluter box settings.

\begin{tabular}{lc}
\hline Parameter & Set-point \\
\hline Primary Dilution Temperature $\left({ }^{\circ} \mathrm{C}\right)$ & 80 \\
Primary Dilution Factor $(\%)$ & 80 \\
Secondary Dilution Factor $(\mathrm{V})$ & 6.3 \\
Thermal Conditioner $\left({ }^{\circ} \mathrm{C}\right)$ & 300 \\
Final Dilution Ratio (nominal) & 122 \\
\hline
\end{tabular}

\section{Warm-up}

Prior to starting the rotating disk thermodiluter, the diluter head is disassembled and any collected water is drained out. This is done to prevent issues of hydrolock which can occur when too much water is present. With the diluter head reassembled the diluter box is turned on and the set-points listed in Table 5.6 are set and the diluter is left to heat-up. Once the temperature and dilution settings are stable (all green indicator lights on the diluter box) the dry air supply is slowly opened. It is important that this is done in a smooth fashion to prevent unwanted pressure pulses from propagating through the dilution system and potentially damaging components. A flow meter on the vent portion of the dry air line is used as a guide for how much air is required. When the flow meter measurement reverses direction, an excess of dry air is available as is desired. An excess air of a $2.5 \mathrm{lpm}$ was used in this 
investigation to ensure that there was always a supply of air for the unit. Once the dry air supply flow has stabilized, the rotating disk thermodiluter is ready to be connected to the EEPS. A small cooling fan directed at the diluter head is turned on prior to run start. This carries over from previous work on this apparatus, which found that the hot engine exhaust in close proximity to the diluter head was causing it to drift in temperature [44]. The fan ensures that dilution temperature remains at the desired set-point.

\section{Sampling}

During sampling, care is required to monitor the dilution settings and verify the diluter remains in desired operation. Small adjustments to the diluter box settings may be required to achieve the desired dilution as the test progresses.

\section{Shut-down}

At the end of the run, the shut down procedure for the diluter box first involves turning up the primary dilution temperature to $150{ }^{\circ} \mathrm{C}$. This is done to evaporate any condensed liquid water that may have collected in the diluter head during engine operation. The diluter box is also disconnected from the EEPS at this time. After approximately 20-30 minutes the diluter head is considered sufficiently dry. The dry air supply is now slowly closed off to the diluter box, much in the same way as it was opened in the warm-up procedure. With the dry air supply closed, the diluter box is turned off and the procedure is complete.

\subsubsection{Filter Cart}

The operation of the filter collection cart used in this work is detailed here. Previous work at the ERDL [70, 71] used a sample flow rate of $20 \mathrm{lpm}$, which was continued in Mireault's work on the GDI [44]. However, the relevant US EPA procedure for PM filter collection states that the filter face velocity should be maintained at or below $100 \mathrm{~cm} / \mathrm{s}$ [41]. At $20 \mathrm{lpm}$, the calculated face velocity is $30 \mathrm{~cm} / \mathrm{s}$, so for this investigation the sample flow rate was increased to achieve the desired filter face velocities. Unfortunately, the maximum rated flow for the controllers is $35 \mathrm{lpm}$, which yields a filter face velocity of $51 \mathrm{~cm} / \mathrm{s}$; short of the desired $100 \mathrm{~cm} / \mathrm{s}$. Nevertheless this represents an improvement over previous work and so for this investigation, the filter collection was performed at $33 \mathrm{lpm}$ for a face velocity just under $50 \mathrm{~cm} / \mathrm{s}$.

The Hot Pocket heater on the cyclone was set to $55^{\circ} \mathrm{C}$ based on experimentation results. This heater temperature was found to underachieve the desired $47 \pm 5^{\circ} \mathrm{C}$ post filter temperature, as stipulated by the US EPA in [73]. However, the physical arrangement of the filter cart has large metallic fittings (large pipe elbow) that act as heat sinks for the flow. Therefore, the measured temperature at the location of thermocouple is likely lower than the temperature immediately following the filter, and so the above temperature set-point was deemed to be appropriate.

The addition of the bypass line (described in Section 4.5.5) requires careful monitoring of the bypass filter on a run-to-run basis. As the filter becomes more and more loaded, the pressure drop through the system increases and the pumps lose their ability to draw sample at the desired flow rate. As such, this filter was replaced as needed during this investigation. 
Table 5.7: Filter cart settings.

\begin{tabular}{lcc}
\hline Parameter & Set-point & Tolerance \\
\hline Flow Controllers $(\mathrm{lpm})$ & 33.0 & \pm 0.5 \\
Hot Pocket Heater $\left({ }^{\circ} \mathrm{C}\right)$ & 55 & \pm 2 \\
\hline
\end{tabular}

\section{Warm-up/Calibration}

Operation of the filter cart begins with an appropriate amount of warm-up time for the mass flow controllers, including their calibration. The sample inlet quick-connect fitting is first disconnected from the Dekati diluter. The power to the filter cart is then turned on and the mass flow controllers are allowed to warm-up and stabilize while set to $0 \mathrm{lpm}$. After approximately 30-40 minutes of warm-up time the flow controllers are set to $9 \mathrm{lpm}$ each and the sample pumps are turned on. The pumps are subsequently turned off after five minutes and the flow controllers are re-set to $0 \mathrm{lpm}$ and zeroed.

With the calibration complete, the filter cart is ready to be set-up for sampling. First, the sample inlet quick-connect is reconnected to the Dekati diluter. The Hot Pocket heater is then turned on and set to the desired temperature of $55{ }^{\circ} \mathrm{C}$. The flow controllers are subsequently set to the sampling flow rate of $33 \mathrm{lpm}$ and the sample pumps are re-started. At this point the filter cart is ready for sampling, assuming the Dekati diluter is also ready (see Section 5.3.4).

\section{Filter Collection}

The filter collection procedure involves first inserting a dummy filter in the filter holder(s) and setting the solenoid valve to sample. A dummy filter is simply a filter that is not to be used for any data collection, and it is used here to warm-up the filter holder for the subsequent actual filter collection. The filter holders are large masses of stainless steel, and therefore, have quite a bit of thermal inertia. This warm-up period is done to prevent unnecessary cooling of the filter holder, and thus the filter itself, during sampling. Approximately 5-10 minutes before collecting the sample was found to be sufficient time to stabilize the post filter temperature.

With the filter holder warmed up, the actual filter cassettes (i.e. the ones holding the filters for analysis) are loaded into the sampling train. The samples are drawn through the filters for the desired time based on the test being performed (see Table 5.8). Immediately following the collection period, the filter cassettes are removed from the holders and placed in sealed containers to prevent exposure to ambient dust and particles. Filters for EC/OC processing are promptly transferred to petri-slides and placed in a cooler to prevent evaporative losses.

Table 5.8: Filter collection durations.

\begin{tabular}{|c|c|c|c|c|}
\hline \multirow[b]{2}{*}{ Engine Test Mode } & \multirow[b]{2}{*}{ Collection Start (min) } & \multicolumn{2}{|c|}{ Duration (min) } & \multirow{2}{*}{$\begin{array}{c}\text { Post Filter } \\
\text { Temperature }\left({ }^{\circ} \mathrm{C}\right)\end{array}$} \\
\hline & & Gravimetric & $\mathrm{EC} / \mathrm{OC}$ & \\
\hline Cold Start & On Engine Crank & $\mathrm{N} / \mathrm{A}$ & 2 & $30 \pm 2$ \\
\hline Hot Start & On Engine Crank & $\mathrm{N} / \mathrm{A}$ & 2 & $30 \pm 2$ \\
\hline Highway Cruise & Variable; $30,60,80$, or 90 ACS & 10,20 , or 40 & 10 & $35 \pm 2$ \\
\hline
\end{tabular}




\section{Shut-down}

Shutting down the filter cart involves first disabling the Hot Pocket heater. The vacuum pumps are then turned off, followed by setting the flow controllers back down to $0 \mathrm{lpm}$. At this point the filter cart is fully shut-down and its electrical power can be turned off.

\subsubsection{Dekati Diluter}

A Dekati ejector diluter was used in this study to dilute exhaust samples for filter collection. The Dekati is a pressure sensitive instrument so a minimum of two vent lines were required to reduce back pressure on the instrument. For this work the medium flow condition was required because of the increase in the filter cart sampling flow rate to $33 \mathrm{lpm}$ (66 lpm total); see Section 4.5.5. Carrying over from the experiences of Mireault's work on the GDI, a nominal DR of 10:1 - the lowest at this flow setting - was used. From experience, the actual DR was found to deviate from the nominal and so it was measured during filter collection for accurate post-measurement correction. The DT was also found to vary depending on the test being performed. Table 5.9 lists the set-points and typical dilution conditions encountered during this investigation. Returning to the literature, previous studies mentioned in Chapter 2 have used similar DR and DT set-points for PM measurement.

Table 5.9: Dekati diluter DR and DT set-points and typical conditions.

\begin{tabular}{lcccccc}
\hline & \multicolumn{2}{c}{ Typical Conditions } & & \multicolumn{2}{c}{ Set-points } \\
\cline { 2 - 3 } Engine Test & Actual DR & Actual DT $\left({ }^{\circ} \mathrm{C}\right)$ & & DR & Probe Heater $\left({ }^{\circ} \mathrm{C}\right)$ & Primary Air Heater $\left({ }^{\circ} \mathrm{C}\right)$ \\
\hline Cold Start & $13: 1$ & 50 & & & & 250 \\
Hot Start & $14: 1$ & 65 & & $10: 1$ & 125 & \\
Highway Cruise & $14: 1-18: 1$ & $70-75$ & & & & \\
\hline
\end{tabular}

\section{Warm-up}

The Dekati diluter undergoes a start-up procedure prior to the start of a test. Assuming that the filter cart is appropriately ready, and that the dry dilution air supply is opened, the Dekati is first started and its control software enabled. The medium flow rate setting is selected on the software along with solenoid valves " 2 " and " 3 " to give the lowest dilution ratio. With the dilution air now flowing, the execution of the probe heater and dilution air heater ramps are performed. The temperature set points are first set to lower than desired temperatures, and subsequently raised smoothly to their final values; Table 5.10 lists the two ramps used here. Care is required to ensure that neither of the two heaters exceeds their rated temperature during the warm-up period; exceeding the temperature causes the heaters to disable and the entire process must start anew.

Table 5.10: Dekati diluter heater ramps.

\begin{tabular}{ll}
\hline Probe Heater $\left({ }^{\circ} \mathrm{C}\right)$ & $75 \rightarrow 100 \rightarrow 125$ \\
Primary Dilution Air Heater $\left({ }^{\circ} \mathrm{C}\right)$ & $75 \rightarrow 125 \rightarrow 175 \rightarrow 225 \rightarrow 250$ \\
\hline
\end{tabular}




\section{Operation}

The Dekati diluter is left on and running through the duration of the run, as the filter collection occurs on an ongoing basis. During filter collection the actual DR is measured using the previously discussed $\mathrm{CO}_{2}$ monitor. A five minute average of the dilution ratio was used in correcting the mass concentrations collected on filters during this work.

\section{Shut-down}

When the run is over, or if the run is not over but no further filters are to be collected, the Dekati may be shut-down. Shut-down begins with first setting the heaters back to $0{ }^{\circ} \mathrm{C}$ and allowing them to begin to cool down. Once the cooling of the heaters is established, the control software is closed and the unit's power switch is turned off.

\subsection{Off-line Filter Analysis}

Two different off-line filter analyses were performed on PM laden filters: gravimetric (mass) and compositional (EC/OC). Filters were collected using the filter cart and Dekati diluter, with the collection procedure detailed in the immediately preceding sections.

\subsubsection{Gravimetric Filter Analysis}

Gravimetric filter analysis was performed in a Class 100 clean room environment, which is kept at a constant temperature and relative humidity listed in Section 4.5.6. The procedures outlined below derive from the US EPA standard operating procedure for PM gravimetric analysis [74]. Filters were rejected from use based on physical defects such as pinholes, thinned areas, de-laminated PMP rings, and visible dirt/particles.

Prior to entering the clean room, appropriate Class 100 clean room attire (full body garment suit, hood, and foot covers) are donned. Any equipment or filter media being brought into the clean room is cleaned with appropriate wet wipes that are lint free and moistened with a $6 \%$ isopropyl alcohol $/ 94 \%$ deionized water mix. Upon entering the clean room, an antistatic wrist band is attached to the user's arm and all work surfaces are wiped down with a wet wipe. The logging computer and microbalance scale are turned on. While the scale performs internal calibrations, the samples to be weighed are prepared in the staging area and transferred over to the weighing bench. Upon weighing completion, the balance and computer are placed into standby and any waste is removed upon exiting the clean room.

\section{Filter Equilibration}

New filter batches are brought into the clean room approximately two weeks prior to planned use for equilibration purposes. After this period, a number of filters (more than the required for the planned test set) are removed and placed in petri-slides. The filters are weighed at 24 hour intervals until their replicate weights stabilize and vary by less than $5 \mu \mathrm{g}$. The exact weighing procedure is detailed below. Filters that have met this criterion are considered ready for final pre-sample weighing. Note the final filter equilibration weight may be used in lieu of a final pre-sample weight if it cannot be performed. 


\section{Pre-Sample Weighing}

The pre-sample weighing procedure begins several hours prior to the planned engine run. The filter to be weighed is placed in the microbalance chamber and the electrostatic neutraliser is turned on. A count-down timer counts until two minutes have elapsed, at which point the chamber door is closed and count-down timer is reset to two more minutes. At the end of this two minute period the filter weight is considered stable and the first reading is recorded in the logging spreadsheet. Note that the operating procedure requires that the weight not change for 20 seconds to be considered stable [74]. However, a two minute stabilization period is employed here to ensure consistency between weighing time as this stabilization length was found to vary in previous experiences. This idea is likewise applied to the two minute electrostatic neutralising period. The now weighed filter is placed in its filter cassette and left in the sealed transport container until just before the collection period begins.

\section{Post-Sample Weighing}

After collecting the sample(s), the Tupperware container with the PM laden gravimetric filters is returned to the clean room. Once in the clean room the samples are returned to their pertri-slides and allowed to equilibrate for 24 hours prior to weighing [74]. After acclimating for 24 hours, the filters are weighed in the same manner as described above and returned to their petri-slides. Follow-up weighings are performed until the weight varies by less than $5 \mu \mathrm{g}$. The final weight of the sequence is recorded as the final sample weight and the filters are archived in their petri-slides for potential future use.

\subsubsection{Filter Composition Analysis}

EC/OC analysis was performed on PM laden Tissuquartz filters. These filters were collected in the manner described in Section 5.3.3. Filters were pre-fired at an off-site facility by another student prior to this work and kept sealed in a freezer at the ERDL. The operation of pre-firing involves baking the quartz filters at high temperatures (exceeding $800{ }^{\circ} \mathrm{C}$ ) for several hours to remove any carbon particles or compounds that may be on the filters. Keeping the filters sealed and in a freezer after the pre-firing is done to prevent adsorption of gaseous compounds (VOCs) that are present in ambient air prior to exposing the filters to engine exhaust.

The EC/OC analysis was performed at the University of Toronto Southern Ontario Centre for Atmospheric Aerosol Research (SOCAAR). The majority of the filters were analyzed by another student, Naomi Zimmerman, with the post-processing work also performed by her ${ }^{1}$. The EC/OC instrument only accepts filter samples that are much smaller than the size of an entire filter; $1.37 \mathrm{~cm}^{2}$ vs. 17.3 $\mathrm{cm}^{2}$. Therefore, each filter had three punches removed and analyzed by the instrument, which is an improvement over a single measurement.

\section{Pre-Sample Preparation}

Preparation begins by removing the pre-fired filters from the freezer and transferring them to the clean room in order to be placed in their filter cassettes. Once in the clean room the filters are carefully removed from their aluminium foil carrier and placed in their respective cassettes. Any aluminium foil flakes on the filters are removed - if the foils cannot be successfully removed, the filter is rejected and a new one

$\overline{{ }^{1} \text { Data has been reported here with her permission. }}$ 
is removed from the batch. The filter cassettes are then placed in a sealed container and returned to the freezer along with the package of unused pre-fired filters. The filter cassettes are kept in this container in the freezer until a couple of minutes from their planned collection period to limit their exposure to ambient air. Following sample collection, the PM laden filters are immediately transferred to their petri-slides and placed on ice packs in a cooler. Filters are then transferred promptly to SOCAAR for immediate analysis (within two hours of collection). This is done to limit the evaporation of condensed volatile species from the filter by keeping its temperature low and limiting the time before analysis.

\section{Analysis Method}

Analysis first begins by removing the small sample punch from the larger filter and placing it in the instrument. The rest of the filter material is placed in the cooler for the remaining two punches. The analyzer is then sealed up and the thermal method is loaded into the control software and set to execute. The thermal method used in this EC/OC filter analysis, which is the same used in previous work [44], is given in Figure 5.2. The thermal program amounts to a custom temperature ramp for the analyzer oven, and a control scheme for the different gases injected. The program was developed specifically for this GDI engine's exhaust, as the previously developed methods for diesel exhaust were found to not appropriately analyze the PM generated from a GDI engine [44]. Once the program has fully executed, the sample present in the analyzer is removed and the process is repeated for the remaining two punches for the filter, and likewise, for any remaining filters. The results file produced at the end of the analysis is ready for post-processing work.

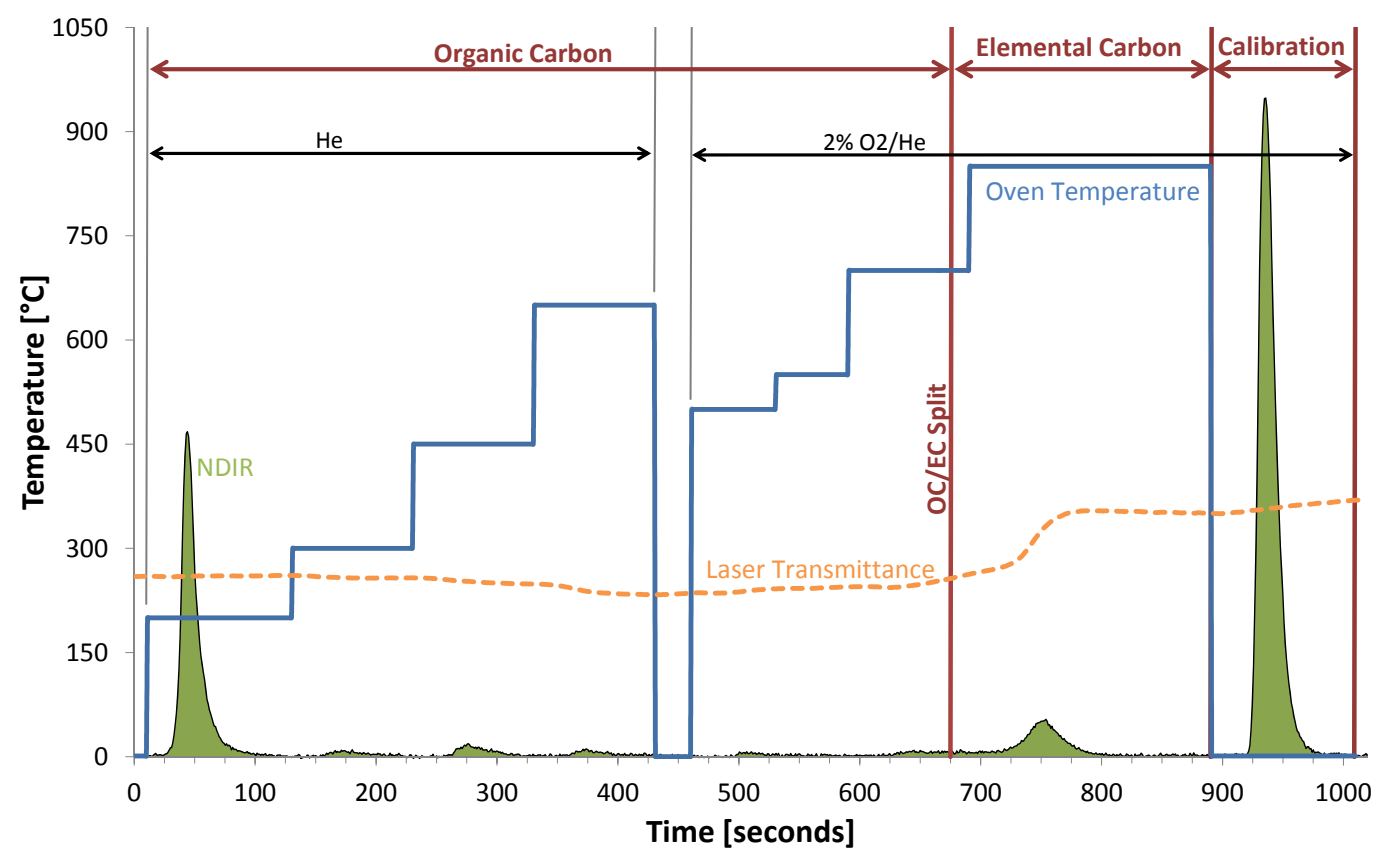

Figure 5.2: GDI EC/OC thermal program with typical NDIR and Laser results.

\section{Post-Processing Work}

With the filter punches analyzed, some post-processing work is required to correct the calculated organic and elemental carbon concentrations given by the instrument. As discussed in Section 4.5.7, some of the 
organic carbon will char/pyrolize when heated under the organic portion of the method. This will show up as elemental carbon and drive the EC/OC ratio upward. The instrument decides on an appropriate split point using internal logic; however, this usually still requires some user manipulation to capture the charred/pyrolized carbon entirely. Therefore, the post-processing work done here is to move the split point to a more suitable location dictated by the NDIR results. This is done using the analysis software and when satisfactorily adjusted automatically updates the results file for the corrected split point. 


\section{Chapter 6}

\section{Steady-State Results}

The steady-state, highway cruise results are presented in this chapter. The first section discusses the sources of variability from a systematic perspective, including hypothesized engine sources and sampling artifacts. The second major section introduces the influence of fuel composition as it pertains to PM emission variability, while the final portion of this chapter analyzes the influence of fuel composition on a more fundamental level, insofar as providing an explanation for observed trends. A summary is provided at the end of this chapter to provide a synthesis of these results. Appendix C provides a repository of supplementary notes for this chapter. Here the reader will find elaborations to discussions and auxiliary plots that would otherwise impede the overall theme of this chapter, but still provide context.

The results presented in this and the following chapter are from a variety of emissions measurement equipment, which are listed in Chapter 4. Unless otherwise noted, PM emissions (number concentrations, size distributions, mass, and composition) have been corrected for the measured dilution ratio. Likewise, $\mathrm{O}_{2}$ measurements from the emissions bench are corrected for compound interference. Gaseous emissions are duly reported dry or wet as appropriate for the species in question.

\section{Test Matrix}

The results of this work are presented here in a quasi chronological order. It is important that the reader understand that this is done to convey the progression and order-sensitive nature of the investigation performed. A test matrix is provided which organizes the tests performed for this work based on their test grouping - see Table 6.1. In reading these results, references to this test matrix are made for the purposes of orienting the reader in the work. The test matrix is broken down first by the test group (i.e. the hypothesis/fuel being tested) and the month(s) in which those tests occurred. Note that colour coordination between this test matrix is maintained with the subsequently presented figures to aid the reader, where relevant. Test details are given regarding the types of tests performed and more importantly the base fuel and fuel blend used. A list of major instrumentation used for each test is also given, along with a test-by-test listing of the major modifications performed throughout this investigation. This provides the reader with a full break-down of which modifications provided in Chapter 4 were enabled for each test, and offers better insight to comparisons made here. 


\begin{tabular}{|c|c|c|c|c|c|c|c|c|c|c|c|c|}
\hline 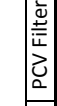 & 2 & $\stackrel{0}{2}$ & $\stackrel{0}{2}$ & & $\stackrel{0}{2}$ & $\stackrel{\check{\nu}}{\rightleftharpoons}$ & $\stackrel{\square}{\rightleftharpoons}$ & $\stackrel{\square}{\rightleftharpoons}$ & $\stackrel{\check{\varpi}}{=}$ & 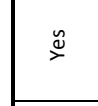 & $\stackrel{\check{\omega}}{=}$ & $\stackrel{\check{\nu}}{\rightleftharpoons}$ \\
\hline 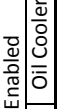 & 2 & $\stackrel{0}{2}$ & $\stackrel{\check{\nu}}{\rightleftharpoons}$ & & $\stackrel{\mathscr{ٌ}}{\rightleftharpoons}$ & 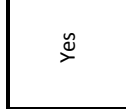 & $\stackrel{y}{9}$ & $\stackrel{\square}{\rightleftharpoons}$ & $\stackrel{\check{ٌ}}{\rightleftharpoons}$ & $\stackrel{\varrho}{\rightleftharpoons}$ & $\stackrel{\check{\varrho}}{=}$ & $\stackrel{\varrho}{\rightleftharpoons}$ \\
\hline $\begin{array}{l}0 \\
\end{array}$ & $\stackrel{2}{2}$ & 2 & $\stackrel{\check{ٌ}}{\rightleftharpoons}$ & & $\stackrel{\check{ٌ}}{\rightleftharpoons}$ & $\stackrel{\varrho}{\rightleftharpoons}$ & $\stackrel{5}{2}=\frac{y}{2}$ & $\stackrel{\square}{\rightleftharpoons}$ & $\stackrel{\check{\Xi}}{=}$ & $\stackrel{\Xi}{=}$ & $\stackrel{\check{\Xi}}{=}$ & $\stackrel{\varrho}{\rightleftharpoons}$ \\
\hline | & $\frac{0}{2}$ & $\stackrel{\mathscr{\nu}}{\check{\nu}}$ & 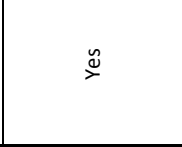 & & 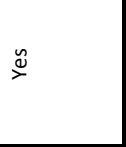 & 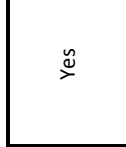 & $\stackrel{\mathscr{\Perp}}{>}$ & $\stackrel{\Delta}{\stackrel{\varpi}{\nu}}$ & $\stackrel{\check{\varpi}}{\rightleftharpoons}$ & $\stackrel{\varrho}{=}$ & $\stackrel{\mathscr{c}}{=}$ & 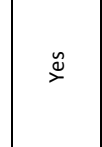 \\
\hline $\begin{array}{l}\dot{1} \\
0 \\
\tilde{0}\end{array}$ & $\frac{\pi}{z} \frac{\pi}{z} \frac{\pi}{z}$ & $\frac{\pi}{z} \frac{\pi}{z} \frac{\pi}{z} \frac{\pi}{z} \frac{\pi}{z}$ & 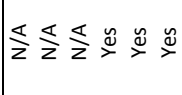 & $\frac{\pi}{z} \frac{\pi}{z} \frac{1}{z}$ & $\frac{\pi}{2} \frac{\pi}{z} \frac{1}{2} \mid \frac{1}{2}$ & 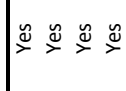 & 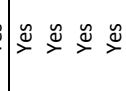 & 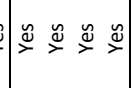 & 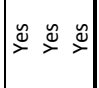 & 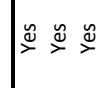 & 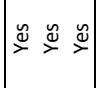 & 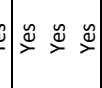 \\
\hline 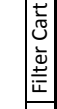 & 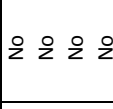 & 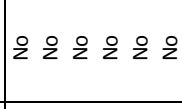 & 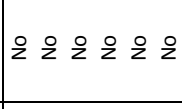 & $\begin{array}{lll}0 & 2 & 2\end{array}$ & 울 $2: 0$ & 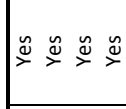 & 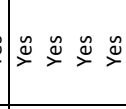 & 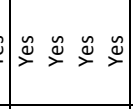 & 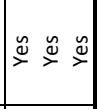 & 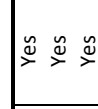 & 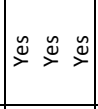 & $\hat{b} \stackrel{\breve{d}}{\rightleftharpoons} \stackrel{\square}{=}$ \\
\hline 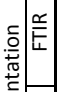 & 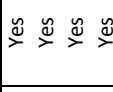 & 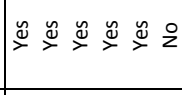 & 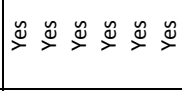 & 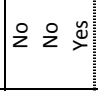 & 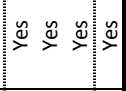 & 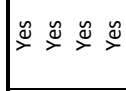 & 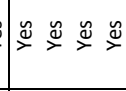 & 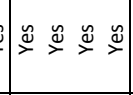 & 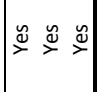 & 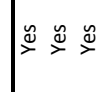 & 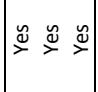 & 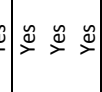 \\
\hline 氙 & 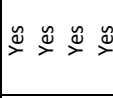 & 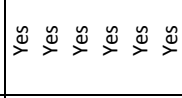 & 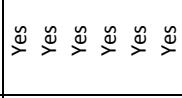 & 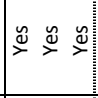 & 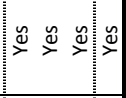 & 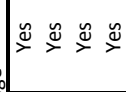 & 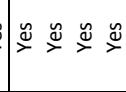 & 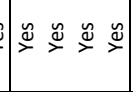 & 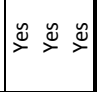 & 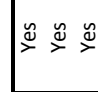 & 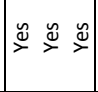 & 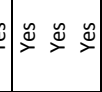 \\
\hline 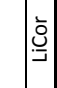 & 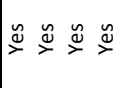 & 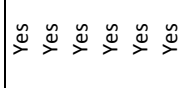 & 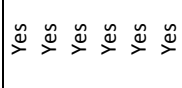 & 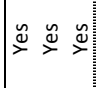 & 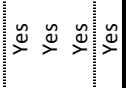 & 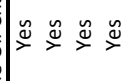 & 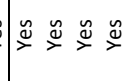 & 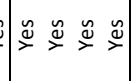 & 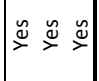 & 竞 & 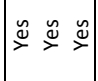 & 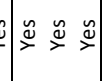 \\
\hline 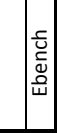 & 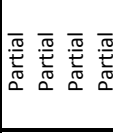 & 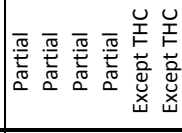 & 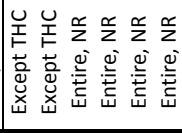 & 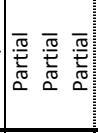 & 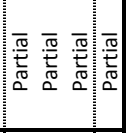 & 总总总总 & 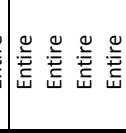 & 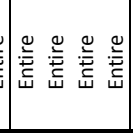 & 善善善 & 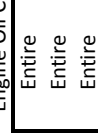 & 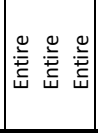 & 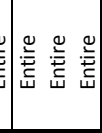 \\
\hline 憘 & 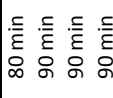 & 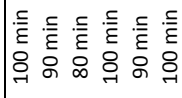 & 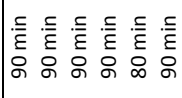 & 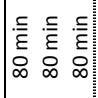 & 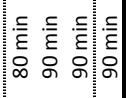 & 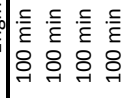 & 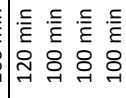 & 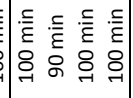 & 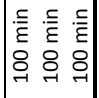 & 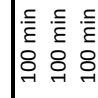 & 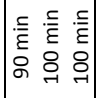 & 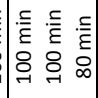 \\
\hline 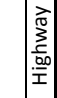 & $\breve{u}$ & 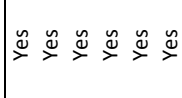 & 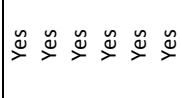 & 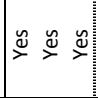 & 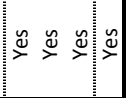 & 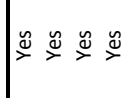 & 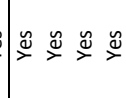 & 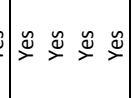 & 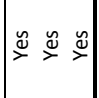 & 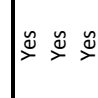 & 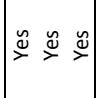 & 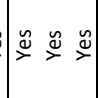 \\
\hline 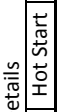 & $\gtreqless 2 ㅇ$ & 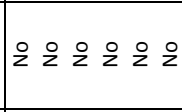 & $\frac{1}{2} z \frac{0}{z} z \frac{0}{z} z$ & 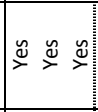 & 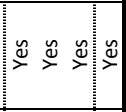 & $\frac{1}{2} \div 2 ㅇ$ & 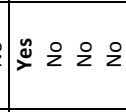 & 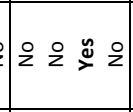 & $200 \stackrel{0}{2}$ & 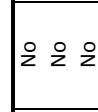 & 욜 & 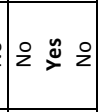 \\
\hline 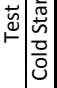 & $\frac{⿱}{z} \frac{⿱}{z} \frac{⿱}{z} \frac{x}{z}$ & $\frac{x}{z} \frac{x}{z} \frac{x}{z} \frac{x}{z} \frac{x}{z} \frac{x}{z}$ & $\frac{\infty}{z} \frac{x}{z} \frac{x}{z} \frac{x}{z} \frac{x}{z} \frac{x}{z}$ & $\frac{⿱}{z} \frac{x}{z} \frac{x}{2}$ & $\frac{\infty}{z} \frac{\infty}{z} \frac{x}{2}\left[\frac{\alpha}{2}\right.$ & 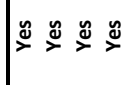 & 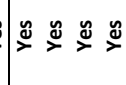 & 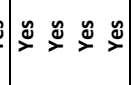 & 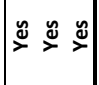 & 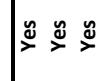 & 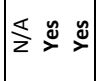 & 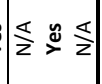 \\
\hline 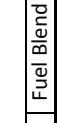 & $\frac{\pi}{z}$ & $\frac{\pi}{z}$ & $\frac{\pi}{z}$ & $\frac{\varangle}{z}$ & 의 원 & $\stackrel{0}{u}$ & 这 & 웜 & 弚 & $\stackrel{0}{u}$ & $\stackrel{9}{1}$ & 总 \\
\hline 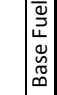 & $\stackrel{\frac{d}{2}}{\vec{g}}$ & $\stackrel{\frac{d}{w}}{\underline{3}}$ & 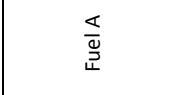 & 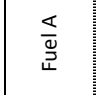 & $\frac{\infty}{\underline{a}}$ & $\frac{\infty}{\underline{m}}$ & $\frac{\infty}{\frac{\omega}{3}}$ & $\frac{\frac{\omega}{\omega}}{\frac{g}{3}}$ & $\frac{\frac{\infty}{\omega}}{\underline{g}}$ & $\frac{\infty}{\frac{m}{3}}$ & 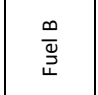 & $\frac{\infty}{\frac{\omega}{u}}$ \\
\hline 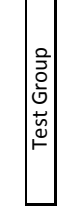 & 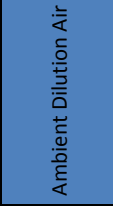 & 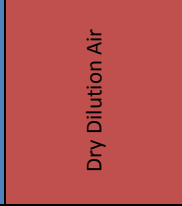 & 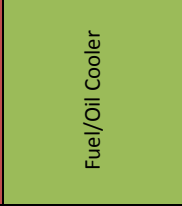 & & 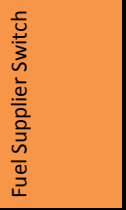 & $\stackrel{\circ}{u}$ & 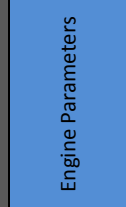 & 웜 & ஜ̊ & 总 & 욤 & 염 \\
\hline 产 & 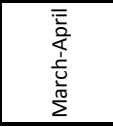 & 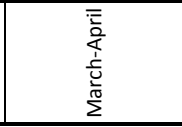 & $\stackrel{\frac{\pi}{0}}{\Sigma}$ & & 焈 & 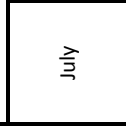 & 言 & 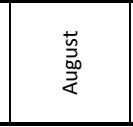 & 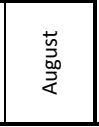 & 喜 & 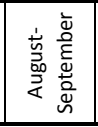 & 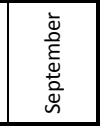 \\
\hline
\end{tabular}


The following provides a breakdown of the columns of the test matrix, including an explanation of the meaning of some of the entries:

Base Fuel: fuel supplier for the base gasoline used (see Section 4.7).

Fuel Blend: ethanol, toluene, or ethanol/toluene fuel blend used (see Section 4.7).

Cold Start: cold start performed or reported?

Hot Start: hot start performed or reported?

Highway: steady-state highway cruise?

Length: overall length of test, not including additional hot start if performed.

Instrumentation: breakdown of which instruments were used/reported.

Ebench: emissions analyzers used; only data with entire emissions bench is reported here.

Partial: $\mathrm{CO}_{2}, \mathrm{O}_{2}, \mathrm{CO}$ analyzers only.

Except THC: all analyzers except for THC.

Modifications: which modifications were enabled.

NR: test result(s) not reported due to issues.

N/A: results not available.

\section{Results Preamble}

To reiterate, the overarching theme of this thesis is to analyze the effects that different parameters have on PM emission repeatability, at otherwise steady and consistent engine operating conditions. The main metrics used therefore, are the total PN concentrations of the different test groups and individual runs, and statistics that describe them from a variation point of view. These include the relative standard deviations and standard errors of test averages as measures of test-to-test variation. Comments on trend statistical significance are supported with calculated p-values. Auxiliary metrics are used here to provide additional context in an attempt to explain findings and analyze their affect on a more fundamental level. Particle size distributions and PM mass/composition, as well as gaseous emissions measurements are used in this light. Consideration is also given to provide commentary on the suitability of ethanol blended fuel as a control technique for PM emissions reductions. This is maintained as the overall goal of this project, with the focus here being on improving the repeatability of the results for future ethanol work. 


\subsection{Systematic Sources of Variability}

Several potential systematic sources of PM emission variability were proposed for investigation in Chapter 3 based on indications from literature. The following presents the findings from the control of those parameters. Data presented in this section is primarily exhaust PN concentrations and particle size distributions provided by the EEPS.

\subsubsection{Dilution Air Humidity}

As first explained in Chapter 3, the dilution air provided by the rotating disk thermodiluter is subject to changes in ambient and test cell humidity. This was therefore identified as being a potential source of measured PM emission variability and was the first systematic parameter investigated. To study this influence, a series of tests were performed with both ambient air and dried dilution air supplied to the diluter and changes in EEPS measurements were noted. The average PN concentration data from these tests is presented in Figure 6.1.

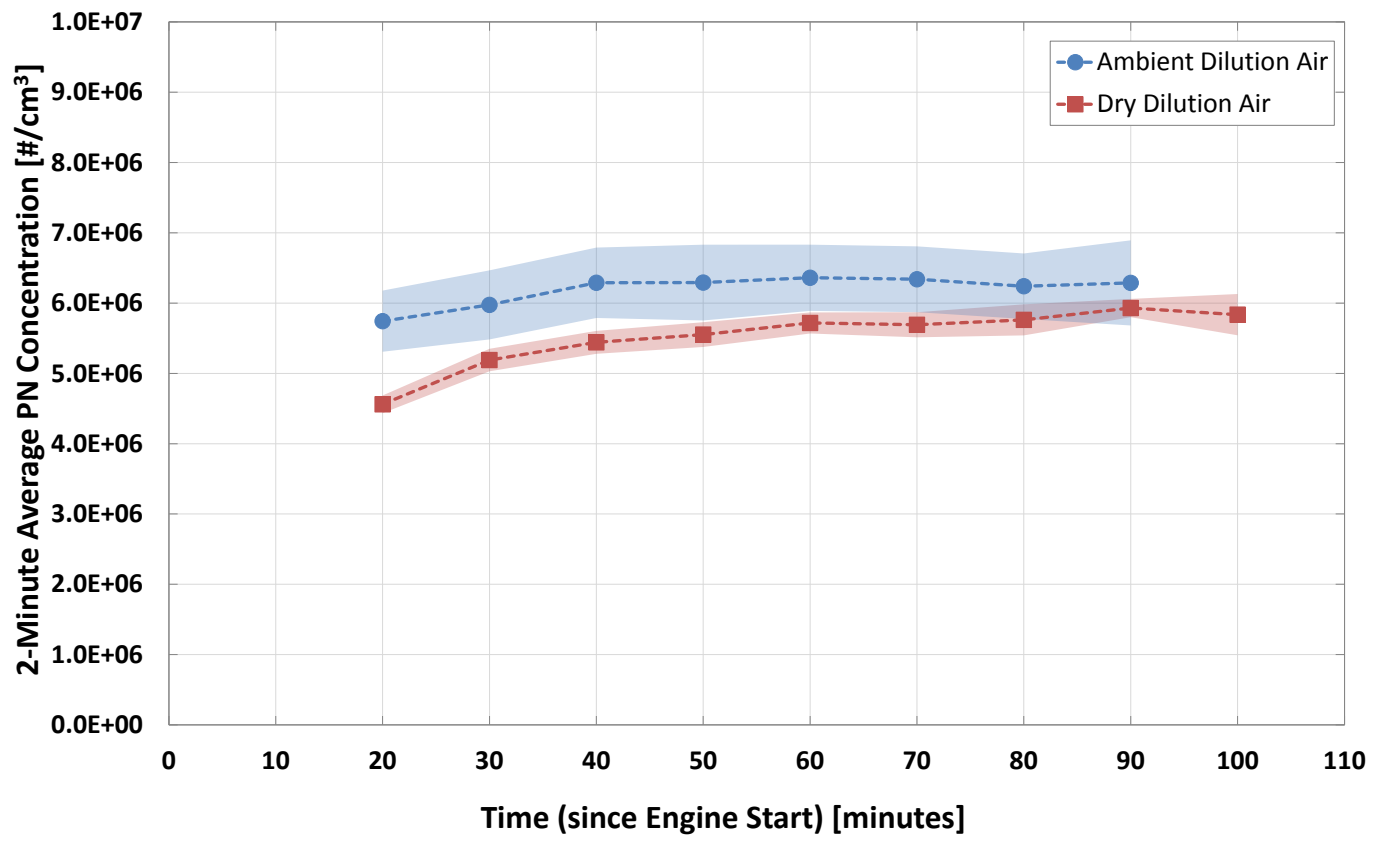

Figure 6.1: Two-minute average PN concentrations for ambient and dry dilution air test groups using Fuel A. Shaded areas indicate standard error.

The ambient dilution air data series represents identical conditions to the data gathered by Mireault [44] presented in Figure 3.1, and therefore serves as a direct comparison. Upon first inspection, the data does not appear to exhibit the same temporal variation (drift upward with run-time) seen in the previous work's data; PN concentrations here were observed to stabilize after 40 minutes of operation, whereas increases continued to occur well after the midpoint of runs of similar length in [44]. This finding in itself is rather peculiar as no systematic or procedural changes were performed for this test group. Changing to the dry dilution air supply resulted in a shift to fewer particle counts; however, the temporal drift upward reappeared with moderate significance $(\mathrm{p}=0.13)$ when considering data from 40-90 minutes. What is also visible is the reduction in test-to-test variation - or in other words, the 
increase in data repeatability - shown by the reduction in the standard error (shaded area) in Figure 6.1. This is quantified in Figure 6.2 with a plot of relative standard deviation (RSD) for the two test groups with time. In this case, an average reduction of $54 \%$ in RSD was observed through a typical test duration. Note that the dry dilution air test group did benefit from a greater sample size (6 vs. 4), though this benefit is inconsequential given the strong statistical significance of the result $(\mathrm{p}<0.01)$.

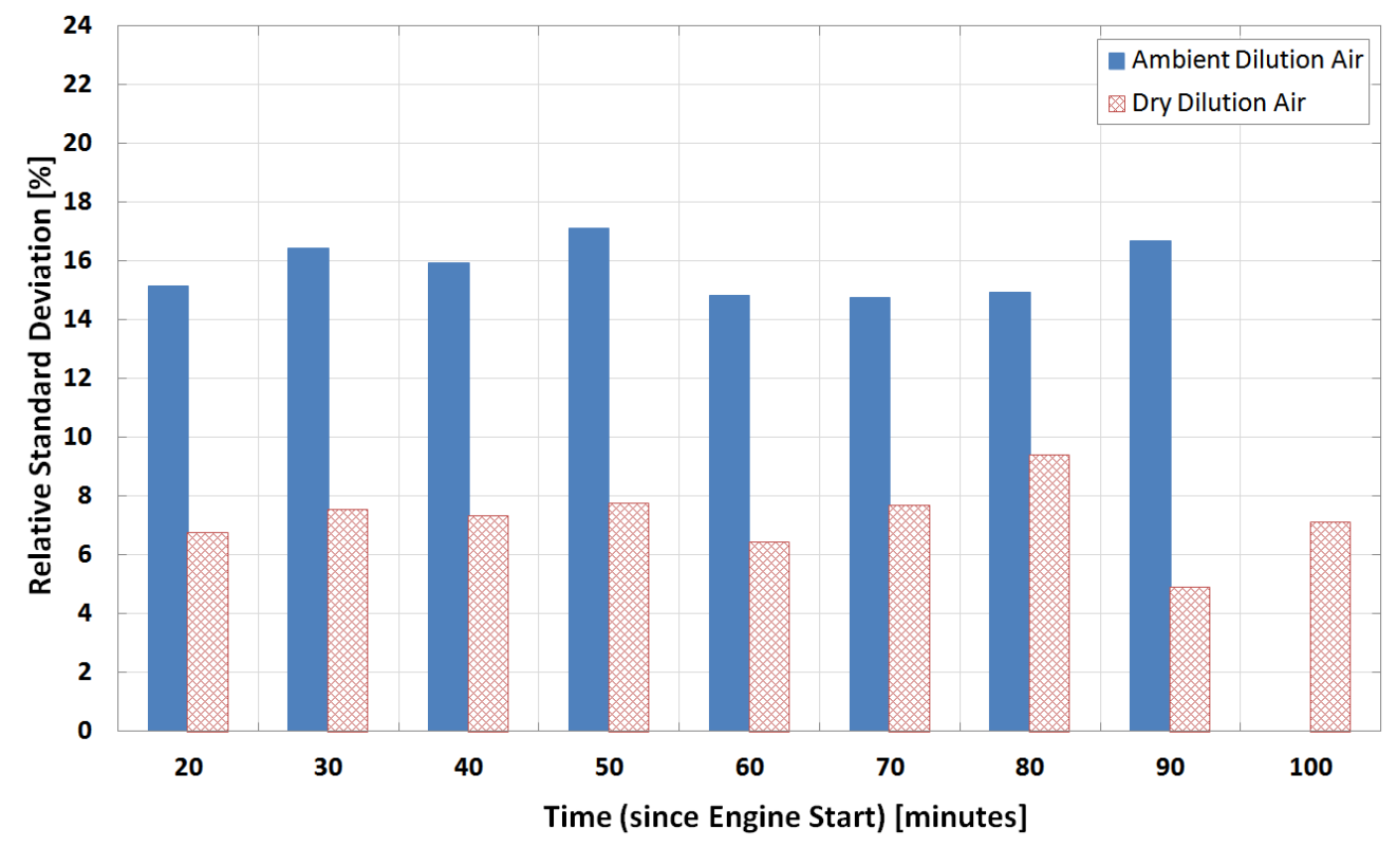

Figure 6.2: Relative standard deviations with test run time for ambient and dry dilution air test groups using Fuel A.

Inspection of the particle size distributions, given in Figure 6.3, show little in terms of differences between using a dry dilution air supply or an ambient air supply. Both distributions display the characteristic shape (accumulation mode dominant) for this engine at this operating condition [44]. Of course, the minor shift to lower total concentrations shown in Figure 6.1 is manifested as lower concentrations at the peaks. In both cases, the modal diameter of the distributions coincided with the $69.8 \mathrm{~nm}$ size bin, while the median diameters (size bin where $50 \%$ of the particles are greater or smaller in size) coincided with the $52.3 \mathrm{~nm}$ bin. Recalling from the literature presented in Chapter 3, increasing the dilution air humidity primarily causes increases in smaller, nucleation mode particles [45, 47, 46]. In this work, this trend was not observed and is confirmed in Figure 6.4, where the normalized particle size distributions are presented. Here, both normalized distributions are very similar in both shape and magnitude leading to the conclusion that all particles were equally affected by the dry dilution air supply within statistical relevance. It should be noted that the above referenced sources investigated influence of dilution conditions on diesel engine particles, which could be the reason for this apparent discrepancy.

The use of dry dilution air, therefore, shows strong improvement in PM emission measurement repeatability (run-to-run variability) from a PN concentration metric perspective. Given the information available, the stability of the humidity of the dilution air supplied by the dry air supply, rather than the 'dryness' of the air itself, is thought to be the reason for this observation. The improvement is quite clear and so the dry dilution air supply was maintained for all further testing in order to offer the best comparison and eliminate as many potential variables as possible. 


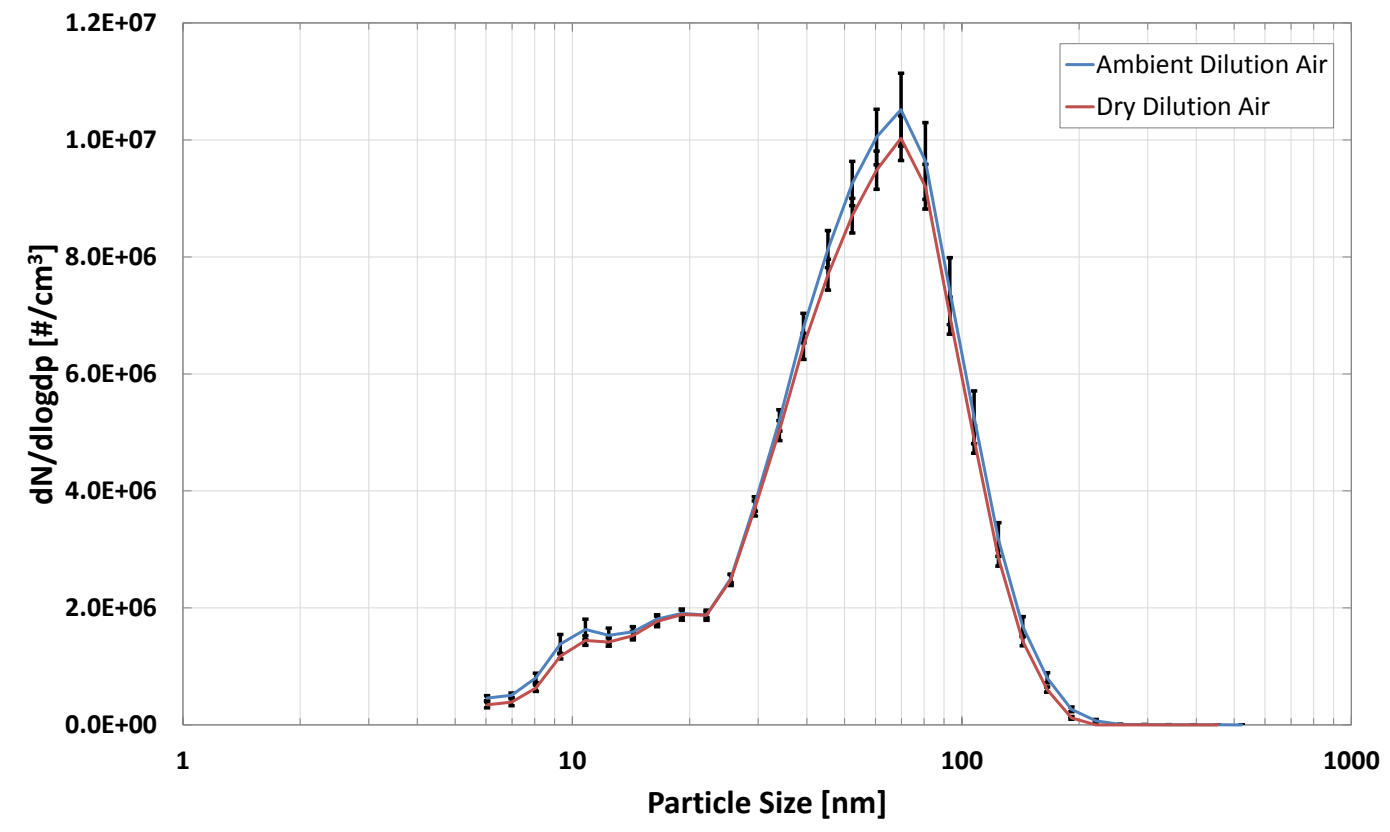

Figure 6.3: Two-minute average particle size distributions for ambient and dry dilution air test groups measured at run-end using Fuel A. Error bars indicate standard error.

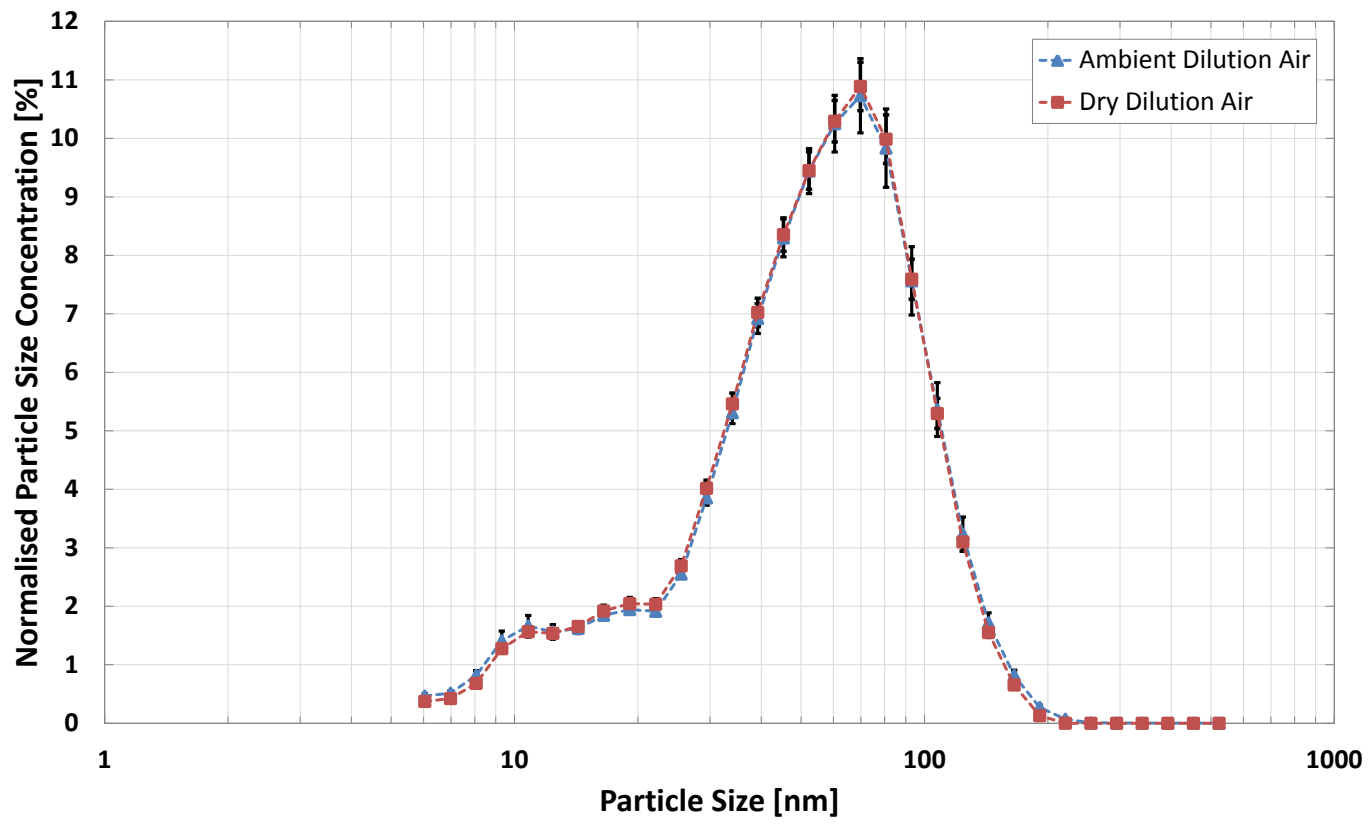

Figure 6.4: Normalized particle size distributions from Figure 6.3. Error bars indicate standard error.

\subsubsection{Fuel Temperature}

Engine temperatures critical to PM formation, including ECT and oil temperature, were shown to be stable at steady-state engine operation in previous work on this research engine [44]. Once engine temperature stabilization is reached, these temperatures remain within the tolerances specified in Table 5.4. In contrast, the fuel temperature does exhibit a temporal drift with run-time on this engine because 
the fuel continually cools the low pressure fuel pump, building up heat in the fuel tank. As previously discussed, the fuel temperature can also play an important role in PM formation [56]. To judge the influence of this temperature change on PM formation from this engine, a test was performed where the fuel supply was switched at the end of a run from the tank with the built up temperature to the second tank with fuel at ambient conditions. Figure 6.5 plots the PN concentration and fuel temperature through this tank switch (represented by the dashed line). As expected the fuel temperatures - measured both in the tank and at the pressure regulator - dropped immediately after the switch. More telling however, is that the measured PN concentration also dropped an appreciable amount, mirroring the result seen by Whelan et al. [56]. Note that a PM emission response was recorded even with a modest change in fuel temperature. Given this evidence, it was thought that the change in fuel temperature over the course of a typical run would, at least in part, account for the increase in measured PN concentrations.

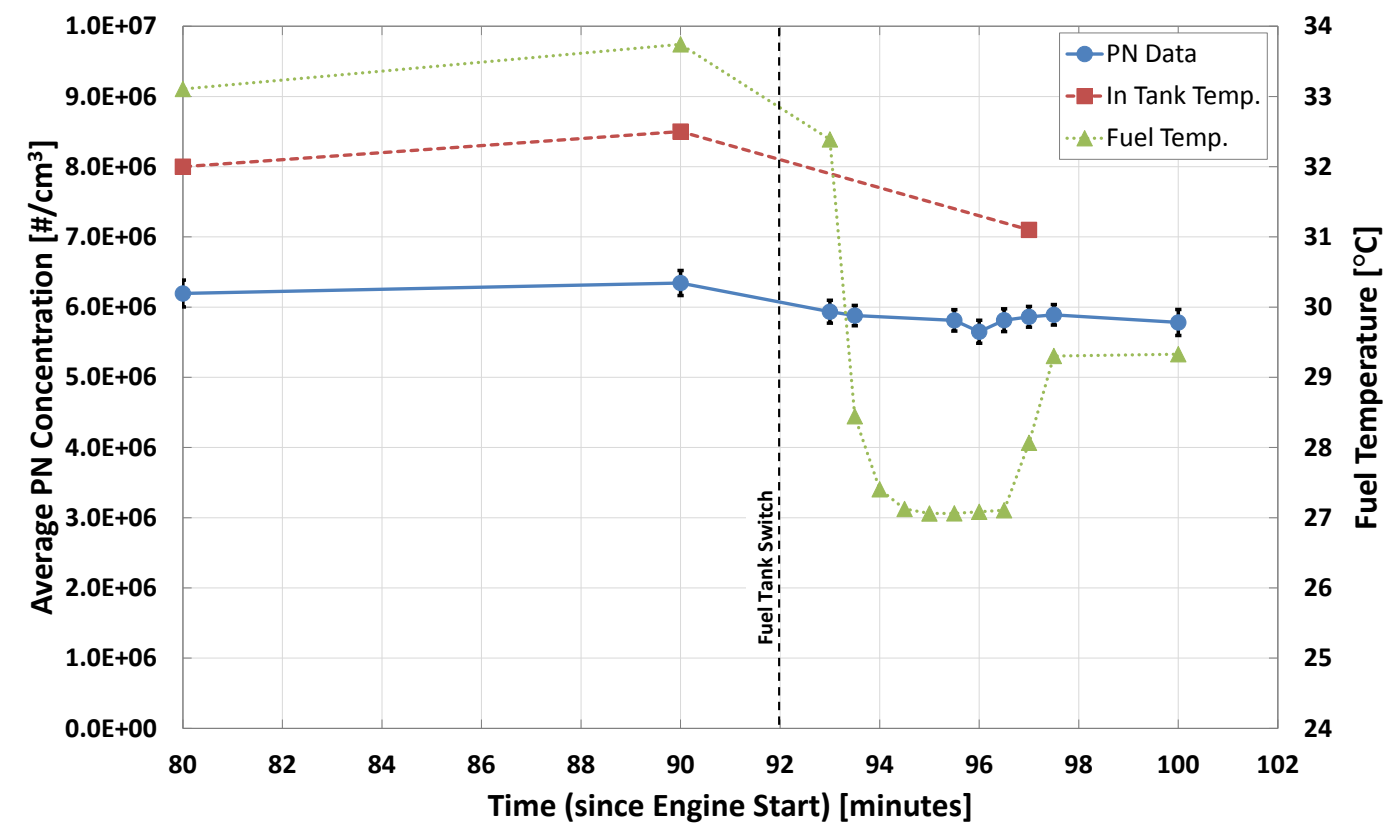

Figure 6.5: PN concentration during a fuel tank switch (drop in fuel temperature) using Fuel A. PN data points before the switch are two minute averages, followed by 30 second averages during the switch. Error bars indicate one standard deviation.

A fuel cooler was consequently designed and installed on this apparatus for investigation as described in Section 4.2.1. In addition to the fuel temperature, the lubrication oil temperature was also hypothesized to potentially play an important role in the temporal variation of PM emissions. Therefore, an oil cooler (see Section 4.2.2 for description) was added in the hopes that it could speed up the stabilization of PM emissions at steady-state operation, as well as to investigate the influence that oil temperature has on PM formation. Figure 6.6 shows the PN concentration results of a series of tests with both the fuel and oil coolers installed on this research engine; the dry dilution air from the previous discussion is used here as a baseline set of values for comparison. Though not plotted here, the fuel and oil temperatures during fuel/oil cooler tests were held nominally at $22{ }^{\circ} \mathrm{C}$ and $99{ }^{\circ} \mathrm{C}$, respectively (see Table 5.4). Visual inspection of the data provides for two major observations: first, the temporal increase in PM emissions appears to be significant $(\mathrm{p}<0.05)$ and stronger for the fuel/oil cooler enabled test group than for the stock set-up; and second, the increased spread of the standard error bounds indicating a 
decrease in PM emission repeatability. This finding directly contradicts the results in Figure 6.5 along with the hypothesis that stabilizing the fuel temperature would have some desirable effect on stabilizing the PM emission rate. It is important to note that the data presented in this figure does not discern the effect of both the fuel and oil coolers individually; this was studied later and is presented in Section 6.1.5. It is possible that whatever positive influence afforded by controlling the fuel temperature was negatively offset - to a greater extent - by the lowered oil temperature, though this is highly speculative given this information. Additionally, given that the oil temperature, while reduced, was still stable during the steady engine operation does not provide for the observed PM emission rate increase. This seemingly unsatisfactory explanation of the observed results, along with the subsequent identification of other potential systematic sources of PM variation, led to leaving both coolers enabled for further work during this investigation.

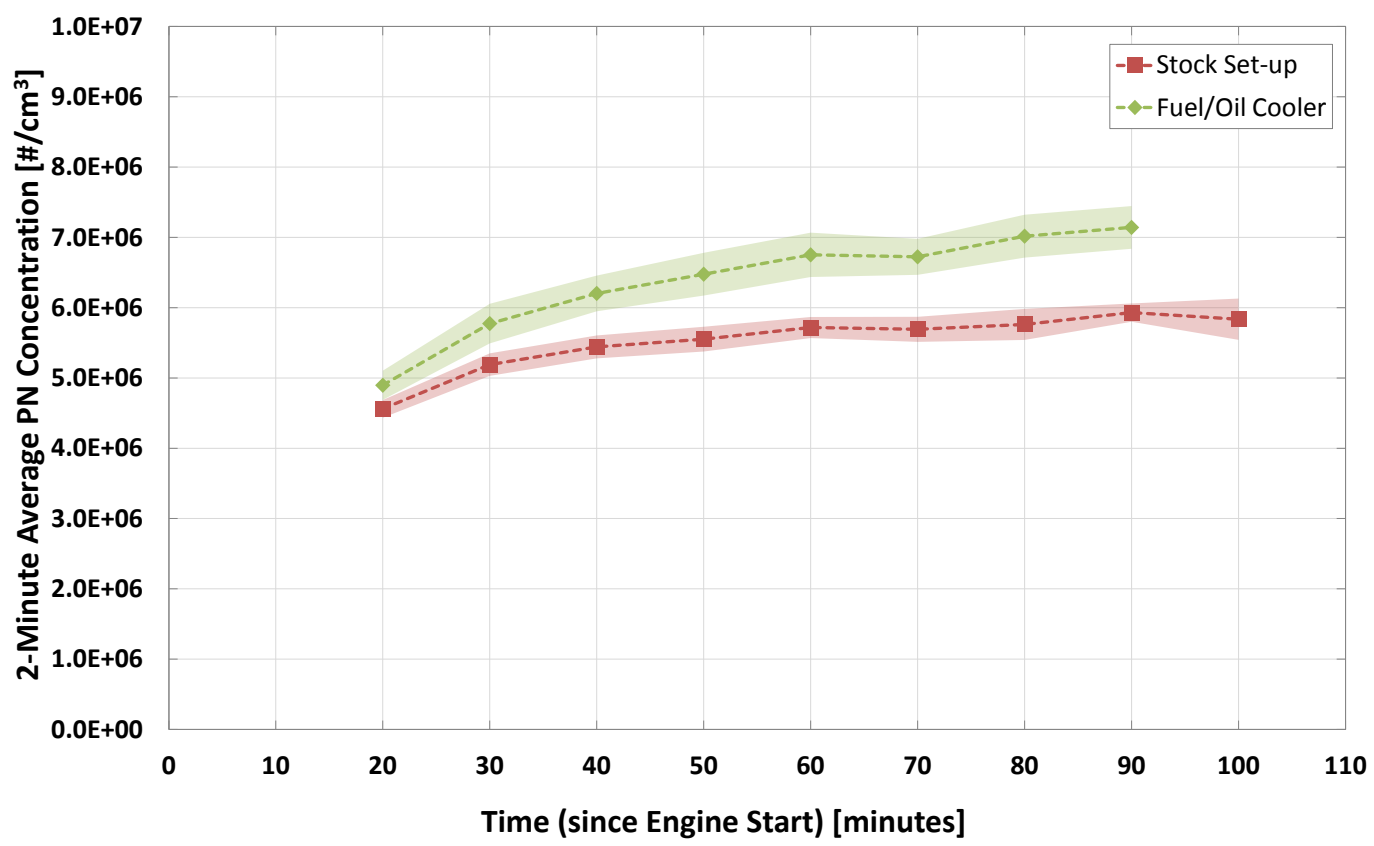

Figure 6.6: Two-minute average PN concentrations for "stock set-up" and both fuel and oil coolers enabled using Fuel A. Shaded areas indicate standard error.

\subsubsection{Engine Deposits and Engine Cleaning}

Engine deposits, including in the induction system and on injector faces, were subsequently identified as potential causes of the regression in PM emission variability, as noted above. As discussed in the literature review, GDI engines inherently suffer from the formation of intake deposits. The working hypothesis here is that while the deposits themselves are not directly contributing to the formation of PM they can be a marker for degrading conditions including valve sealing issues and combustion chamber hot spots, as well as the ingestion of engine lubrication oil into the combustion process. Furthermore, it is not a stretch to consider that flow perturbations caused by these deposits could be altering incylinder flow characteristics, important for fuel-air mixture preparation. It is important to note that the condition of the valves and intake runners prior to the start of this investigation were not equivalent to a fully clean engine. As a rough estimate, approximately $8000 \mathrm{~km}$ of equivalent engine mileage was 
accumulated between the end of Mireault's work [44] and the time this documentation was performed. However, given the progressive nature of carbon build up in engines it is likely that it was "cleaner", at least to some extent, prior to the start of the work detailed in this thesis.

Figure 6.7(a) shows the condition of the intake valves and runners prior to engine cleaning. It is obvious from these pictures that deposits have begun to form on the backs of the intake valves, along with a build up of oil in the runners themselves. The main culprits of deposit formation are oil ingestion into the intake system via the crankcase ventilation system and the back flow of exhaust gases during valve overlap periods. This is of course exacerbated in direct injection engines because fuel is not sprayed over these surfaces, helping to break down and remove the deposits. As this was hypothesized to potentially be the cause of increasing variability of PM emissions, a cleaning procedure was undertaken here.

The engine was cleaned by a combination of mechanical and chemical means as described in Appendix C.1. Figure 6.7(b) shows the intake runners after cleaning using a purpose made engine cleaner. Unfortunately, the efficacy of this procedure is questionable as can be seen from these images in comparison to the ones before cleaning. Figure 6.7(c) shows the runners after full mechanical and chemical cleaning; deposits and adsorbed oil have been effectively removed. Post cleaning emissions measurements were made and are presented in the subsequent section on fuel composition.

\section{Injector Deposit Cleaning}

As a final point of discussion, while the cleaning procedure performed above indirectly cleaned the injectors, no physical cleaning of the injectors was performed here. Recalling from an earlier discussion, injector deposits have been shown in previous work to directly impact PM emissions from GDI vehicles, with deposited injectors exhibiting a temporal variation similar to what has been observed here. Issues arose in trying to obtain the necessary parts to perform full injector rebuilds, so instead of cleaning the injectors only deposit documentation was performed. In-cylinder pictures were taken with an illuminated bore-scope using the spark plug port as the access point during the back half of this investigation; see Figure 6.8.

From the images, it is apparent that this injector face was not continually building up soot through the two months of testing that these pictures cover. Both Berkemeier et al. [49] and Berndorfer et al. [48] saw a PM emissions drift when going from fully cleaned injectors to coked ones. The lack of continual build up here indicates that the injectors had likely reached their deposit "equilibrium" and so run-to-run variations or the temporal PM emission increase were likely not from this source. This of course is not to say that cleaned injectors would not cause a similar PM emission behaviour seen in $[48,49]$ on this engine. As further evidence, the injector valve pintle remains unobstructed in all three photos, as does the flow path directly in front of the injector, so a deterioration of flow conditions is also ruled out. Unfortunately this documentation does not permit the discussion of injector plugging (internal deposits) as a source of variability, but the injector face deposits are likely a non-factor in the observed variability in this work. For the sake of completion it would still be beneficial to investigate the matter of injector plugging further on this research engine, especially given the importance of fuel composition on plugging effects $[50,51,52,53]$. 


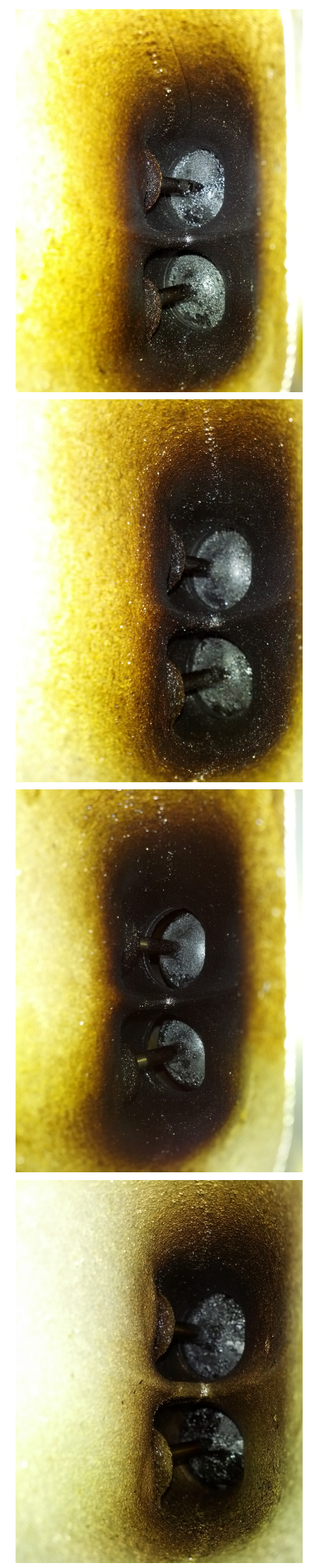

ङ
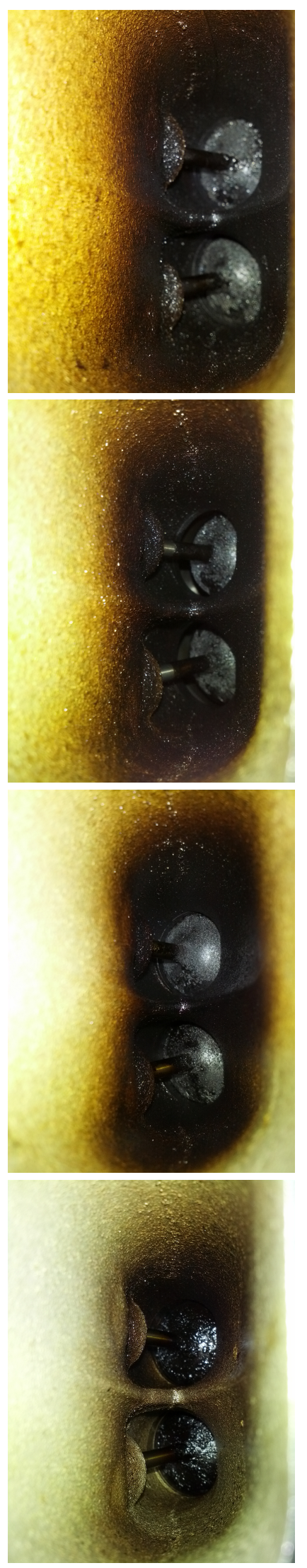

$\hat{e}$

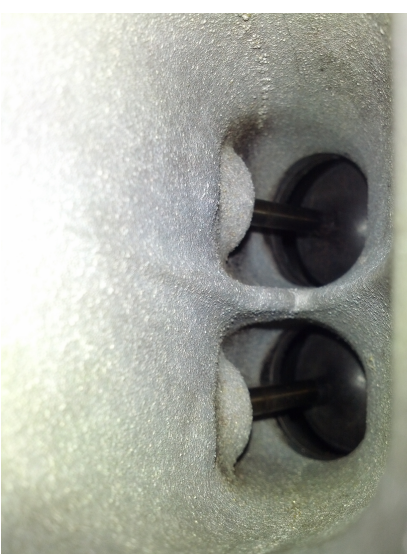

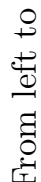
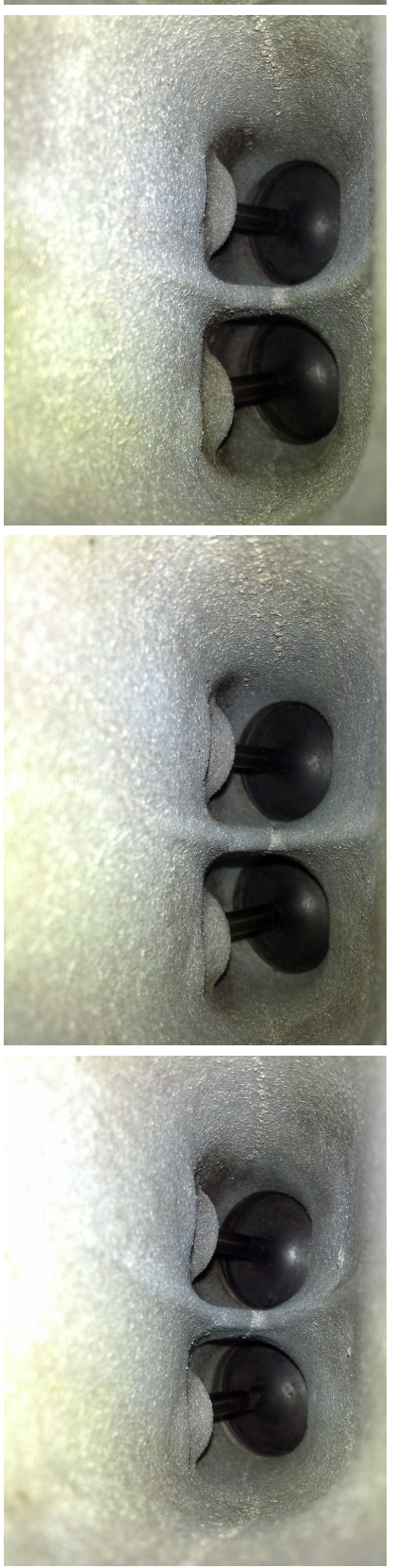

e 


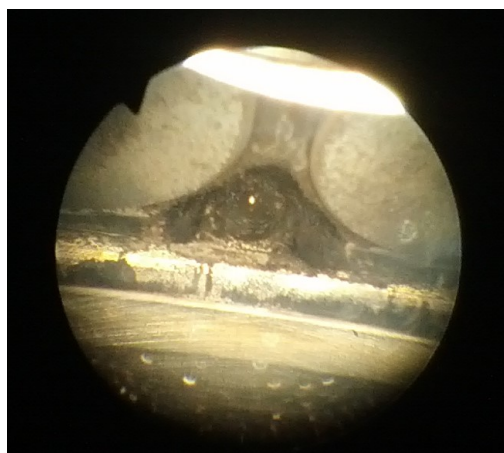

(a)

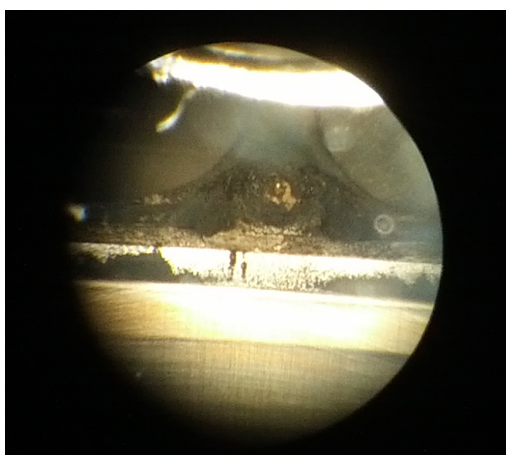

(b)

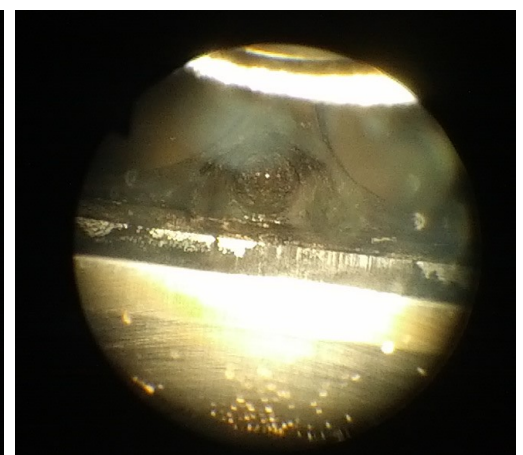

(c)

Figure 6.8: Cylinder 2 injector face images taken (a) after Engine Parameters, (b) after E30, and (c) after T10E10 test groups. Note: camera and lighting settings were not consistent for all pictures.

\subsubsection{Engine PCV Filter}

To prevent further oil ingestion and deposit formation after cleaning, a PCV filter was fitted to the crankcase ventilation system, as previously described in Section 4.2.3. This was done to reduce the formation of deposits and also mitigate the intake of oil into the combustion process - a known source of PM emissions in internal combustion engines. Use of the PCV filter largely mitigated the subsequent formation of induction system deposits as shown by the images in Figure 6.9. These images were taken over the same period as the injectors shown in the previous section, and cover a wide range of fuel blends. Compared to the deposits shown in Figure 6.7 (rightmost column for cylinder 4), no major deposits have formed and the valve condition is largely the same as it was immediately following the manual engine cleaning. Inspection of Figure 6.10 indicates the efficacy of the PCV filter at removing oil vapours from returning to the intake port. The oil which has begun to saturate in the used filter would have otherwise ended up returning to the intake manifold, causing the formation of deposits or involving itself in the combustion process.

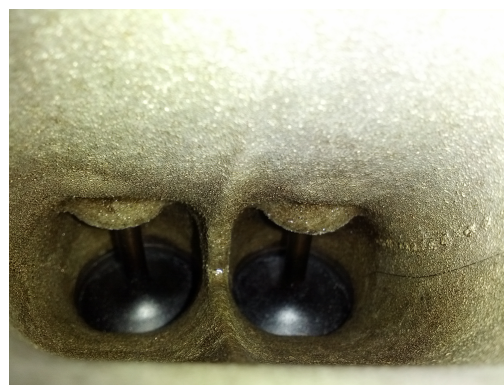

(a)

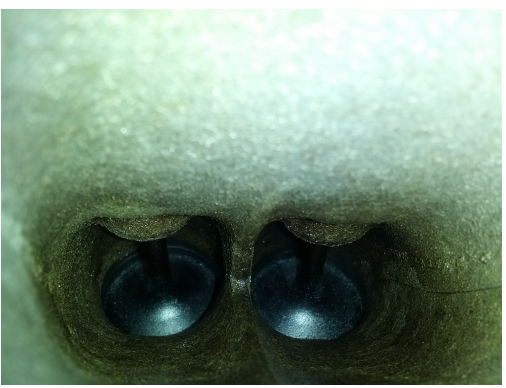

(b)

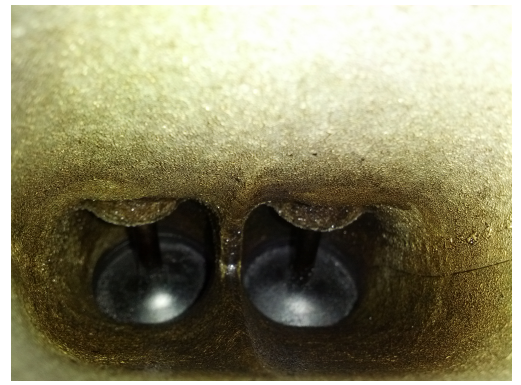

(c)

Figure 6.9: Cylinder 4 intake runners and valves taken (a) after Engine Parameters, (b) after E30, and (c) after T10E10 test groups. Note: camera and lighting settings were not consistent for all pictures.

Even when considering the information these images present, it can still be said that induction system deposits are probably not a leading source of the PM emission variability seen on this engine; these pictures evidence the mitigation of deposit formation, yet the engine still experienced large variations in PM emissions, as will be explained in Section 6.2. While eliminating this source of oil from the combustion process probably eliminated this source of PM emissions, it is likely to not have influenced the emissions at the same level of magnitude of the variations. 


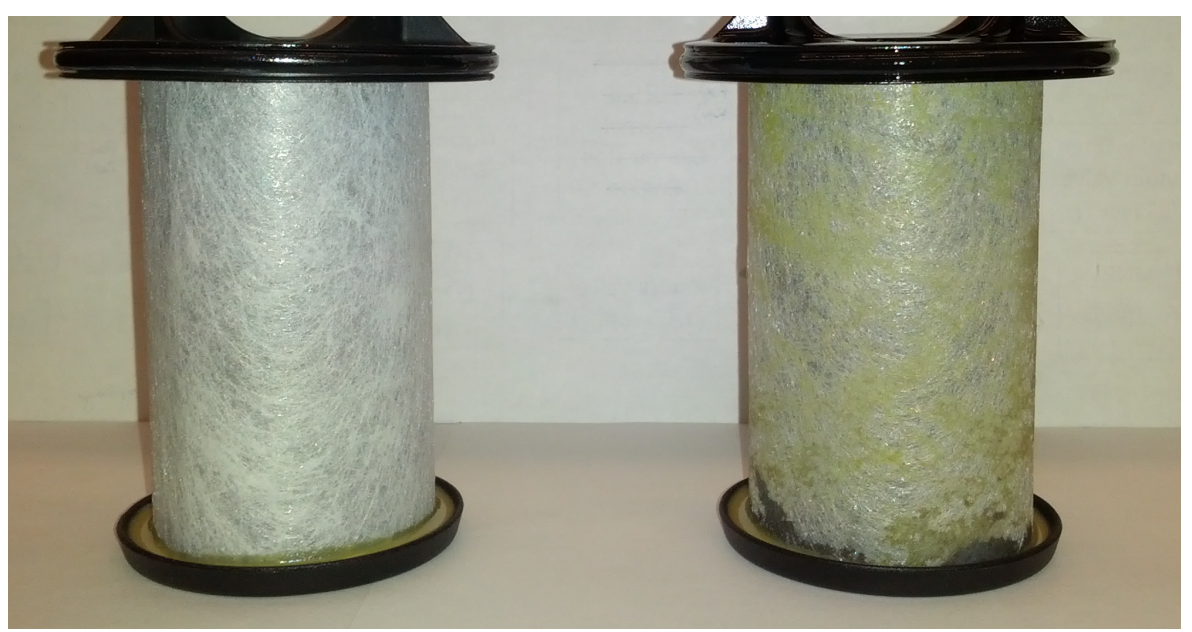

Figure 6.10: PCV filter before use (left) and after E10 test group (right). Absorbed oil shown in right image by spots yellowish in colour.

\subsubsection{Engine Parameters (Re-visited)}

With the intake deposits removed and the PCV filter installed, the influence of fuel and oil temperatures were revisited, along with the effect of having a short hot-soak period between two steady-state runs. The temperature parameters were investigated by disabling their respective coolers and performing a run; note that typical temperatures for both situations are listed in Table 5.4. The data presented in Figure 6.11 shows the results from these four individual tests. The fuel for this test is different then tests performed previously (E0 vs. Fuel A) so a direct comparison is not useful. Instead, comparisons should be made to the E0 data series presented in the fuel composition discussion in Section 6.2.

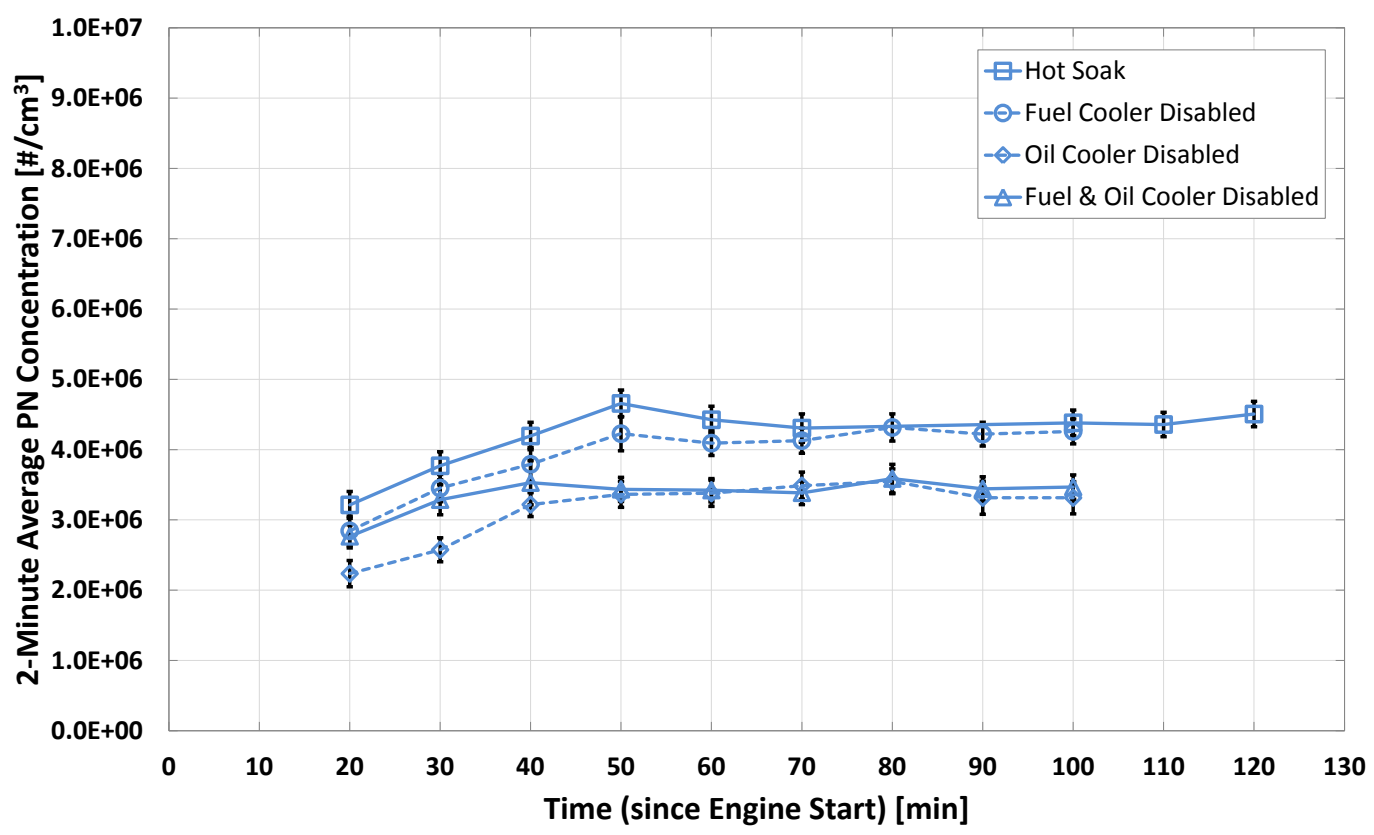

Figure 6.11: Two-minute average PN concentration with test run-time for engine parameter tests with E0 fuel. Error bars indicate one standard deviation. 
The first parameter investigated was the influence of a short, hot soak between two steady-state runs. The procedure for this one test deviates from the one presented in Chapter 5 . Here the engine was started from a cold soak condition and run at highway cruise for two intervals (70 and 40 minutes), with a hot-start procedure performed between them at the 80 minute mark. Comparing the data before and after the hot start shows no appreciable change in PM emission level. The influence of the hot soak can be therefore said to only affect the amount of time to reach steady temperatures, with this occurring much sooner with a hot engine. The following three tests involved disabling the two coolers individually and together to discern their effects; the hot-soak run is used here as a baseline comparison of emissions.

Having just the fuel cooler disabled resulted in no appreciable difference considering the standard deviations between the two runs. This was likely due to the small difference in temperature that the fuel cooler provides (approximately $12{ }^{\circ} \mathrm{C}$ ), albeit with improved stability (i.e. no drift). In contrast to this, when the oil cooler was disabled a different PM emission behaviour was seen. Firstly, once PM emissions stabilization happened it is obvious from this figure that PM emissions were reduced when the oil cooler was disabled. In this case, average reductions of $25 \%$ were observed when compared to the hot-soak run. Recalling that disabling the oil cooler has the effect of raising the steady-state oil temperature approximately $6{ }^{\circ} \mathrm{C}$ (or $6 \%$ ), it is clear that a hotter oil temperature provides positive reductions in PM emissions. Consulting the workshop manual [89] for this research engine provides a logical explanation for this. In this engine, the piston pins are held in place with clips (floating) instead of being pressed in (semi-floating). This difference has implications on the lubrication system used to deliver oil to the bearings at this location. In cases of clipped pins, a drilled hole from the crank end of the connecting rod to the piston pin is typically used to provide this oil to the pin and bearing, whereas a pressed system usually relies on splashing oil making its way to this location. The drilled hole is end-to-end on the connecting rod, meaning that the oil will have direct access to back of the piston crown, so it holds that if the bulk oil temperature is higher, so too will be the piston crown $^{1}$. Assuming that fuel impingement is otherwise constant, the case with the hotter piston crown will evaporate impinged fuel more readily and limit the occurrence of pool fires leading to PM production. To explain why a small difference in temperature appears to have such a pronounced effect on PM emissions requires the consideration of the typical distillation curve of gasoline fuel. Since gasoline is a mixture of many compounds its distillation curve is typically neither flat nor monotonically increasing, especially at the extrema. Therefore it is possible that at this temperature range $\left(99-105{ }^{\circ} \mathrm{C}\right)$ the distillation curve behaves in such a way that a disproportionate amount of fuel is vaporized, hence the exaggerated effect. Finally, disabling both the fuel and oil coolers showed once again that the fuel temperature control has little to no effect on the measured PN concentrations at fully warmed, steady-state conditions. There does appear to be a difference in emission behaviour early in the run, though no explanation can be afforded for this given the available information.

A final remark; even though the fuel and oil coolers provide no benefit from the perspective of reducing PM emissions, they are justifiably used in the remainder of this investigation for the sake of eliminating potential sources of variation and providing finer control over the research engine. Therefore, for the remainder of the tests performed in this investigation, both coolers were left enabled.

${ }^{1}$ The piston crown is the surface directly in contact with the combustion chamber. 


\subsubsection{Engine Oil Age}

The final systematic parameter investigated here was the influence of the engine lubricating oil age. As previously discussed in the literature review, the age of the lubricating oil can influence the emission of PM from engines. Two oil changes were performed during this investigation (see Figure 6.1); the first came at the required service interval for this engine (and after cleaning the intake deposits), while the second was performed after the ethanol fuel blend tests (E10 and E30). The second oil change was not initially planned but provided for an additional collection period and proves useful for comparison here. The results of these two oil changes are presented in Figure 6.12, with the corresponding runs overlaid for clarity. Unfortunately, due to issues with the installation of the aforementioned PCV filter causing the engine to run rich, the first run after the first oil change is omitted. Likewise, the second oil change data series does not include a fourth run due to time constraints. The data presented in this figure represents the normalized PN emissions as a percentage of maximum emission in each data series (663 equivalent $\mathrm{km}$ of data) to remove the issue of magnitude differences.

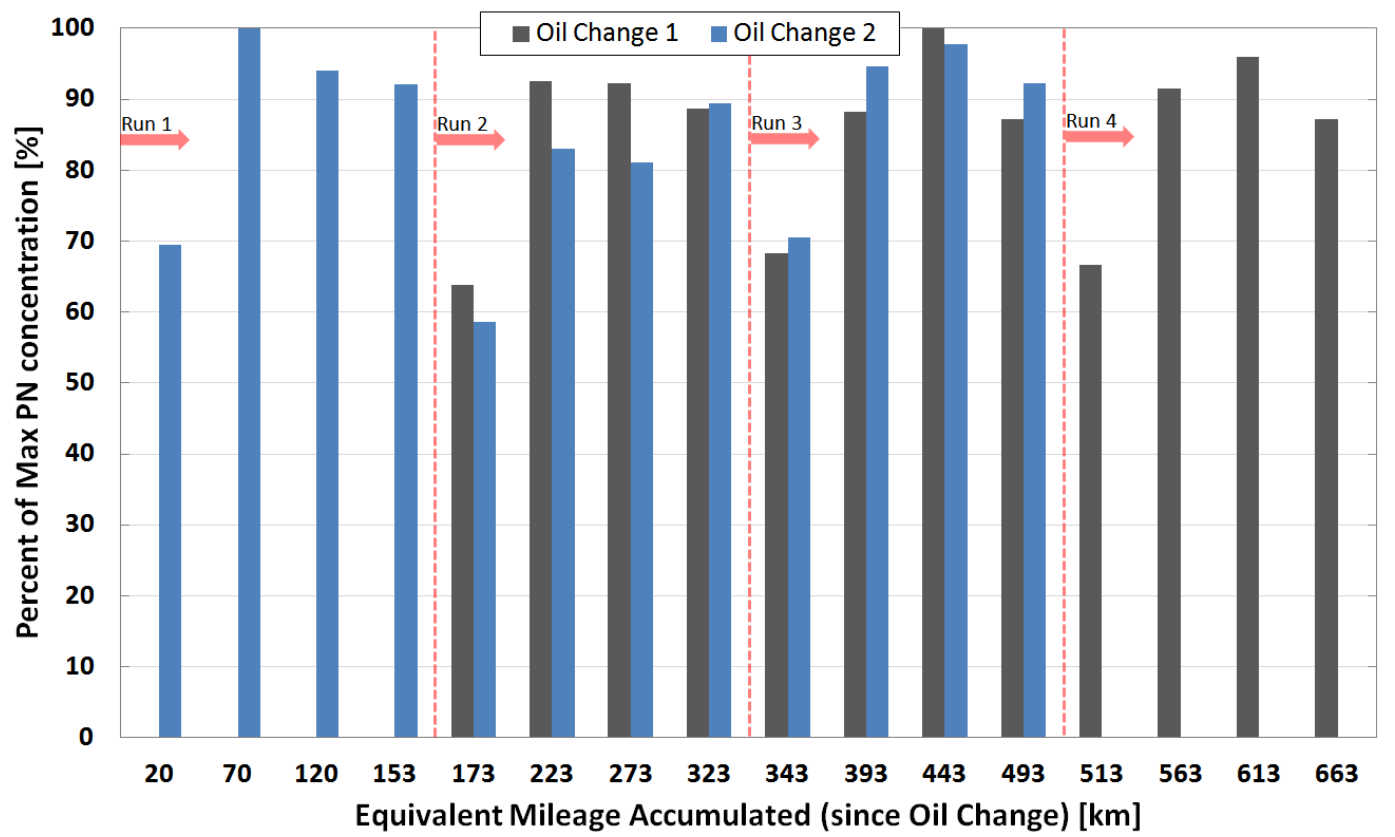

Figure 6.12: Two-minute average PN concentrations with mileage accumulation for the two oil changes performed.

The low emission levels associated with the beginnings of each run (i.e. the first $20 \mathrm{~km}$ of a run) are typical and merely a result of engine warm-up and PM emission stabilization; with respect to the discussion of oil age, this is not important. More relevant is the overall behaviour as the engine oil ages. The work of Christianson et al. [57] shows a period of oil break-in, where the PM emissions decreased by $25-50 \%$ depending on the vehicle to a point of stabilization after $800 \mathrm{~km}$. This is not seen in Figure 6.12 for either data series, both in terms of magnitude and trend. There are, however, some important distinctions to consider. First, the simplified drive cycle performed here is arguably much easier on an engine than the FTP-75 cycles performed in the aforementioned study. Therefore, it is possible that they observed a more pronounced response because of this. Additionally, their work was carried out over a much longer period with larger mileage accumulation, so it also possible that this played into 
their findings. Regardless, if a discernible effect were to be present here, some decay would likely have occurred in the first 500-600 km, but this is not the case. Thus, on the basis of the presented results, the oil age does not appear to affect the PM emissions from this engine.

\subsection{Fuel Composition}

The second major component of this investigation was the inquiry into fuel effects on PM formation and PM emission repeatability. At the onset of this work, only ethanol blended fuels were to be studied. However, given the soon to be described results, further testing was performed by varying other aspects of the test fuel composition, namely the aromatic fraction. Data presented in this and the following sections are grouped by the test fuel used; these test fuels form the test groups referred to in the test matrix in Figure 6.1.

\subsubsection{PN Concentration}

Figure 6.13 contains the test group averaged PN concentration measurements with run time. As before, data points indicate a two minute average at the respective time during the run and the shaded regions indicate the standard error of these test group averaged data sets. Additional statistical information on these data sets is presented in Figure 6.14 where the test group averaged RSD values with run time are provided.

Inspecting these two plots of data shows some intriguing observations. Chronologically speaking, the E0 data shown in Figure 6.13 was taken after performing the engine cleaning and fitting the PCV filter described in the previous section. Looking solely at this data set, it appears that both the problem of run-to-run variability and the typical drifting behaviour had been solved. Indeed, inspecting the data more closely shows that no significant $(\mathrm{p} \approx 0.35)$ drifting with run time (over 40-100 minutes) had occurred during these tests. Additionally, the standard error bounds are substantially reduced over previous data sets presented in Section 6.1. Quantification of this data spread in Figure 6.14 shows approximately a $60 \%$ reduction in calculated RSD when compared to data in Section 6.1. From this information alone, it was believed at the time that both the PCV filter and the engine cleaning had effectively solved the issues at hand. However, investigating the influence of ethanol on PM emissions gave an unexpected response.

Under the same conditions (steady-state, highway cruise) with E10 caused an average increase in measured PN concentrations of approximately $67 \%$, with E30 causing a further increase of approximately $33 \%$ in emission levels. More relevant to this discussion, and objectively more troubling is the observed increase in standard error bounds in Figure 6.13 as an indicator for reduced data repeatability. Indeed, RSD values were calculated to be on average a factor of 4.5 greater for the E10 test group over the initial E0 data set, with the E30 test group showing a factor of 8.3 difference on average. In terms of drifting behaviour, visually comparing the three data sets shows stronger temporal variations with increasing ethanol content. Based on the PN concentrations alone, no explanation could be offered for the observed increases, so a return to E0 fuel was performed to see if emission levels would return to the initial E0 levels.

As can be seen in Figure 6.13, the PM emission levels did not return to the values initially seen in the E0 data set. It is important to remember that the fuel used in both the E0 and E0 Return test groups was from the same commercial gasoline supplier and only obtained a few weeks apart, so no purposeful 


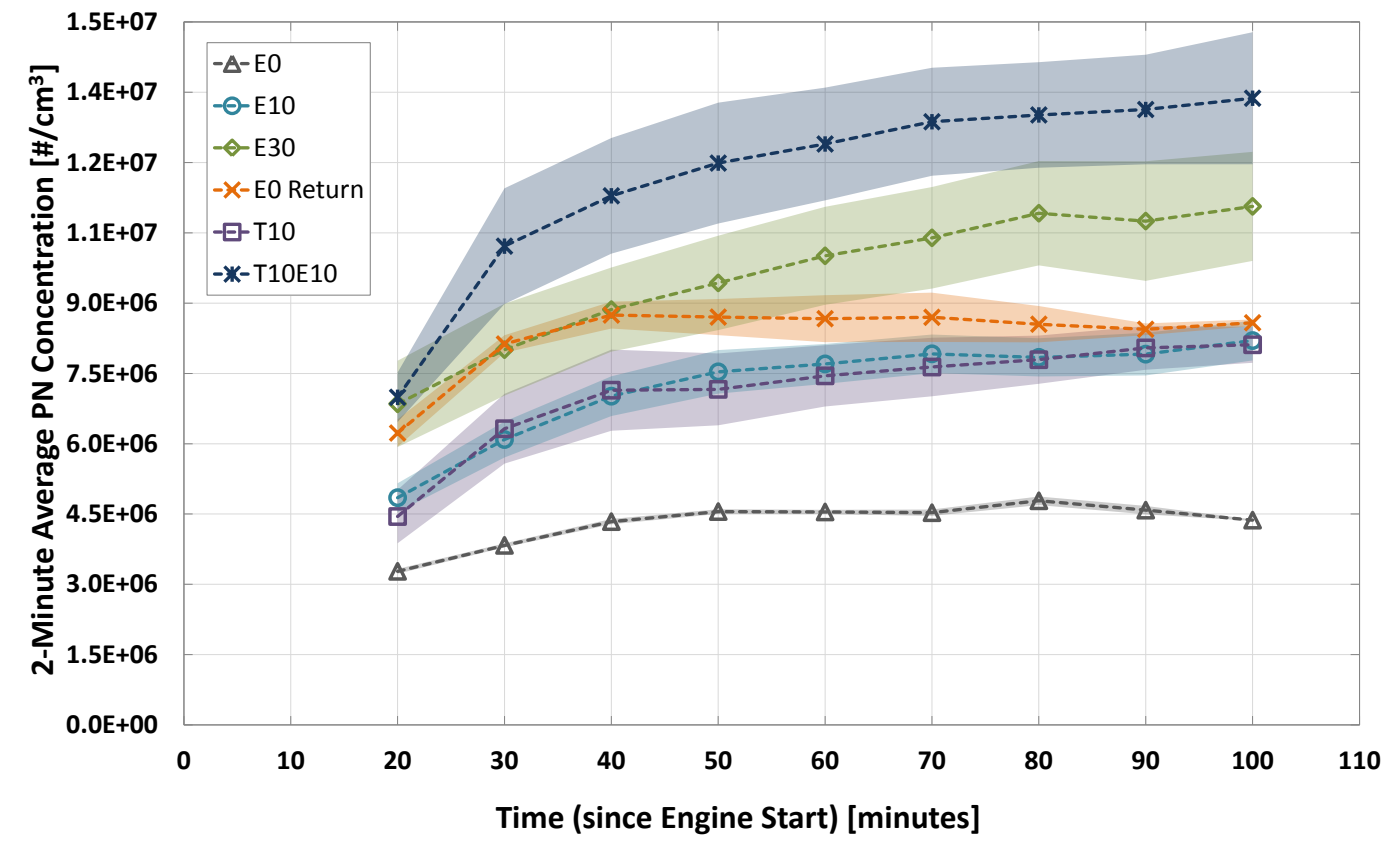

Figure 6.13: Two-minute average PN concentrations for each test group of the different fuel blends. Shaded areas indicate standard error.

change in fuel composition was done. Comparing these two results more closely shows total particle counts of the E0 Return series to be on average almost two times greater than the initial E0 data set. RSD values in Figure 6.14 for this E0 repeat increased an average of 1.6 times over the initial E0 datum, showing that repeatability also did not return to initial values. Compared to the two oxygenate fuels (E10 and E30), the E0 return test group appears to split the difference in PM emissions, emitting more than the E10 test group and less than the E30 test group on average; however, it should be noted that these series do show some overlap, so the confidence of this assertion varies depending on the test time. What is clear however, is the reduction of the RSD of the average PN concentration measurements when returning to E0 from E10 or E30. It is clear from this data that the fuel composition plays an important role in the variability of the PM emissions from this engine. Further, given the large difference between the E0 and E0 Return data it was hypothesized that perhaps other fuel properties are also relevant. Anecdotally, the aroma of the gasoline was noted to differ quite substantially so it was hypothesized that perhaps the aromatic fraction the fuel had changed. To test this hypothesis, two toluene based fuel blends - T10 and T10E10 - were investigated to see how their emissions would fair. Inspecting the two figures here shows that T10 emitted in a very similar fashion to the E10 test fuel, while the T10E10 test fuel emitted the greatest amount of PM of all the test fuels. In terms of variability, both toluene based fuels suffered from a decrease in repeatability, with both fuels giving increased RSD values when compared to the other fuels except E30. The expected result here is that the T10 and T10E10 blends should emit at elevated rates compared to the other fuels, given that they contain a higher aromatic fraction, known for its propensity to form soot. However, the fact that the T10 blend seems to have emitted at a lower rate than the E0 return data is surprising, and not readily explainable with just this data. The T10E10 is intriguing for other reasons. The fact that the PN concentrations climb above the other test fuels, including both the E10 and T10 fuels which the T10E10 is based on, seems to 
predicate a combined effect of ethanol and toluene. A similar conclusion was provided by Capatano et al. [90], where ethanol addition was found to create favourable conditions for the particle formation from the other compounds with higher sooting propensity. He et al. [91] express a similar view in that the slow vaporization of ethanol (high latent heat of vaporization) slows the vaporization of the other components, increasing the possibility of fuel rich cores leading to PM production.

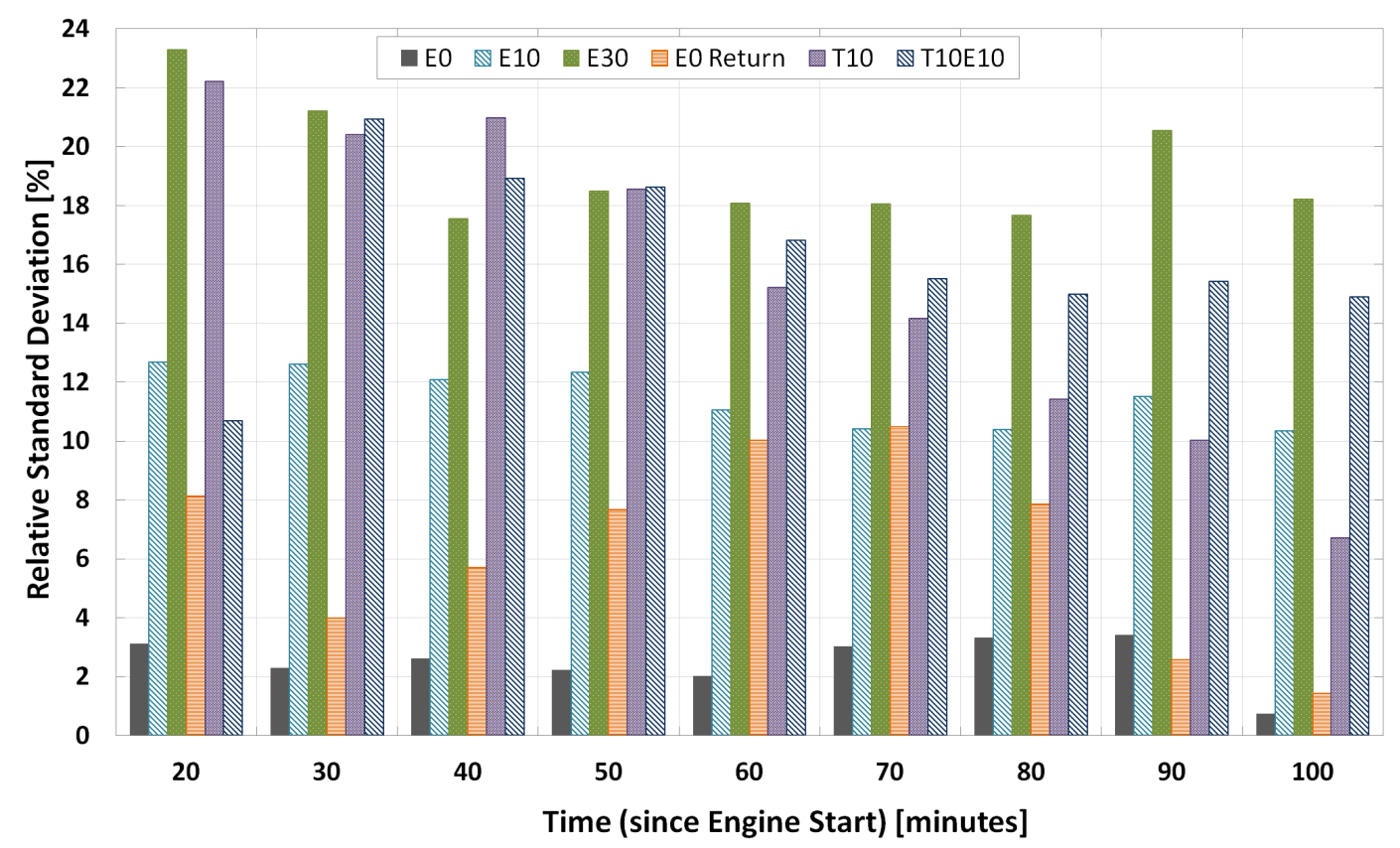

Figure 6.14: Relative standard deviations with test run-time for each fuel blend test group.

\section{Engine Behaviour with Changing Fuel Composition}

The independent conclusions of Capatano et al. [90] and He et al. [91] provided for a couple of engine behaviour hypotheses to explain the observed trends. Specifically, the idea that perhaps the engine or PCM was adapting to the fuel in some way was explored. With regards to the temporal variation of PM emissions, the short-term fuel trim (STFT) engine parameter for the PCM (available from the OBDII data stream) exhibited a similar drift upward with run-time. Additionally, the magnitudes of the fuel trimming appeared to correlate well with increasing ethanol content; i.e. increased trimming levels for increased ethanol. A detailed discussion on the nuances of fuel trimming as it pertains to this discussion is provided in Appendix C.2. To summarize, while the STFT did exhibit a similar temporal variation, when these behaviours were normalised to maximum values the PN emissions drift did not correspond to the STFT drift based on the fuel blend. As an example, the E0 Return test group reached 90\% of maximum PN emissions sooner than all the other test groups, yet on a STFT basis it was the third slowest to $90 \%$ of maximum STFT. This discrepancy leads to the conclusion that the STFT is not a good indication for the observed temporal variation even though they exhibit similar behaviours. As a final note, the long-term fuel trim (LTFT), which provides trimming adjustments at a higher, more permanent level, appears to not have been functioning. Issues with the supplied engine wiring harness were identified as the leading culprit, though it is also possible that it is disabled on this PCM entirely. A properly functioning LTFT could provide the necessary learning for the engine to adapt to the different 
fuel blends after a few runs, and would limit the amount of trimming change required over the course of a run.

A second theory, on the basis of changing combustion stability, was explored to explain the increased variability of $\mathrm{PN}$ concentrations with increased ethanol content. Combustion variability is commonly measured by the cyclic variability of in-cylinder pressure data, expressed as the coefficient of variation of indicated mean effective pressure [8]. Since pressure data was not available on this apparatus, the exhaust temperature was chosen as an analogous metric on the basis that increased CoV of IMEPs follow slow burn situations [8], resulting in higher exhaust temperature. Therefore, the hypothesis follows that the higher ethanol blended fuels would have a higher specific exhaust temperature $\left({ }^{\circ} \mathrm{C} / \mathrm{ft}-\mathrm{lb}\right)$ than the other fuels. This was not found when inspecting average specific exhaust temperatures measured at T/C 1 for the different fuel blends; ethanol blends actually showed lower temperatures leading to the conclusion that they actually experienced shorter burn durations. Appendix C.3 provides a more comprehensive discussion on this finding, but the implication is that it is not a suitable explanation for the observed variability.

\section{PN Summary}

Considering the information presented above, it can be said that increasing the ethanol content of the test fuel appears to increase PM emission variability, and in some cases increase PM emission levels; a similar finding was seen with the toluene blended fuels. Attempting to explain the findings from an engine behaviour point of view proved inadequate. Taking this all into consideration, the measured PM emissions appear to have been substantially influenced by changing base gasoline fuel composition, as shown by the differences between two gasoline fuelled test groups (E0 and E0 Return). Varying the aromatic fraction of the fuel as an extension of this fuel variability provided a similar response.

\subsubsection{PN Size Distribution}

Turning to the PN size distribution data shows some differences between the different fuel blends. Figure 6.15 shows the PN size distributions averaged for each fuel blend tested - the six test groups are divided amongst Figures 6.15 (a) and (b). The data presented here represents a two minute average distribution taken at the 90 minute point of a typical test to mitigate the influence of the temporal variation in PN concentrations discussed in the previous section. The error bars here indicate the standard error for the fuel blend average and serve as a marker for the run-to-run variability seen. Comparing the different fuel blends shows that the distributions all largely follow the same shape, with a dominant accumulation mode in the 70-80 nm size range. Though difficult to appreciate on these plots, the distributions also show a nucleation mode near the $10 \mathrm{~nm}$ size range, though this is markedly subdued in concentration magnitude when compared to the accumulation mode. These distributions are similar to the ones found by Mireault [44] in his previous work on this engine and in other studies in literature [61]. The large increases in PN concentrations with increasing ethanol or toluene, noted in the previous section's discussion, were manifested as increases in the accumulation mode of the distribution; the nucleation mode was affected here to a much lesser extent.

The large changes in magnitude in Figure 6.15 make it difficult to compare the different distributions shown. Normalising the distributions removes this effect and permits a better comparison. Figure 6.16 plots the distributions in Figure 6.15 normalised to the total PN concentration for each fuel blend. On a 
percentage basis it is evident that the E0 distribution is different than the other fuel blends, including the E0 Return test group. When compared to the E0 Return data, a greater proportion of nucleation mode particles and a lower proportion of accumulation mode particles were emitted. This is quite unexpected because the E0 Return test group was a repetition of the E0 test group, and has the overall effect of changing the distribution shape. Calculated modal and median ${ }^{2}$ diameters for the different distributions are contained in Table 6.2. Again, comparing the E0 and E0 Return groups shows that there was indeed a shift towards larger particles for the E0 Return group. The ethanol fuel blends also showed a shift to larger particles when compared to the E0 group, and a very slight decrease in size when compared to the E0 Return group. Increasing the ethanol content (from E10 to E30) appears to cause a slight shift towards larger particles, which is masked even in the median and modal diameter calculations. The influence of toluene is more pronounced, as a shift towards larger particles was noted. This follows available literature, which also showed size shifts to larger particles when using toluene [21].

Table 6.2: Modal and median diameters (nm) for particle size distributions for different fuel blends.

\begin{tabular}{cccc}
\hline Test Fuel & Median Diameter $^{3}$ & Median Diameter Size Bin $^{4}$ & Modal Diameter $^{5}$ \\
\hline E0 & 51.1 & 52.3 & 69.8 \\
E10 & 57.0 & 60.4 & 69.8 \\
E30 & 57.7 & 60.4 & 69.8 \\
E0 Return & 59.2 & 60.4 & 80.6 \\
T10 & 60.1 & 60.4 & 80.6 \\
T10E10 & 64.7 & 69.8 & 80.6 \\
\hline
\end{tabular}

\subsubsection{Gravimetric and PM Composition}

Inspection of the gravimetric and PM compositional results can provide some insight into the observed trends. These two off-line analyses were performed on PM laden filters collected using the methodology given in Section 5.4. As with the PN concentration and size distribution data, the concentrations (PN, mass, or compositional) reported here have been corrected for their true dilution ratios.

Gravimetric results from this investigation are presented in Figure 6.17, where they are plotted with corresponding PN concentrations measured by the EEPS during the filter collection. Though not all collected at the same time point of a test, the filters were generally collected during the back half of a typical test to mitigate the influence of the temporal drift and the initial drift associated with engine warm-up. Filter duplicates (two filters at the same time) were taken for some tests and are identified in this figure by the data point pairs at constant PN concentration. These duplicates show good agreement and were generally within the acceptable replicate filter weight variance of $15 \mu \mathrm{g}$, with the exception of one T10 run. Looking at the data here shows that PM mass concentrations increase linearly with increasing PN concentrations, indicating that the observations seen for the PN concentrations hold for the mass emissions. As with before, increasing the ethanol or the toluene fraction increased the PM mass concentrations. However, comparing the different fuel blends more closely shows that a division between

${ }^{2}$ Median Diameter is defined as the size bin where $50 \%$ of the particles are either bigger (or smaller) in size.

${ }^{3}$ Mathematical median as calculated using the above definition.

${ }^{4}$ Corresponding EEPS size bin for the median diameter. Value listed is the midpoint of the size bin, as reported by the EEPS.

${ }^{5}$ Most frequent particle size bin in the distribution histogram. 


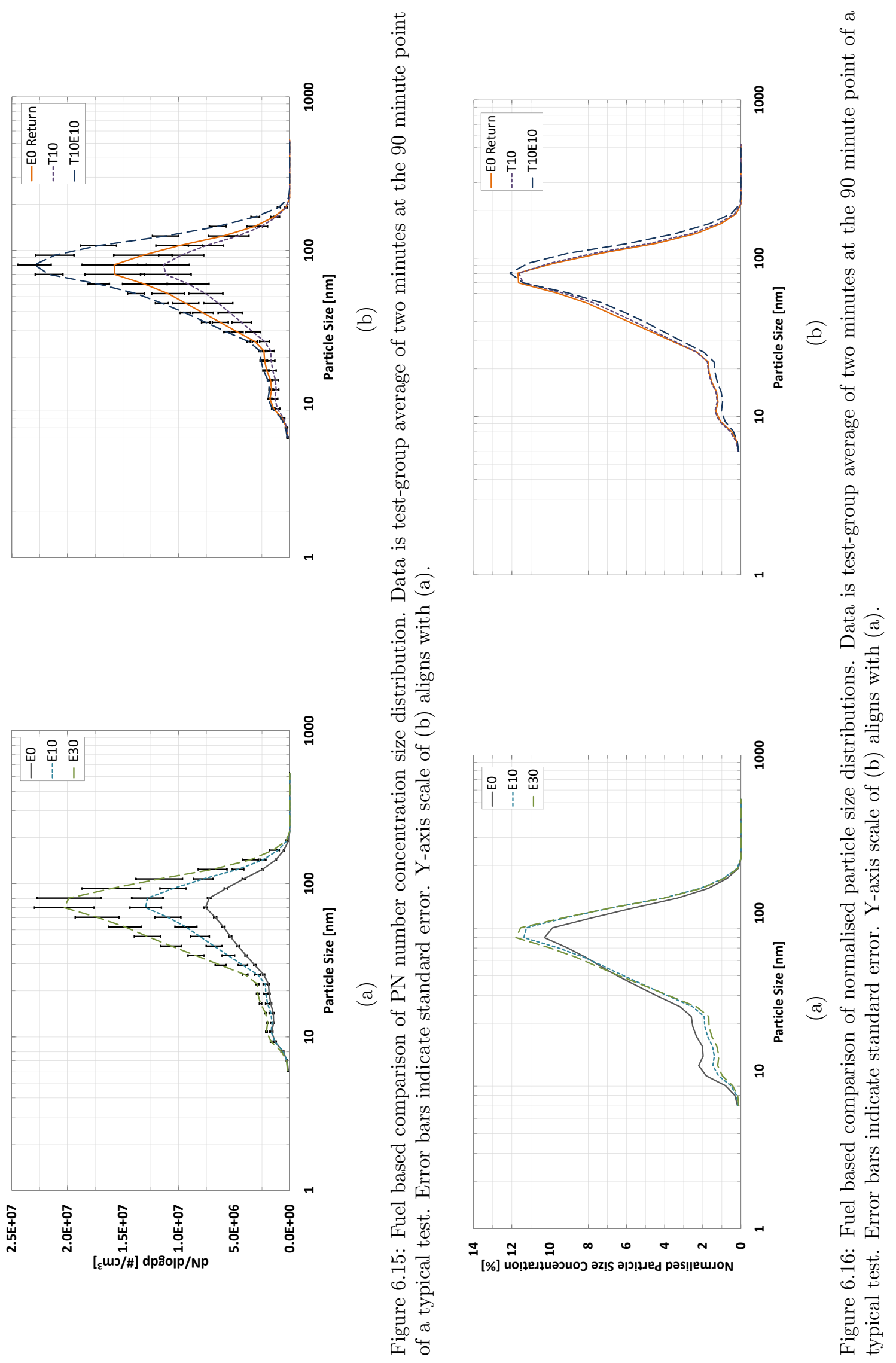


toluene and non-toluene blends occurred, with the latter emitting more mass at fixed PN concentrations. Plotting regression lines show this disparity more clearly, with the slopes of these lines giving the number of particles emitted per unit mass. Both regressions show good agreement with their respective data, indicating that the perceived difference is mathematically valid.

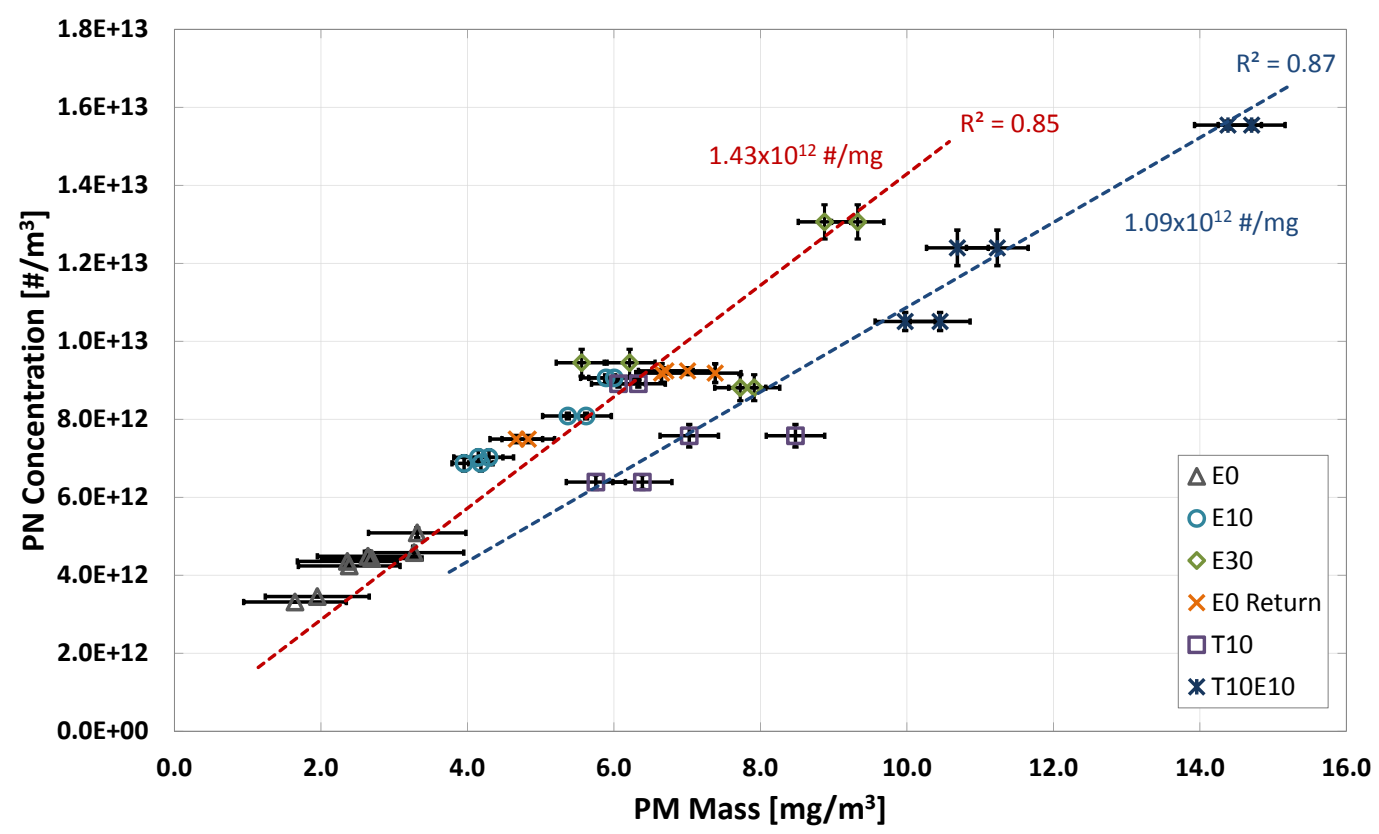

Figure 6.17: Gravimetric filter results grouped by test fuel with overlaid particle number and mass correlations. Red-dashed line includes E0, E10, E30, and E0 Return data sets; blue-dashed line includes T10 and T10E10 test groups. Error bars indicate acceptable replicate filter weight variance and one standard deviation for PN concentration for the $\mathrm{x}$ and $\mathrm{y}$ axes, respectively.

Maricq et al. [92] found the same correlation to be $\sim 2 \times 10^{12} \# / \mathrm{mg}$ for low ethanol blends which is explained as also being typical for diesel soot. While they did see an increase in their correlation for E32 blended fuel to $\sim 4 \times 10^{12} \# / \mathrm{mg}[92]$, the same was not seen in this data here. The authors reason that this indicates that the higher ethanol blends in their study produced more nuclei particles [92]. The PN size distributions presented in the previous section show that the E30 blend in this study did not produce any measurable increase in nuclei particles, so it should be expected that the correlation presented here does not shift to more particles per mass. Using the same logic actually helps to explain the observed difference for the toluene blends in Figure 6.17. It was established in the PN size distribution discussion that the toluene blended fuels (T10 and T10E10) showed shifts to larger particle distributions. Since larger particles are generally more massive than smaller ones, the observed decrease in the number to mass correlation is logical in this regard. Therefore, toluene has the additional effect of emitting disproportionately more PM mass along with the observed increase in PN concentrations.

In terms of the composition of the emitted $\mathrm{PM}$, the $\mathrm{EC} / \mathrm{OC}$ ratios in Figure 6.18 demonstrate an increasing (more elemental carbon) trend with increasing ethanol content. Comparing the two gasoline fuelled test sets shows that a higher elemental fraction was emitted for the E0 Return group than the initial E0 test, though this cannot be said with confidence given the interval overlap shown. Interestingly, the composition does not appear to have changed significantly when increasing the aromatic fraction for the T10 blend. The same cannot be said, however, for the T10E10; the additional ethanol caused a 


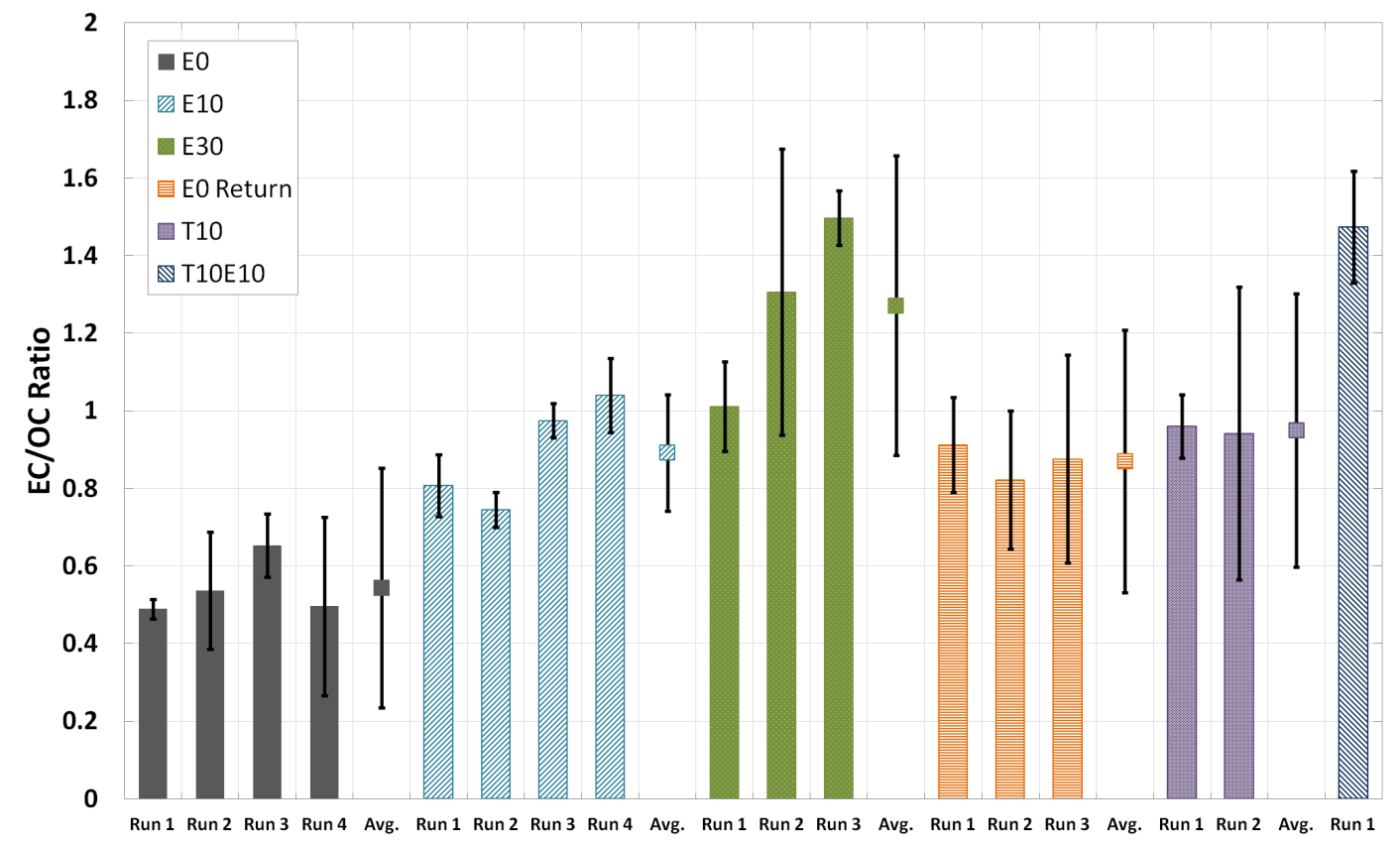

Figure 6.18: Elemental-to-organic carbon ratio (EC/OC) for the different fuel blends broken down by run and including an average for the fuel. Error bars indicate $95 \%$ confidence intervals using pooled variances.

statistically significant $(\mathrm{p}<0.05)$ increase in the elemental fraction over both gasoline test groups and the T10 group. Considering the elemental and organic carbon concentrations individually in Figure 6.19 is useful here to describe the observed trends. Returning first to the influence of ethanol, in Figure 6.19 the general increase in PM emissions (both PN concentration and PM mass) appears to be largely dictated by changes to the elemental concentration of the PM. The organic concentration in this case stays largely constant between $7-11 \mathrm{mg} / \mathrm{m}^{3}$, while the elemental concentration varied by $3-12 \mathrm{mg} / \mathrm{m}^{3}$ over the same range of ethanol (E0-E30). The same can also be said when comparing the two toluene fuel blends, where increasing the ethanol caused $44 \%$ increase in the elemental fraction and only a $13 \%$ increase in the organic portion. The conclusion is thus, quite clear; increasing the ethanol content provided a disproportionate increase in the emitted elemental carbon. This conclusion is useful in providing insight into the source of the increase in PM emissions that have been noted in a previous section, whether it be related to the combustion process or perturbations in the fuel delivery.

One hypothesis for this observation is that the increase in ethanol led to an increase in cylinder wall impingement due to vaporization difficulties. Ethanol has a high latent heat of vaporization when compared to gasoline, so at fixed injection pressures spray penetration lengths ought to increase with increasing ethanol. This can lead to wall impingement giving rise to liquid fuel burning and entrainment of lubricating oil in the fuel charge - both are known sources of soot emissions. This is difficult to directly measure, and was not done so in this investigation; however, an analogous metric of fuel dilution of the oil was proposed as marker for this wall impingement. Analysis of lubricating oil showed immeasurable quantities of ethanol in the oil; see Appendix C.4 for a more thorough discussion. Therefore, using this information alone does not confirm the validity of increasing wall impingement hypothesis. 


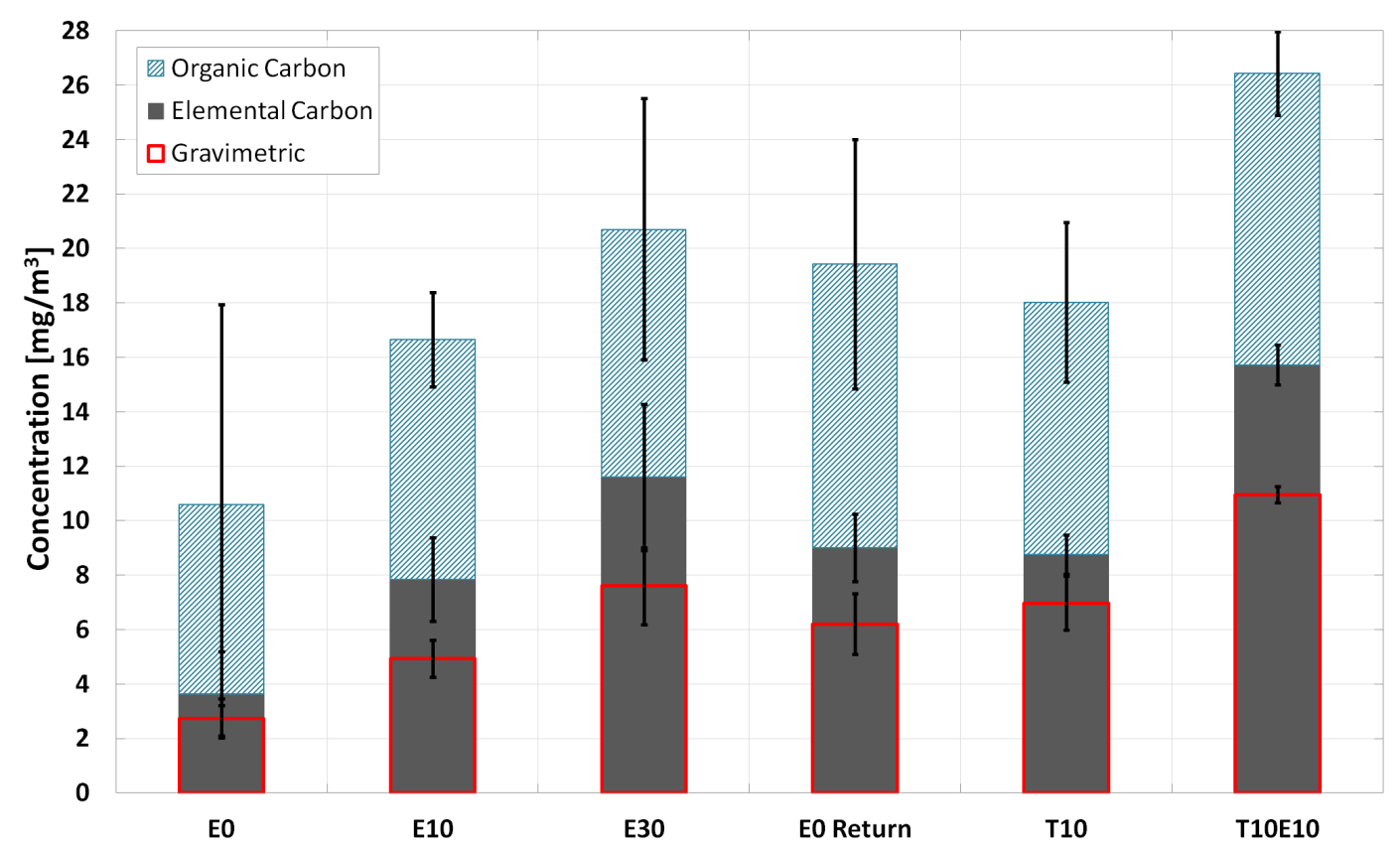

Figure 6.19: Fuel average elemental and organic carbon concentrations, with gravimetric concentrations overlaid. Error bars indicate $95 \%$ confidence intervals using pooled variances.

\section{Analysis Comparison: Gravimetric vs. EC/OC}

A digression from the above discussion; Figure 6.19 also provides useful information for comparing the measured mass concentrations using either the gravimetric or EC/OC methods. The elemental concentration should in theory provide a very similar value to the gravimetric one, as they are essentially measures of the same thing. Inspecting the figure shows that the same general trend is observed for both series and some of the test groups give good agreement, while others - chiefly E10, E0 Return, and most apparent, T10E10 - do not agree as well. Two sources of error are offered here as an explanation. The first has to do with filter processing; gravimetric filters are only weighed after 24 hours of equilibration (i.e. off-gassing), while the EC/OC analysis is performed soon after collection. The gravimetric technique is per US EPA regulations-previously discussed in Chapter 5-while the EC/OC procedure is done to mitigate evaporation of the organic fraction. It is possible that some of the heavier species that evaporate from the gravimetric filters are being pyrolized during the EC/OC analysis and end up as elemental carbon; this is supposed to be mitigated by the appropriate adjustment of the split point described previously. This brings about the second source of error offered here; that more refinement of the EC/OC processing method is required to better proportion the elemental and organic fractions. It is important to note that both of these statements are predicated on the idea that the gravimetric (US EPA) method is superior, which may or may not be the case. Regardless, given the close confidence overlap shown in Figure 6.19, this difference is likely inconsequential.

\subsubsection{Gaseous Emissions}

Gaseous emissions, including regulated and non-regulated compounds, are presented in this section. Just as before, the gaseous constituents are broken down by test group (fuel blend) and presented accordingly. Considering the gaseous emissions here may provide some further insight into the observed PM trends. 
A standard emissions bench measured regulated compounds, while the FTIR provided hydrocarbon speciation in addition to the regulated compounds. The FTIR recipe is contained in Chapter 5 for reference.

\section{Standard Emissions}

The regulated compounds measured during this investigation are presented in Figures 6.20 and 6.21 for the FTIR and emissions bench, respectively. Note that the FTIR is incapable of measuring homonuclear molecules, so $\mathrm{O}_{2}$ measurements are only shown from the emissions bench. The standard emissions results do not directly add to the discussion on PM emission variability, so a only summary of the findings is provided, including a brief comparison of instruments.

The general magnitudes of the standard gaseous emissions in Figures 6.20 and 6.21 are typical for spark-ignited engines at a slightly richer than stoichiometric equivalence ratio operating point [8]. Though not shown here, $\mathrm{NO}_{\mathrm{x}}$ emissions from this engine were found previously by Mireault [44] to be nearly entirely composed of $\mathrm{NO}$, with less then $0.1 \%$ being $\mathrm{NO}_{2}$. This bodes well with available literature on spark-ignited engines [8]. Ethanol did not have a significant $(\mathrm{p}>0.05)$ influence on the $\mathrm{NO}_{\mathrm{x}}$ emissions from this engine at this condition. This has been shown previously in other studies [44, 29] and is expected given that $\mathrm{NO}_{\mathrm{x}}$ production is highly thermally dependent (Zeldovich mechanism) and the exhaust temperatures have been shown here not to vary for the different fuels. Other studies have shown marked reductions in $\mathrm{NO}_{\mathrm{x}}$ emissions [34, 35] owing to the charge cooling effect of ethanol, but this is not seen here. The emission of $\mathrm{O}_{2}$ was also found to not deviate from expected values at slightly richer than stoichiometric condition $(\sim 1 \%)$ no matter the fuel used; an expected result because these emissions are reported as dry, removing the influence of water in the exhaust. A general trend upward of THC emissions is seen in Figure 6.21, though considering the confidence intervals shown, this cannot be said to be significant. These emissions appear to be most affected by the base gasoline (E0 vs. E0 Return) and not by the addition of ethanol or toluene.

In contrast, ethanol did have a pronounced effect on $\mathrm{CO}$ concentrations. Comparing the different fuel blends shows that both instruments measured statistically significant $(\mathrm{p}<0.05)$ decreases in $\mathrm{CO}$ concentrations with increasing ethanol content. This ethanol dependence even shows up in comparing the T10 and T10E10 runs, with the latter emitting less CO than the former. Several studies have also found this CO reduction with increasing ethanol to varying degrees [10, 29, 34, 35, 38, 39]. Wallner and Frazee [34] argue that the presence of oxygen in the ethanol molecule promotes the oxidation of CO to $\mathrm{CO}_{2}$ thereby mitigating its presence in the exhaust. This conclusion is applicable here given the strong reductions in $\mathrm{CO}$ output observed.

Water emissions appear to have increased with increasing ethanol content as measured by the FTIR; the calculation in the emissions bench data also shows this increase. Again, this trend also holds for the toluene fuels (T10E10 compared to T10) and is logical given that ethanol has a H:C ratio of 3, while gasoline is much closer to 2 - assumed here to be 1.85 per recommendations of the US EPA for E0 [79]. By the same token, including toluene in the fuel blend caused a decrease in exhaust water concentrations due to its lower H:C ratio of 1.78. Mireault [44] found a similar result, though was cautious to attribute this to ethanol because the intake air humidity was not accounted for and could be confounding the results. In this investigation, test cell air humidity was found to not vary appreciably due to the HVAC control of the room, something which was not available during Mireault's work. So it is probable that the observed water trend is intrinsic to the ethanol as postulated. 


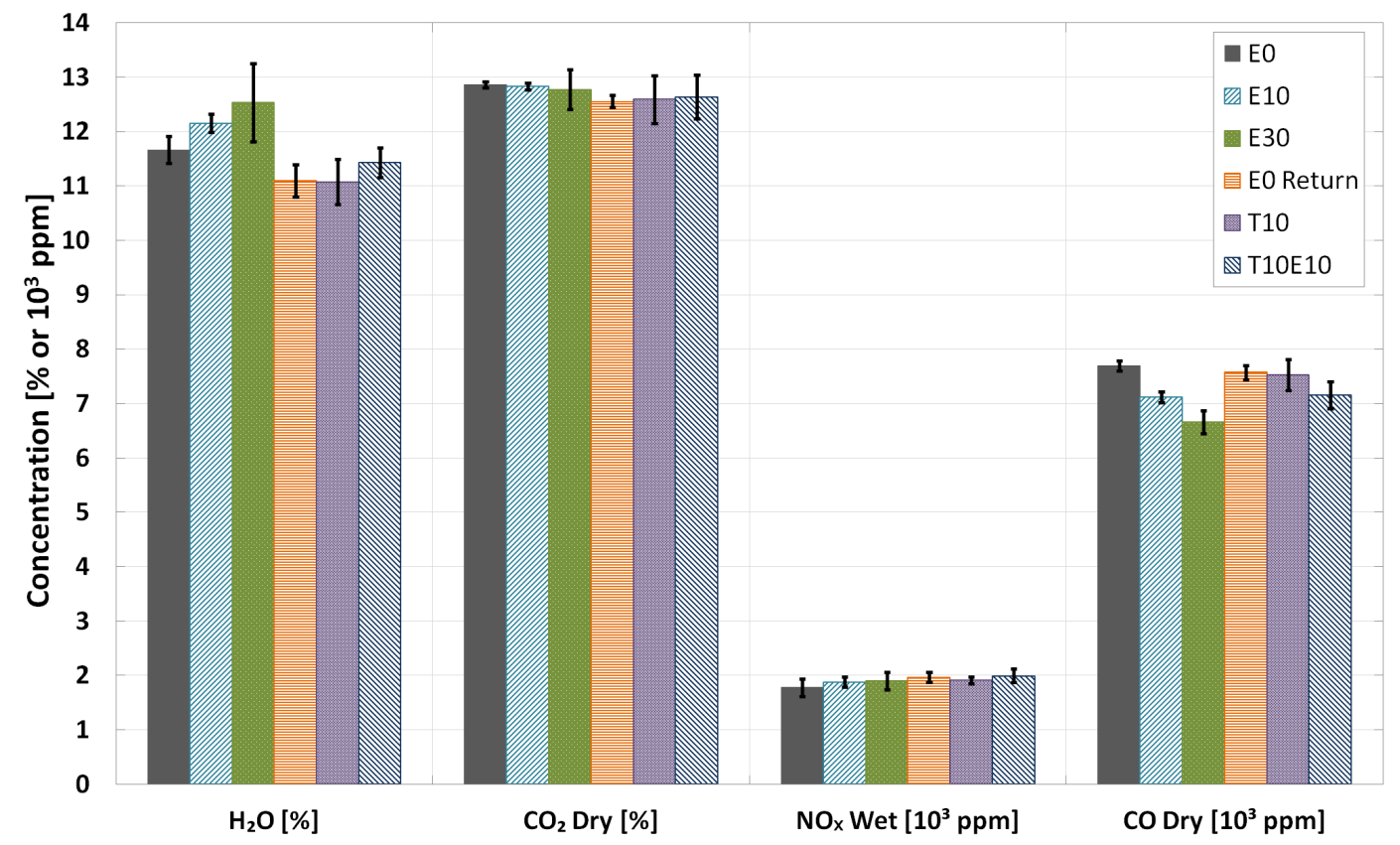

Figure 6.20: Average standard emissions measured by the FTIR for the different test fuels. $\mathrm{CO}_{2}$ and $\mathrm{CO}$ emissions are reported as dry, using measured $\mathrm{H}_{2} \mathrm{O}$ content. Error bars indicate $95 \%$ confidence intervals.

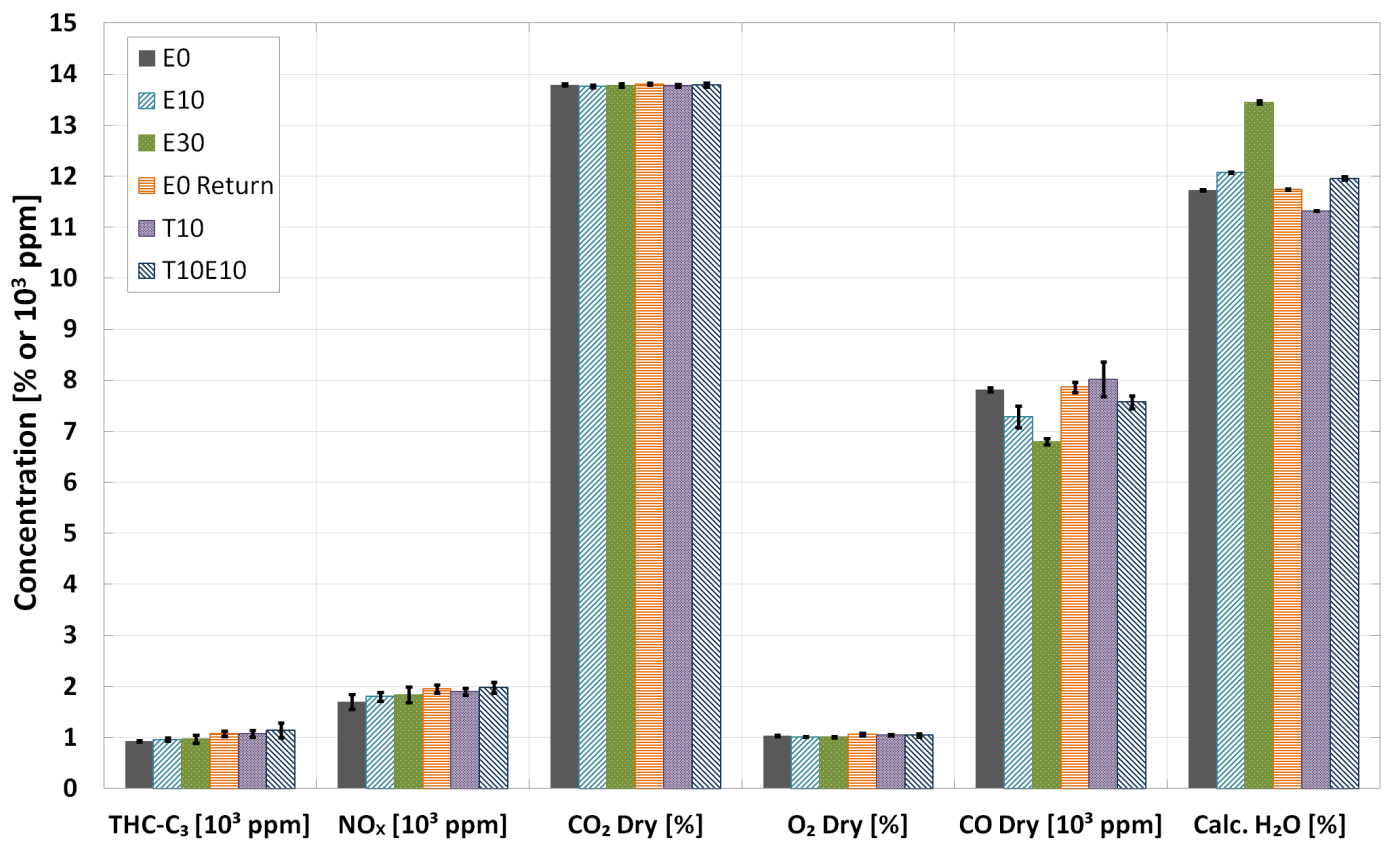

Figure 6.21: Average standard emissions measured by the emissions bench for the different test fuels. Emissions are reported as measured wet for THC and $\mathrm{NO}_{\mathrm{x}}$, and dry for $\mathrm{CO}_{2}, \mathrm{O}_{2}$, and CO. Calculated $\mathrm{H}_{2} \mathrm{O}$ and corrected $\mathrm{O}_{2}$ are based on method presented in Appendix B.2. Error bars indicate $95 \%$ confidence intervals.

\section{Instrument Comparison: FTIR vs. Emissions Bench}

Examining the data shown in Figures 6.20 and 6.21 shows some differences exist between the measurements produced by the FTIR and the emissions bench. A summary of this comparison is provided 
here; Appendix C.5 details a more thorough discussion for reference. Regardless of fuel, $\mathrm{CO}_{2}$ and $\mathrm{CO}$ measurements from the emissions bench were consistently higher than FTIR concentrations by $7 \%$ and $\sim 2 \%$, respectively; $\mathrm{NO}_{\mathrm{x}}$ measurements showed the opposite, where concentrations were consistently lower by $\sim 5 \%$. FTIR measured water concentrations were noted to be slightly lower than the calculated values using the method described in Appendix B.2. Correcting the FTIR concentrations for this calculated water content yielded no appreciable improvement. Turning to the literature provides some insight; Wallner and Frazee [34] performed a similar instrument comparison and found similar differences for the above compounds $( \pm 5 \%)$. It should be pointed out that previous work on this apparatus found these differences to be much higher at approximately 10-15\% depending on the species [44]. The changes here can be explained by the fact that some improvements to the ranging of the emissions bench were performed prior to this investigation, along with the use of newer calibration gas mixtures. Therefore, given the information from literature, the current differences appear acceptable and bode well for the use of the FTIR as gaseous measurement instrumentation.

\section{FTIR Speciation}

Using the FTIR to provide a hydrocarbon speciation of the gaseous constituents in the exhaust stream provides a unique opportunity to correlate the PM emissions behaviour to certain gas phase compounds. These FTIR results are contained in Figures 6.22 and 6.23 and are averaged for the different fuel blends tested. The error bars again show $95 \%$ confidence intervals and are used here to determine the statistical significance of the results.

Aldehyde emissions have been linked to ethanol combustion in several previous studies, where increasing the ethanol content of the fuel led to increases in measured aldehyde emissions [29, 33, 34, 35, 36, 37]. The results from this engine are no different in this regard; both formaldehyde and acetaldehyde emissions increased with increasing ethanol content. Acetaldhyde is an intermediate species in the combustion of ethanol, and is readily formed via hydrogen abstraction (H-abstraction) of the ethanol molecule [93]; formaldehyde can also form from the breakage of the $\mathrm{C}-\mathrm{C}$ bond in the initial step of ethanol combustion, though this is not observed as major intermediate species [93]. Inspection of Figure 6.22 demonstrates significant increases $(\mathrm{p}<0.05)$ in acetaldehyde emissions for the E10 fuel blend, and further increases with the E30 fuel when compared to the two gasoline data sets (E0 and E0 Return). This observation again holds true for the toluene blended fuels, with T10E10 emitting more than T10. With regards to formaldehyde, the increase is less certain; only the E30 test group showed statistically higher emissions at the $95 \%$ confidence level when compared to the other fuels. This follows the assertion that formaldehyde production is largely independent of ethanol fraction, especially at these low levels - a conclusion also reached by other studies $[29,35,36]$.

In both figures presented here, running E30 resulted in a reduction of measured gaseous species of three or more carbon atoms (i.e. isobutylene, 1,3 butadiene, propylene, and pentane); this effect was not generally seen with E10. This result is likely due to the displacive effect that follows with mixing ethanol with gasoline; the higher order hydrocarbons are reduced in quantity so it follows that the intermediate species from the combustion of these compounds are also reduced. Other compounds that show trends with increased ethanol and toluene fractions are exactly those species - ethanol and toluene. While this may seem intuitive and expected, the implication of this is rather important; the presence of these species in the exhaust can be correlated back to the overall fuel composition. Indeed, Kar et al. [33] saw increasing species emission with increasing species fuel mass fractions for different constituents, which 


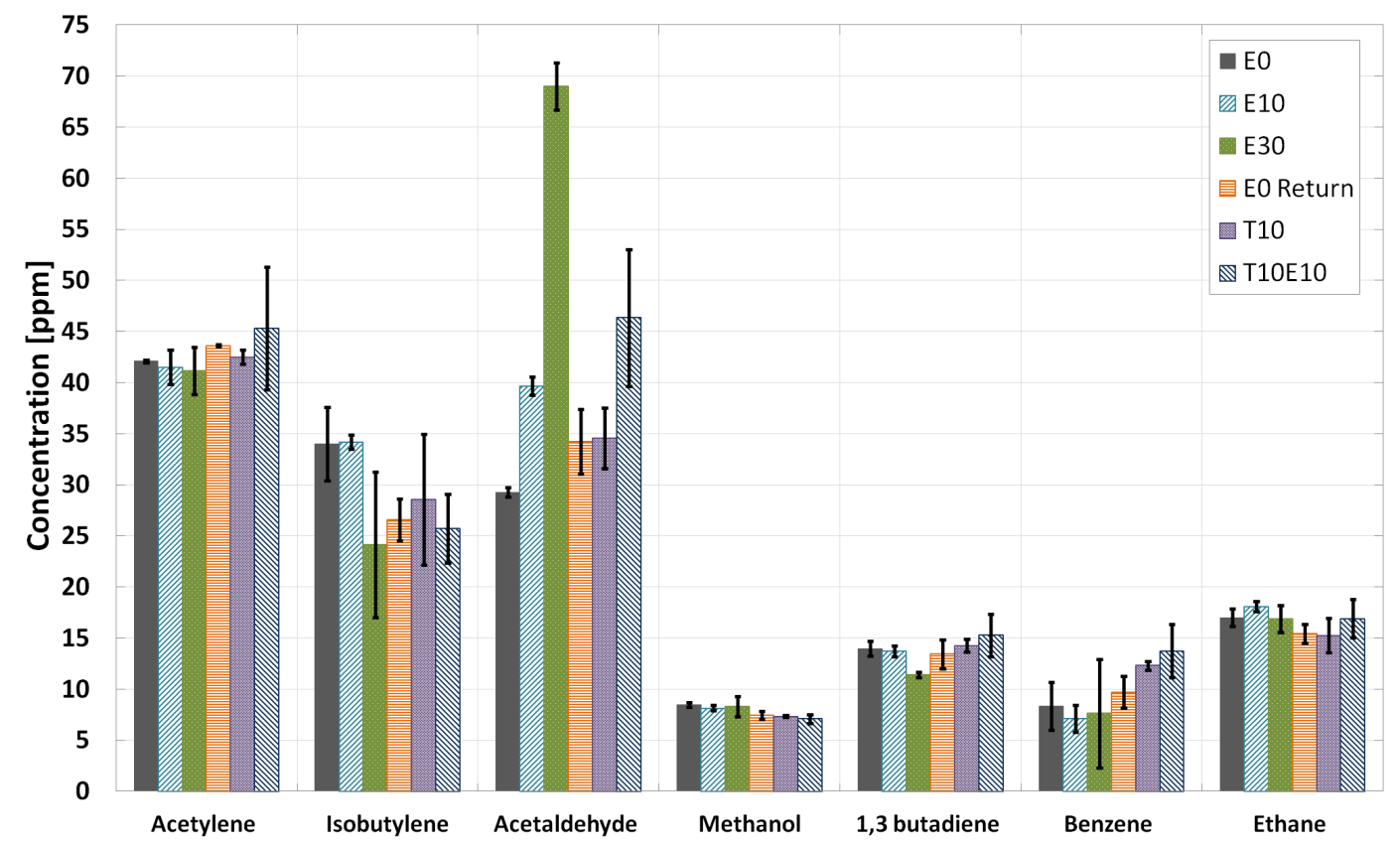

Figure 6.22: Average hydrocarbon emissions measured by the FTIR for the different test fuels. Error bars indicate $95 \%$ confidence intervals.

corroborates the above observation in this work. Of course the production of intermediate species during combustion is an important consideration here, especially in discussions regarding PM production, but the implication of this concept is that measurements from the FTIR can be used to make inferences on fuel composition. As further evidence to this, comparing the two 10\% ethanol blends (E10 and T10E10) shows that even with the additional toluene, ethanol emission levels remained constant and at levels seen previously on this engine for E10 [44].

Returning to the question of PM formation: ethylene and acetylene are fundamental for the discussion on PM emissions due to their ability to form unsaturated polyacetylenes from the H-abstraction acetylene-addition (HACA) mechanism. These polyacetylenes are considered a major precursor to soot production, along with PAHs [8]. Review of Figure 6.23 demonstrates that ethylene showed only modest changes in concentration depending on the fuel composition; acetylene production appears to have been largely independent of fuel composition according to Figure 6.22. The fact that these two compounds, which are important for PM production, did not vary in significant quantities implies that the observed PM response with varying fuel composition did not come about from these pathways. Instead, aromatics that can decompose to form PAHs from dehydrogenation and recombination likely played a more influential role in the observed variability. Figure 6.22 shows that some changes in benzene concentrations occurred when comparing the different fuel blends, though this cannot be said with confidence at the $95 \%$ level. Turning to the other aromatic compound measured here paints a different picture; measured toluene concentrations in Figure 6.23 did exhibit statistically significant $(\mathrm{p}<0.05)$ changes with changing fuel composition. Not surprisingly, the purposely added toluene in the toluene blended fuels (T10 and T10E10) produced the highest concentrations of toluene emissions, which again follows the conclusion of Kar et al. [33]. However, comparing the two gasoline test groups (E0 and E0 Return) shows almost a 50\% increase in measured toluene concentrations. This is peculiar because the fuel blend was nominally the same in both situations; i.e. same fuel supplier and no ethanol or toluene purposely added. The concern 


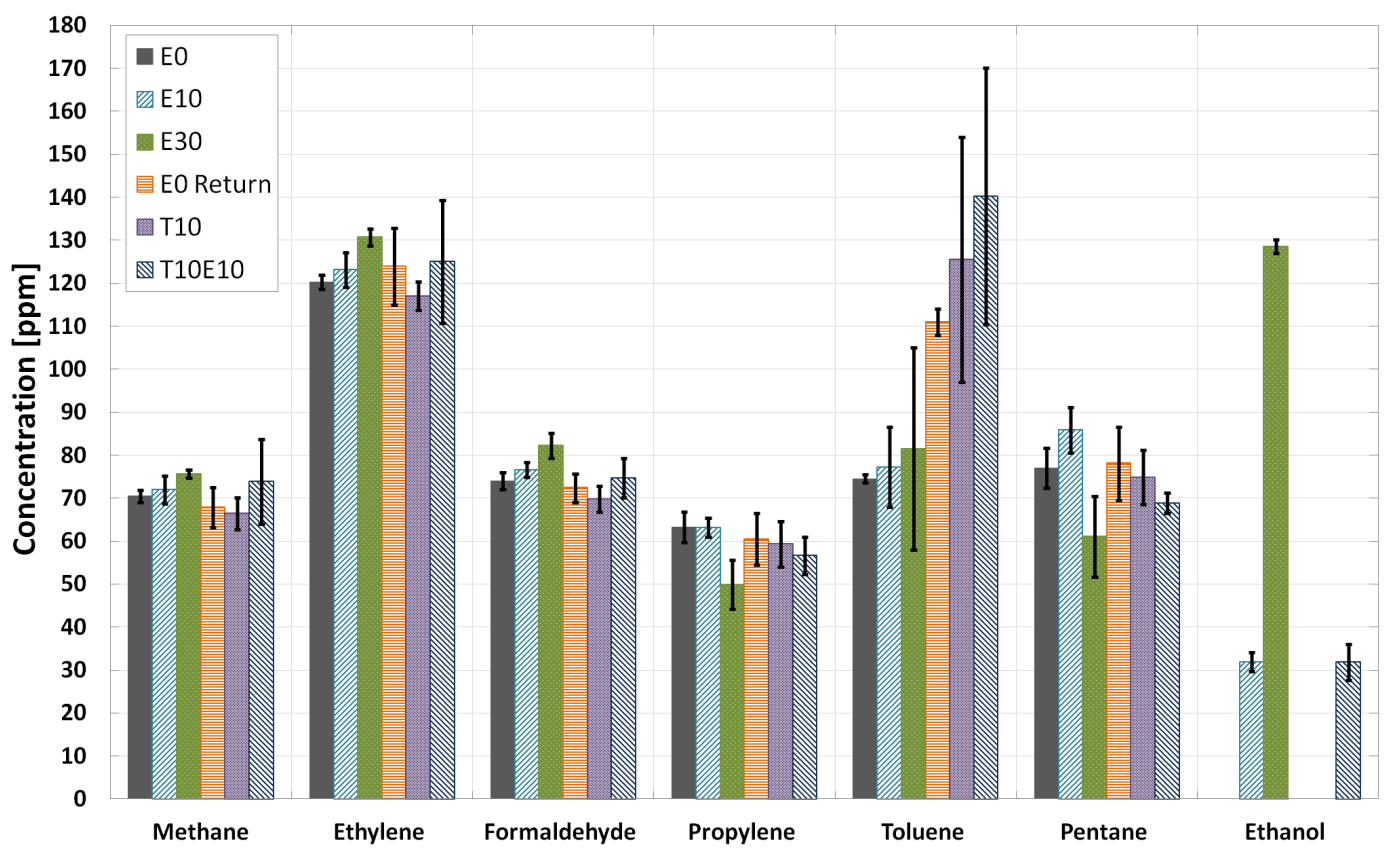

Figure 6.23: Average hydrocarbon emissions measured by the FTIR for the different test fuels. Error bars indicate $95 \%$ confidence intervals.

here is that a substantial change in base fuel composition had occurred and as a result, was the main driving force behind the observed changes in PN concentrations noted in Section 6.2.1 for these two test groups in question. This has further implications for the other fuel blends because changes to the base gasoline could have propagated to their overall composition as well. This puts into question whether fuel compositional changes at the base level were really behind all the variability noted, especially when considering the error bars for the toluene data in Figure 6.23 trend well with the standard error of the PN averages in Figure 6.13. Considering the remaining compounds presented in these two figures, only isobutylene demonstrates a substantial and statistically significant difference in concentration for the two gasoline fuelled test groups, and it shows substantial variability with the other fuel blends.

\subsection{PN vs. Gaseous Species}

The preceding section established the presence of a pronounced fuel dependence on PM emission during this investigation. Through analysis of gaseous emissions data, several gas phase compounds that displayed large changes in exhaust concentration were identified. Using the assumption that differences in exhaust concentrations of specific species equates to differences in fuel composition, it was postulated that perhaps these gaseous components could be used as markers for the observed PM emission variability. In order to judge the validity of this hypothesis, the measured PN concentrations were regressed with the selected gaseous phase compounds independently; the results from that analysis work are presented in this section. Three compounds were selected on the basis of their observed behaviour; ethanol, toluene, and isobutylene. All three species appeared to visually trend with either changes in magnitude of the PN concentrations or the observed emissions variability.

Linear least-squares (LLS) regressions were performed for both toluene and isobutylene against PN 
concentrations on an individual basis; ethanol was not directly regressed because measured concentrations behaved in a step-wise fashion depending on the fuel blend. Instead, regressions were performed at constant ethanol levels by segregating the data based on the amount of ethanol in the fuel blend. Note that Fuel A data - corresponding to data presented in the systematic sources discussion (Section 6.1) - is included here and was previously found to contain ethanol approximately equivalent to a $10 \%$ by volume fuel blend based on measured exhaust concentrations. Additionally, the data points with shaded marker faces were obtained during the "Fuel Supplier Switch" test group (see Table 6.1 for test matrix); they have not been included in the E0 and E10 groups presented elsewhere, but are included here for reference. A regression for E30 was not performed due to the limited number of data points available; however, the data is presented on these plots for visual comparison. PN concentrations were obtained at run-end to mitigate the impact of the temporal variations (i.e. drift) on the regressions.

\subsubsection{Toluene}

The correlation of measured toluene exhaust concentrations to PN concentrations is presented in Figure 6.24. In general, both regressions show good agreement with their respective data, as shown by the coefficients of determination. This is an indication that the perceived influence of toluene on PM emissions is in fact valid. In both cases, PN concentrations generally climb as the toluene exhaust concentrations increase, while increasing the ethanol content to $10 \%$ also shifts PM emissions upward, as was previously noted. The fact that both regression lines show similar slopes implies that this effect is equally applied at varying concentrations of toluene. However, there still appears to be some scattering of the data at constant toluene concentrations, seen most evidently in the Fuel A data group. Here toluene emissions did not vary by more than approximately $20 \%$, yet measured PN concentrations varied by almost $90 \%$. Inspection of ethanol emissions for this group shows negligible variance within statistical significance, thereby implying that some other factor was at play. The three shaded triangles and the E0 Return group provide a similar observation at the $0 \%$ ethanol level, where increases in PN concentrations occurred at more or less constant toluene exhaust concentrations. This group will be important for the identification of the missing factor.

\subsubsection{Isobutylene}

Regressing the PN concentrations with isobutylene provides a pathway for this missing factor. Figure 6.25 demonstrates the correlation of PN concentrations with isobutylene; increasing concentrations of isoutylene coincide with reductions in PM emissions. Again, both regression lines show acceptable agreement with the data, giving mathematical credence to this co-dependence at both fixed ethanol levels. Recall that the three shaded triangles and E0 Return test group in Figure 6.24 showed increases at fixed toluene concentrations. Here, the same two groups of data points show that this coincides with changes in measured isobutylene concentrations. Furthermore, the Fuel A data points are now aligned with decreasing isobutylene levels. In this case, the observed scatter serves as an indication for the variability in measured toluene concentrations in Figure 6.24, and vice versa. As a final indication for this observation, inspecting the E10 data series (open circles) shows in Figure 6.25 that isobutylene concentrations did not vary substantially; those changes in PN concentrations are therefore a result in changes to the toluene fraction, as shown in Figure 6.24. 


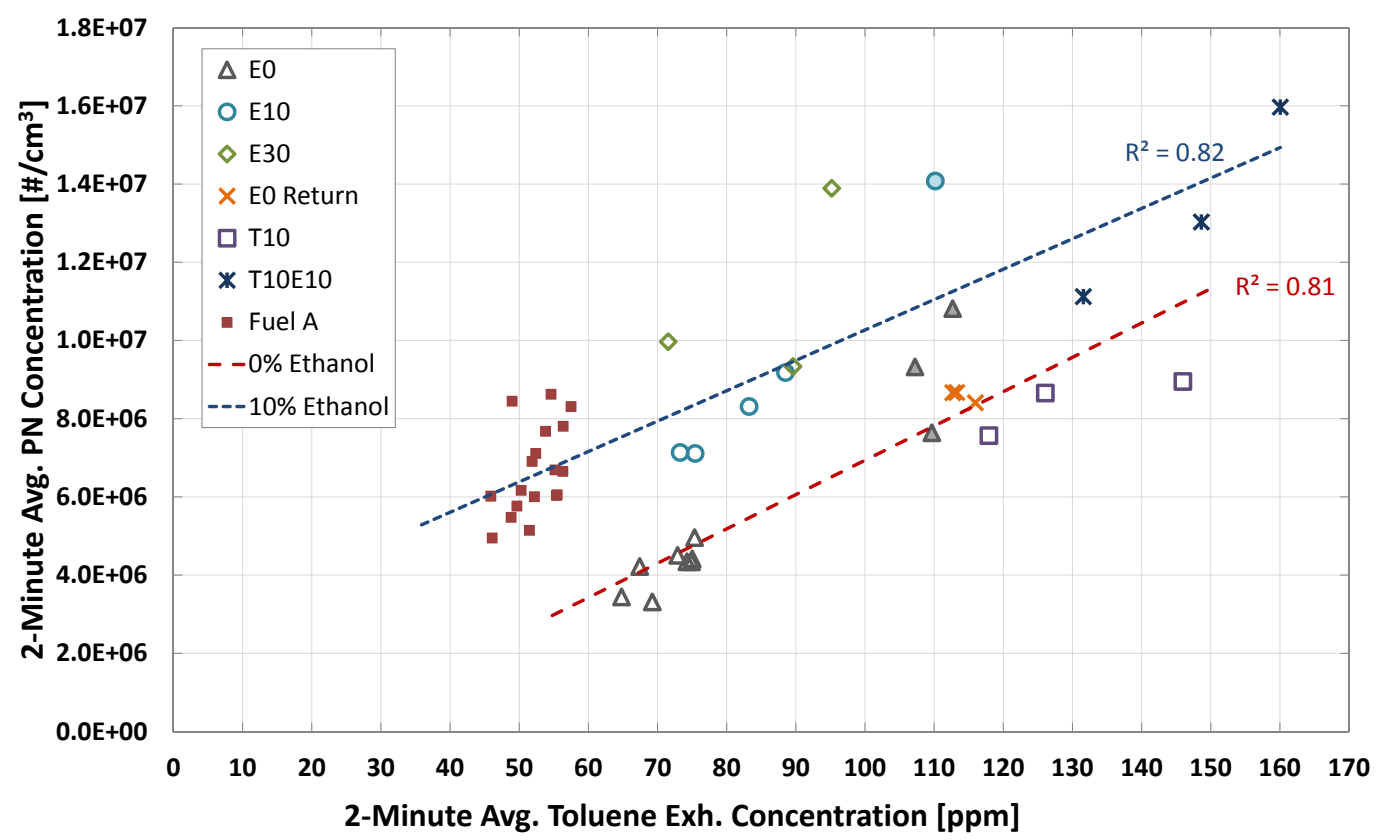

Figure 6.24: Two-minute average PN vs. toluene at run end for the different test fuels with LLS regression lines. 0\% Ethanol regression includes E0, E0 Return, and T10 data points; 10\% Ethanol regression includes E10, T10E10, and Fuel A data points. Shaded marker faces are from Fuel Supplier Switch test group in Table 6.1.

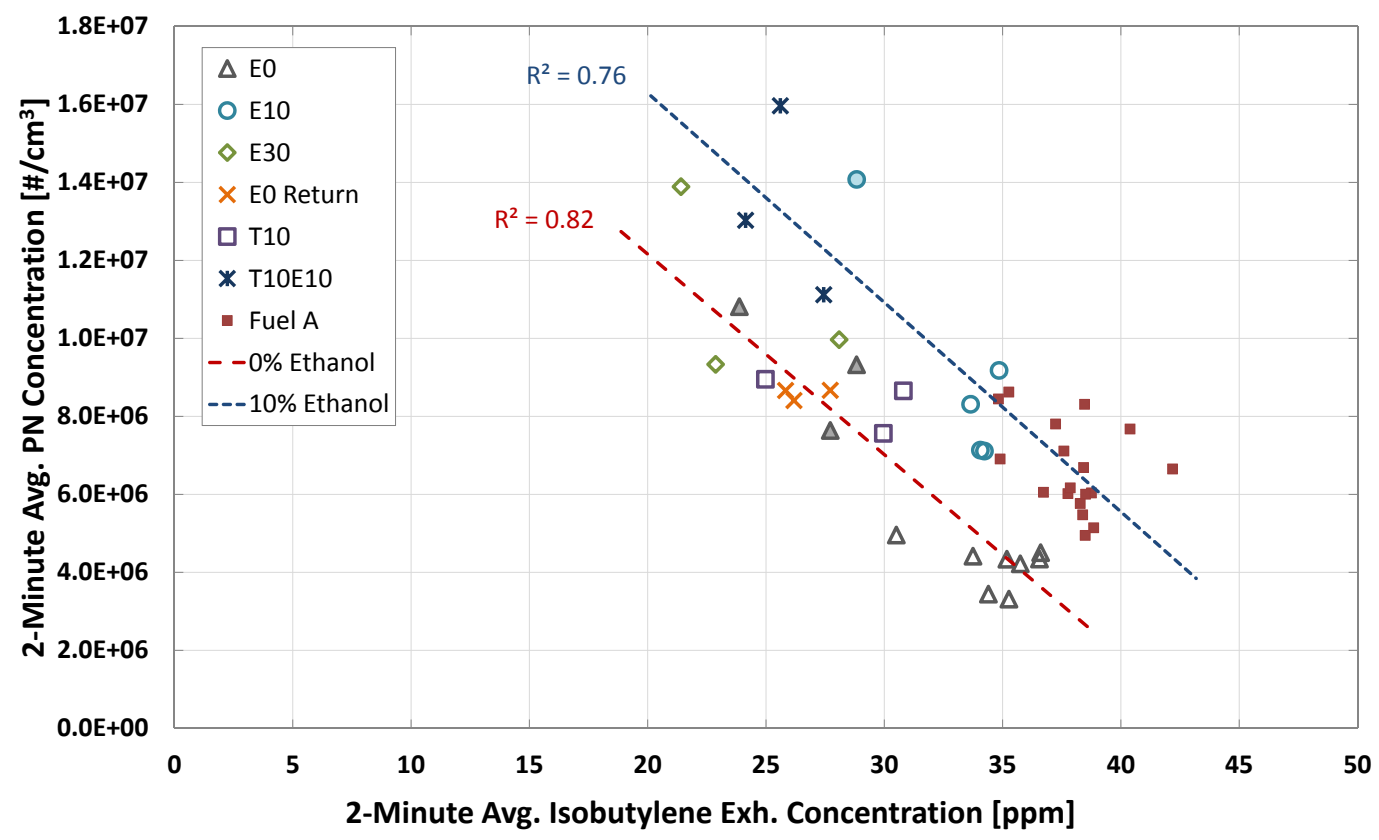

Figure 6.25: Two-minute average PN vs. isobutylene at run end for the different test fuels with LLS regression lines. 0\% Ethanol regression includes E0, E0 Return, and T10 data points; 10\% Ethanol regression includes E10, T10E10, and Fuel A data points. Shaded marker faces are from Fuel Supplier Switch test group in Table 6.1. 


\subsubsection{Multiple Linear Regression}

The combination of these two regressions provides some insight to the observed variability on three levels: (1) increasing the ethanol fraction provides a step upwards in measured PN concentrations; (2) increasing the toluene fraction of the fuel provides for increasing PM emissions; and (3) the observed scatter of PN concentrations at fixed toluene and ethanol levels can be effectively explained by changes in the isobutylene emissions. Note that observations (2) and (3) can be reversed and are interchangeable in that regard; changes in toluene can also explain the scatter of PN concentrations at fixed isobutylene emissions levels.

As a final point of analysis, the three gaseous compounds were regressed with measured PN concentrations in a multiple linear regression (MLR). This in effect creates a predictive model of PN concentrations, though this does not serve purpose in this discussion here. Instead, this MLR also provides confirmation that the three influences cover the observed fuel compositional dependence well as a combined effect. When combined together, the resulting regression (Equation $6.1^{6}$ ) provided an excellent fit of the data shown by an adjusted coefficient of determination of 0.85 . Further, all the coefficients given in Equation 6.1 were determined to be statistically significant $(\mathrm{p}<0.01)$, implying that they are relevant for explaining the changes in measured PN concentrations.

$$
\text { PNConc. }\left(\# / \mathrm{cm}^{3}\right)=1.06 E 7+4.37 E 4 \times[\text { Toluene }]+1.19 E 5 \times[\text { Ethanol }]-2.67 E 5 \times[\text { Isobutylene }]
$$

\subsection{PN Index and Varying Fuel Properties}

The individual LLS and MLR analysis work presented above indicates that the PM emissions correlate to changes in the three compounds. However, this analysis has thus far provided no physical meaning behind these results, nor has it assessed the appropriateness of such a finding - i.e. Why these specific compounds? In this cumulative section of this chapter the observed trends are explained from a physicochemical perspective.

First it is necessary to put the above results in some context, and for that some literature presented in Chapter 3 will need to be recalled. The works of Aikawa et al. [63] and Leach et al. [64] both provided PM emissions indices based on two metrics of fuel composition; the vapour pressure and the weighted DBE (double-bond equivalent) summed for the different constituents of the fuel. Their independently derived indices are presented in Equations 3.1 and 3.2 in Chapter 3. As previously discussed, the DBE value of a particular fuel constituent serves as a metric for its sooting propensity, while the vapour pressure describes how readily the fuel will evaporate and mix with the air [63,64]. As may be expected, these indices are proportional to the fuel's DBE score and inversely proportional to its vapour pressure (DVPE). Using a simplified methodology (see Appendix C.6) based on the method provided by Leach et al. [64], PN index values were calculated for the five major test blends used during the fuel composition portion of this investigation: E0, E10, E30, T10, T10E10. These values in Table 6.3 demonstrate the observed PM emission behaviour in a general sense; increasing the ethanol and toluene fractions give rise to increased PN indices due to changes in fuel chemistry and physical properties. Additionally, the index provides insight into the observed trends. As an example, the addition of ethanol changes the chemistry of the fuel to lower sooting propensity as shown by the DBE score. However, this is offset to a greater extent by the reduction in vapour pressure leading to an overall higher PN index score. This

${ }_{6}^{6}[$ ] denotes measured species exhaust concentration in ppm 
result at the index level follows those shown previously at the PM emissions level.

Unfortunately, the indices do not accurately describe the variability in the emissions seen during some of the test groups, nor the observed differences between similarly fuelled test groups (i.e. E0 and E0 Return). To explain these scenarios one must accept that changes to the base fuel composition occurred.

Table 6.3: Calculated PN emissions index using a variation on the procedure from Leach et al. [64]

\begin{tabular}{cccc}
\hline Test Fuel & DBE+1 & DVPE $(\mathrm{kPa})$ & PN Index \\
\hline E0 & 2.6 & 53.78 & 4.83 \\
E10 & 2.44 & 46.0 & 5.31 \\
E30 & 2.12 & 34.8 & 6.09 \\
T10 & 2.84 & 48.0 & 5.92 \\
T10E10 & 2.68 & 41.0 & 6.53 \\
\hline
\end{tabular}

Extending this discussion to the remaining compounds (toluene and isobutylene) allows for explanations to begin to be formed. From the regressions performed in the last section, toluene was shown to be strongly correlated to the measured PN concentrations and likewise variable at nominally fixed fuel blend composition. Toluene exhibits a high DBE value so it is logical that the PN indices should be higher for runs with greater toluene concentrations in the exhaust. However, to explain the importance of the correlation with isobutylene requires a digression into gasoline fuel composition and combustion, provided in the next sub-section.

\subsubsection{Variations of Base Gasoline}

Changes in the base gasoline composition have been alluded to in several of the preceding sections without much further comment. Indeed, changes in measured toluene and isobutylene exhaust concentrations at nominally fixed fuel blends seem to indicate that perhaps base fuel changes occurred during this investigation.

Gasoline is a multicomponent fuel, comprised of many different hydrocarbons ranging from 4-12 carbon atoms in size (C4-C12). Through crude oil distillation and other refining processes, extracted gasoline is composed of a mixture of parafins, olefins, cycloparafins, and aromatics [94, 95]. Olefin and aromatic fractions are typically limited by regulations to approximately $10 \%$ and $35 \%$ by volume, respectively, while saturates make up the remainder [95]. Within this framework, commercial gasoline typically varies by not only the octane rating, supplier, and the crude oil source, but also throughout the calendar year due to changes in vapour pressure requirements ${ }^{7}[94,96]$. In order to meet these compositional and physical property guidelines, gasoline refineries modify the overall fuel formula to suit. For example, to boost the vapour pressure during cold weather (i.e. lower the overall boiling point of the fuel), lighter and more volatile components will be included while heavier components might be removed; the converse is done in warm months.

To illustrate this effect in this investigation requires a return to FTIR data. Figures 6.26 and 6.27 present the speciated hydrocarbon emissions for the two base gasolines used in this investigation (Fuel

${ }^{7}$ Vapour pressure — typically measured as Reid Vapour Pressure (RVP)—is controlled in the summer to mitigate evaporative emissions. 
A and Fuel B), except now organized by the month in which the tests were taken. This is done to highlight the seasonal variation of the fuel, which is readily demonstrated in both figures. For example, in both figures lighter olefins - such as ethylene, propylene, and isobutylene - are shown to decrease in concentration with the progression from late winter/early spring to the summer. These olefins are readily formed during combustion of lighter alkanes (C4-C8) as intermediate species through H-abstraction and $\beta$-scission mechanisms [93, 97].

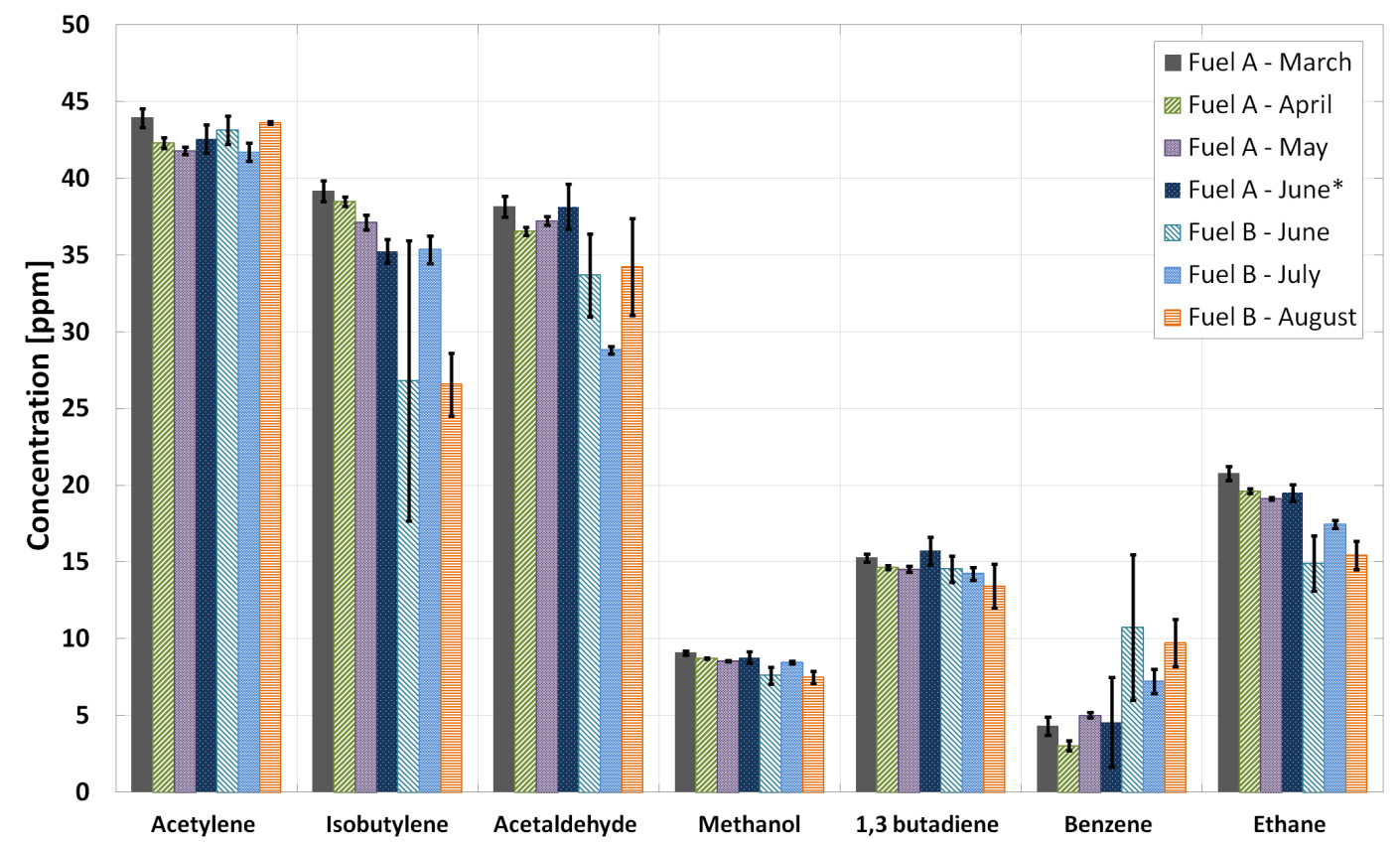

Figure 6.26: Average standard emissions measured by the FTIR for the two base gasolines (Fuel A and Fuel B). Data series with * represents a single run. Error bars indicate $95 \%$ confidence intervals or one standard deviation*.

These figures also provide some insight into the selection of isobutylene as the marker for vapour pressure. Comparing these three compounds, isobutylene underwent the largest percentage change through the progression of seasons; $32 \%$ vs. $20 \%$ and $12 \%$ for propylene and ethylene, respectively. Additionally, ethylene is likely confounded by the fact that it is readily formed by many of the typical constituents in gasoline, including ethanol. Finally, though not presented in this work, isobutylene provided the strongest correlation to measured PN concentrations of these three compounds, lending additional credence to its use as a marker. The suitability of isobutylene as a marker for vapour pressure by extension of lighter hydrocarbon combustion can therefore be considered both theoretically valid and corroborated by emissions data.

Considering just Fuel B confirms the main culprit for the observed PM emissions variability as being fuel compositional changes. In Figures 6.26 and 6.27, Fuel B - July and Fuel B - August actually correspond with the E0 and E0 Return test groups, respectively. Comparing these two groups shows once again that statistically significant $(\mathrm{p}<0.05)$ increases in toluene $(\mathrm{DBE})$ and decreases in isobutylene (vapour pressure) occurred; however, their effect on PM emissions is now made clear with the use of the PN index. Adding further credence to this is the apparent variability in these measured emissions giving rise to the variability in PM emissions presented in Section 6.2 and confirmed in the regressions in Section 6.3. Therefore, given the information presented it is likely that vapour pressure and DBE 
changes to the base gasoline occurred during the fuel composition portion of this investigation. These changes at the base level are likely present in the results of the other fuel blends, given the variances seen and the performed correlations.

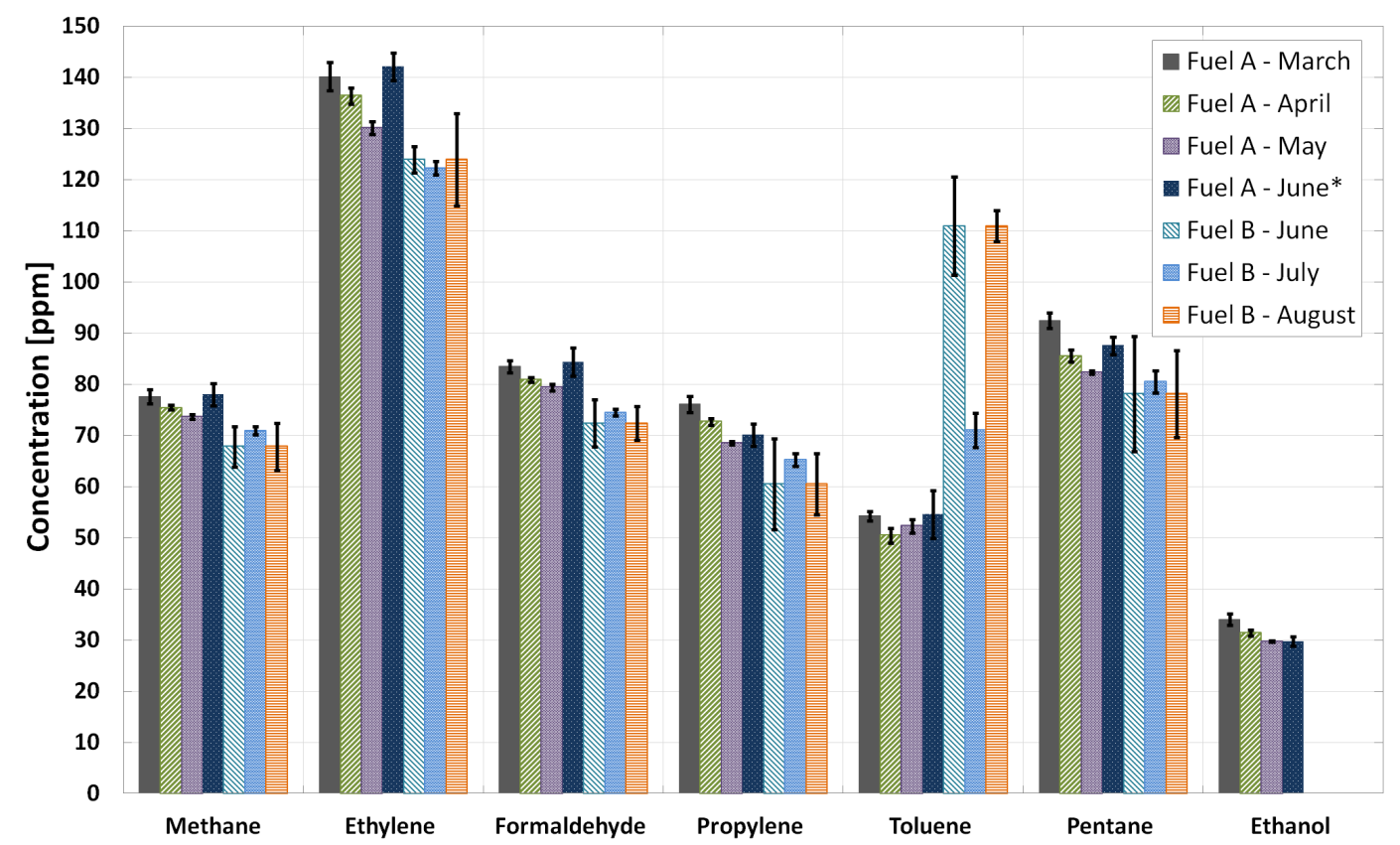

Figure 6.27: Average standard emissions measured by the FTIR for the two base gasolines (Fuel A and Fuel B). Data series with * represents a single run. Error bars indicate $95 \%$ confidence intervals or one standard deviation*.

\subsubsection{PN Index Revised}

As shown in Table 6.3, the PN index as it stands insufficiently describes the observed PM emissions in this investigation. However, this statement should not be construed as deprecating the index as a whole. The problem here stems from the fact that the PN index requires the composition of the fuel, in terms of DBE and vapour pressure, to be known. A study using commercial fuels without an accompanying fuel analysis will be prone to error from uncertainties.

In any case, using the measured toluene and isobutylene concentrations from the FTIR, one can make adjustments to the PN index to better suit the data, at least to a first approximation. Appendix C.7 details the process undertaken to provide a corrected emissions index. In general terms, runs with higher isobutylene concentrations had the vapour pressure of the base gasoline fraction artificially increased, while runs with higher toluene concentrations had their DBE scores artificially increased. Figure 6.28 plots the revised PN index with the average measured PN concentrations for the various test groups performed; the PN indices listed in Table 6.3 are included for comparison. The corrected index shown in Figure 6.28 demonstrates the behaviour of the PM emissions from the various fuel blends better than initial index. However, there are some shortcomings with this correction method which cannot be overlooked. For example, this correction assumes that toluene and isobutylene emissions are not displaced by the addition of ethanol or toluene in the fuel blending process. To overcome this error, changes to the original index were only applied to the base gasoline; however, the extent of changes 
to gasoline is difficult to determine when considering exhaust emissions from a fuel blend. All factors considered, what the revised index demonstrates is that these two factors, DBE and vapour pressure, can be retroactively used to make sense of the observed PM emissions, as has been done here. Furthermore, the true value of this correction process lies in the fact that it can help guide future fuel based studies to appropriately account for the two major fuel predictors of PM production.

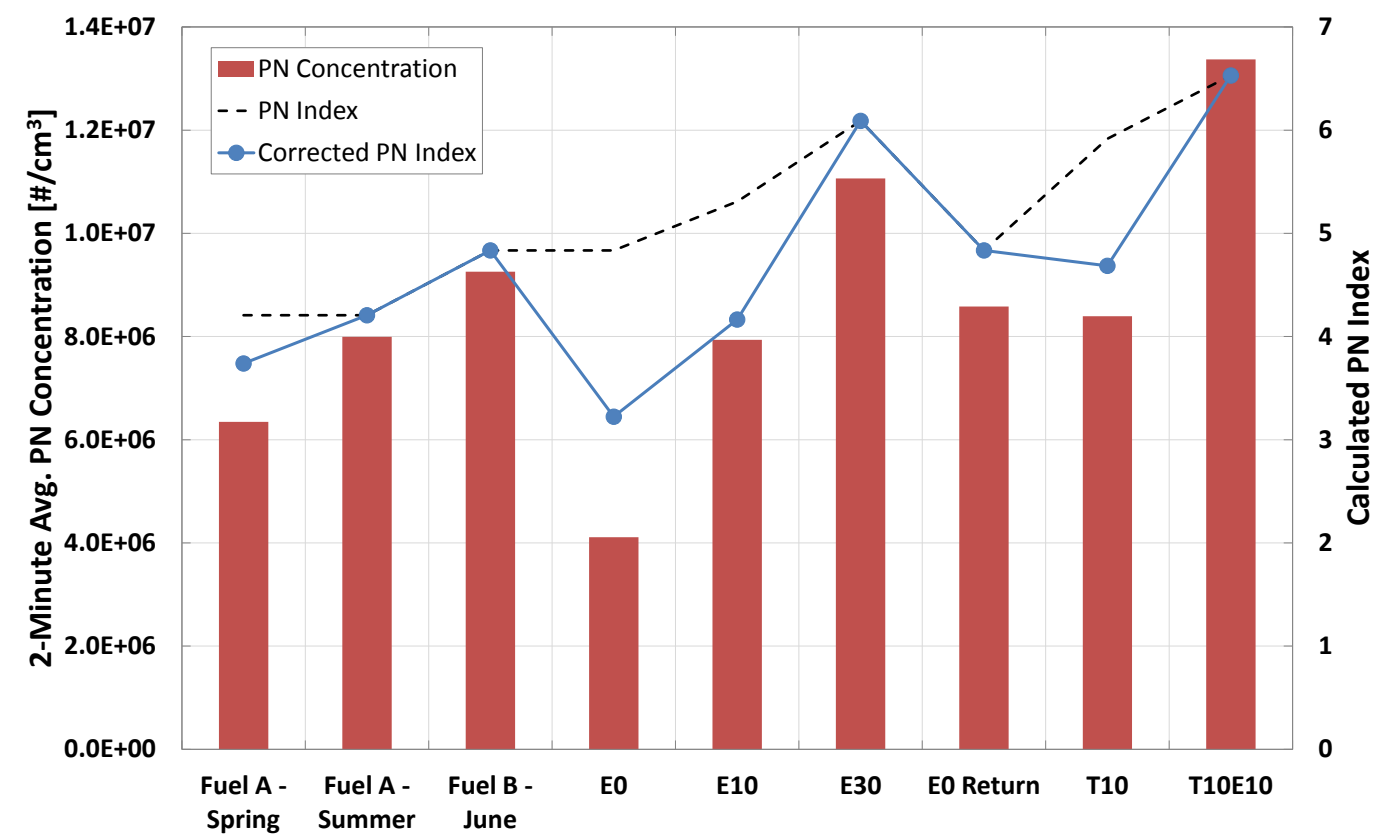

Figure 6.28: PN concentration at run end, averaged for fuel groupings shown, with the corresponding $\mathrm{PN}$ index overlain.

\subsection{Results Synthesis: Explaining the Observed Variability}

This chapter has presented the reader with a great deal of information. This concluding section offers a summary for the key findings presented from a more general perspective.

Through a series of tests, proposed systematic sources of variability from the engine and sampling artifacts were explored. This testing showed that while some changes in PM emissions occurred with the control of various parameters - including dilution conditions, engine temperatures, and overall engine condition - their observed influence was either negligible or lower in magnitude than the observed variabilities. These sources therefore, were found to be inconsequential for explaining observed phenomena of temporal or run-to-run variability.

Inducing changes to the fuel composition through the creation of different fuel blends generated a high degree of emissions variability. Increasing the ethanol or toluene fraction of the fuel caused increases in measured PN concentrations and PM mass, along with seemingly greater emissions variability on a run-to-run basis. Fundamental changes to the particle morphology were observed, evidenced by changes to the particle size distributions and the composition of the PM. Engine behavioural markers that could possibly account for jumps in PM emissions levels were insufficient at explaining the variability seen with different fuel blends. Increasing the ethanol fraction is believed to promote the formation of PM 
due to vaporization issues leading to insufficient fuel-air mixture preparation (i.e. fuel rich cores). On the other hand, toluene provides an increased availability of the PAH pathway for the formation of PM. When these are combined together the results are exacerbated.

Analysing gaseous emissions data more closely identified possible compound markers for fuel compositional changes at the base gasoline level. Indeed, PN concentrations were shown to positively correlate with toluene and ethanol exhaust concentrations; in contrast, a correlation with isobutylene showed a negative response. Analysis of these three compounds shows that they sufficiently describe the measured PM emissions leading to the conclusion that their use as markers is valid. This is corroborated by the fact that changes in measured exhaust concentrations of isobutylene can be used to explain typical changes in gasoline composition for vapour pressure. The use of previously developed PN indices is useful here to provide explanations for fuel changes.

To summarize, emissions presented in this study were not only influenced by the purposeful changes in fuel composition (i.e. the creation of the different fuel blends) but also from unquantified changes to the base fuel composition. These changes at the base gasoline level contributed to changes in $\mathrm{PN}$ magnitude, and more importantly, to run-to-run variability. In spite of this, the emissions data presented here can be used to retroactively explain the observations. 


\section{Chapter 7}

\section{Transient Results}

The results from the transient engine tests are presented in this chapter. Two transient starting procedures were performed - a cold and hot start. Experimental procedures for both starts are contained in Section 5.1.2. The test matrix presented in Figure 6.1 shows the breakdown of transient tests performed. Note that only data collected during the fuel composition portion of the investigation (from test group E0 onward) is presented here for discussion. Therefore, the discussion here centres around the influence of fuel composition on the PM emissions during these two start procedures.

As before, PM emissions data (number concentration, size distributions, and composition) have been corrected for the true, measured DR. Likewise, $\mathrm{O}_{2}$ measurements have been corrected for compound interference. Gravimetric measurements were not performed for the transient test procedures due to difficulty collecting sufficient PM on a filter for statistical relevance in the short period - a result also seen in previous work on this engine [44]. Supplementary start data is included in Appendix D.

\subsection{PN Concentrations and Size Distributions}

The PN concentrations and size distributions from the cold and hot starts are presented in this section. Results are grouped by the tested fuel blend and represent the average of several runs, unless otherwise noted.

\subsubsection{Cold Start}

The test group averaged PN concentrations with run-time for a cold starting procedure are presented in Figure 7.1. In general, the PM emissions follow the behaviour of the engine during the starting procedure; refer to the left-most portion of Figure 5.1 for typical engine speed behaviour during a cold start. A large initial spike of particles occurs when the engine starts after the initial cranking period (a couple of seconds) has elapsed - the characteristic rev up in speed during starting. The PN concentrations then begin to fall while the PCM continues to control the engine until it enters closed-loop fuel control (at approximately 20 seconds) when the oxygen sensor is sufficiently warm to provide accurate readings. This initial starting period culminates with a secondary spike of particles around the 30 second mark, which corresponds with the point where the engine's idle speed is stabilized. AFR data shows that this spike in particles is also aligned with the oxygen sensor integrity check - where the PCM purposely enriches the fuel charge to see if the sensor responds. Beyond this point, the PM emissions continue to 
decay at a more or less steady rate as the engine continues to warm-up with no further speed change. It is worth noting that a cold start is a highly variable endeavour and anecdotally speaking, no two cold starts behave the exact same way. However, the general behaviour described above is applicable to all tests.

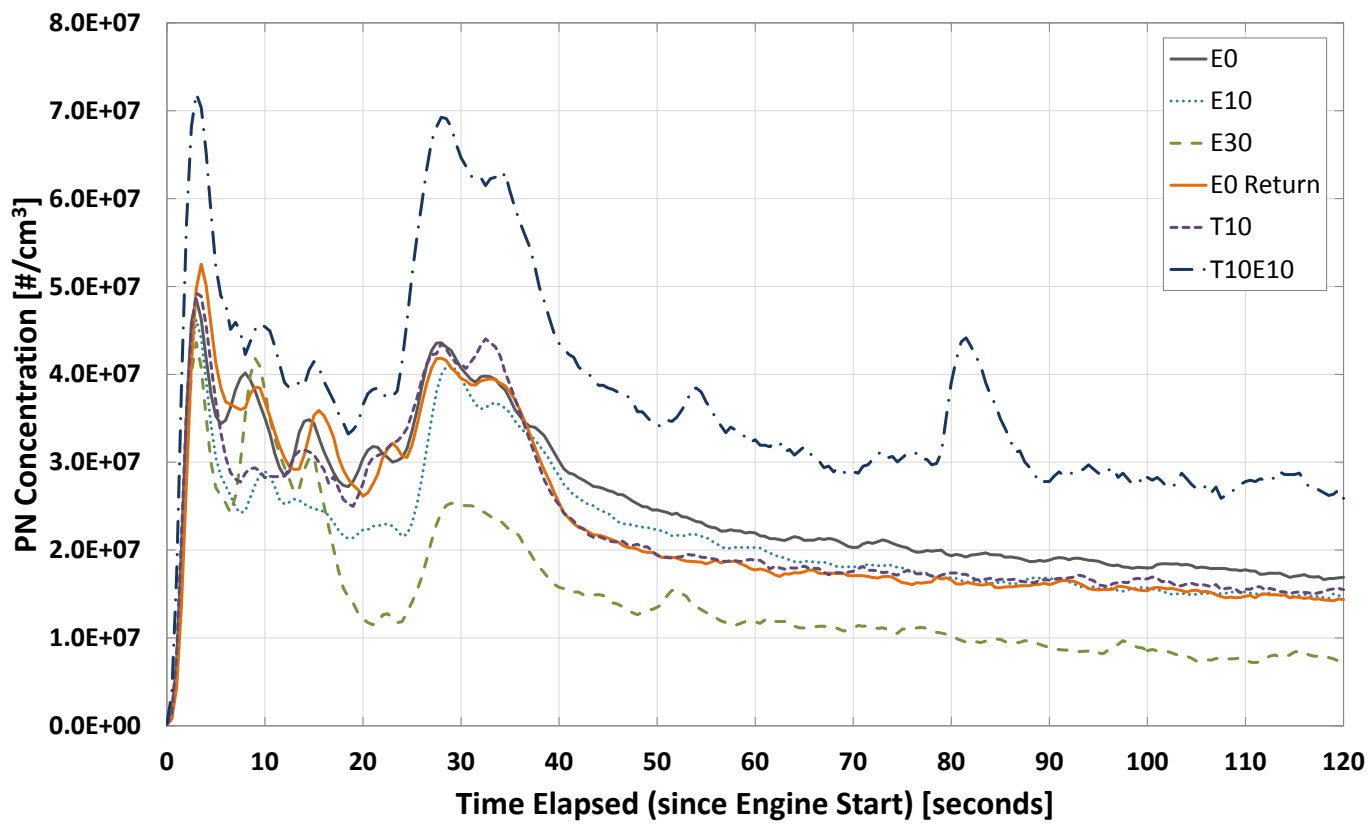

Figure 7.1: Fuel blend comparison of average cold start PN concentration with time.

Comparing the different fuel blends in Figure 7.1 shows some interesting trends. Of the ethanol fuel blends, only the E30 data set showed discernible, positive reductions in PN concentrations. Both E10 and T10 exhibited very similar behaviours to the pure gasoline fuel (E0 and E0 Return). In contrast, the T10E10 fuel blend appears to have resulted in substantially more PM emissions, both in terms of the magnitudes of the PN concentration spikes in the front half, and the steady decay during the latter warm-up period. It should be noted that only one cold start test was performed with the T10E10 blend due to time constraints. Comparing runs on an individual basis shows that the very high initial peak is likely anomalous to some extent. However, no other run with any other fuel blend showed as high of a second peak as with the T10E10 fuel, including the stable idle period afterwards; the tertiary spike of particles seen at around 80 seconds is atypical for the starting procedure. One hypothesis is that a large mass of built-up particles within the engine or the exhaust system became dislodged, causing a momentary spike in particle counts; however, this is an unlikely explanation because particles in such a built-up mass would probably be much larger in diameter than this particle sizer's size range (5.6-560 $\mathrm{nm})$. The increased emissions for the T10E10 fuelled test is thought to have occurred for two reasons; first, the latent heat of vaporization of ethanol causing evaporation issues for the fuel charge, and second, the presence of increased aromatics in the fuel which show a high propensity for soot production due to their ability to form PAHs [21]. It is the combination of both of these factors that contributes to the large emission increase seen here. The issue of ethanol vaporization is thought to not be seen in the E10 blend because it is counter balanced by the ability of the oxygenate fuel to help oxidation during combustion. Similarly, the high aromatic fraction in the T10 blend is not sufficient to cause a strong 
response in PN concentrations because evaporation issues are not present. However, when combined together a response is elicited; a similar finding to the steady-state tests presented in Chapter 6 .

Returning to E30, an explanation for its reduced particle output is offered here. The magnitudes and behaviours of the initial peaks were very similar to the lower oxygenate fuels, but a deviation was observed once closed-loop fuel control was entered at about the 20 second mark. Recalling that the secondary spike is attributed to fuel charge enrichment for oxygen sensor diagnostic purposes, the reason why the E30 fuel produces less PM is likely due to the abundance of oxygen atoms present during combustion. These extra oxygen atoms help to oxidize the regions of rich fuel mixture present in the fuel-air charge in the cylinder, which are exacerbated by poor mixture preparation during cold and slow operation, like that of a cold start. This is also useful in explaining why no observable difference is found between the lower oxygenate (E10) or aromatic fraction blends (T10); the mixture preparation is so poor that it makes very little difference in terms of fuel chemistry.

Figure 7.2 provides the instantaneous size distribution plot with time, averaged for the E0 test group; a plot of average total PN concentration is overlaid above for reference. Generally speaking, the beginning of the starting procedure (0-5 seconds) - during and immediately after the engine cranking - is characterized by accumulation mode particles in the 70-150 $\mathrm{nm}$ size range. The following period, where the engine PCM attempts to achieve stable idle and closed-loop fuel control, shows a transition down to nucleation mode sizes, with particles mostly in the 20-100 nm range. After the oxygen sensor integrity checks are performed, very fine particles with a modal diameter of around $10 \mathrm{~nm}$ are emitted for the remainder of the test. The other test fuels (see Appendix D.1 for similar plots for the other test fuels) displayed a similar particle size behaviour with some differences and size shifts. The results here agree with those found in other studies at similar operating conditions, namely those by Samuel et al. [25] and Dimou et al. [24]. Both of these studies report that nuclei particles dominate under cold fast idle conditions, even with a wide range of ethanol blends (E0-E85).
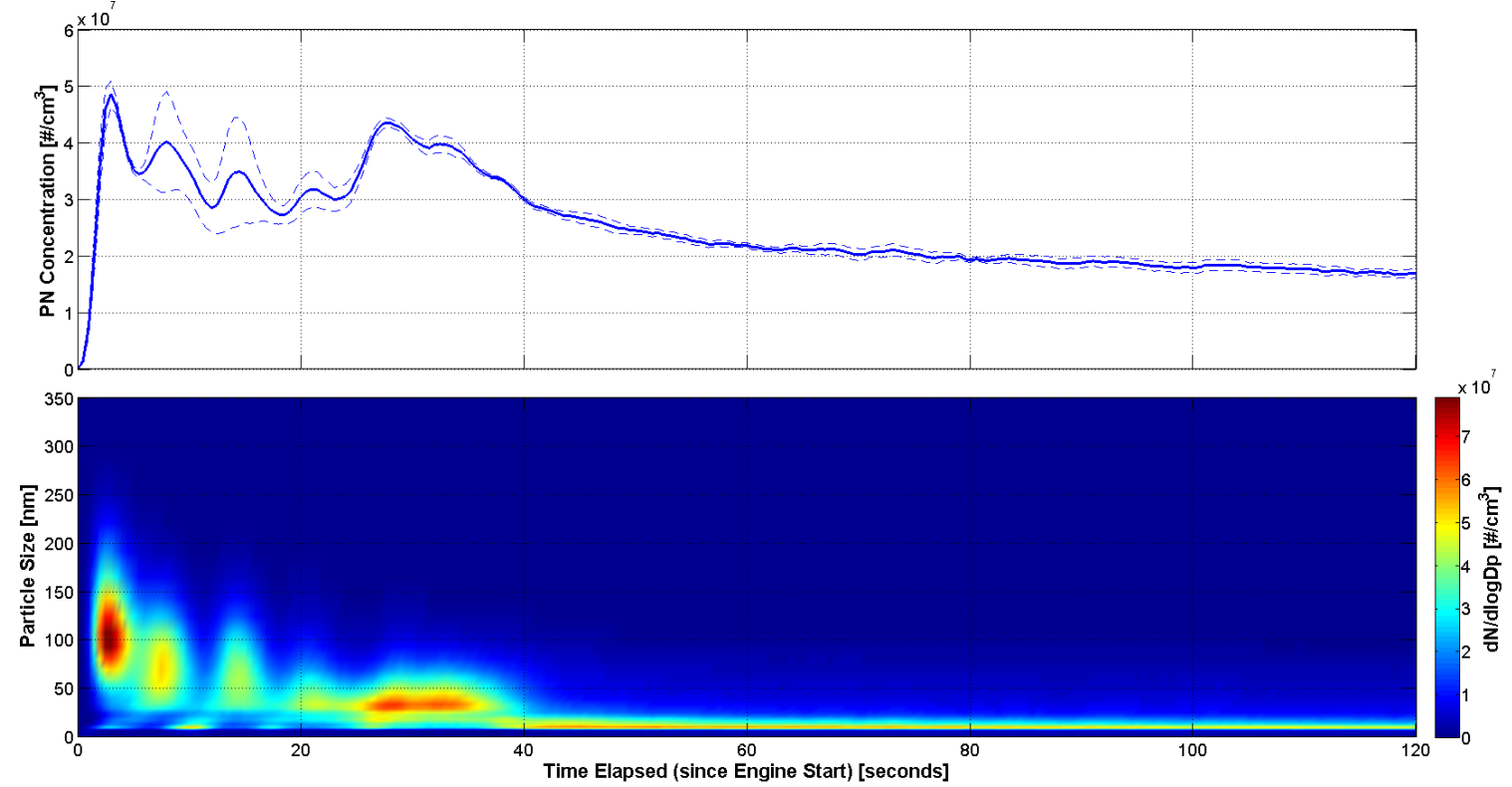

Figure 7.2: Average cold start PN concentration (top) and particle size distribution (bottom) with time for E0 fuel. Dashed lines in top figure indicate standard error. 
Figure 7.3 presents cumulative size distributions during cold starts for each of the test groups investigated. Presenting the data in this way allows for analysis into the relative changes in particle size distributions between the different fuel blends over an entire cold start period. Here it is evident that nucleation mode particles dominate over the entire start period, even though "bursts" of larger particles occur very early on. This observation holds true regardless of the fuel blend used, which again agrees with relevant literature [24, 25]. The general distribution shape is guided by the three start phases discussed above, with the initial starting period accounting for particles $>25 \mathrm{~nm}$. In terms of the distribution shape, only the E30 test group deviated strongly from the general shape observed. Inspection of the time resolved distribution data for this test group shows that the initial accumulation mode peaks shifted to smaller sizes when compared to the other fuels. Furthermore, the secondary spike caused by the oxygen sensor check, which gives a second "bump" of particles in the 25-50 nm range, was diminished for the E30 test group. Therefore, the typical second mode was largely absent when running E30. Using the error bars as a guide, both the E10 and E30 gave statistically significant $(\mathrm{p}<0.05)$ reductions in particles greater than $30 \mathrm{~nm}$ and $15 \mathrm{~nm}$, respectively. Comparing the fuels at the nucleation mode (10 $\mathrm{nm}$ ) size range shows there were increased emissions for E10 over E0 over the duration of a test, while the E30 test group provided reductions at this size. It is worth noting that previous work on this engine showed similar cumulative distributions, including the observed ethanol responses [44]. Analysing the toluene blended fuels shows that a possible shift towards larger particles occurred, with a corresponding decrease in peak cumulative nucleation mode particles.

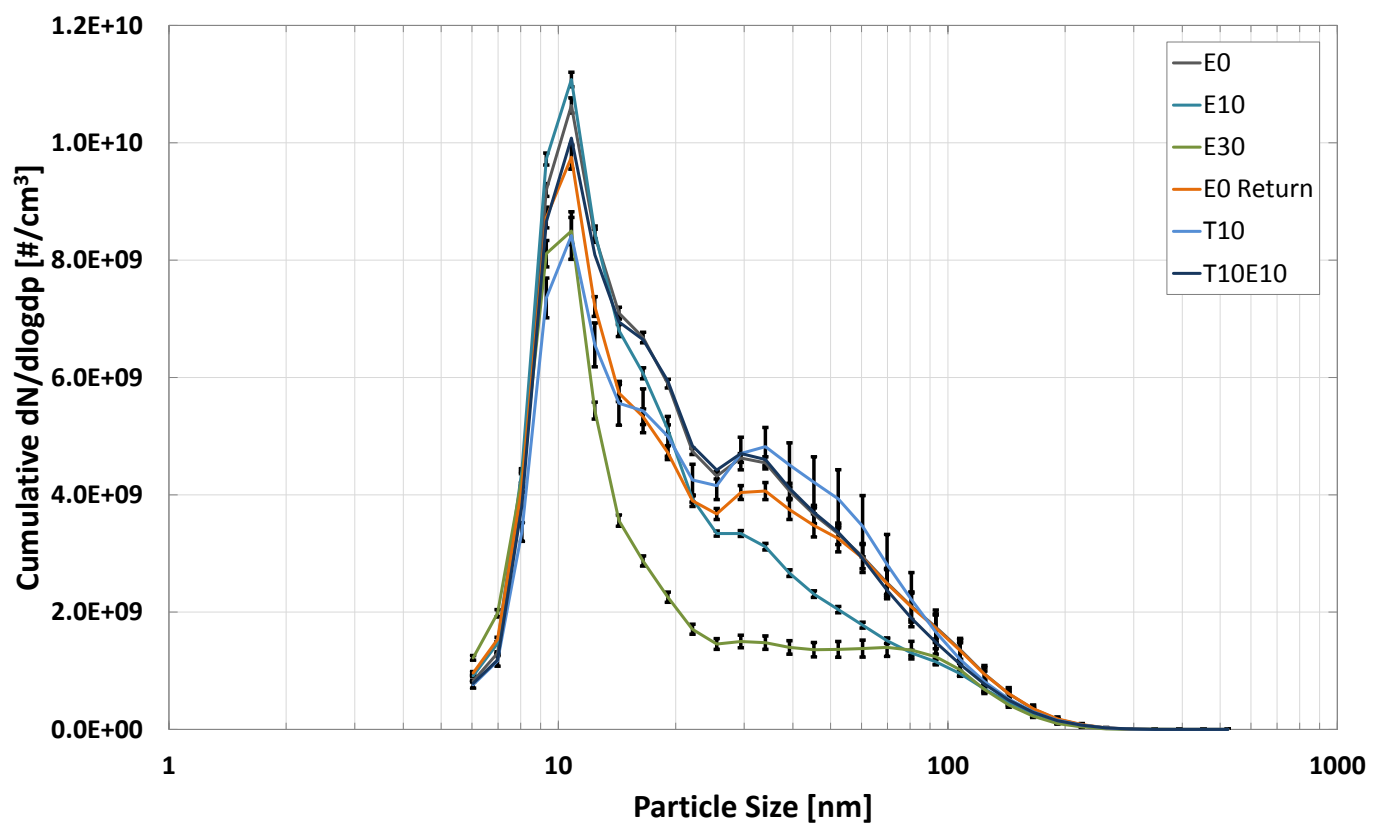

Figure 7.3: Cumulative normalized concentrations (dN/dlogdp), averaged for each test fuel under cold start testing. Error bars indicate $95 \%$ confidence interval from each group - error bars not shown for T10E10 (one sample). 


\subsubsection{Hot Start}

The PN concentration results for the hot start tests are contained in Figure 7.4. Referring back to Figure 5.1 provides a basis for engine behaviour during a typical hot start procedure. Just as in the case of the cold starts, the PM emissions during a hot start closely follow the engine speed behaviour, with an initial spike of particles as the engine starts. The major difference here however, is that idle and PM emission stabilization occurs very soon after the engine is started. This is directly attributable to the fact that the engine and, more importantly, the oxygen sensor are both at fully heated operating temperatures. This means that soon after the engine is started it enters closed-loop fuel control and stable idle without the need for a oxygen sensor integrity check. As a result, the secondary peak of particle emission typically seen in a cold start test is not present during hot start. Moving on to the stable idle period shows that a steady PM emission decay is not observed like it is for the cold start procedure. This is due to the fact the ECT does not increase during this period like it does in the cold start test. It should be noted that the data presented for the hot start represents single runs; multiple runs were not performed due to time constraints. However, hot starting is typically very repeatable in terms of PM emissions and engine behaviour; a conclusion also found in previous work on this engine [44]. Therefore, the presented data is still considered useful for analysis, though without statistical commentary.

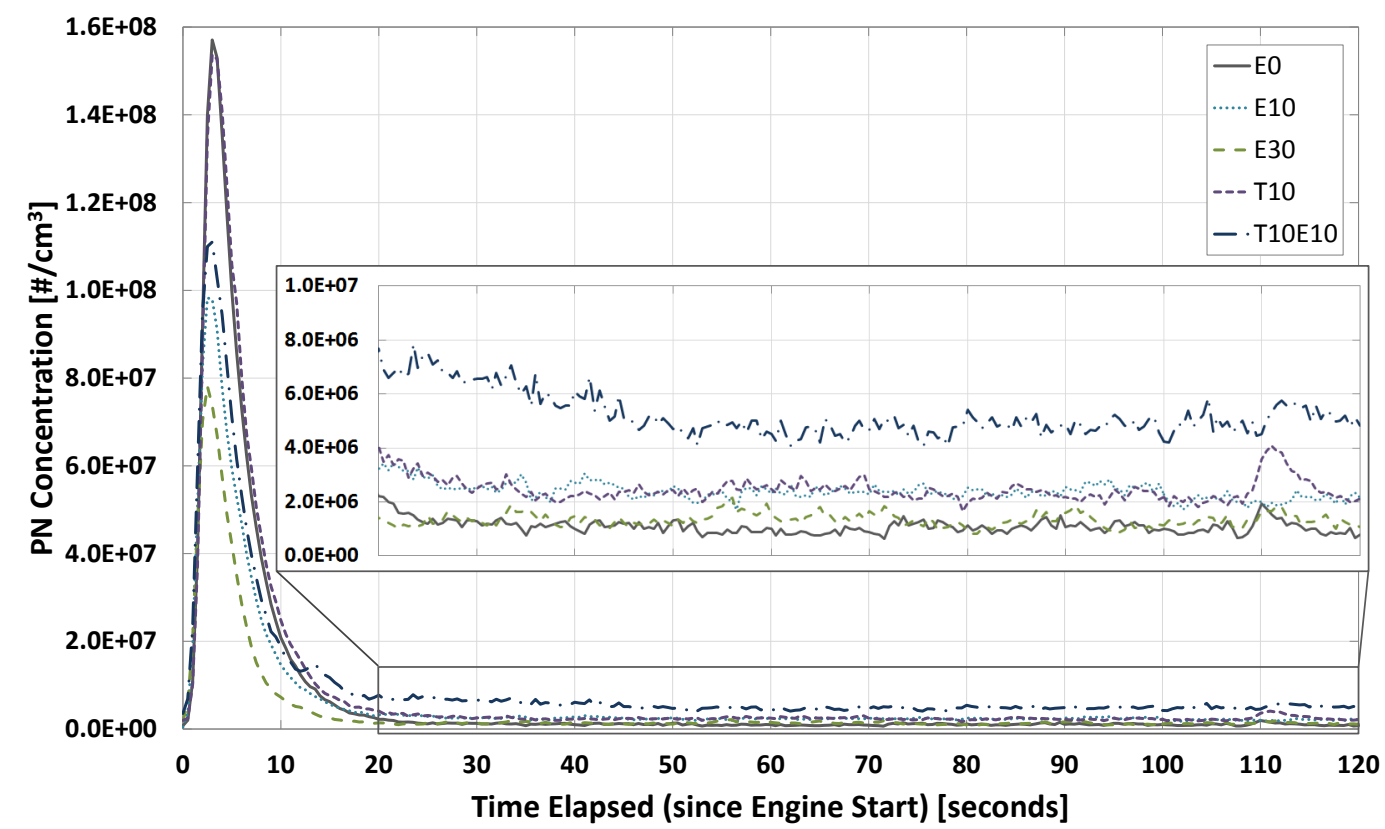

Figure 7.4: Fuel blend comparison of hot start PN concentration with time.

The comparison of fuel composition shows a more pronounced effect of ethanol in hot starts than it does in the cold starts. Analyzing the magnitudes of the initial spike of particles in Figure 7.4 shows reductions even for low ethanol content fuels. Of note, E0 and T10 tests show no discernible difference in PM emission behaviour. Likewise, the T10E10 and E10 blends provided very similar but reduced peak PN concentration magnitudes, while the highest ethanol content blend (E30) yielded the greatest reductions in peak PM emissions. These results indicate that ethanol played a seemingly more important role than the aromatic (toluene) fraction of the fuel during the initial spike of particles. Conversely, differences were observed when going beyond the initial peak; the above mentioned ethanol 
benefit was somewhat reversed during the steady idle period. The overlain chart in this figure provides a more appropriate scale for this period - note that the time axis lines up accordingly here. The E0 and E30 fuel blends emitted the fewest particles during this period, while the E10 and T10 fuel blends emitted at an elevated rate - roughly double. The T10E10 blend, which did not emit the highest PN concentrations during the initial peak, appears to have emitted the most particles during the steady idle portion of the hot start; roughly double the concentration of either the T10 or the E10 blends. This again demonstrates the cumulative effect of having both ethanol and toluene blended into the fuel just as for the cold start test. The same explanation is applicable here: the high latent heat of vaporization of the ethanol causes inhomogeneities in the fuel charge which leads to favourable conditions for soot production, augmented by the presence of excess aromatic compounds (toluene) with a high degree of sooting propensity [21].

The particle size distribution during the hot start period, averaged for the E0 fuelled tests, is given in Figure 7.5; a plot of PN concentration with time is provided above. This figure represents the typical hot-start distribution regardless of the fuel used, though similar plots for the remaining fuel blends are provided in Appendix D.3. Similar to the cold start tests, inspection of this plot shows the initial peak of particle emission to be dominated by particles in the 100-200 $\mathrm{nm}$ size range. Immediately after this peak the measured PN concentrations decay almost an order of magnitude, and the distribution shifts towards smaller particles.

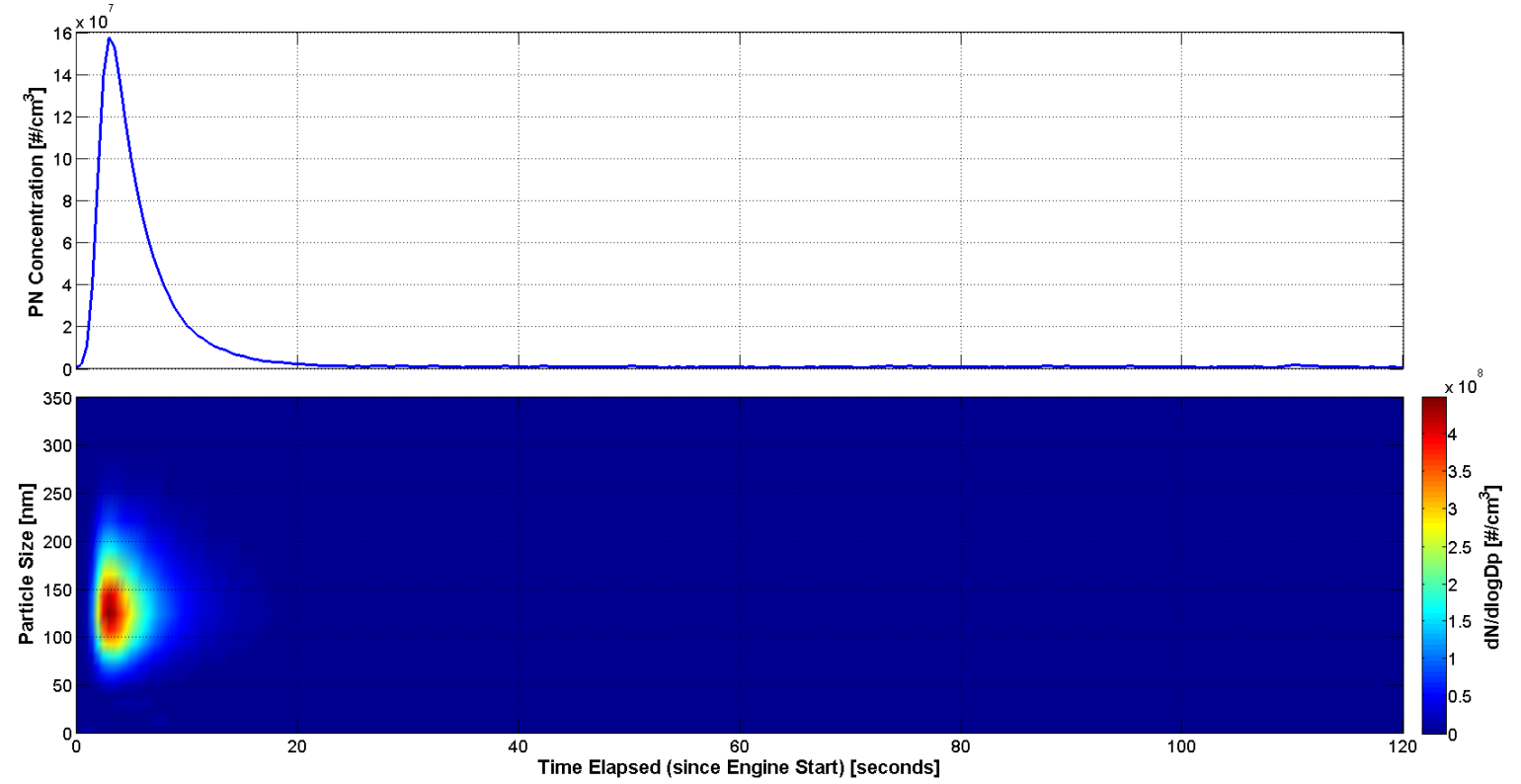

Figure 7.5: Hot start PN concentration (top) and particle size distribution (bottom) with time for E0 fuel.

Cumulatively speaking, this initial peak accounts for the majority of the particles emitted during the two minutes of the test. As a result, the distributions shown in Figure 7.6 are dominated by these initial accumulation mode particles, regardless of fuel used. There was, however, a pronounced fuel effect on the shapes and magnitudes of the cumulative distributions. Generally, increasing the ethanol content lowered the modal diameter and reduced particle counts in the accumulation mode size range. An opposite effect was seen in the smaller size bins, where ethanol caused an increase in emission. Both 
of these observations were seen in previous work on this engine [44]. Interestingly, the higher aromatic fraction fuels (T10 and T10E10) show small shifts to larger particles when compared to their respective ethanol counterpart; i.e. T10 vs. E0 and T10E10 vs. E10. As was observed with the cold start tests, a combined effect of ethanol and toluene seems to exacerbate the emission of PM from this engine during the 20-120 second time frame. Increasing the ethanol content further, to $30 \%$ by volume, gave the greatest reductions in the accumulation mode, and matched the emissions of $\mathrm{E} 0$ at the smaller size bins. Therefore, the addition of ethanol can be said to positively reduce peak particle emissions during a hot start test. These peak emissions account for the majority of the emitted PM during this period.

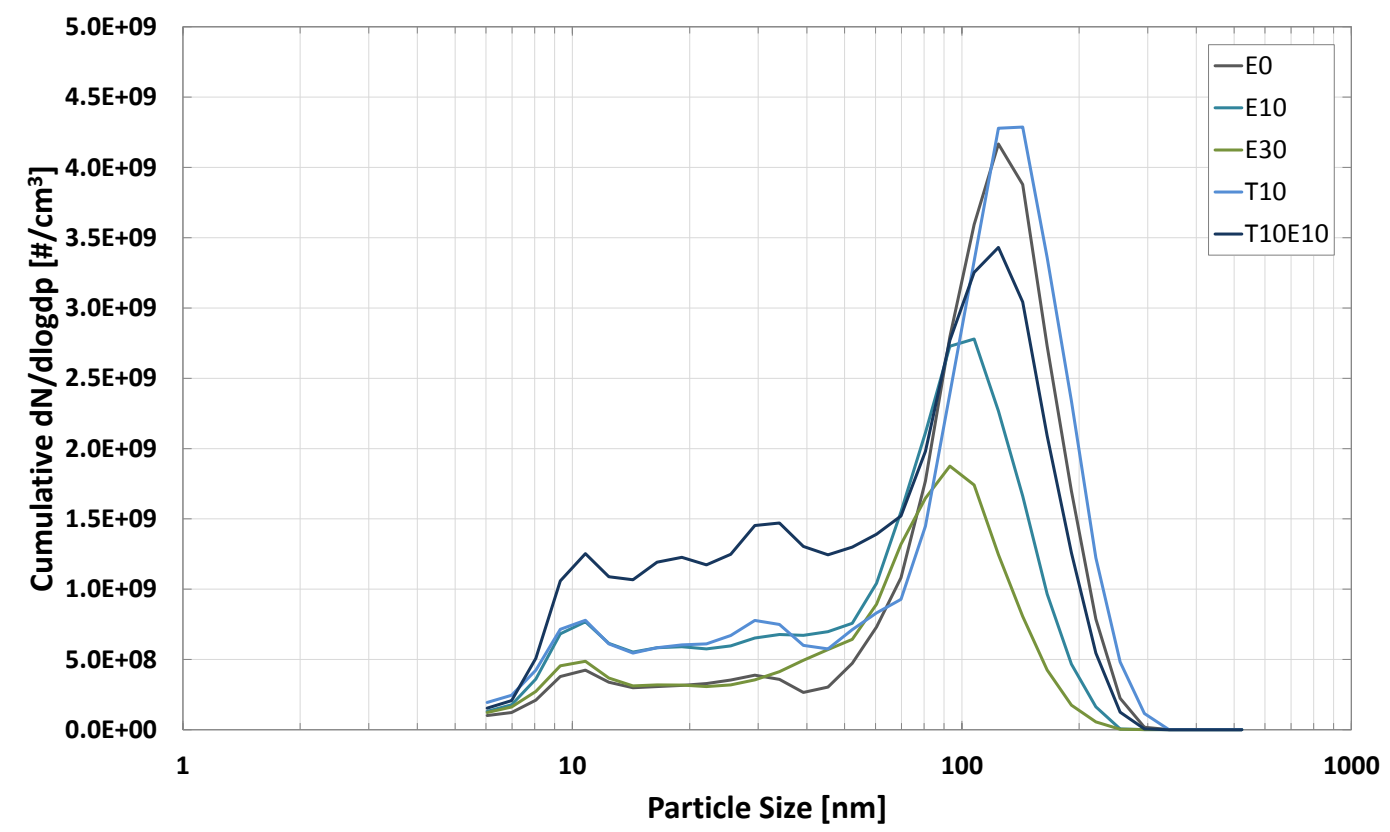

Figure 7.6: Cumulative normalized concentrations (dN/dlogdp) for each test fuel under hot start testing. Only a single run (sample) for each test fuel was collected.

\subsubsection{PN Totals}

Summing the PN emissions over the entire start test length synthesizes the small scale effects described in the previous discussions and provides for a macro level comparison of emissions over the entire test. PN concentration totals for both cold and hot starts broken down by fuel blend are presented in Figure 7.7. Cold start data is averaged for multiple tests of each fuel blend, except for T10E10; hot start data is from singular runs for each fuel blend. The influence of fuel composition can be distinguished by using the two gasoline test groups (E0 and E0 Return) as baselines. Using E10 showed modest reductions of $14 \%$ on average over E0, while using E30 lowered measured PN concentrations by $42 \%$ over a cold start test. It should be noted that on average, the E0 Return test group emitted fewer particles than the E0 group, even though the same fuel blend was used. This is another example of how the variability of the fuel composition at the commercial level can make fuel based comparisons rather difficult, as was the case with ethanol in this investigation. No statistically significant ( $>00.05)$ change in measured PN concentration was noted for the T10 fuel blend compared to the E0 Return data, though lower total concentrations were seen compared to the original E0 data set. In contrast, the T10E10 blend emitted 
substantially more particles than pure gasoline (E0 or E0 Return). This result combines well with the plotted PN concentrations with run time in Figure 7.1, where no appreciable difference was seen for either the E10 or T10 fuel blends when compared to either the E0 or E0 Return data, while the T10E10 blend did show differences. This was attributed to the combined effect of both the ethanol and toluene in fuel increasing the measured PM emissions. Another important take-away from Figure 7.7 is the magnitudes of the $95 \%$ confidence intervals for the other test groups. While only one T10E10 test was performed in this investigation, since the other test groups show relatively small confidence bounds this large increase in cumulative PM is likely not a statistical anomaly. Of course, the collection of further data would provide some statistical confidence and doing so in future work would be beneficial. Comparing the hot start data shows a similar trend to the cold start behaviour, where a decrease in PM emissions tracked with increasing ethanol content. Increasing the toluene content of the fuel blend increased the measured total PN concentrations, though the combined effect of ethanol and toluene with the T10E10 is not as strong as was observed for the cold start test. Since only one test was performed for each fuel blend, it is impossible to comment on the statistical significance of these trends, though it should be reiterated that hot start tests are highly repeatable both in terms of engine and PM emission behaviour.

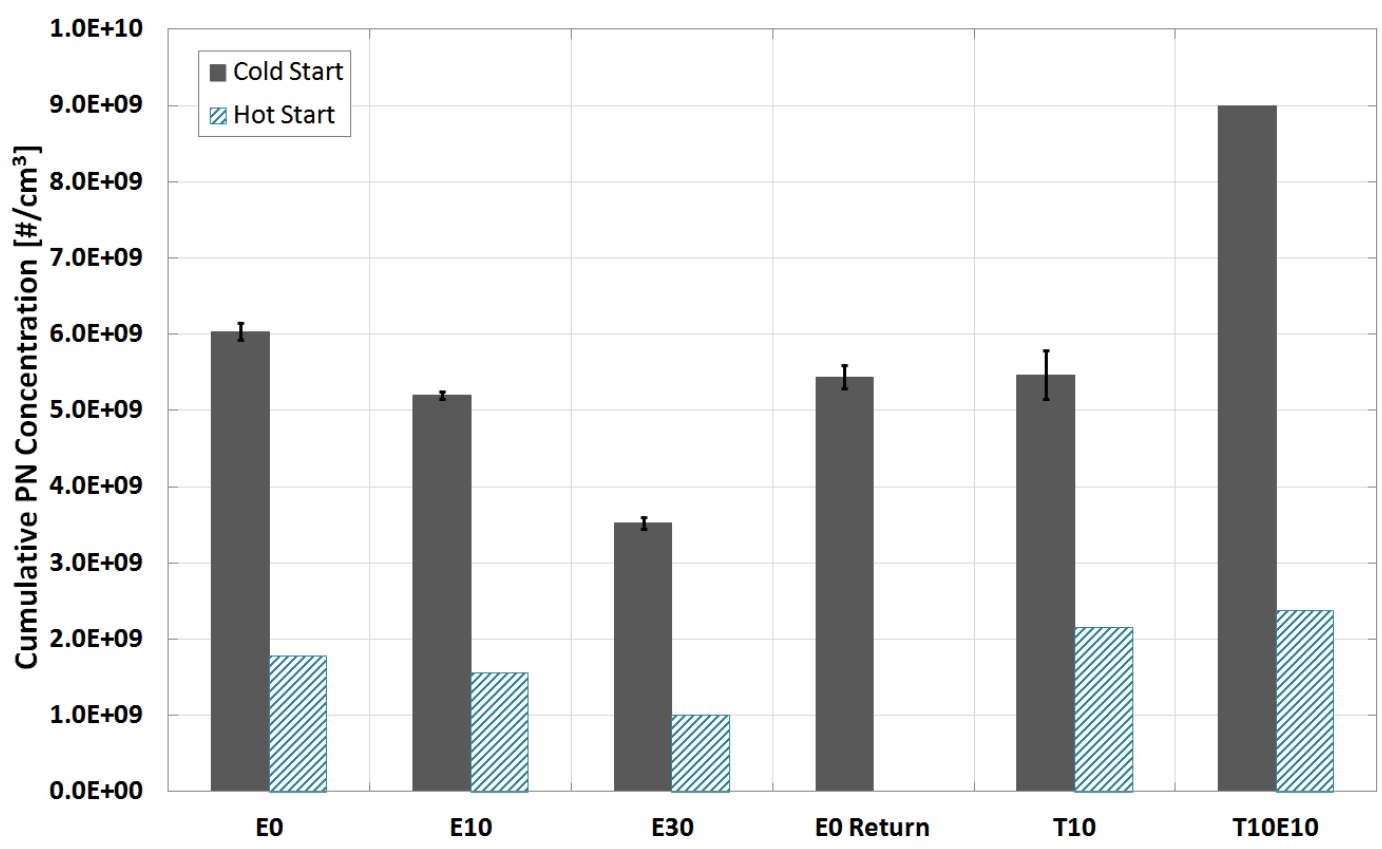

Figure 7.7: Fuel blend comparison of total PN concentration during cold and hot starts. Error bars indicate $95 \%$ confidence interval - data without error bars are from single runs.

\subsection{PM Composition}

PM compositional analysis (EC/OC) was performed on PM laden filters collected during the engine start tests. Test group averaged concentrations and EC/OC ratios are summarized in Figures 7.8 and 7.9 for the cold and hot start tests, respectively. No filters were collected for the E0 Return and T10E10 test groups due to equipment scheduling conflicts. A note for the reader; the error bars here represent the $95 \%$ confidence interval from multiple punches for one test of each fuel, not multiple tests. Before discussing 
the results, it is worthwhile to note that punch handling was sometimes problematic during this analysis. As an example, only one punch was deemed correctly handled for the T10 hot start results. Therefore, these error bounds do not necessarily imply experimental errors, but rather measurement errors from a single experiment.

Considering the results from both starting procedures, no test fuel resulted in an emission of more elemental carbon than organic carbon; i.e. $\mathrm{EC} / \mathrm{OC}<1$. Historically, this has been typical for PFI engine particle composition, where condensed volatiles form the bulk of emitted PM. However, Maricq et al. [92] found the opposite for a GDI engine under starting conditions. This disagreement can be explained by considering that the TWC equipped in their study removed the organic portion, leaving the PM as primarily elemental based - no TWC is equipped on this research engine. The high organic portion emitted by this research engine also highlights the need to perform this analysis as soon as possible after collection in order to mitigate the evaporation of these species. Given the $95 \%$ confidence intervals presented, this result is statistically significant for all test fuels; i.e. all test fuels' error bounds do not cross the threshold of ratio unity. In general, cold starts resulted in a higher fraction of organic carbon compared to the hot start tests, though this cannot be said with confidence because of the interval overlaps shown. This is the expected result because the cold start is mired by fuel evaporation issues from a cold engine, providing an abundance of UBHCs to condense and coat existing particles or to form nuclei particles themselves. This is consistent with the aforementioned study [92], where higher concentrations of organic carbon were noted for the cold start phase.

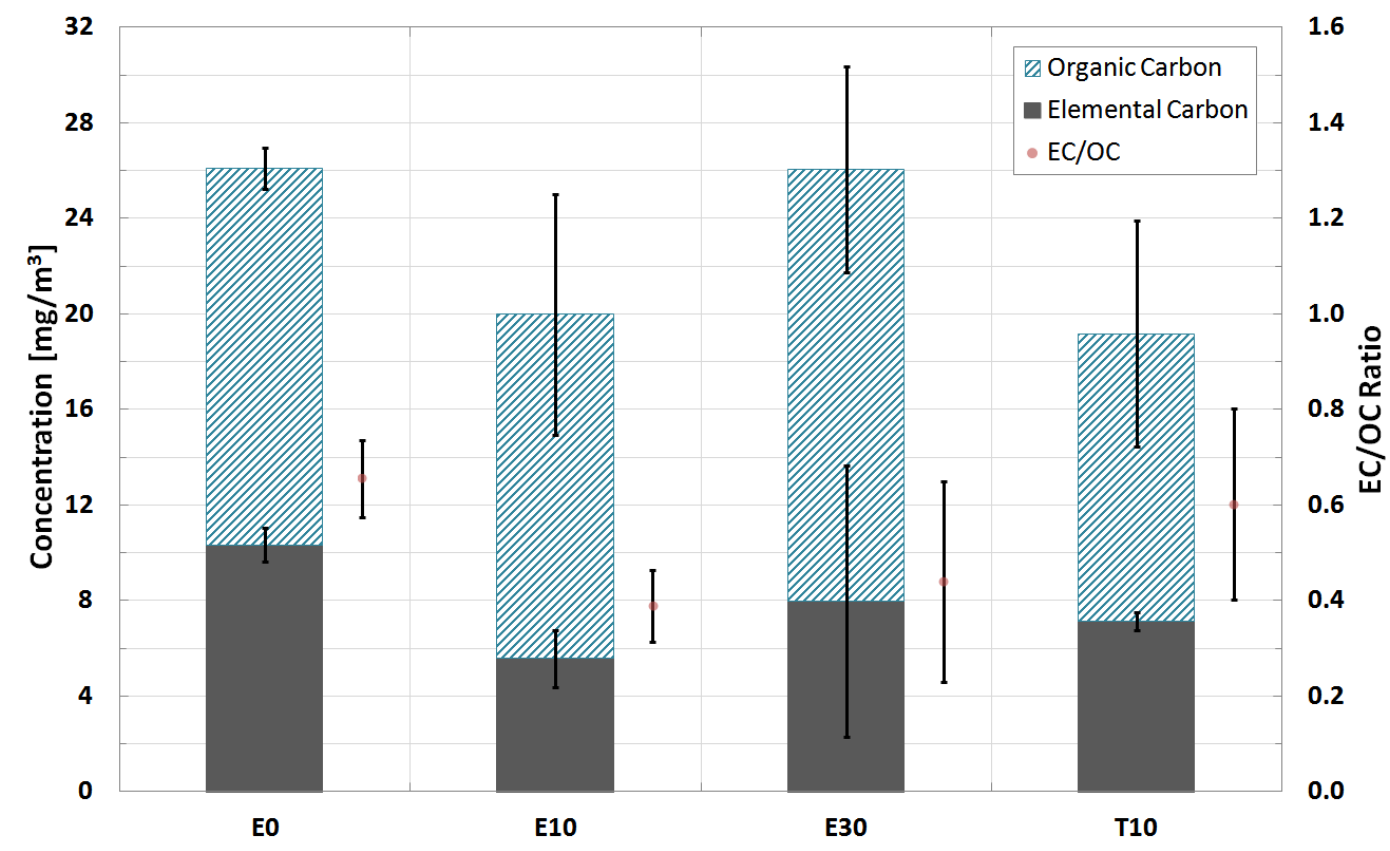

Figure 7.8: Cold start elemental and organic carbon concentrations. Error bars indicate $95 \%$ confidence intervals.

In terms of fuel composition, statistically significant $(\mathrm{p}<0.05)$ changes in the EC/OC ratio were seen when comparing the E10 and E0 data under either starting situation. For the hot start test, the E30 test fuel appears to have dropped this ratio further, though this is right on the edge of statistical relevance. Maricq et al. [92] also showed a similar trend with increasing ethanol content providing for a greater proportion of organic carbon. Considering the concentrations shown in these two figures 
provides some insight to how the proportions change. Under the cold start test, the observed reduction in $\mathrm{EC} / \mathrm{OC}$ ratio from E0 to E10 by a factor of 1.7 was due to a disproportionate reduction in the elemental concentration - both elemental and organic concentrations decreased in this case. For the hot start test, the same two fuels showed an average of the same total (elemental + organic) concentration, so the difference here was due to a proportionate change; i.e. the reduction in the elemental concentration is offset by the increase in the organic concentration. Moving on to E30 from E10 shows that the elemental concentrations remained constant while the organic concentrations increased, thereby, dropping the calculated EC/OC ratio further for the hot start test; the same cannot be said for the cold start test with statistical certainty. The implication of these results is that total carbon measurements (elemental + organic) can vary in a different manner than the EC/OC ratio. Therefore, it is necessary to consider emissions results harmoniously to contextualize the results as a whole.

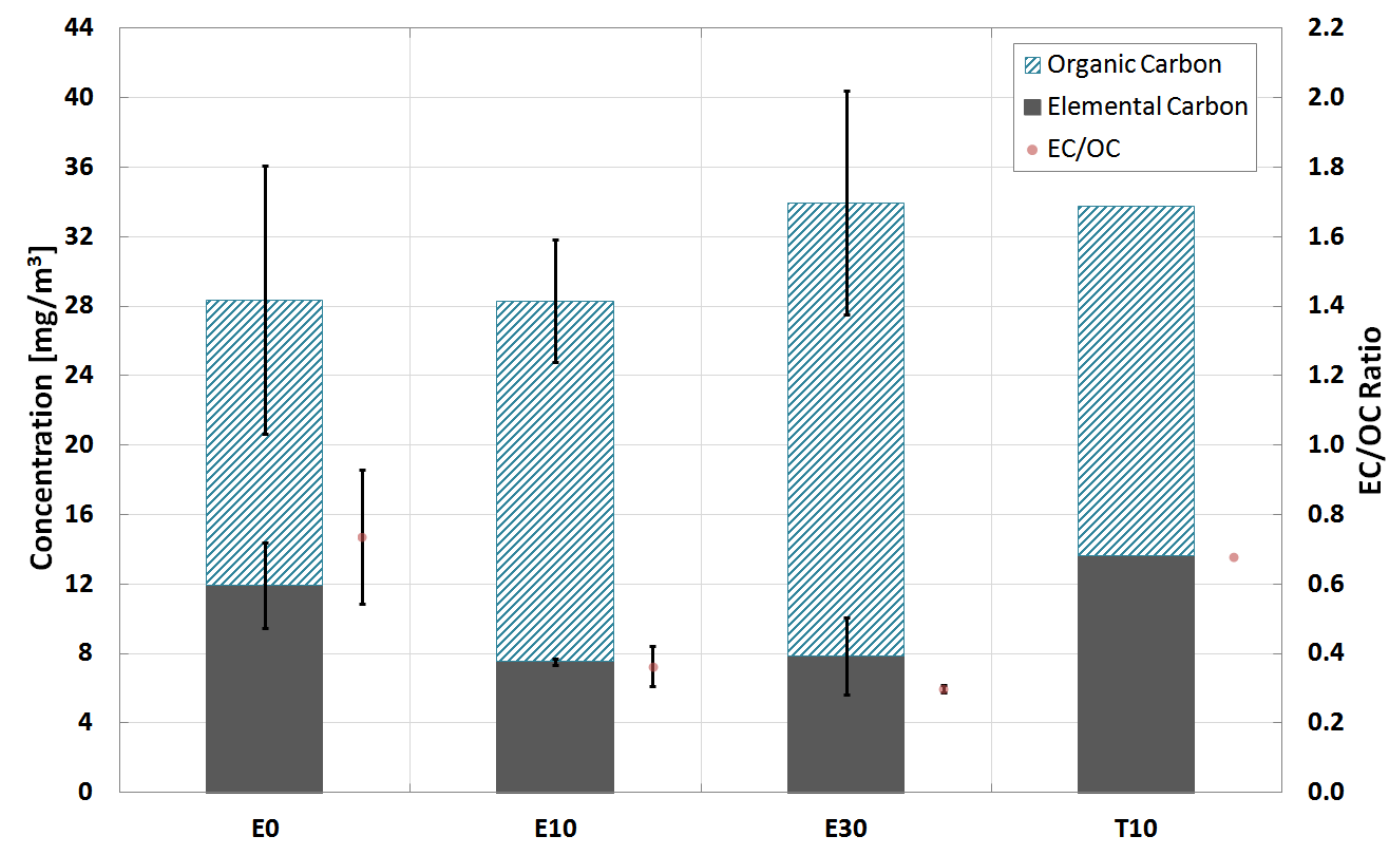

Figure 7.9: Hot start elemental and organic carbon concentrations. Error bars indicate $95 \%$ confidence intervals - error bars not shown for T10 (one sample)

\subsection{Gaseous Emissions}

Turning to the gaseous emissions may provide some insight into the discussed PM trends. Gaseous emissions from the standard emissions bench are reported here for both engine start tests. The standard emissions bench reports $\mathrm{THC}, \mathrm{NO}_{\mathrm{x}}, \mathrm{CO}, \mathrm{CO}_{2}$, and $\mathrm{O}_{2}$ concentrations. The $\mathrm{O}_{2}$ concentrations have been corrected for compound interference (primarily $\mathrm{NO}_{x}$ ).

\subsubsection{Cold Start}

The cold start gaseous emissions figures are contained in Appendix D.2. A summary of the gaseous emissions during the cold start is provided below. 


\section{THC}

Much like the PM emissions noted in an earlier section of this chapter, the THC emissions - reported here as $\mathrm{C}_{3}$ - follow the behaviour of the engine during the cold start. A large spike of UBHC emissions occurs during the initial cranking period, followed by a drop while the PCM attempts to achieve stable idle. The emissions subsequently climb up until the engine enters closed-loop fuel control and the diagnostics test of the oxygen sensor is carried out. The emissions then stabilize with the stable idle period and decay at a monotonic rate as the engine warms up. When comparing the test fuels, no differences were seen during the initial starting period (0-40 seconds), except for E30. The engine experienced some difficulty in cold starting with the E30 fuel, so additional spikes of UBHC emissions were seen due to fuel vaporization issues from the high latent heat of vaporization of ethanol. Further differences in THC concentration were seen once stable idle was achieved (after 40 seconds). During this stable idle period, the amount of ethanol in the fuel appears to have a positive effect on reducing THC concentrations. E30 provided the greatest reductions, while E10 and T10E10 showed decreases when compared to their non-ethanol containing counterparts; E0 and T10, respectively. This result is easily explained by the fact that oxygenate fuels, by their very name, carry with them oxygen that become available for oxidation when their molecules are decomposed during combustion. This available oxidizer is then consumed by the burning fuel, resulting in lower UBHC emissions.

\section{$\mathrm{NO}_{\mathbf{x}}$}

NO production is highly temperature dependent and relies on $\mathrm{O}_{2}$ and atmospheric $\mathrm{N}_{2}$ abundance [8]. Therefore, in IC engines two requirements for $\mathrm{NO}_{\mathrm{x}}$ production are required: high cylinder and exhaust temperatures, and the availability of oxygen. Both of these requirements are satisfied during the initial engine start here. During a start, the commanded throttle position is set moderately high (19.1\%) to help begin starting; a throttle position of $15.7 \%$ is normally used by the PCM for cold idle. This causes in-cylinder peak temperature to be higher than it would during idle at a time where fuel utilization is low (excess oxygen) - see THC concentration spike in discussion above. These two factors combine to form the large spike in $\mathrm{NO}_{\mathrm{x}}$ concentrations measured at the beginning of the starting period. These concentrations drop almost immediately once the idle speed and AFR stabilize. Comparing the different fuel blends shows a decrease in $\mathrm{NO}_{\mathrm{x}}$ emissions with increasing ethanol content. Throughout the cold start period E30 $\mathrm{NO}_{\mathrm{x}}$ emissions were consistently lower than the other test fuels, while the E10 data showed slightly lower emissions during the stable idle period (after 40 seconds). The following reason is provided here; the high latent heat of vaporization of ethanol provides for charge cooling as it evaporates in the cylinder. This charge cooling lowers peak compression temperatures and as a result, lowers peak combustion temperatures as well, thereby mitigating the formation of $\mathrm{NO}_{\mathrm{x}}$ species.

\section{CO}

$\mathrm{CO}$ production is largely controlled by the equivalence ratio [8]; for spark ignited engines, operating at stoichiometric conditions will result in the emission of large concentrations of $\mathrm{CO}$, with emissions climbing steadily with increasing equivalence ratio (enriching). Inspecting the $\mathrm{CO}$ emissions data shows exactly this. The emissions increase to idle levels when the engine is first started. A large secondary peak is observed, which coincides with oxygen sensor integrity check. This is a logical observation because the oxygen sensor check involves enriching the fuel mass, which should give an increase in CO concentrations. 
Following this emission maximum, the concentrations drop down to typical idle levels. In terms of fuel composition, some small decreases in $\mathrm{CO}$ concentrations were noted for an increase in ethanol content. This observation is readily explained by the fact that higher ethanol fuel blends will have lower carbonto-hydrogen atomic ratios than a pure gasoline. The reduction in carbon content results in there being less carbon available to form $\mathrm{CO}$ compounds during rich (or stoichiometric) combustion. There is the additional benefit of available oxygen in the fuel to oxidize the $\mathrm{CO}$ to $\mathrm{CO}_{2}$.

\section{$\mathrm{CO}_{2}$ and $\mathrm{O}_{2}$}

During cold starting, $\mathrm{CO}_{2}$ emissions increase rapidly as the engine is started before reaching typical $13.8 \%$ concentration levels. Likewise, $\mathrm{O}_{2}$ emissions decay rapidly as the engine is started down to typical $1 \%$ concentration levels. No major differences for the different test fuels were seen for either $\mathrm{CO}_{2}$ or $\mathrm{O}_{2}$ emissions.

\subsubsection{Hot Start}

A brief discussion is provided below on the results from each analyzer. The hot start gaseous emissions figures are contained in Appendix D.2.

\section{THC}

THC emissions during the initial starting period of a hot start test were observed to double in magnitude over typical cold start emissions. Because stable idle is achieved soon after the starting begins, emissions drop immediately to their warm idle concentrations, which are slightly lower than typical cold idle conditions because fuel vaporization is enhanced in a fully warm engine. No major differences in emissions were seen for the different fuel blends during this idle period. There appears to be some influence of both ethanol and toluene content on peak magnitudes. The fuel with only toluene added (T10) emitted the most, while adding ethanol (T10E10) appeared to lower this peak magnitude. Likewise, E10 and E30 both showed minor reductions in peak THC concentrations when compared to the E0 fuel. The improvement that ethanol provides is likely due to the fact that it carries with it oxygen that can help oxidize the fuel and result in lower THC emissions.

\section{$\mathrm{NO}_{x}$}

The general $\mathrm{NO}_{\mathrm{x}}$ emission trend follows that of the engine behaviour during the hot start. Immediately following start initiation the $\mathrm{NO}_{\mathrm{x}}$ concentrations rapidly rise to peak emissions, before falling down to typical hot idle levels. An interesting observation is that emissions during stable idle are reduced during the hot start over that of the cold start, for all fuels tested. This may seem counter intuitive, given the thermal dependence of NO formation, but is explained here by the fact that the throttle position during hot idle is less than during cold idle; $14.5 \%$ vs. $15.7 \%$. This throttle angle difference cuts the mass air flow to the engine in half, dropping the intake pressure and more importantly, peak pressure and temperature. Therefore, the reduction seen here can be explained by the lower peak cylinder temperatures under hot idle operation. No ethanol benefit was seen during the initial peak, and only a minor benefit of a few ppm was observed during the stable idle period - greater reductions were observed during the cold start tests. 


\section{$\mathrm{CO}$}

$\mathrm{CO}$ emissions during the hot start are characterized by a large spike during the initial starting, followed by a decay to typical idle emission levels. It is worth noting that peak magnitudes of $\mathrm{CO}$ emission during hot starts were larger than during cold start by up to a factor of three, depending on the fuel. Stable idle emissions (after 20 seconds) were also higher than during the cold starting procedure, but to a much lesser extent. Both of these observations can be explained by the fact that while CO production is largely controlled by equivalence ratio, it also thermally dependent [8]. It should be expected that $\mathrm{CO}$ production is augmented with a fully warm engine. Comparing the test fuels shows that positive reductions in $\mathrm{CO}$ are realized with the addition of ethanol, especially during the initial peak. Once more, the fact that the fuel carries with it oxygen likely helps to drive the oxidation of $\mathrm{CO}$ constituents to $\mathrm{CO}_{2}$. Furthermore, the charge cooling effect permits a reduction of peak cylinder temperature during combustion. These peak reductions appear much stronger for the hot start than for the cold start. Finally, the displacement effect the ethanol has on the carbon content results in fewer available carbon atoms for the formation of $\mathrm{CO}$.

\section{$\mathrm{CO}_{2}$ and $\mathrm{O}_{2}$}

During hot starting, $\mathrm{CO}_{2}$ emissions increase rapidly as the engine is started, before reaching typical $13.8 \%$ concentration levels. Likewise, $\mathrm{O}_{2}$ emissions decay rapidly as the engine is started, down to typical $1 \%$ concentration levels. No major differences for the different test fuels were seen for either $\mathrm{CO}_{2}$ or $\mathrm{O}_{2}$ emissions. 


\section{Chapter 8}

\section{Conclusions and Recommendations}

Previous work on this gasoline direct injection (GDI) engine showed a high degree of variability of particulate matter (PM) emissions during otherwise fully warmed, steady-state operation. PM emissions were shown to vary not only on a run-to-run basis, but also in a temporal "drift" with run-time. Turning to the literature identified several possible systematic sources of these variations, along with the potential influence of fuel composition.

Therefore, the objectives of this investigation were to identify and control variabilities associated with the production of PM from this GDI engine, at steady-state operation. Additional objectives were to provide commentary on the suitability of ethanol as a PM control strategy, to characterize emissions during transient starting procedures, and to compare the results provided by different gaseous instruments and mass determination techniques. The following conclusions and recommendations are made based on these results.

\subsection{Conclusions}

The major conclusions from this work are outlined in the following subsections, grouped by the major objectives.

\subsubsection{Sources of PM Emission Variability}

\section{Systematic Sources}

Several systematic sources of variability were identified and tested at the onset of this study. Using a dry dilution air supply for the diluter (Rotating Disk Thermodiluter), used with the particle sizer (EEPS) instead of ambient air in its original configuration, reduced the test-to-test variability of particle number (PN) concentrations substantially; a 54\% reduction in relative standard deviations (RSD) was seen. The stability of the dilution air humidity provided by this system, rather than the dryness of the air itself, was thought to be the main reason for this finding.

Using a fuel cooler to stabilize fuel temperatures to ambient conditions, and an oil cooler to lower steady-state oil temperatures, proved non-beneficial from a variability stand point. Closer inspection showed that use of the oil cooler resulted in an increase in PM emissions, likely due to lowering the in-cylinder surface temperatures (piston crown), thereby reducing the evaporation of impinged fuel. 
Removing induction system (air-intake) deposits, and preventing their formation via the ingress of oil from the crankcase ventilation system with a coalescing filter (PCV filter), initially seemed to have addressed both the issues of run-to-run variability and temporal variation; this was later determined not to be the case. Visual inspection of the injector faces over this investigation showed no appreciable change in deposit level, leading to the conclusion that this was also not the source of changing emissions levels. The final parameter investigated was the influence of engine oil age. These tests showed no systematic variation in PM emissions as mileage was accumulated after an oil change.

To summarize, the investigation into systematic sources of variability provided very little to no relief from the observed variability. Aside from the dilution air, the changes observed were largely at a lower scale than the variabilities in question.

\section{Fuel Composition}

Varying the fuel composition resulted in a high degree of PM emissions variability. Generally speaking, increasing the ethanol or toluene proportions of the fuel blend increased measured PN concentrations; relative standard deviation (RSD) values also increased with increasing fractions, indicating a decrease in repeatability. Increasing both fractions together exacerbated PM emissions further leading, to the conclusion that the high latent heat of vaporization of ethanol causes poorer fuel-air mixing and leads to favourable sooting conditions for toluene. Emissions variabilities were also seen at the base fuel (gasoline) level. Two groups of tests using gasoline fuel (E0) showed wildly different results; average PN concentrations differed by a factor of two, while run-to-run repeatability (RSD) varied by an approximate factor of 1.6.

PM mass results displayed linear correlations with PN concentrations, mirroring those found in the literature. In this study, gasoline-ethanol blends provided a correlation of $\sim 1.43 \times 10^{12} \mathrm{\#} / \mathrm{mg}$. Toluene based fuel blends appeared to emit more mass at fixed PN concentrations, providing a less steep correlation of $\sim 1.09 \times 10^{12} \# / \mathrm{mg}$. This was corroborated by the particle size distributions, where the toluene blended fuels showed shifts to larger sizes with increases to the modal and median diameters. In terms of composition, increasing either the ethanol or toluene fractions caused an increase in measured $\mathrm{EC} / \mathrm{OC}$ ratios; i.e. a greater elemental fraction. EC/OC ratios for gasoline (E0), E10, and T10 were less then one, while E30 and T10E10 provided ratios greater than one. Inspection of elemental and organic concentrations shows that this arose from increases in the elemental portion of the PM, while the organic concentration remained largely fixed. The conclusion drawn from this observation was that fundamental changes to the combustion process had occurred.

Inspection of gas phase emissions showed that PN concentration differences trended with three compounds; toluene, ethanol, and isobutylene. Correlating these three compounds in linear least-squares regressions with measured PN concentrations, provided good agreement for E0 and E10 fuel blend data, including a commercially available E10 fuel (Fuel A). Increasing toluene exhaust concentrations correlated with increasing PN concentrations, while increasing isobutylene exhaust concentrations showed a negative correlation; ethanol provided for step-change increases in PN concentrations. Coefficients of a multiple linear regression (MLR) showed statistical significance at the $99 \%$ confidence level, leading to the conclusion that these three compounds portray an accurate depiction of the measured PN concentrations, including their variabilities. Literature-derived PM indices for fuel composition, which correlated the sooting propensity (DBE) of a fuel and its vapour pressure as key identifiers of PM production, provided physical interpretation of the MLR results. Using these indices as a guide, it was confirmed that 
the correlation with isobutylene concentrations provided an adequate marker for the vapour pressure changes of the base gasoline fuel, while the toluene accounted for DBE changes. Both of these factors are commonly modified in the production of gasoline to meet seasonal and octane rating requirements.

Therefore, it was postulated that changes to the commercially obtained gasoline fuel (used as the base for all the fuel blends here) had occurred over the course of the investigation. This was confirmed when comparing the two gasoline (E0) fuelled test groups; tests in the group with lower PN concentrations emitted substantially lower toluene and higher isobutylene concentrations than the tests in the group with higher PM emissions. As further evidence, correcting the theoretical indices provided by literature as a first approximation for the changing base fuel composition accounted for the PN concentration changes more clearly

\section{Overall Conclusion}

The primary takeaway from this work can be summarized as the following: changes in composition of the base gasoline fuel can cause wide variations in measured PM emissions, both in mass and number of particles.

This has wide ranging implications for GDI engine development, as commercial gasoline fuels could be curtailed for low PM production with the careful consideration of constituents. Furthermore, the fact that vehicle emissions certification is performed with tightly controlled fuel compositions - for good reasonraises the question of whether these vehicles still meet the emissions targets in real world application. From the data available, the use of ethanol proves counter productive for reducing PM emission at highway cruise conditions. However, due to the inherent base fuel variabilities encountered here, more work is required to enable a definitive conclusion on the efficacy of ethanol as reduction strategy.

\subsubsection{Additional Conclusions}

Auxiliary analysis of transient engine operation and instrument comparisons provides for the secondary set of conclusions, which follow below.

\section{Transient Emissions Behaviour}

Increasing ethanol concentrations showed positive reductions in cumulative PM emissions during both cold and hot starts, with E30 showing the greatest reductions. This was attributed to the oxygen availability in the ethanol molecule which aids the oxidation of un-burned hydrocarbon species and soot precursors during the combustion process. Increasing toluene to the $10 \%$ by volume level showed no real difference at both cold and hot start conditions; however, when ethanol and toluene were combined at the 10\% level (T10E10) PM emissions were exacerbated. This follows the observations at steady-state engine operation and so the same conclusion is applied; increasing the proportion of ethanol provides for greater inhomogeneities of the fuel charge due to vaporization issues, which results in a greater net emission due to the increased availability of toluene for PM production.

Cold start tests were dominated by nucleation mode size particles, while hot start tests showed accumulation mode dominated size distributions. Compositional analysis showed emissions to be dominated by the organic fraction at both conditions; increasing the ethanol tended to increase the organic frac-

tion. The opposite was seen at highway cruise conditions, indicating that sufficient fuel-air mixture preparation is likely a larger issue at slow and cold engine operation. 
Therefore, unlike the highway cruise condition results, the addition of ethanol does provide for discernible PM reductions during transient starting operations.

\section{Instrument Comparisons:}

\section{Gaseous Emissions Measurement}

Comparing the two gaseous emissions analyzers (emissions bench NDIR vs. FTIR) shows that measured $\mathrm{CO}_{2}$ and $\mathrm{CO}$ concentrations from the emissions bench NDIR were typically $7 \%$ and $2 \%$ higher than FTIR average concentrations, respectively. In contrast, emissions bench $\mathrm{NO}_{\mathrm{x}}$ measurements were systematically lower by $5 \%$ over the measured FTIR levels. These values compared favourably with those listed in the literature and lead to the conclusion that the FTIR does a good job at measuring standard compounds; the emissions bench NDIR is currently the approved method for gaseous emissions measurement according to the US EPA.

\section{Mass Determination Measurement}

Both the EC/OC analysis and gravimetric analysis of PM laden filters provide an estimation of the solid carbon emissions from the engine. In this case, elemental concentrations of PM given by the EC/OC analysis were approximately $30 \%$ higher on average when compared to the gravimetric derived concentrations. The hypothesis offered here is that more refinement of the EC/OC analysis method is required to fully account for the organic fraction. Furthermore, issues with sample handling could also account for the observed differences, as could evaporative losses of lighter hydrocarbon species from the gravimetric measurements.

\subsection{Recommendations}

Difficulties encountered during this work have presented several avenues for improvements. These improvements are listed below and include systematic changes to the apparatus, along with changes to current methodology.

\section{Apparatus Modifications}

- The internal heated lines for the emissions bench are currently limited to $150{ }^{\circ} \mathrm{C}$ by design. These should be replaced with heated lines capable of heating up to the required $191{ }^{\circ} \mathrm{C}$ for gaseous sampling.

- Move the thermocouples on the filter collection cart closer to the filter cartridges to provide more accurate filter temperature readings, in order to maintain the required temperature of $47 \pm 5{ }^{\circ} \mathrm{C}$.

- Reconfigure the dynamometer wiring harness to provide power to the PCM regardless of the ignition position selection. This will allow conclusions to be made regarding the learning capabilities of the PCM (LTFT) and will provide more information regarding emissions variabilities.

- Acquire the necessary DAQ capabilities to accurately measure important engine parameters, such as valve timing, injection timing and duration, and spark timing. This will provide much needed insight to the base calibration that the PCM employs. 
- Incorporate intake air humidity measurements into the current data stream to appropriately correct $\mathrm{NO}_{\mathrm{x}}$ emission measurements in real-time, and to observe any humidity dependence on PM formation from this engine. This could also account for the current $5 \%$ difference in $\mathrm{NO}_{\mathrm{x}}$ between the FTIR and the emissions bench NDIR.

- Modify the research engine to provide access for in-cylinder pressures (pressure transducer) to calculate IMEP values. This will permit a more conclusive commentary on changing combustion stability with changing fuel composition.

- Re-range the filter collection cart mass flow controllers to $70 \mathrm{lpm}$ to permit face velocities of 100 $\mathrm{cm} / \mathrm{s}$, per US EPA protocols. Currently the flow controllers are limited to $35 \mathrm{lpm}$.

\section{Methodology Changes}

- Implement a basic drive-cycle consisting of transient and steady-state procedures linked together using the procedures developed here and in previous work on this engine. This will provide not only a more realistic driving simulation from an emissions perspective, but also the ability to collect meaningful gravimetric samples during transient driving phases.

- Expand the compositional analysis repertoire to include metals analysis on PM laden filters. This will require the collection of samples onto Zeflour ${ }^{\mathrm{TM}}$ filters which can be cut into appropriate sample sizes.

\subsection{Future Work}

Performing the above recommendations will permit a more thorough undertaking for the future work on this project. Quite simply, the issue of fuel variability needs to be explored further. This can be done by the blending of pseudo-certification fuels with tightly controlled constituent fractions and judging whether the PM emissions variability is reduced. Additionally, this can apportion the relative influences of both the DBE and vapour pressure on the PM emissions. This will provide the evidence required to confidently say that the PM emissions variability experienced with this engine are due to commercial fuel variances, and by extension, will allow for a re-evaluation of whether the use of commercial fuels in this type of research is appropriate. The implementation of a drive cycle will provide additional information on the impact of fuel variability under differing conditions, thus giving a better outlook on emissions from this engine.

The conclusion that increasing ethanol causes increased PM emissions at highway cruise conditions also needs further investigation. Several hypotheses have been provided here as possible explanations from an engine behavioural perspective. The idea that increased wall-impingement from ethanol blended fuels due to vaporization issues could be explored by using a biologically derived lubrication oil as a marker for oil ingress into the combustion process. These bio-oils would then provide a pathway for carbon-13 tracing with mass spectroscopy. In the same vein, the aforementioned modification to the wiring harness, which would provide power to the PCM at all times, would allow an avenue to analyze the impact that learning has on PM emissions stabilization. Elimination of these will likely infer that the changes must be occurring at the combustion process level.

Continuing this project on its original trajectory - that is, the exploration of ethanol as a control technique for PM emissions - will require an expansion of the current test fuel matrix to include higher 
level oxygenates. Currently, 30\% by volume (E30) represents the maximum amount that has been tested on this engine. Performing tests at E85 will be particularly interesting given its prevalence in certain markets (USA and Brazil) and may prove helpful in determining its usefulness in Canada. 


\section{References}

[1] Environment Canada, "Renewable Fuels Regulations," Canada Gazette Part II, SOR/2010-189, 2010.

[2] Environment Canada, "On-Road Vehicle and Engine Emissions Regulations," Canada Gazette Part II, SOR/2003-2, 2003.

[3] Environment Canada, "Passenger Automobile and Light Truck Greenhouse Gas Emission Regulations," Canada Gazette Part II, SOR/2010-201, 2010.

[4] T. W. Chan, E. Meloche, J. Kubsh, R. Brezny, D. Rosenblatt, and G. Rideout, "Impact of Ambient Temperature on Gaseous and Particle EMissions from a Direct Injection Gasoline Vehicle and its Implications on Particle Filtration," SAE Technical Paper Series, Tech. Rep. 2013-01-0527, 2013.

[5] F. Zhao, M. C. Lai, and D. L. Harrington, "Automotive spark-ignited direct-injection gasoline engines," Progress in Energy and Combustion Science, vol. 25, no. 5, pp. 437 - 562, 1999. [Online]. Available: http://www.sciencedirect.com/science/article/pii/S0360128599000040

[6] P. Price, R. Stone, T. Collier, and M. Davies, "Particulate Matter and Hydrocarbon Emissions Measurements: Comparing First and Second Generation DISI with PFI in Single Cylinder Optical Engines," SAE Technical Paper Series, Tech. Rep. 2006-01-1263, 2006.

[7] S. Zhang and W. McMahon, "Particulate Emissions for LEV II Light-Duty Gasoline Direct Injection Vehicles," SAE Technical Paper Series, Tech. Rep. 2012-01-0442, 2012.

[8] J. B. Heywood, Internal Combustion Engine Fundamentals. New York, NY: McGraw-Hill Inc., 1988.

[9] M. C. Drake, T. D. Fansler, A. S. Solomon, and G. A. Szekely, Jr., "Piston Fuel Films as a Source of Smoke and Hydrocarbon Emissions from a Wall-Controlled Spark-Ignited Direct-Injection Engine," SAE Technical Paper Series, Tech. Rep. 2003-01-0547, 2003.

[10] T. W. Chan, E. Meloche, J. Kubsh, D. Rosenblatt, R. Brezny, and G. Rideout, "Evaluation of a Gasoline Particulate Filter to Reduce Particle Emissions from a Gasoline Direct Injection Vehicle," SAE Technical Paper Series, Tech. Rep. 2012-01-1727, 2012.

[11] C. A. Pope III and D. W. Dockery, "Health Effects of Fine Particulate Air Pollution: Lines that Connect," Air \& Waste Management Association, vol. 56, pp. 709 - 742, 2006.

[12] C. A. Pope III, M. Ezzati, and D. W. Dockery, "Fine-Particulate Air Pollution and Life Expectancy in the United States," The New England Journal of Medicine, vol. 360, no. 4, pp. 376 - 386, 2009. 
[13] R. D. Brook, S. Rajagopalan, C. A. Pope III, J. R. Brook, A. Bhatnagar, A. V. Diez-Roux, F. Holguin, Y. Hong, R. V. Leupker, M. A. Mittleman, A. Peters, D. Siscovick, S. C. Smith, L. Whitsel, and J. D. Kaufman, "Particulate Matter Air Pollution and Cardiovascular Disease: An Update to the Scientific Statement from the American Heart Association," American Heart Association, vol. 121, pp. 2331 - 2378, 2010. [Online]. Available: http://circ.ahajournals.org/content/121/21/2331

[14] L. Benbrahim-Tallaa, R. A. Baan, Y. Grosse, B. Lauby-Secretan, F. El Ghissassi, V. Bouvard, N. Guha, D. Loomis, and K. Straif, "Carcinogenicity of diesel-engine and gasoline-engine exhausts and some nitroarenes," Lancet Oncology, vol. 13, pp. 663 - 664, 2012.

[15] T. Johnson, "Vehicular Emissions in Review," SAE Technical Paper Series, Tech. Rep. 2013-010538, 2013.

[16] T. L. Barone, J. M. E. Storey, A. D. Youngquist, and J. P. Szybist, "An analysis of direct-injection spark-ignition (DISI) soot morphology," Atmospheric Environment, vol. 49, pp. 268 -274, 2012.

[17] L. A. Sgro, P. Sementa, B. M. Vaglieco, G. Rusciano, A. D'Anna, and P. Minutolo, "Investigating the origin of nuclei particles in GDI engine exhausts," Combustion and Flame, vol. 159, pp. 1687 $-1692,2012$.

[18] M. M. Maricq, "Soot Formation in ethanol/gasoline fuel blend diffusion flames," Combustion and Flame, vol. 159, pp. 170 - 180, 2012.

[19] J. Serras-Pereira, P. Aleiferis, D. Richardson, and S. Wallace, "Spray Development, Flow Interactions and Wall Impingement in a Direct-Injection Spark-Ignition Engine," SAE Technical Paper Series, Tech. Rep. 2007-01-2712, 2007.

[20] H. Oh, C. Bae, and K. Min, "Spray and Combustion Characteristics of Ethanol Blended Gasoline in a Spray Guided DISI Engine under Lean Stratified Operation," SAE Technical Paper Series, Tech. Rep. 2010-01-2152, 2010.

[21] A. Warey, Y. Huang, R. Matthews, M. Hall, and H. Ng, "Effects of Piston Wetting on Size and Mass of Particulate Matter Emissions in a DISI Engine," SAE Technical Paper Series, Tech. Rep. 2002-01-1140, 2002.

[22] L. Chen, R. Stone, and D. Richardson, "A study of mixture preparation and PM emissions using a direct injection engine fuelled with stoichiometric gasoline/ethanol blends," Fuel, vol. 96, pp. 120 $-130,2012$.

[23] L. Chen, R. Stone, and D. Richardson, "Effect of the valve timing and the coolant temperature on particulate emissions from a gasoline direct-injection engine fuelled with gasoline and with a gasoline-ethanol blend," Proceedings of the Institution of Mechanical Engineers, Part D: Journal of Automobile Engineering, vol. 226, no. 10, pp. 1419 - 1430, 2012.

[24] I. Dimou, K. Kar, and W. Cheng, "Particulate Matter Emissions from a Direct Injection Spark Ignition Engine under Cold Fast Idle Conditions for Ethanol-Gasoline Blends," SAE Technical Paper Series, Tech. Rep. 2011-01-1305, 2011. 
[25] S. Samuel, A. Hassaneen, and D. Morrey, "Particulate Matter Emissions and the Role of Catalytic Converter During Cold Start of GDI Engine," SAE Technical Paper Series, Tech. Rep. 2010-012122,2010 .

[26] I. A. Khalek, T. Bougher, and J. J. Jetter, "Particle Emissions from a 2009 Gasoline Direct Injection Engine Using Different Commercially Available Fuels," SAE Technical Paper Series, Tech. Rep. 2010-01-2117, 2010.

[27] M. M. Maricq, D. H. Podsiadlik, D. D. Brehob, and M. Haghgooie, "Particulate Emissions from a Direct-Injection Spark-Ignition (DISI) Engine," SAE Technical Paper Series, Tech. Rep. 1999-011530, 1999.

[28] P. Price, B. Twiney, R. Stone, K. Kar, and H. Walmsley, "Particulate and Hydrocarbon Emissions from a Spray Guided Direct Injection Spark Ignition Engine with Oxygenate Fuel Blends," SAE Technical Paper Series, Tech. Rep. 2007-01-0472, 2007.

[29] X. He, J. C. Ireland, B. T. Zigler, M. A. Ratcliff, K. E. Knoll, T. L. Alleman, J. H. Luecke, and J. T. Tester, "The Impacts of Mid-Level Alcohol Content in Gasoline on SIDI Engine-Out and Tailpipe Emissions," in Proceedings of the ASME 2010 Internal Combustion Engine Division Fall Technical Conference, no. ICEF2010-35106. ASME, September.

[30] S. Sakai, M. Hageman, and D. Rothamer, "Effect of Equivalence Ratio on the Particulate Emissions from a Spark-Ignited, Direct-Injected Gasoline Engine," SAE Technical Paper Series, Tech. Rep. 2013-01-1560, 2013.

[31] I. Whelan, S. Samuel, and A. Hassaneen, "Investigation into the Role of Catalytic Converters on Tailpipe-out Nano-Scale Particulate Matter from Gasoline Direct Injection Engine," SAE Technical Paper Series, Tech. Rep. 2010-01-1572, 2010.

[32] T. Wallner and S. A. Miers, "Combustion Behavior of Gasoline and Gasoline/Ethanol Blends in a Modern Direct-Injection 4-Cylinder Engine," SAE Technical Paper Series, Tech. Rep. 2008-010077, 2008.

[33] K. Kar, R. Tharp, M. Radovanovic, I. Dimou, and W. K. Cheng, "Organic gas emissions from a stroichiometric direct injection spark ignition engine operating on ethanol/gasoline blends," International Journal of Engine Research, vol. 11, no. 6, pp. 449 - 513, 2010.

[34] T. Wallner and R. Frazee, "Study of Regulated and Non-Regulated Emissions from Combustion of Gasoline, Alcohol Fuels and their Blends in a DI-SI Engine," SAE Technical Paper Series, Tech. Rep. 2010-01-1571, 2010.

[35] G. Broustail, F. Halter, P. Seers, e. G. Mor and C. Mounaim-Rousselle, "Comparison of regulated and non-regulated pollutants with iso-octane/butanol and iso-octane/ethanol blends in a port-fuel injection Spark-Ignition engine," Fuel, vol. 94, pp. 251 - 261, 2012.

[36] G. Karavalakis, T. D. Durbin, M. Shrivastava, Z. Zheng, M. Villela, and H. Jung, "Impacts of ethanol fuel level on emissions of regulated and unregulated pollutants from a fleet of gasoline light-duty vehicles," Fuel, vol. 93, pp. 549 - 558, 2012. 
[37] L. A. Graham, S. L. Belisle, and C.-L. Baas, "Emissions from light duty gasoline vehicles operating on low blend ethanol gasoline and E85," Atmospheric Environment, vol. 42, pp. 4498 - 4516, 2008.

[38] S. Poulopoulos, D. Samaras, and C. Philippopoulos, "Regulated and unregulated emissions from an internal combustion engine operating on ethanol-containing fuels," Atmospheric Environment, vol. 35, pp. $4399-4406,2001$.

[39] M. Koç, Y. Sekmen, T. Topgül, and H. Yücesu, "The effects of ethanol-unleaded gasoline blends on engine performance and exhaust emissions in a spark-ignition engine," Renewable Energy, vol. 34, pp. $2101-2106,2009$.

[40] United States Environmental Protection Agency, "40 CFR 1065.130 - Engine exhaust," Government Printing Office, 2012.

[41] United States Environmental Protection Agency, "40 CFR 1065.170 - Batch sampling for gaseous and PM constituents," Government Printing Office, 2012.

[42] M. M. Maricq, J. Szente, M. Loos, and R. Vogt, "Motor Vehicle PM Emissions Measurement at LEV III Levels," SAE Technical Paper Series, Tech. Rep. 2011-01-0623, 2011.

[43] J. Bushkuhl, W. Silvis, J. Szente, and M. Maricq, "A New Approach for Very Low Particulate Mass Emissions Measurement," SAE Technical Paper Series, Tech. Rep. 2013-01-1557, 2013.

[44] P. Mireault, "Methodology of Measuring Particulate Matter Emissions from a Gasoline Direct Injection Engine," Master's thesis, University of Toronto, January 2014.

[45] T. Rönkkö, A. Virtanen, K. Vaaraslahti, J. Keskinen, L. Pirjola, and M. Lappi, "Effect of dilution conditions and driving parameters on nucleation mode particles in diesel exhaust: Laboratory and on-road study," Atmospheric Environment, vol. 40, pp. 2893-2901, 2006.

[46] J. P. Shi and R. M. Harrison, "Investigation of ultrafine particle formation during diesel exhaust dilution," Environmental Science \&3 Technology, vol. 33, no. 21, pp. 3730-3736, 1999.

[47] D. Kittelson and I. Abdul-Khalek, "Formation of nanoparticles during exhuast dilution," in Fuels, Lubricants, Engines, \& Emissions. EFI Members Conference, January 1999.

[48] A. Berndorfer, S. Breuer, W. Piock, and P. V. Bacho, "Diffusion Combustion Phenomena in GDi Engines caused by Injection Process," SAE Technical Paper Series, Tech. Rep. 2013-01-0261, 2013.

[49] O. Berkemeier, K. Grieser, K. Hohenboeken, E. Karvounis, and K. M. Springer, "Strategies to Control Particulate Emissions of Gasoline Direct Injection Engines," in Proceedings of the FISITA 2012 World Automotive Congress, vol. 189, 2013, pp. 699-714.

[50] P. W. Guthrie, "A Review of Fuel, Intake and Combustion System Deposit Issues Relevant to 4-Stroke Gasoline Direct Fuel Injection Engines," SAE Technical Paper Series, Tech. Rep. 200101-1202, 2001.

[51] D. W. Mackney, R. M. Calder, M. G. J. Macduff, and M. W "Reducing Deposits in a DISI Engine," Tech. Rep. 
[52] P. S. V. Bacho, J. K. Sofianek, J. M. Galante-Fox, and C. J. McMahon, "Engine Test for Accelerated FUel Deposit Formation on Injectors Used in Gasoline Direct Injection Engines," SAE Technical Paper Series, Tech. Rep. 2009-01-1495, 2009.

[53] M. Russell, J. Cummings, T. Cushing, and W. Studzinkski, "Cellulosic Ethanol Fuel Quality Evaluation and its Effects on PFI Intake Valve Deposits and GDI Fuel Injector Pluggin Performance," SAE Technical Paper Series, Tech. Rep. 2013-01-0885, 2013.

[54] F. Parsinejad and W. Biggs, "Direct Injection Spark Ignition Engine Deposit Analysis: Combustion Chamber and Intake Valve Deposits," SAE Technical Paper Series, Tech. Rep. 2011-01-2110, 2011.

[55] N. Arnault and S. Bonne, "Engine Lube-Oil Consumption Stakes and Benefits from Significant Blow-by Oil Mist Reduction," SAE Technical Paper Series, Tech. Rep. 2012-01-1617, 2012.

[56] I. Whelan, S. Samuel, and A. Hassaneen, "The Effect of Fuel Temperature on Particulate Matter Formation in Gasoline Direct-Injection Engines," SAE Technical Paper Series, Tech. Rep. 201001-1469, 2010.

[57] M. G. Christianson, E. Bardasz, and W. Nahumck, "Impact of Lubricating Oil Condition on Exhaust Particulate Matter Emissions from Light Duty Vehicles," SAE Technical Paper Series, Tech. Rep. 2010-01-1560, 2010.

[58] P. Kapus, A. Fuerhapter, H. Fuchs, and G. K. Fraidl, "Ethanol Direct Injection on Turbocharged SI Engines - Potential and Challenges," SAE Technical Paper Series, Tech. Rep. 2010-01-1560, 2010.

[59] T. Sagawa, H. Fujimoto, and K. Nakamura, "Study of Fuel Dilution in Direct-Injection and Multipoint Injection Gasoline Engines," SAE Technical Paper Series, Tech. Rep. 2002-01-1647, 2002.

[60] C. Vuk and S. J. Vander Griend, "Fuel Property Effects on Particulates In Spark Ignition Engines," SAE Technical Paper Series, Tech. Rep. 2013-01-1124, 2013.

[61] J. M. Storey, T. Barone, K. Norman, and S. Lewis, "Ethanol Blend Effects On Direct Injection Spark-Ignition Gasoline Vehicle Particulate Matter Emissions," SAE Technical Paper Series, Tech. Rep. 2010-01-2129, 2010.

[62] Y. Kim, Y. Kim, S. Y. Jun, S. H. Rew, D. Lee, and S. Park, "Fuel Effect on Particle Emissions of a Direct Injection Engine," SAE Technical Paper Series, Tech. Rep. 2013-01-1559, 2013.

[63] K. Aikawa, T. Sakurai, and J. J. Jetter, "Development of a Predictive Model for Gasoline Vehichle Particulate Matter Emissions," SAE Technical Paper Series, Tech. Rep. 2010-01-2115, 2010.

[64] F. Leach, R. Stone, and D. Richardson, "The Influence of Fuel Properties on Particulate Number Emissions from a Direct Injection Spark Ignition Engine," SAE Technical Paper Series, Tech. Rep. 2013-01-1558, 2013.

[65] Ford Motor Company, "2012 Ford Focus Technical Specifications," 2012. [Online]. Available: http://media.ford.com/images/10031/2012_Focus_Specs.pdf 
[66] MANN+HUMMEL, "MANN+HUMMEL ProVent: Oil separator for closed and open crankcase ventilation," 2013. [Online]. Available: https://www.mann-hummel.com/fileadmin/user_upload/ service/catalogues/pdf/ProVent_en_2013.pdf

[67] ECM: Engine Control and Monitoring, "appsCAN Module and gpioCAN Module Instruction Manual," Los Altos, California, 2009.

[68] TSI Incorporated, "Sampling, Diluting and Conditioning for Characterizing Particle Emissions - Thermodiluter and Thermal Conditioner," 2007. [Online]. Available: http://www.tsi.com/ uploadedFiles/_Site_Root/Products/Literature/Brochures/379020-30thermodiluters.pdf

[69] Dekati Ltd., "Dekati FPS-4000 Fine Particle Sampler," Kangasala, Finland, 2014. [Online]. Available: http://s3.amazonaws.com/dekati_s3/attachments/000/000/466/original/ 9fd85e50a0eace41b3cb74cc5d07cd24

[70] J. Ketterer, "Effects of biodiesel fuelling on diesel particulate matter," Master's thesis, University of Toronto, 2011.

[71] C. Wan, "Effects of biodiesel fuelling on diesel particulate matter," Master's thesis, University of Toronto, 2011.

[72] TSI Incorporated, "Engine Exhaust Particle Sizer Spetrometer Model 3090," 2012. [Online]. Available: http://www.tsi.com/uploadedFiles/_Site_Root/Products/Literature/Spec_ Sheets/3090_2980244A.pdf

[73] United States Environmental Protection Agency, "40 CFR 1065.140 - Dilution for gaseous and PM constituents," Government Printing Office, 2012.

[74] Environmental and Industrial Sciences Division RTI International, "Standard Operating Procedure for Particulate Matter (PM) Gravimetric Analysis," United States Environmental Protection Agency, 2008.

[75] Sunset Laboratory Inc., "Semi-Continuous OCEC Carbon Aerosol Analyzer - A guide to running and maintaining the Sunset Laboratory semi-continous OCEC analyzer," 2006.

[76] Pall Corporation, "PTFE Membrane Disc Filters," 2014. [Online]. Available: http: //www.pall.com/main/laboratory/product.page?id=20061

[77] Pall Corporation, "Pallflex@Air Monitoring Filters," 2014. [Online]. Available: http: //www.pall.com/main/laboratory/product.page?id=20056

[78] ECM: Engine Control and Monitoring, "AFRecorder 2400," Los Altos, California, 2011. [Online]. Available: http://ecm-co.com/product.asp?2400

[79] United States Environmental Protection Agency, "40 CFR 1065.655 - Chemical balances of fuel, intake air, and exhaust," Government Printing Office, 2014.

[80] MKS Instruments: On-Line Product Group, "User Training MultiGas 2030 FT-IR Gas Analysis," Massachusetts, 2011. 
[81] United States Environmental Protection Agency, "40 CFR 1065.260 - Flame-ionization detector," Government Printing Office, 2014.

[82] CAI: California Analytical, "CAI 600 Series HFID User's Manual," Orange, California, 2011. [Online]. Available: http://www.gasanalyzers.com/uploads/product/product_manual/16/ 600_SERIES_HFID_OPS_MANUAL_214.pdf

[83] United States Environmental Protection Agency, "40 CFR 1065.270 - Chemiluminescent detector," Government Printing Office, 2014.

[84] CAI: California Analytical, "CAI 600 Series HCLD User's Manual," Orange, California, 2011. [Online]. Available: http://www.gasanalyzers.com/uploads/product/product_manual/24/ 600_SERIES_HCLD_OPERATORS_MANUAL_1213.pdf

[85] United States Environmental Protection Agency, "40 CFR 1065.250 - Nondispersive infrared analyzer," Government Printing Office, 2014.

[86] United States Environmental Protection Agency, "40 CFR 1065.280 - Paramagnetic and magnetopneumatic O2 detection analyzers," Government Printing Office, 2014.

[87] CAI: California Analytical, "CAI 600 Series NDIR/O2 User's Manual," Orange, California, 2011. [Online]. Available: http://www.gasanalyzers.com/uploads/product/product_manual/13/ 600_SERIES_NDIR_OPERATORS_MANUAL_REV_1213.pdf

[88] LI-COR Biosciences, "LI-840A CO2/H20 Gas Analyzer: Instruction Manual," Licoln, Nebraska, 2013. [Online]. Available: ftp://ftp.licor.com/perm/env/LI-840A/Manual/LI-840A_Manual.pdf

[89] Ford Motor Company, "2012 Focus/Focus BDV/Focus ST Service Information CD-ROM," CDROM, 2011.

[90] F. Catapano, S. Di Iorio, M. Lazzaro, P. Semenaa, and B. M. Vaglieco, "Characterization of Ethanol Blends Combustion Processes and Soot Formation in a GDI Optical Engine," SAE Technical Paper Series, Tech. Rep. 2013-01-1316, 2013.

[91] X. He, M. A. Ratcliff, and B. T. Zigler, "Effects of Gasoline Direct Injection Engine Operating Parameters on Particle Number Emissions," Energy \&3 Fuels, vol. 26, pp. 2014 - 2027, 2012.

[92] M. M. Maricq, J. J. Szente, and K. Jahr, "The Impact of Ethanol Fuel Blends on PM Emissions from a Light-Duty GDI Vehicle," Aerosol Science and Technology, vol. 46, pp. 576-583, 2012. [Online]. Available: http://www.tandfonline.com/doi/abs/10.1080/02786826.2011.648780

[93] I. Glassman and R. A. Yetter, Combustion, 4th ed. New York: Elsevier Inc., 2008.

[94] C. Arcoumanis and T. Kamimoto, Eds., Flow and Combustion in Reciprocating Engine. Berlin: Springer, 2009.

[95] United States Environmental Protection Agency, "40 CFR 79.55 - Base Fuel Specifications," Government Printing Office, 2014.

[96] United States Environmental Protection Agency, "40 CFR 80.27 - Control and Prohibition on Gasoline Volatility," Government Printing Office, 2014. 
[97] S. R. Turns, An Intro to Combustion: Concepts and Applications, 2nd ed. Toronto: McGraw-Hill Companies, Inc., 2000.

[98] Continental Tire, "ContiProContact ${ }^{T M}$ Datasheet," 2013. [Online]. Available: http://www.conti-online.com/generator/www/ca/en/continental/automobile/themes/pcar/ allseason/hidden/datasheet-procontact_en.pdf

[99] Sea Foam Sales Company, "Sea foam motor treatment: Material safety data sheet," Eden Prairie, Minnesota, 2013. [Online]. Available: http://seafoamsales.com/wp-content/uploads/2012/12/ MSDS_seafoam_EN.pdf

[100] K-G Spray-Pak INC., "Motomaster carburetor cleaner: Material safety data sheet," Concord, Ontario, 2012. [Online]. Available: http://msds.canadiantire.ca/OpenRepositoryFile.asp?s= $164664 \& v=3$

[101] W. B. Ribbens, Understanding Automotive Electronics (Sixth Edition), 6th ed., W. B. Ribbens, N. P. Mansour, G. Luecke, C. W. Battle, E. C. Jones, and L. E. Mansir, Eds. Burlington: Newnes, 2003.

[102] L. Chen and R. Stone, "Measurement of Enthalpies of Vaporization of Isooctane and Ethanol Blends and Their Effects on PM Emissions from a GDI Engine," Energy and Fuel, vol. 25, no. 3, pp. 1254 - 1259, 2011. [Online]. Available: http://dx.doi.org/10.1021/ef1015796

[103] J. Serras-Pereira, P. G. Aleiferis, and D. Richardson, "Imaging and heat flux measurements of wall impinging sprays of hydrocarbons and alcohols in a direct-injection spark-ignition engine," Fuel, vol. 91, pp. $264-297,2012$.

[104] United States Environmental Protection Agency, "Emission Factors \& AP 42: Organic Liquid Storage Tanks," 2006. [Online]. Available: http://www.epa.gov/ttn/chief/ap42/ch07/ final/c07s01.pdf 


\section{Appendix A}

\section{Chapter 4 Supplementary Apparatus Information}

\section{A.1 Pro-Vent 200 Modification}

Engineering diagram for "plug" to seal malfunctioning regulation valve on the PCV filter. 


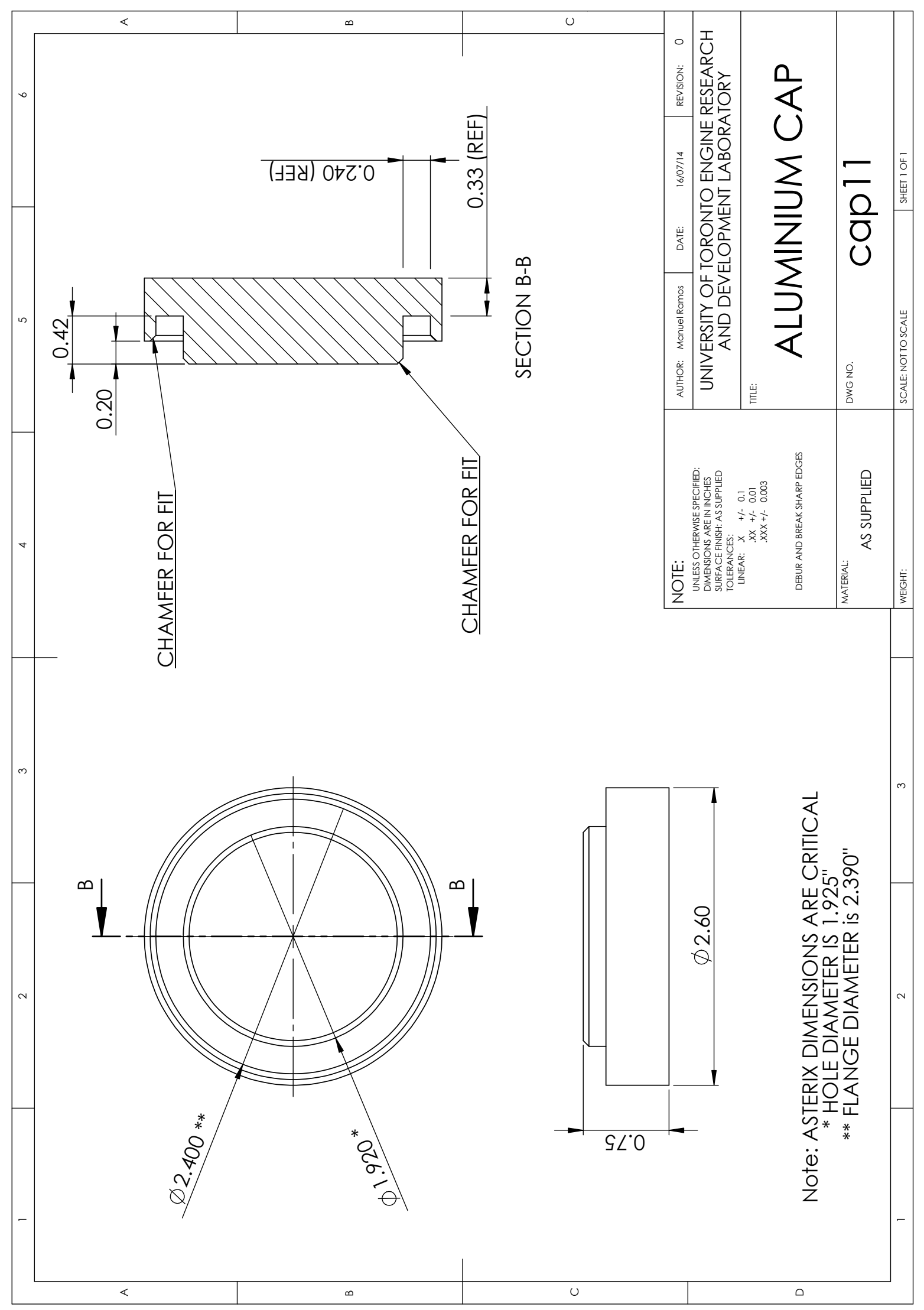




\section{A.2 Atomic Ratio Calculations}

Calculations for the atomic ratio values presented in Table 4.5.

\section{A.2.1 Ethanol Content Regression}

Regressions for the $\mathrm{H}: \mathrm{C}$ and $\mathrm{O}: \mathrm{C}$ ratios used to determine missing atomic coefficients are contained in Figure A.1. Atomic coefficients used to generate the above regressions are contained in Table A.1.

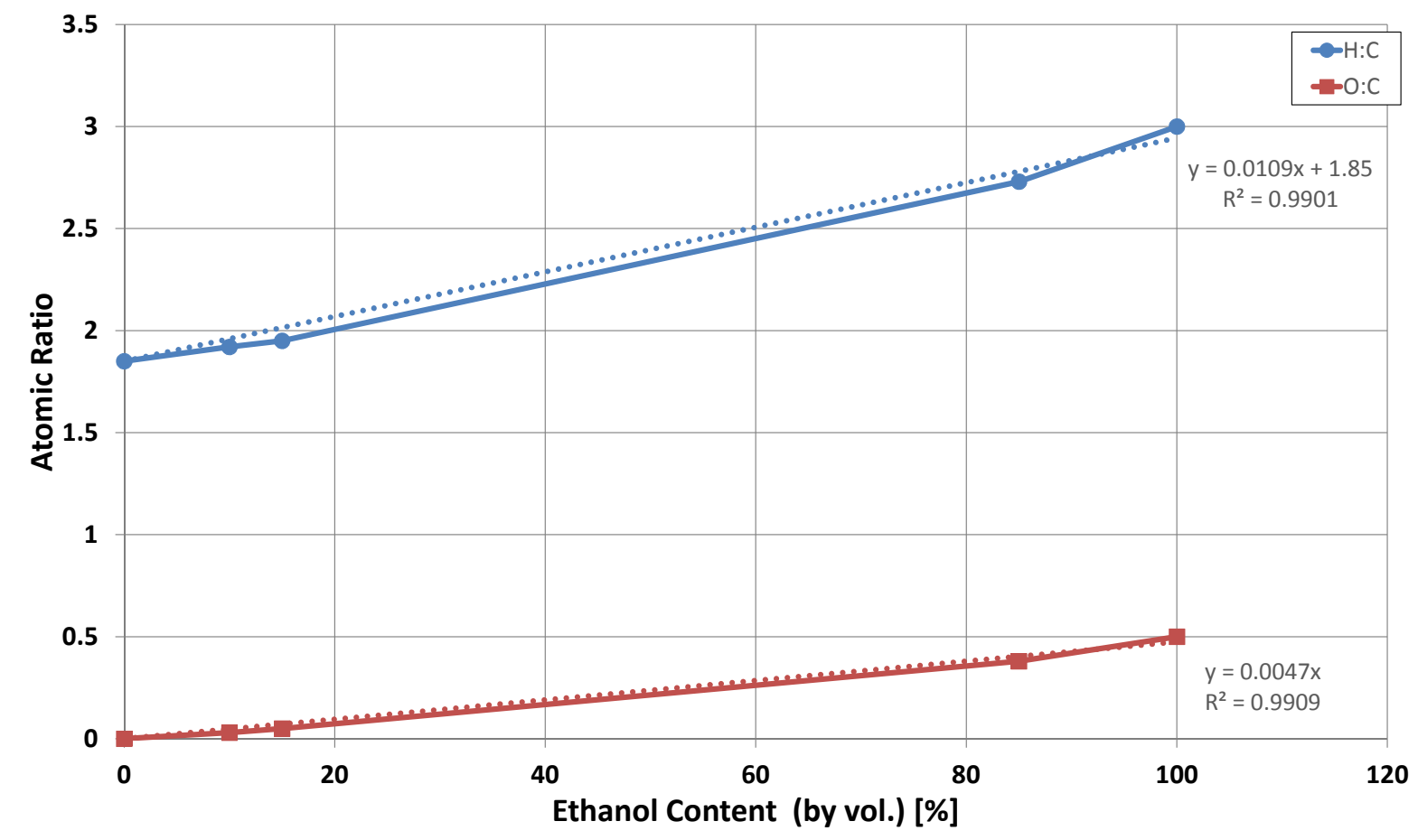

Figure A.1: Atomic ratios regression for increasing ethanol content.

Table A.1: Atomic ratios used for regression curves [79]. Values are normalized to carbon atoms.

\begin{tabular}{cc}
\hline Test Fuel & Atomic Ratio (Normalized) \\
\hline E0 & $\mathrm{CH}_{1.85} \mathrm{O}_{0} \mathrm{~N}_{0}$ \\
E10 & $\mathrm{CH}_{1.92} \mathrm{O}_{0.03} \mathrm{~N}_{0}$ \\
E15 & $\mathrm{CH}_{1.95} \mathrm{O}_{0.05} \mathrm{~N}_{0}$ \\
E85 & $\mathrm{CH}_{2.73} \mathrm{O}_{0.38} \mathrm{~N}_{0}$ \\
E100 & $\mathrm{CH}_{3} \mathrm{O}_{0.5} \mathrm{~N}_{0}$ \\
\hline
\end{tabular}

Using the equations of the regression in Figure A.1, atomic ratios for E30 were calculated to be $\mathrm{CH}_{2.18} \mathrm{O}_{0.141} \mathrm{~N}_{0}$. 


\section{A.2.2 Atomic Ratio Calculations for Toluene Blends}

The following outlines the calculations for the atomic ratios for the toluene blends. These were calculated on a volumetric basis using the values listed in Table A.2.

Table A.2: Chemical constituents and corresponding atomic ratios for the pure fuel components. Values are normalized to carbon atoms.

\begin{tabular}{ccc}
\hline Constituent & Chemical Formula & Atomic Ratio (Normalized) \\
\hline Gasoline (E0) & N/A & $\mathrm{CH}_{1.85} \mathrm{O}_{0} \mathrm{~N}_{0}$ \\
Ethanol & $\mathrm{C}_{2} \mathrm{H}_{5} \mathrm{OH}$ & $\mathrm{CH}_{3} \mathrm{O}_{0.5} \mathrm{~N}_{0}$ \\
Toluene & $\mathrm{C}_{7} \mathrm{H}_{8}$ & $\mathrm{CH}_{1.143} \mathrm{O}_{0} \mathrm{~N}_{0}$ \\
\hline
\end{tabular}

Calculations for the two toluene based fuel blends:

T10 (10\% toluene by vol. and $90 \%$ gasoline by vol.) fuel blend calculation:

$$
\begin{aligned}
T 10 & =0.9(E 0)+0.1(\text { Toluene }) \\
& =0.9\left(\mathrm{CH}_{1.85} \mathrm{O}_{0} \mathrm{~N}_{0}\right)+0.1\left(\mathrm{CH}_{1.143} \mathrm{O}_{0} \mathrm{~N}_{0}\right) \\
& =C H_{1.78} \mathrm{O}_{0} \mathrm{~N}_{0}
\end{aligned}
$$

T10E10 (10\% toluene by vol. - 10\% ethanol by vol. - $80 \%$ gasoline by vol.) fuel blend calculation:

$$
\begin{aligned}
T 10 E 10 & =0.8(E 0)+0.1(\text { Toluene })+0.1(\text { Ethanol }) \\
& =0.8\left(\mathrm{CH}_{1.85} \mathrm{O}_{0} N_{0}\right)+0.1\left(\mathrm{CH}_{1.143} \mathrm{O}_{0} \mathrm{~N}_{0}\right)+0.1\left(\mathrm{CH}_{3} \mathrm{O}_{0.5} N_{0}\right) \\
& =C H_{1.89} \mathrm{O}_{0.03} N_{0}
\end{aligned}
$$

NOTE: $O$ atomic ratio in A.2 is forced to the value for E10 per the US EPA.

\section{A.3 Dry Air Supply}

Table A.3 contains the specifications on the dry air supply used for the two diluters (Dekati and Rotating Disk Thermodiluter) and the emissions bench presented in Chapter 4. The order of filters presented in Table A.3 indicate the order in the physical arrangement.

Table A.3: Details of the dry air supply used by the diluters in this study.

\begin{tabular}{llcc}
\hline Filter Type & Filter Part No. & Filtration/Dew Point & Max. Flow Rate (scfm) \\
\hline Coarse Air Filter & Norgren F73G-4AN-QD1 & $5 \mu \mathrm{m}$ & 62 \\
Desiccant Air Drier & Parker DD30-04 & $-40{ }^{\circ} \mathrm{C}$ & 30 \\
Fine Air Filter (1) & Motor Guard Corp M60 & $0.01 \mu \mathrm{m}$ & 110 \\
Fine Air Filter (2) & SMC AWD40-N04DE-Z & $0.01 \mu \mathrm{m}$ & 17 \\
\hline
\end{tabular}




\section{Appendix B}

\section{Chapter 5 Calculations}

\section{B.1 Road Load Calculations}

The following details the road load calculation for determining the engine speed and load set-point at a typical highway cruise vehicle speed. Vehicle specifications are contained in Table B.1. Equation B.2 is from Heywood [8]. Note that Equation B.2 does not show the appropriate unit conversion necessary for the final answers presented.

Table B.1: Road load engine and vehicle parameters.

\begin{tabular}{lcc}
\hline Parameter & Variable in Equation & Value \\
\hline Vehicle Speed $(\mathrm{km} / \mathrm{h})$ & $S_{v}$ & 100 \\
Tire Revolutions per km $(\mathrm{rev} / \mathrm{km})[89,98]$ & $\mathrm{TR}$ & 512 \\
Top Gear Ratio [89] & $\mathrm{TG}$ & 0.81 \\
Final Drive Ratio [89] & $\mathrm{FG}$ & 3.82 \\
\hline Curb Weight $(\mathrm{kg})[89]$ & $M_{v}$ & 1392 \\
Vehicle Frontal Area $\left(\mathrm{m}^{2}\right)[89]$ & $A_{v}$ & 2.673 \\
Aerodynamic Drag Coefficient & $C_{D}$ & 0.32 \\
Rolling Resistance Coefficient $[8,98]$ & $C_{R}$ & 0.0135 \\
\hline
\end{tabular}

Calculated Engine Speed at $100 \mathrm{~km} / \mathrm{h}$ :

$$
\begin{aligned}
\text { EngineSpeed } & =\frac{T G \times F G \times T R \times S_{v}}{60 \mathrm{~min} / \mathrm{h}} \\
& =2640 \mathrm{rpm}
\end{aligned}
$$

Calculated Engine Speed at $100 \mathrm{~km} / \mathrm{h}$ :

$$
\begin{aligned}
P_{r} & =\left(\frac{1}{2} \rho_{a i r} \times C_{D} \times A_{v} \times S_{v}^{2}+C_{R} \times M_{v} \times g\right) \times S_{v} \\
& =16.1 \mathrm{~kW}=42.7 \mathrm{ft}-\mathrm{lb}
\end{aligned}
$$




\section{B.2 Emissions Bench Oxygen Correction}

The following details the procedure for the oxygen correction. To find the corrected oxygen requires the use of measured species concentrations from the emissions bench, along with some assumed values. Equations B.3 and B.4 listed below are from Heywood [8].

\section{B.2.1 Oxygen Correction Calculation}

\section{Nomenclature:}

$\tilde{x}_{i}^{*}$ - Species dry mole fraction

$\tilde{x}_{i}$ - Species wet mole fraction

Find calculated exhaust water content:

$$
\tilde{x}_{\mathrm{H}_{2} \mathrm{O}}=\frac{m}{2 n}\left[\frac{\tilde{x}_{\mathrm{CO}}^{*}+\tilde{x}_{\mathrm{CO}}^{*}}{1+\tilde{x}_{\mathrm{CO}}^{*} /\left(K \tilde{x}_{C O_{2}}^{*}\right)+(m / 2 n)\left(\tilde{x}_{C O}^{*}+\tilde{x}_{C O_{2}}^{*}\right)}\right]
$$

where:

$\tilde{x}_{\mathrm{H}_{2} \mathrm{O}}$ - calculated water mole fraction

$\tilde{x}_{C O}^{*}$ - dry $\mathrm{CO}$ mole fraction from the emissions bench

$\tilde{x}_{C O}^{*}$ - dry $\mathrm{CO}_{2}$ mole fraction from the emissions bench

$m \& n$ - carbon and hydrogen atoms in the fuel, of form $C_{n} H_{m}$

$K$ - empirical constant assumed here to be 3.8, per Heywood [8]

Calculate dry $\mathrm{NO}_{\mathrm{x}}$ :

$$
\tilde{x}_{N O_{\mathrm{x}}}^{*}=\frac{\tilde{x}_{N O_{\mathrm{x}}}}{1-\tilde{x}_{\mathrm{H}_{2} \mathrm{O}}}
$$

where:

$\tilde{x}_{C O}$ - wet $\mathrm{NO}_{\mathrm{x}}$ mole fraction from the emissions bench

$\tilde{x}_{\mathrm{H}_{2} \mathrm{O}}$ - water mole fraction as calculated in B.3

Corrected $\mathrm{O}_{2}$ concentration: Correction values are taken from Figure B. 1 at $50{ }^{\circ} \mathrm{C}$. Equation B.5 is from correction procedure provided by CAI [87].

$$
\bar{x}_{O_{2}}^{*}=\tilde{x}_{O_{2}}^{*}-\left(-0.29 / 100\left(\tilde{x}_{C O_{2}}^{*}\right)-0.07 / 100\left(\tilde{x}_{C O}^{*}\right)+43 / 100\left(\tilde{x}_{N O_{\mathbf{x}}}^{*}\right)\right)
$$

where:

$\bar{x}_{\mathrm{O}_{2}}^{*}$ - corrected dry mole fraction of $\mathrm{O}_{2}$

$\tilde{x}_{\mathrm{O}_{2}}^{*}$ - dry $\mathrm{O}_{2}$ mole fraction from emissions bench

$\tilde{x}_{\mathrm{CO}_{2}}^{*}$ - dry $\mathrm{CO}_{2}$ mole fraction from emissions bench

$\tilde{x}_{C O}^{*}$ - dry $\mathrm{CO}$ mole fraction from emissions bench

$\tilde{x}_{N O_{\mathrm{x}}}^{*}-$ dry $\mathrm{NO}_{\mathrm{x}}$ mole fraction from equation B.4 


\section{B.2.2 Oxygen Correction Factors}

Section 11

Model 600P - Paramagnetic Oxygen Analyzer

Table 4-1 Cross Sensitivity of gases

All values based on nitrogen $0 \%$ / oxygen $100 \%$

\begin{tabular}{|c|c|c|c|}
\hline Gas & Formula & $20^{\circ} \mathrm{C}$ & $50^{\circ} \mathrm{C}$ \\
\hline Argon & $\mathrm{Ar}$ & -0.23 & -0.25 \\
\hline Acetylene & $\mathrm{C}_{2} \mathrm{H}_{2}$ & -0.26 & -0.28 \\
\hline Acetone & $\mathrm{C}_{3} \mathrm{H}_{6} \mathrm{O}$ & -0.63 & -0.69 \\
\hline Acetaidehyde & $\mathrm{C}_{2} \mathrm{H}_{4} \mathrm{O}$ & -0.31 & -0.34 \\
\hline Ammonia & $\mathrm{N}_{3}$ & -0.17 & -0.19 \\
\hline Benzene & $\mathrm{C}_{6} \mathrm{H}_{4}$ & -1.24 & -1.34 \\
\hline Bromine & $\mathrm{Br}_{2}$ & -1.78 & -1.97 \\
\hline Butadiene & $\mathrm{C}_{4} \mathrm{H}_{6}$ & -0.85 & -0.93 \\
\hline Isobutylene & $\left(\mathrm{CH}_{3}\right) 2 \mathrm{CH}=\mathrm{CH}_{2}$ & -0.94 & -1.06 \\
\hline n-Butane & $\mathrm{C}_{4} \mathrm{H}_{10}$ & -1.10 & -1.22 \\
\hline Chlorine & $\mathrm{CL}_{2}$ & -0.83 & -0.91 \\
\hline Hydrogen Chloride & $\mathrm{HCL}$ & -0.31 & -0.34 \\
\hline Nitrous Oxide & $\mathrm{N}_{2} \mathrm{O}$ & -0.20 & -0.22 \\
\hline Diacetylene & $(\mathrm{CHCl})_{2}$ & -1.09 & -1.20 \\
\hline Ethane & $\mathrm{C}_{2} \mathrm{H}_{4}$ & -0.43 & -0.47 \\
\hline Ethylene Oxide & $\mathrm{C}_{2} \mathrm{H}_{4} \mathrm{O}_{2}$ & -0.54 & -0.60 \\
\hline Ethylene & $\mathrm{C}_{2} \mathrm{H}_{4}$ & -0.20 & -0.22 \\
\hline Ethylene Glycol & $\mathrm{CH}_{2} \mathrm{OHCH}_{2} \mathrm{OH}$ & -0.78 & -0.88 \\
\hline Ethylbenzene & $\mathrm{C}_{8} \mathrm{H}_{10}$ & -1.89 & -2.08 \\
\hline Hydrogen Fluoride & $\mathrm{HF}$ & +0.12 & +0.14 \\
\hline Furan & $\mathrm{C}_{4} \mathrm{H}_{4} \mathrm{O}$ & -0.90 & -0.99 \\
\hline Helium & $\mathrm{He}$ & +0.29 & +0.32 \\
\hline n-Hexane & $\mathrm{C}_{6} \mathrm{H}_{14}$ & -1.78 & -1.97 \\
\hline Krypton & $\mathrm{Kr}$ & -0.49 & -0.54 \\
\hline Carbon Monoxide & $\mathrm{CO}$ & -0.06 & -0.07 \\
\hline Carbon Dioxide & $\mathrm{CO}_{2}$ & -0.27 & -0.29 \\
\hline Methane & $\mathrm{CH}_{4}$ & -0.16 & -0.17 \\
\hline Methanol & $\mathrm{CH}_{4} \mathrm{O}$ & -0.27 & -0.31 \\
\hline Methylene Chloride & $\mathrm{CH}_{2} \mathrm{Cl}_{2}$ & -1.00 & -1.10 \\
\hline Neon & $\mathrm{Ne}$ & +0.16 & +0.17 \\
\hline n-Octane & $\mathrm{C}_{8} \mathrm{H}_{18}$ & -2.45 & -2.70 \\
\hline Phenol & $\mathrm{C}_{6} \mathrm{H}_{6} \mathrm{O}$ & -1.40 & -1.54 \\
\hline Propane & $\mathrm{C}_{3} \mathrm{H}_{8}$ & -0.77 & -0.85 \\
\hline Propylene & $\mathrm{C}_{3} \mathrm{H}_{6}$ & -0.57 & -0.62 \\
\hline Propene & $\mathrm{CH}_{3} \mathrm{CH}=\mathrm{CH}_{12}$ & -0.58 & -0.64 \\
\hline Propylene Oxide & $\mathrm{C}_{3} \mathrm{H}_{6} \mathrm{O}$ & -0.90 & -1.00 \\
\hline Propylene Chloride & $\mathrm{C}_{3} \mathrm{H}_{7} \mathrm{Cl}$ & -1.42 & -1.44 \\
\hline Silane & $\mathrm{SiH}_{4}$ & -0.24 & -0.27 \\
\hline Styrene & $\mathrm{C}_{7} \mathrm{H}_{6}=\mathrm{CH}_{2}$ & -1.63 & -1.80 \\
\hline Nitrogen & $\mathrm{N}_{2}$ & -0.00 & -0.00 \\
\hline Nitrogen Monoxide & NO & +42.70 & +43.00 \\
\hline Nitrogen Dioxide & $\mathrm{NO}_{2}$ & +5.00 & +16.00 \\
\hline Oxygen & $\mathrm{O}_{2}$ & +100.00 & +100.00 \\
\hline Sulfur Dioxide & $\mathrm{SO}_{2}$ & -0.18 & -0.20 \\
\hline Sulfur Fluoride & $\mathrm{SF}_{6}$ & -0.98 & -1.05 \\
\hline Hydrogen Sulfide & $\mathrm{H}_{2} \mathrm{~S}$ & -0.41 & -0.43 \\
\hline Toluene & $\mathrm{C}_{7} \mathrm{H}_{8}$ & -1.57 & -1.73 \\
\hline Trichloroethylene & $\mathrm{C}_{2} \mathrm{HCl}_{3}$ & -1.56 & -1.72 \\
\hline Vinyl Chloride & $\mathrm{C}_{2} \mathrm{H}_{3} \mathrm{Cl}$ & -0.68 & -0.74 \\
\hline Vinyl.Fluoride & $\mathrm{CH}_{3} \mathrm{~F}$ & -0.49 & -0.54 \\
\hline Water & $\mathrm{H}_{2} \mathrm{O}$ & -0.03 & -0.03 \\
\hline Hydrogen & $\mathrm{H}_{2}$ & +0.23 & +0.26 \\
\hline Xenon & $\mathrm{Xe}$ & -0.95 & -1.02 \\
\hline
\end{tabular}

California Analytical Model 600P Rev 12 C_ETL_US_CE Operators Manual

Page 63 of 96

Figure B.1: Oxygen measurement correction factors [87]. 


\section{B.3 Dilution Ratio Calculation}

The following provides a derivation of the dilution ratio calculation, which was used in this investigation to calculate true dilution ratios from the two diluters. The calculation is based on raw exhaust $\mathrm{CO}_{2}$ (upstream) concentrations from the emissions bench and diluted exhaust $\mathrm{CO}_{2}$ (downstream) concentrations from the Li-Cor mini $\mathrm{CO}_{2}$ monitor.

Conservation of Mass: assume a control volume around diluter, with raw exhaust and dilution air coming in, and diluted exhaust coming out.

$$
\dot{m}_{\text {exhaust }_{\text {diluted }}}=\dot{m}_{\text {exhaust }_{\text {raw }}}+\dot{m}_{\text {dilution air }}
$$

Conservation of Species: assume no $\mathrm{CO}_{2}$ is destroyed or generated during dilution.

$$
\dot{m}_{\mathrm{CO}_{2} \text { exhaust }_{\text {diluted }}}=\dot{m}_{\mathrm{CO}_{2} \text { exhaust }_{\text {raw }}}+\dot{m}_{\mathrm{CO} 2 \text { dilution air }}
$$

where:

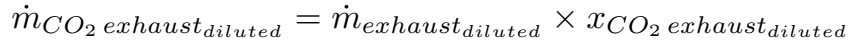

$$
\begin{aligned}
& \dot{m}_{\mathrm{CO}_{2} \text { exhaust }_{\text {raw }}}=\dot{m}_{\text {exhaust }_{\text {raw }}} \times x_{\mathrm{CO}_{2}} \text { exhaust }_{\text {diluted }} \\
& \dot{m}_{C O 2 \text { dilution air }}=\dot{m}_{\text {dilution air }} \times x_{C O 2 \text { dilution air }}
\end{aligned}
$$

\section{Combination of equations and re-arranging:}

Combine Equation B.7 with Equations (a), (b), and (c):

$\dot{m}_{\text {exhaust }_{\text {diluted }}} \times x_{\mathrm{CO}_{2} \text { exhaust }_{\text {diluted }}}=\dot{m}_{\text {exhaust }_{\text {raw }}} \times x_{\mathrm{CO}_{2} \text { exhaust }_{\text {diluted }}}+\dot{m}_{\text {dilution air }} \times x_{C O 2 \text { dilution air }}$

Rearranging Equation B.8:

$$
x_{\mathrm{CO}_{2} \text { exhaust }_{\text {diluted }}}=\frac{\dot{m}_{\text {exhaust }_{\text {raw }}} \times x_{\mathrm{CO}_{2} \text { exhaust }_{\text {diluted }}}+\dot{m}_{\text {dilution air }} \times x_{\mathrm{CO} 2 \text { dilution air }}}{\dot{m}_{\text {exhaust }} \text { diluted }_{\text {ilu }}}
$$

Sub in Equation B.6 into Equation B.9:

$x_{\mathrm{CO}_{2} \text { exhaust }_{\text {diluted }}}=\frac{\dot{m}_{\text {exhaust }_{\text {raw }}} \times x_{\mathrm{CO}_{2} \text { exhaust }_{\text {raw }}}+\left(\dot{m}_{\text {exhaust }_{\text {diluted }}}-\dot{m}_{\text {exhaust }_{\text {raw }}}\right) \times x_{\mathrm{CO}_{2} \text { dilution air }_{\text {air }}}}{\dot{m}_{\text {exhaust }} \text { diluted }}$

Multiply out Equation B.10 and collect like terms:

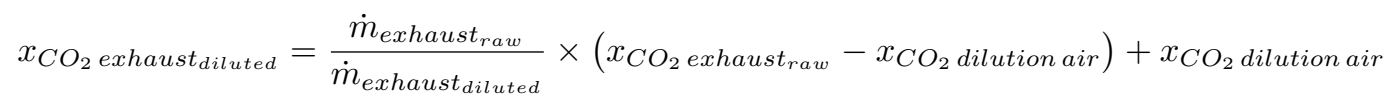

Solve for dilution ratio:

$$
\text { Dilution Ratio }=\frac{\dot{m}_{\text {exhaust }_{\text {raw }}}}{\dot{m}_{\text {exhaust }} \text { diluted }}=\frac{x_{\mathrm{CO}_{2} \text { exhaust }_{\text {raw }}}-x_{\mathrm{CO}_{2} \text { dilution air }}}{x_{\mathrm{CO}_{2} \text { exhaust }_{\text {diluted }}}-x_{\mathrm{CO}_{2} \text { dilution air }}}
$$




\section{Appendix C}

\section{Chapter 6 Supplementary Notes, Discussions, and Calculations}

\section{C.1 Engine Cleaning Procedure}

A commercially available solvent designed for engine deposit removal was initially sourced to clean the engine. The solvent is primarily comprised of petroleum products (pale oil, naphtha, isopropyl alcohol) and is designed to clean induction (air intake), fuel system, and combustion chamber deposits [99]. For the cleaning performed here, an appropriate amount was added to the fuel to also clean the fuel system and injectors of any built up deposits. Solvent was likewise added to the crankcase via the oil filler port to clean any crankcase deposits formed; a required oil change was performed after the cleaning. The procedure to clean the intake system involves bringing the engine up to operating temperature, followed by inducing the solvent into the intake manifold using the vacuum generated while in operation. The solvent is then allowed to soak for a 20 minute period on all the wetted surfaces with the engine off. The engine is then restarted and the run relatively hard (higher speed and load) to burn off the carbonaceous deposits.

With the above method proving to insufficiently remove the deposits, a second cleaning method was performed. With the engine removed from commission, a thorough mechanical cleaning of the induction system was performed. A generic carburetor cleaner, also a cocktail of petroleum products (xylene, ethylbenzene, acetone, propane, isobutene, and diacetone alcohol) [100], was used to soak the deposits while wire brushes and metal picks provided mechanical agitation and removal. This process was repeated several times until the intake valves and runners were deemed clean. Additionally, the intake manifold was cleaned with dish soap to remove the built up oil coating its inside, while the carburettor cleaner was also used to clean the throttle body.

\section{C.2 Fuel Trim Drift: Marker for Temporal Variations}

One of the OBDII PIDs available on this research engine - the short-term fuel trim (STFT) - was identified as being potentially relevant to the discussion of fuel composition and PM emissions. The PCM equipped on this engine, and those found on production vehicles, have fuel trimming authority granted to them to appropriately adjust the fuel mass delivered for current conditions. Using the base engine 
map as its reference point, the PCM can trim the fuel up (enrich) or down (lean-out) based on feedback from the equipped oxygen sensor(s) [101]. Two trimming parameters are available, those being the STFT and the LTFT. As their names suggest, the STFT updates fuel delivery frequently, while the LTFT will be modified if STFT values remain consistently high or low over a longer time period [101]. Returning to the data in question, comparing the STFT values of the different fuel blends showed that differing amounts of fuel trimming occurred depending on the fuel being used. Based on this information, further scrutiny of the data was performed, specifically looking at whether the STFT was trending with the emissions increase with test runtime. The plot in Figure C.1 presents test group averaged PN concentration data normalised for maximum emission. This data presentation allows for better comparison of PN concentration behaviour without dealing with large differences in magnitude, as shown in Figure 6.13. A dashed line labelled "Drift Cut-Off" is a point of reference used here to denote when the PM emissions had reached near steady-state values. This selection is largely arbitrary, though inspecting the plotted data shows that all of the fuel blends experienced slope reductions at or near this point, indicating that steady-state emission was achieved or nearly achieved. Inspection of this figure shows that of all the fuel blends tested, both E0 data sets (E0 and E0 Return) reached 90\% of maximum emission the fastest. Increasing either the toluene or ethanol fraction of the fuel resulted in delaying achievement of this threshold, with E30 requiring 60 minutes on average. These results are laid out plainly here, but have already been alluded to in previous discussions. Comparing the data presented in Figure C.2 paints a different picture altogether. This figure contains the normalised STFT values with test run time averaged for each test group; a similar plot to the PN plot presented above. Using the same cut-off point of $90 \%$ shows a complete trend reversal, with the E30 reaching $90 \%$ of max STFT the soonest. Decreasing the ethanol content required longer times to reach this threshold, with the pure gasoline (E0 and E0 Return) requiring between 40 and 50 minutes to reach this value. Based on this observation, it seems that the PN emissions do not track well with the changes in STFT. Therefore, even though a similar drifting behaviour was noted for STFT, it is likely not the source of the temporal variation in PM emissions seen in this work.

Before leaving the topic of fuel trims, it should be noted that another possible explanation could be useful here. Modern PCMs have the capability to adapt their strategies based on values stored in memory [101] - the LTFT would be one such parameter. Returning to the STFT behaviour, each and every test performed appears to have started from the same base map values. While it is expected that during starting the PCM reverts to its pre-programmed start map [101], changes to the LTFT would be expected after several runs if the STFT climbed to high enough values. As an example, each E30 started with STFT values near 0\% and climbed up to over $13 \%$ before test end. Over three runs, it would be expected that adjustments to the LTFT would be made to facilitate the appropriate trimming of the fuel for this blend. However, based on the behaviour noted this was not the case. Looking at the engine set-up, it was determined that typical shut-down protocol requiring the removal of terminal leads from the automotive battery would prevent the storage of these values. Removing the terminal leads on a production vehicle essentially erases the memory of the PCM, including any fault codes. Several tests were performed to try and keep the PCM energized, thereby retaining the "learned" STFT values in memory with the hopes that adjustments would be made to the LTFT values. Unfortunately, no changes in behaviour were observed and the problem was traced back to the dynamometer harness received with the engine. It is wired in such a way that cutting the ignition - required to shut-off the engine - actually cuts the power to the PCM entirely. Therefore, each time the engine was shut off, any learning that the 


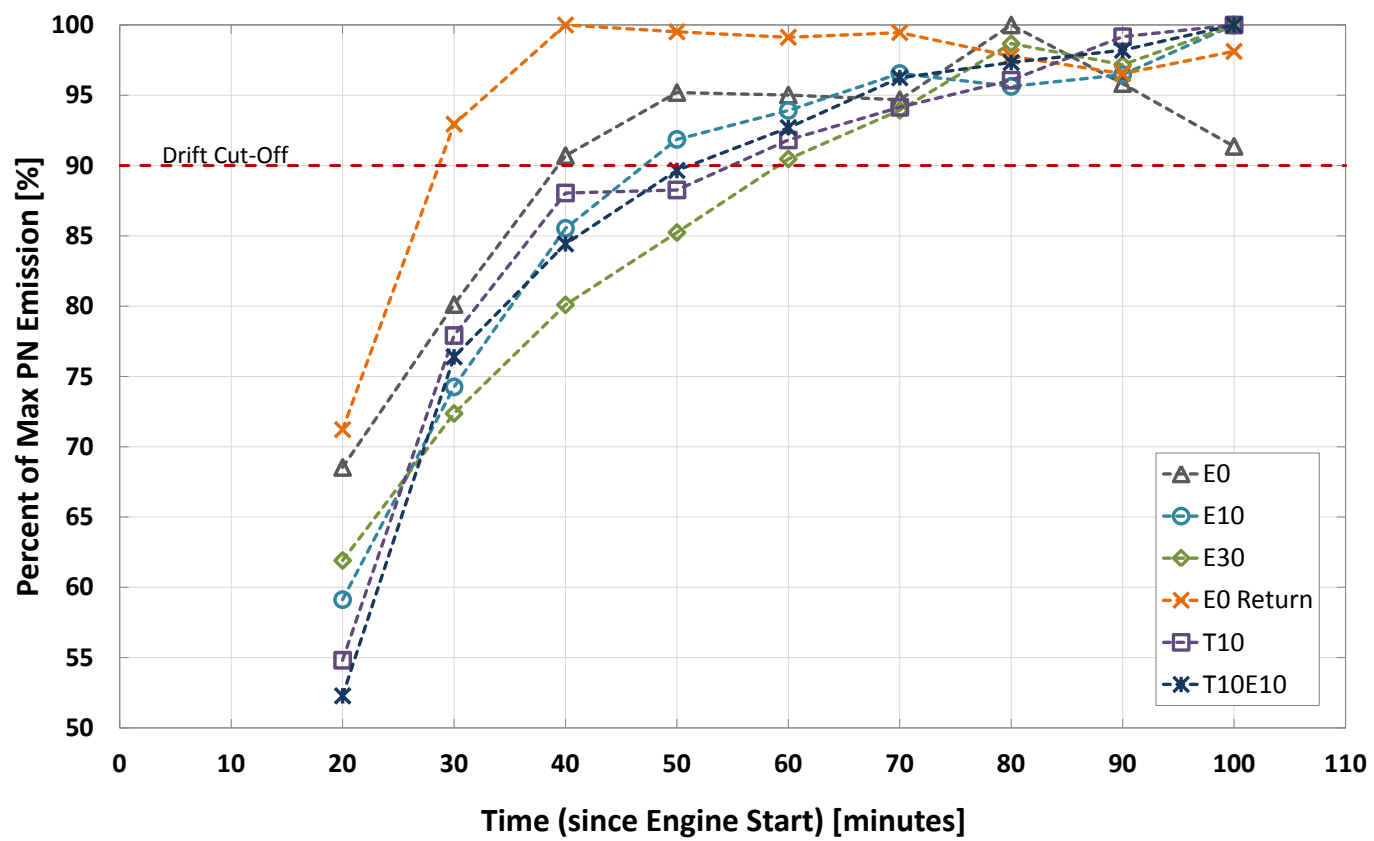

Figure C.1: Percentage of maximum PN concentration with run time, averaged for each test group.

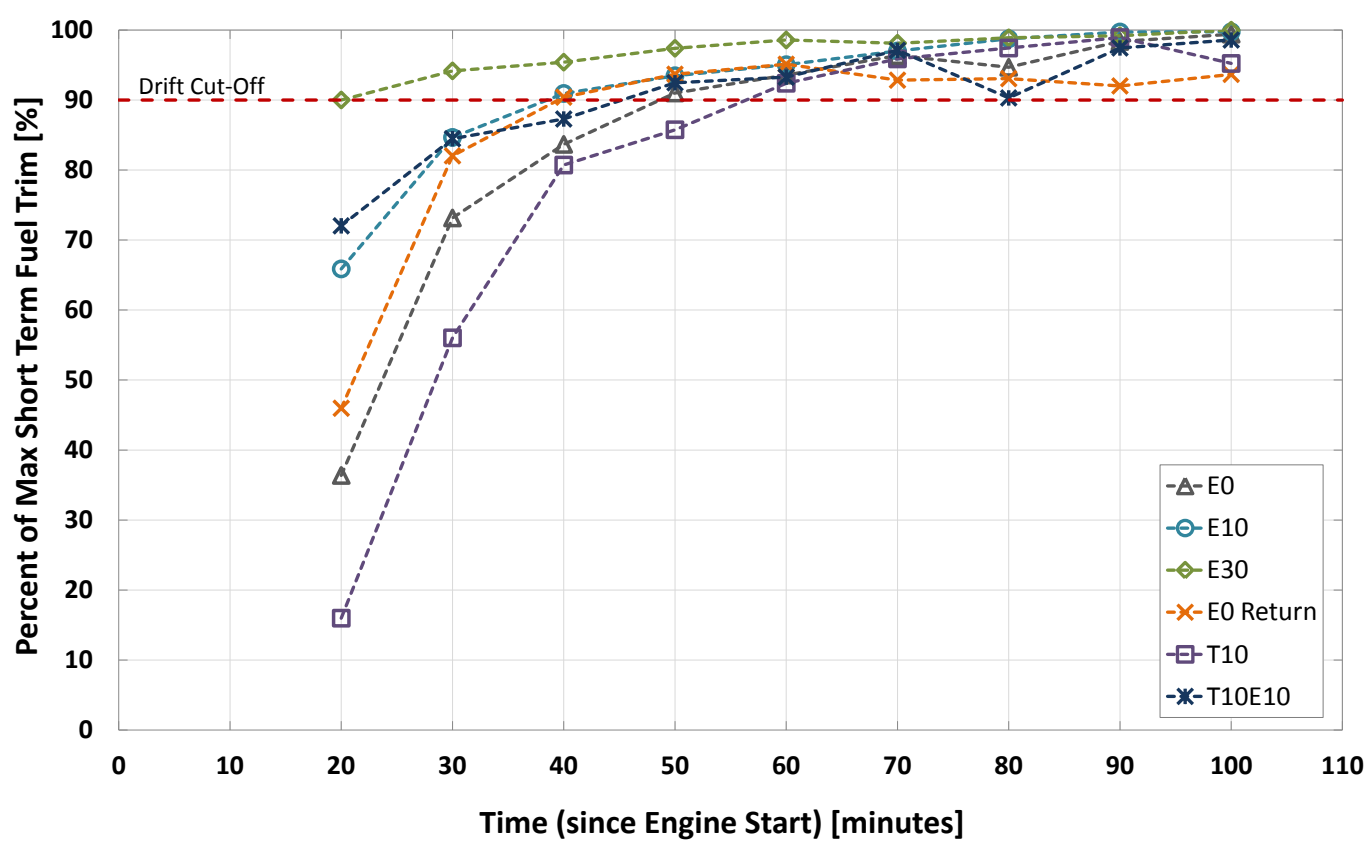

Figure C.2: Percentage of maximum short-term fuel trim with run time, averaged for each test group.

ECU had potentially done was effectively erased. It is important to consider that perhaps this function was already disabled with this PCM, as it was previously used for durability testing purposes, therefore rendering this argument moot. Therefore, even if the issue of cutting power to the PCM were fixed, the PCM may not currently support adaptive learning. While the presented discussion has put the notion of PM emissions trending with STFT to rest, there still remains the question of whether the observed variability is PCM related. Further data should be collected to verify this, and to determine whether 
this PCM is suitable for the future work on this engine.

\section{C.3 Exhaust Temperature: Marker for Combustion Stability}

One of the presented observations with regards to fuel composition was the increasing variability with increasing ethanol and toluene fractions in the fuel blend. One hypothesis is that this increased emissions variability was caused by a decrease in combustion stability, or in other words an increase in combustion variability. Indeed, Wallner and Miers [32] found a fuel dependency on combustion stability, with greater proportions of ethanol causing decreased stability, though not sufficiently to reduce driveability. A similar result was also observed in the work of Chen et al. [22], even at fully warmed conditions. A commonly used metric of combustion variability is the measurement of cyclic variability from in-cylinder pressure data given by the coefficient of variation in indicated mean effective pressure [8]. Driveability issues can arise if this $\mathrm{CoV}$ exceeds $10 \%$ [8]. In-cylinder pressure measurements required to calculate CoV of IMEP values were not available on the current set-up. However, another indicator that can be used to measure combustion variability is exhaust temperature as an indicator for slow burning. CoV of IMEP values tend to increase as the combustion phasing (time of maximum pressure) is retarded with respect to TDC [8]. A by-product of slow burning combustion is an increase in exhaust temperature due to the decrease in available time for expansion; i.e. the combustion products are expanded less because they burned later into the expansion stroke. In fact, cold idle operation typically uses retarded spark timings for this very reason, to increase exhaust temperatures to warm up the engine and emissions control equipment. Therefore, the exhaust temperature was selected here for further scrutiny; measurements made at the exhaust manifold ( T/C 1) were used in this analysis due to it being the closest exhaust temperature measurement to the engine.

Specific exhaust temperatures averaged for each of the fuel blends tested are listed in Table C.1. The specific exhaust temperature is calculated from the average measured exhaust temperature at fully warm, highway cruise operation and the corresponding average measured engine load. Normalising the exhaust temperature to the engine load removes the influence of engine loading changes on the measured exhaust temperature. Using the calculated confidence interval for the averages shows that E10 and E30 fuelled tests produced lower exhaust temperature when compared to the E0 test group. The toluene blended fuels did not result in a significant difference in exhaust temperature over pure gasoline. These results seemingly contradict the proposed hypothesis that the higher ethanol blends resulted in greater $\mathrm{CoV}$ of IMEP, indicated here by exhaust temperature. However, this result is altogether not unexpected. First, ethanol has a greater laminar flame speed than typical gasoline ( $\sim 39$ vs. $\sim 33 \mathrm{~cm} / \mathrm{s}[32])$, so having lower exhaust temperatures might actually indicate that combustion phasing was advanced as, one might expect given this information. Furthermore, the high latent heat of vaporization of ethanol [32] provides for greater charge cooling leading to reduced peak pressures and temperatures at constant spark timing. In the case of this investigation, the spark timing advance was typically $45^{\circ} \mathrm{CAD}$ BTDC of compression, and did not vary regardless of the fuel. Therefore, a reduction in measured exhaust temperature is not surprising. Given this information one can say that combustion instability was likely a non factor in this investigation. 
Table C.1: Average specific exhaust temperature and corresponding 95\% confidence intervals for each fuel blend.

\begin{tabular}{ccc}
\hline Fuel Blend & Specific Exhaust Temperature $\left({ }^{\circ} \mathrm{C} / \mathrm{ft}-\mathrm{lb}\right)$ & $\pm 95 \% \mathrm{CI}$ \\
\hline E0 & 15.69 & 0.12 \\
E10 & 15.29 & 0.15 \\
E30 & 15.33 & 0.19 \\
E0 Return & 15.73 & 0.47 \\
T10 & 15.59 & 0.45 \\
T10E10 & 15.72 & 0.20 \\
\hline
\end{tabular}

\section{C.4 Engine Oil Dilution: Marker for Wall Impingement}

Another engine related hypothesis considered here for the observed increase in PM emissions with increasing ethanol, is the possibility that an increased amount of fuel impingement was occurring. As previously mentioned, the latent heat of vaporization of ethanol is higher than typical gasoline formulas, reaching as high as 2-3 times higher on a mass basis at SATP $[32,102]$. This has the benefit of providing charge cooling and allowing for higher compression ratios to be used [32, 28, 23, 22]. However, it has the negative effect of making it more difficult to vaporize the fuel, especially at cold conditions. Optical studies $[22,19]$ showed reduced spray dispersion with increasing ethanol, indicating reduced fuel-air mixing and potentially further spray penetration when compared to pure gasoline. Therefore, it is possible that the higher oxygenate blends studied here were resulting in increased cylinder wall wetting and perhaps introducing the lubricating oil into the combustion process, both of which are sources of PM emission. As measuring the impingement levels were not possible on the current apparatus - some studies have measured heat-fluxes on cylinder surfaces with embedded sensors [19, 103] — another marker for impingement was necessary to verify the validity of this hypothesis. Analysing the lubricating oil for ethanol content could perhaps provide some evidence to a first approximation of the presence of cylinder wall impingement. Having run both oxygenate blends (E10 and E30), an oil sample was analyzed for ethanol content to provide a bearing on the amount of oil dilution, which has been discussed in an earlier section as being related to cylinder wall impingement (see Section 3.2.4). Preliminary oil sample analysis was performed off-site using an FTIR and a water extraction technique, which would provide resolution down to percent levels. This level of resolution was believed to be sufficient given the results in similar studies of fuel diltuion $[58,59,57]$.

Unfortunately, this analysis was unable to detect ethanol in the oil sample and further work, such as gas chromatography-mass spectrometry (GC-MS), was not performed due to scheduling conflicts with the analyst group. This finding in itself does not immediately negate the presence of increasing cylinder wall impingement with ethanol content. One problem is that given the duration spent at highway cruise operation (i.e. fully warmed) it is possible that any ethanol that had made its way into the bulk oil was subsequently boiled off. This is likely a factor here, given that even with the oil cooler enabled oil temperatures stabilized at $98.5{ }^{\circ} \mathrm{C}$ a full $20{ }^{\circ} \mathrm{C}$ above the boiling point of ethanol [103]. Additionally, it could be that dilution levels are too low for the detection level that this analysis provides, so the suitability of this correlation may not be strong enough for the analysis here. A more direct measure of cylinder wall impingement would likely be necessary. 
In summary, with the limited information available, no conclusive comment can be provided here on the presence of increasing cylinder wall impingement with increasing ethanol content. Further work should be pursued in this matter as it could prove useful to explain the apparent increase in PM emissions with increasing ethanol content, and perhaps even provide further avenues for investigation into this ever present variability.

\section{C.5 FTIR vs. Emissions Bench}

A detailed comparison of the FTIR and emissions bench NDIR results is provided here in an attempt to explain the differences noted. Figure C.3 plots the standard emissions from the FTIR and the emissions bench, as well as the FTIR results corrected using the calculated water content from the emissions bench. Comparing the values demonstrates that the FTIR $\mathrm{CO}_{2}$ and $\mathrm{CO}$ concentration measurements were consistently lower than the emissions bench by approximately $7 \%$ and $2 \%$, respectively. The opposite trend was seen for the $\mathrm{NO}_{\mathrm{x}}$, with the FTIR reporting concentrations that were $5 \%$ higher than the emissions bench. Though not presented here, these values were checked for the E0, E10, and E30 test groups and the same conclusion was found, so it is believed this error is independent of fuel composition. A difference in water concentration in Figure C.3 is also evident, where the calculated water concentration using the emissions bench measurements (see Appendix B.2) was slightly higher than the water measured by the FTIR. Therefore, it was hypothesized that this could account for the error. Investigation into this hypothesis was performed by correcting the FTIR concentrations with this calculated water content and comparing them to the initial FTIR results. Inspecting Figure C.3 also shows that this did not account for the error between the compounds; corrected concentrations showed virtually no difference between the FTIR and FTIR (Calculated Water Corr.) data sets.

The above errors noted here can be construed as an indication that the FTIR method is not optimal, and that some compound interference is driving the observed error. However, turning to the literature provides some insight; Wallner and Frazee [34] performed a similar instrument comparison and found similar differences for the above compounds in the $\pm 5 \%$ range. Previous work on this apparatus found these differences to be much higher at approximately 10-15\% depending on the species [44], so there has been some improvement. The thought is that improvements to the ranging of the emissions bench performed prior to this investigation, along with the use of newer calibration gas mixtures, are behind the improved measurement agreement. Furthermore, given the confidence intervals presented in Figure C.3, the differences shown here cannot be said to be significant ( $\mathrm{p}>0.05)$, except for $\mathrm{CO}_{2}$. Therefore, given the information from literature, the current differences appear acceptable for most of the compounds, though more work should be done to root out the error for the $\mathrm{CO}_{2}$ measurements. 


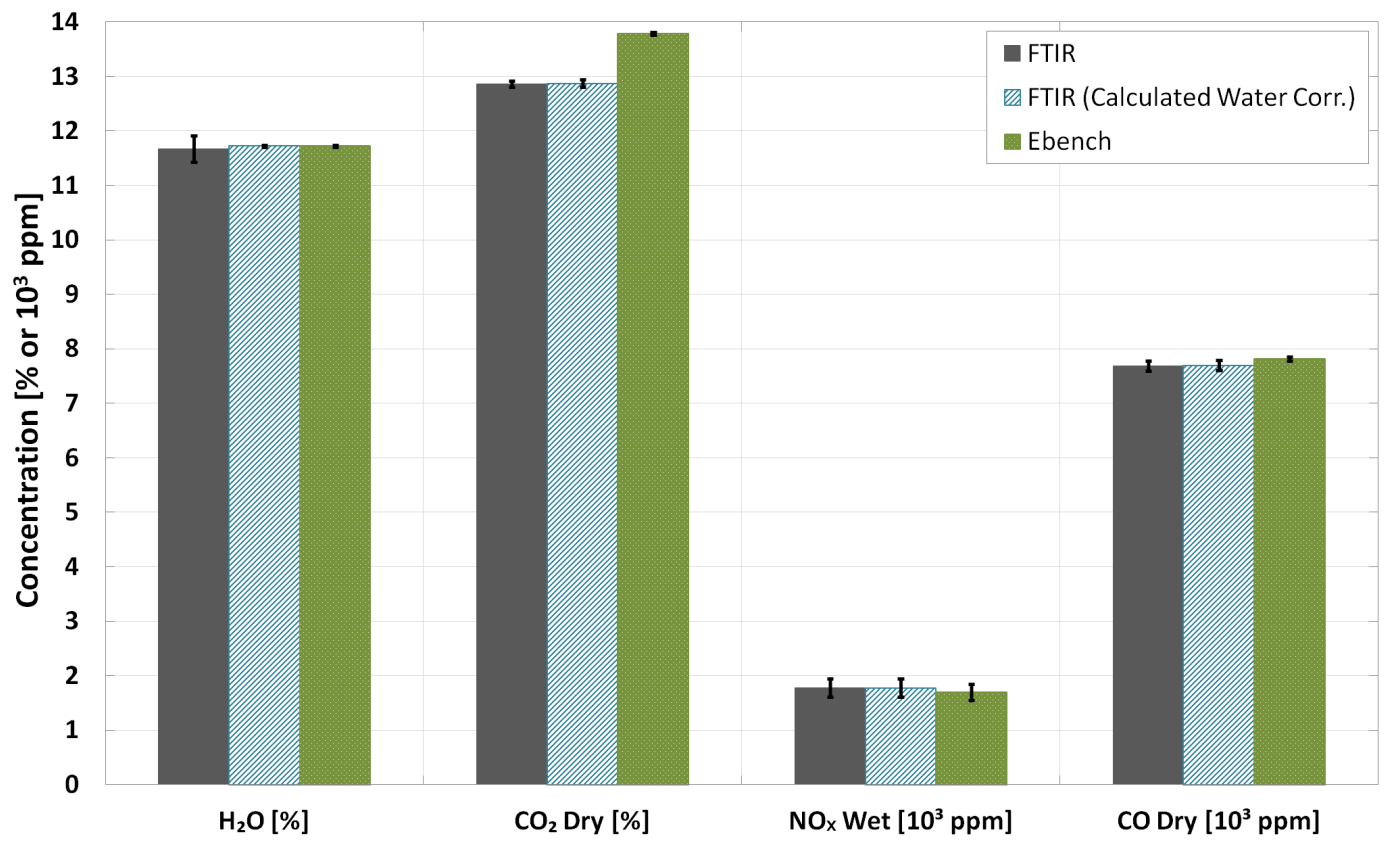

Figure C.3: Comparison of standard emissions measured by the FTIR and the emissions bench. FTIR and Emissions Bench series are the same as in Figures 6.20 and 6.21, respectively. FTIR (Calculated Water Corr.) uses the calculated $\mathrm{H}_{2} \mathrm{O}$ concentration in Figure 6.21 to dry the FTIR emissions. Error bars indicate $95 \%$ confidence intervals. 


\section{C.6 PN Index Calculation}

The following details the calculation of the PN index values presented in Table 6.3 in Section 6.4. The procedure here is largely a simplified version of the procedure presented by Leach et al. [64]. The first simplification arises in the calculation of the vapour pressure of the fuel (DVPE); where Leach et al. used the UNIFAC ${ }^{1}$ method to account for non-ideal mixing, the method here simply uses Raoult's law. The other simplification included using an assumed vapour pressure for the base gasoline, based on the month in which the fuel was obtained. These values were obtained by referencing US EPA standards [96] for the months in question, which are generally applicable to Canada.

\section{C.6.1 Fuel Properties Required for PN Index}

Table C.2 contains the property values for the three constituents used; gasoline (Fuel B), ethanol, and toluene. References for the values are given in the table including to any of the relevant equations below.

Table C.2: Fuel properties for the three fuel constituents used.

\begin{tabular}{|c|c|c|c|c|}
\hline \multirow[b]{2}{*}{ Property } & \multicolumn{3}{|c|}{ Constituent $(i)$} & \multirow[b]{2}{*}{ Source or Equation } \\
\hline & Gasoline & Toluene & Ethanol & \\
\hline Density (kg/L) & 0.77 & 0.867 & 0.785 & {$[8]$} \\
\hline Molecular Weight (kg/kmol) & 105 & 92.14 & 46.07 & {$[8]$} \\
\hline Molecular Formula $(\mathrm{C}, \mathrm{H})$ & $8,14.8$ & 7,8 & 2,6 & {$[8]$ or $[$ C.1] } \\
\hline $\mathrm{DBE}+1$ & 2.6 & 5 & 1 & {$[\mathrm{C} .2]$} \\
\hline Reid Vapour Pressure (psi @ 311 K) & 7.8 & 1.1 & 2.3 & {$[96,104]$} \\
\hline Reid Vapour Pressure (kPa) & 53.78 & 7.58 & 15.86 & $\mathrm{~N} / \mathrm{A}$ \\
\hline
\end{tabular}

\section{Molecular Formula Calculation for Gasoline:}

Assume that the "average" gasoline molecule has 8 carbon atoms and use $\mathrm{H}: \mathrm{C}$ ratio of 1.85:1 per US EPA [79].

$$
\frac{H}{8}=\frac{1.85}{1} \rightarrow H=14.8
$$

Double Bonded Equivalent Calculation:

$$
D B E_{i}=\frac{2 C-H-N+2}{2}
$$

\section{C.6.2 Calculation of PN Index}

The PN index calculation for each fuel can be performed using the above fuel properties. Below is an outline of the general approach to calculate these indices. The final indices, along with calculated DBE +1 scores and DVPE are provided in Table C.3

$\overline{{ }^{1} \text { UNIFAC - UNIversal Functional Activity Coefficient }}$ 


\section{Calculate the mole fraction of each constituent $i$ :}

Find the number of moles in corresponding volume fraction for each constituent assuming total fuel volume is $1 \mathrm{~L}$ for unit conversion.

$$
n_{i}=\frac{V_{i} \times \rho_{i}}{M_{i}}
$$

where:

$$
\begin{aligned}
& V_{i} \text { - volume fraction of constituent } i \text { based on fuel proportions in Section } 4.7 \text { for fuel blend } \\
& \rho_{i} \text { - density of constituent } i \text { from Table C.2 } \\
& M_{i} \text { - molar mass of constituent } i \text { from Table C.2 }
\end{aligned}
$$

Find the mole fractions for each constituent.

$$
x_{i}=\frac{n_{i}}{\sum_{i=1}^{n} n_{i}}
$$

Calculate the DVPE using Raoult's Law:

$$
D V P E=\sum_{i=1}^{n}\left(R V P_{i} \times x_{i}\right)
$$

where:

$R V P_{i}$ - Reid Vapour Pressure of constituent $i$ from Table C.2

$x_{i}$ - mole fraction of constituent $i$ from Equation C.4

$M_{i}$ - molar mass of constituent $i$ from Table C.2

Calculate the PN index using Equation 3.2 presented in Chapter 3:

$$
P N I n d e x=\frac{\sum_{i=1}^{n}\left(D B E_{i}+1\right) V_{i}}{D V P E} \times 100 k P a
$$

Calculate $\mathrm{DBE}+1$ value for the fuel blend

$$
D B E+1_{i}=\sum_{i=1}^{n}\left(D B E_{i}+1\right) V_{i}
$$

Table C.3: Calculated PN indices.

\begin{tabular}{cccc}
\hline Test Fuel & DBE+1 & DVPE $(\mathrm{kPa})$ & PN Index \\
\hline E0 & 2.6 & 53.78 & 4.83 \\
E10 & 2.44 & 46.0 & 5.31 \\
E30 & 2.12 & 34.8 & 6.09 \\
T10 & 2.84 & 48.0 & 5.92 \\
T10E10 & 2.68 & 41.0 & 6.53 \\
\hline
\end{tabular}




\section{C.7 PN Index Revised}

The general procedure for the revised PN index involves adjusting the Reid Vapour Pressure and the DBE score of the base gasoline. This was done using the following criteria: runs with higher isobutylene concentrations had the vapour pressure of the base gasoline fraction artificially increased, while runs with higher toluene concentrations had their DBE scores artificially increased. The DBE scores were artificially modified by changing the $\mathrm{H}: \mathrm{C}$ atomic ratios used to calculate the molecular formula. Changes to the Reid Vapour Pressure were performed by referencing typical vapour pressures for gasoline fuels, per the US EPA [96]. These adjusted base gasoline fuel properties are presented in Table C.4. Recall that Fuel A was found to have ethanol exhaust emissions equivalent to an E10 blend; therefore, its molecular formula derives from the atomic ratio ( $\mathrm{H}: \mathrm{C}=1.92: 1)$ for E10 from the US EPA [79].

\section{C.7.1 Fuel Properties for Revised PN Index}

Table C.4: Base gasoline fuel properties used for the corrected PN indices.

\begin{tabular}{lccccc}
\hline & \multicolumn{5}{c}{ Base Gasoline $(i)$} \\
\cline { 2 - 6 } Property & Fuel A-1 & Fuel A-2 & Fuel B-1 & Fuel B-2 & Fuel B-3 \\
\hline Density $(\mathrm{kg} / \mathrm{L})$ & & & 0.77 & & \\
Molecular Weight $(\mathrm{kg} / \mathrm{kmol})$ & 100 & 100 & 105 & 105 & 105 \\
Molecular Formula $(\mathrm{C}, \mathrm{H})$ & $8,15.36$ & $8,15.36$ & $8,14.8$ & 8,16 & $8,15.36$ \\
DBE+1 & 2.32 & 2.32 & 2.6 & 2 & 2.32 \\
Reid Vapour Pressure (psi) & 9 & 8 & 7.8 & 9 & 9 \\
Reid Vapour Pressure (kPa) & 62.06 & 55.16 & 53.78 & 62.06 & 62.06 \\
\hline
\end{tabular}

Description of the fuels in Table C.4:

- Fuel A-1 represents a spring blend of Fuel A (higher vapour pressure)

- Fuel A-2 represents a summer blend of Fuel A (lower vapour pressure)

- Fuel B-1 has the same properties as gasoline in Table C.2. These are the baseline properties for Fuel B.

- Fuel B-2 has a higher vapour pressure and lower DBE score than Fuel B-1. This represents cases where tests showed high isobutylene and low toluene exhaust concentrations.

- Fuel B-3 has the same vapour pressure as Fuel B-2 but a higher DBE score. This represents cases where isobutylene exhaust concentrations were high, and toluene concentrations were moderately high. 


\section{C.7.2 Calculation of Revised PN Index}

Table C.5 contains the calculated PN indices for the different test fuels presented in Figure 6.28. The same procedure presented in the above discussion (Section C.6.2) was applied here to obtain these calculated values. The properties for gasoline listed in Table C.4 were used along with the properties for toluene and ethanol in Table C.2 for the relevant fuel blends.

Table C.5: Calculated PN indices.

\begin{tabular}{ccccc}
\hline Test Fuel & Base Gasoline & DBE+1 & DVPE $[\mathrm{kPa}]$ & PN Index \\
\hline Fuel A - Spring & Fuel A-1 & 2.32 & 62.06 & 3.74 \\
Fuel A - Summer & Fuel A-2 & 2.32 & 55.16 & 4.21 \\
Fuel B - June & Fuel B-1 & 2.6 & 53.78 & 4.83 \\
E0 & Fuel B-2 & 2 & 62.06 & 3.22 \\
E10 & Fuel B-3 & 2.19 & 52.54 & 4.16 \\
E30 & Fuel B-1 & 2.12 & 34.8 & 6.09 \\
E0 Return & Fuel B-1 & 2.6 & 53.78 & 4.83 \\
T10 & Fuel B-3 & 2.58 & 55.24 & 4.68 \\
T10E10 & Fuel B-1 & 2.68 & 41.0 & 6.53 \\
\hline
\end{tabular}




\section{Appendix D}

\section{Chapter 7 Supplementary Start Data}

\section{D.1 Cold Start: Particle Size Distributions}

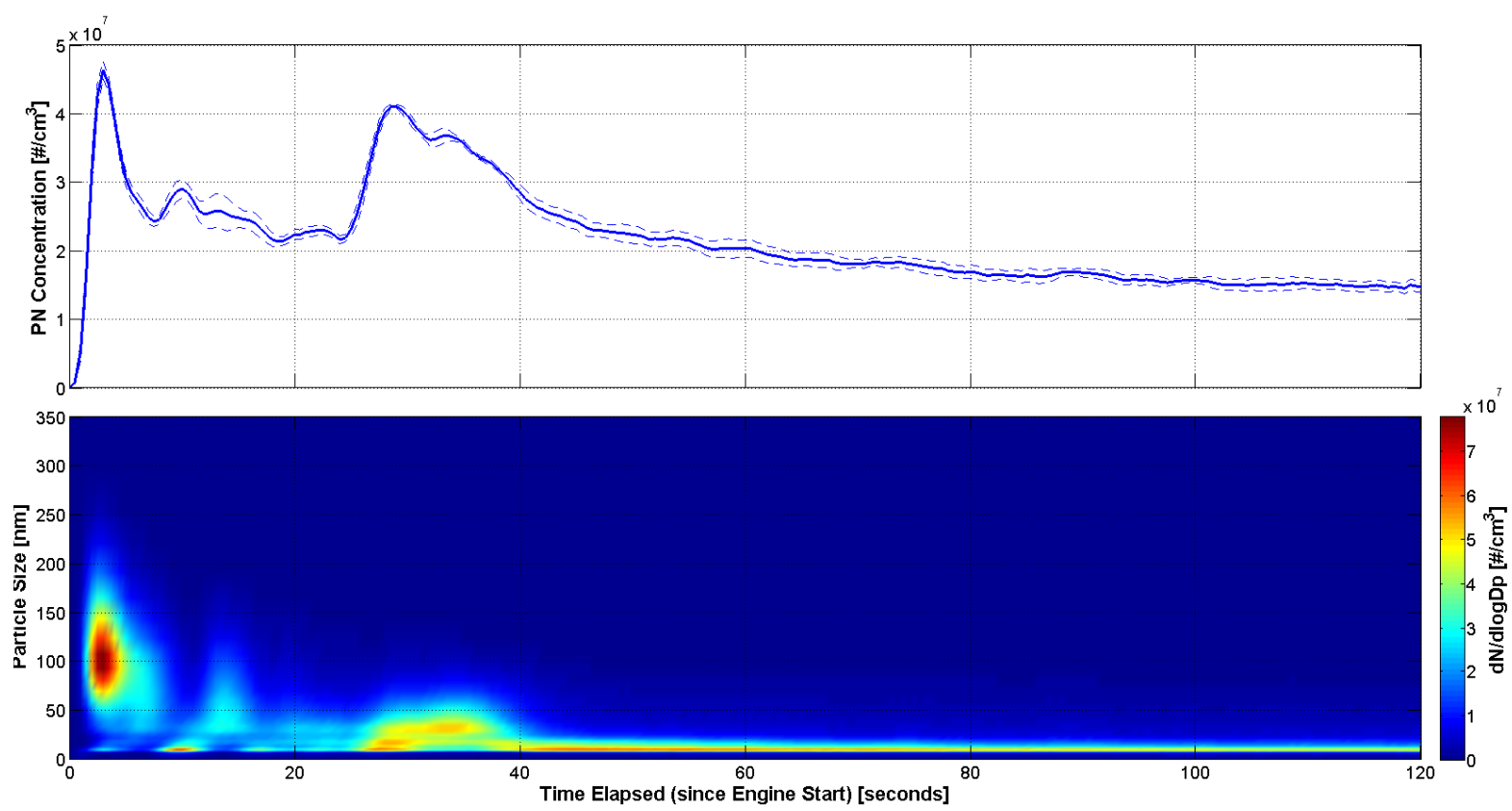

Figure D.1: Average cold start PN concentration (top) and particle size distribution (bottom) with time for E10 fuel. Dashed lines in top figure indicate standard error. 


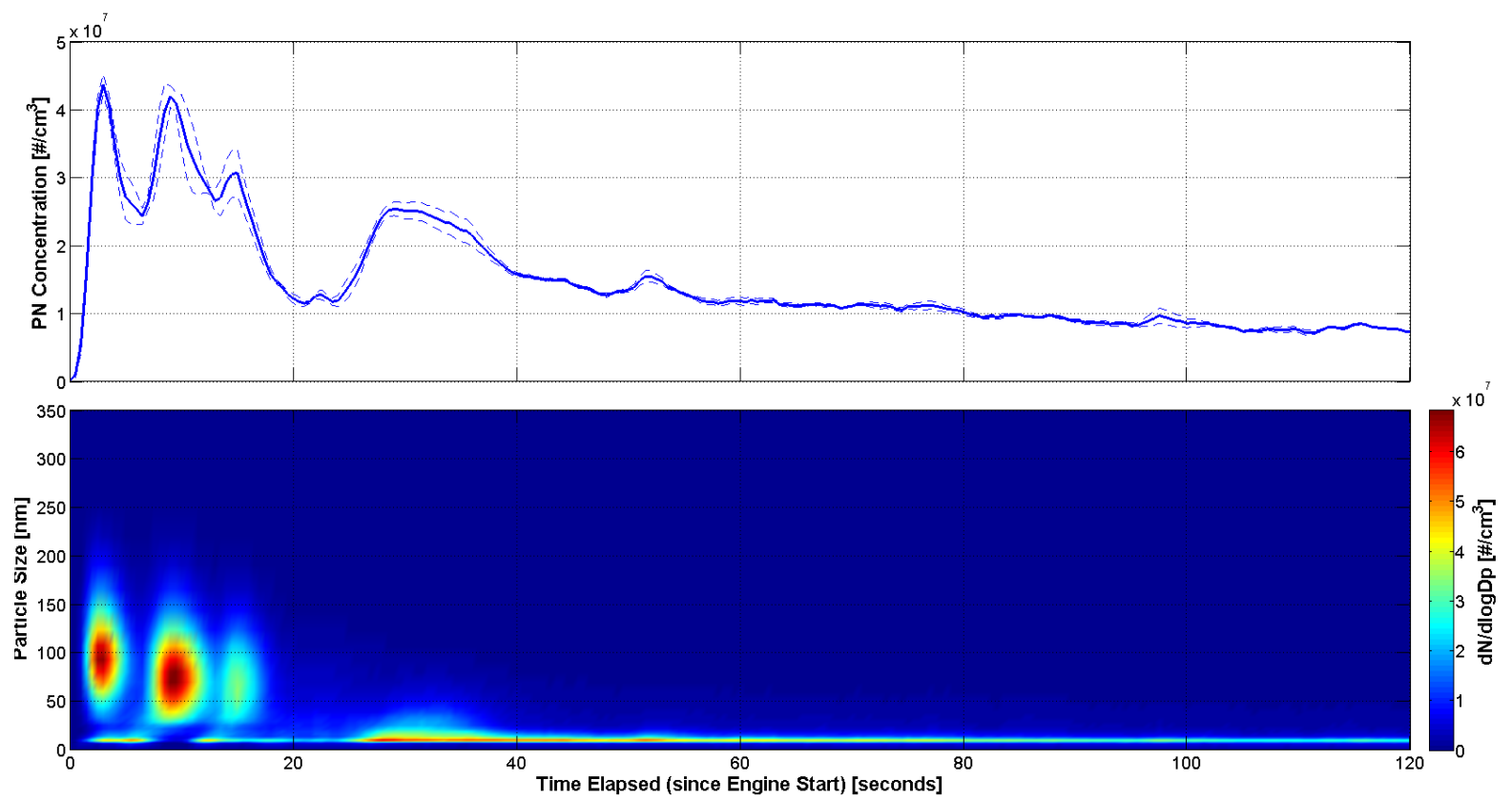

Figure D.2: Average cold start PN concentration (top) and particle size distribution (bottom) with time for E30 fuel. Dashed lines in top figure indicate standard error.
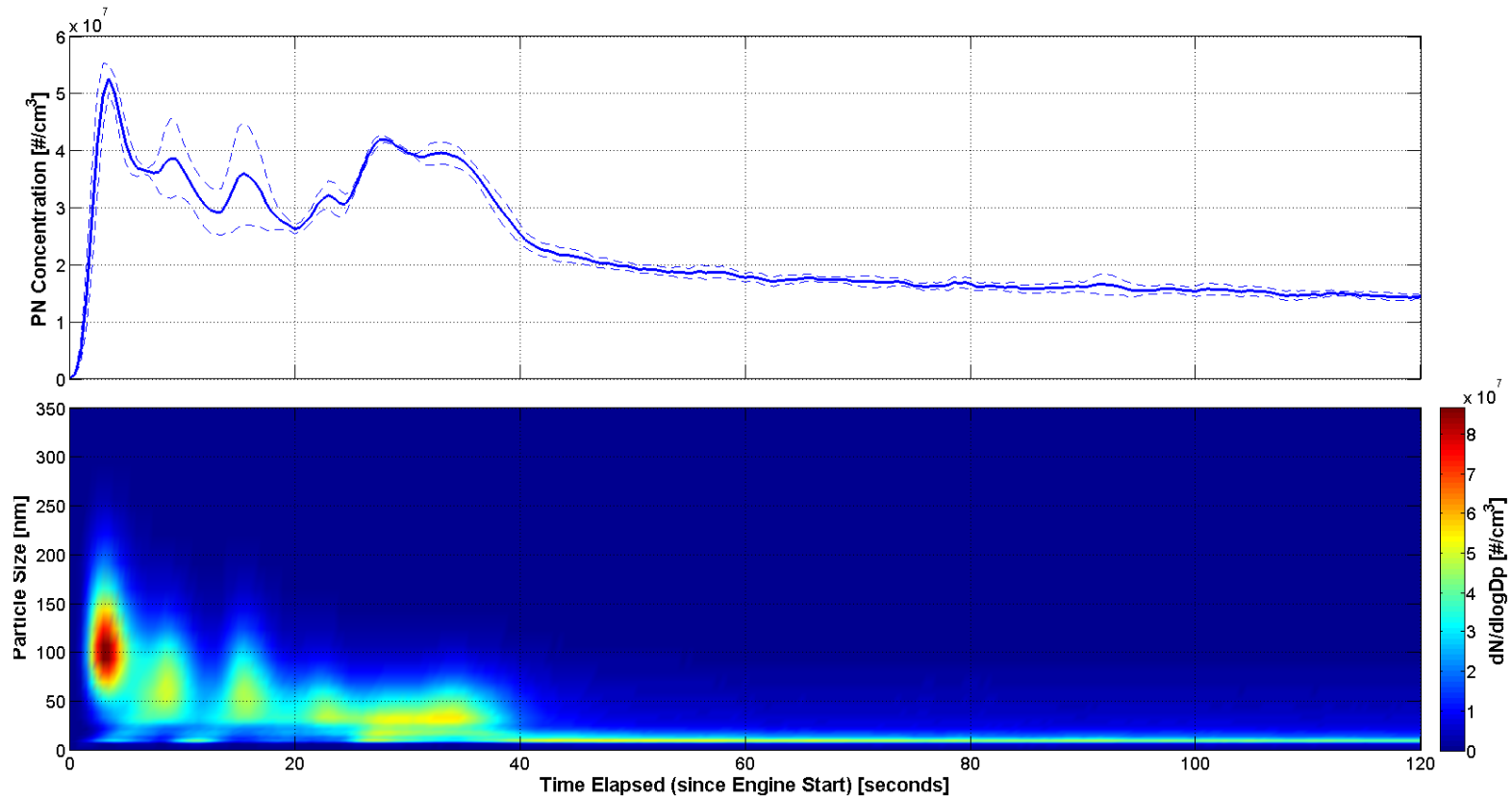

Figure D.3: Average cold start PN concentration (top) and particle size distribution (bottom) with time for E0 fuel (return test group). Dashed lines in top figure indicate standard error. 


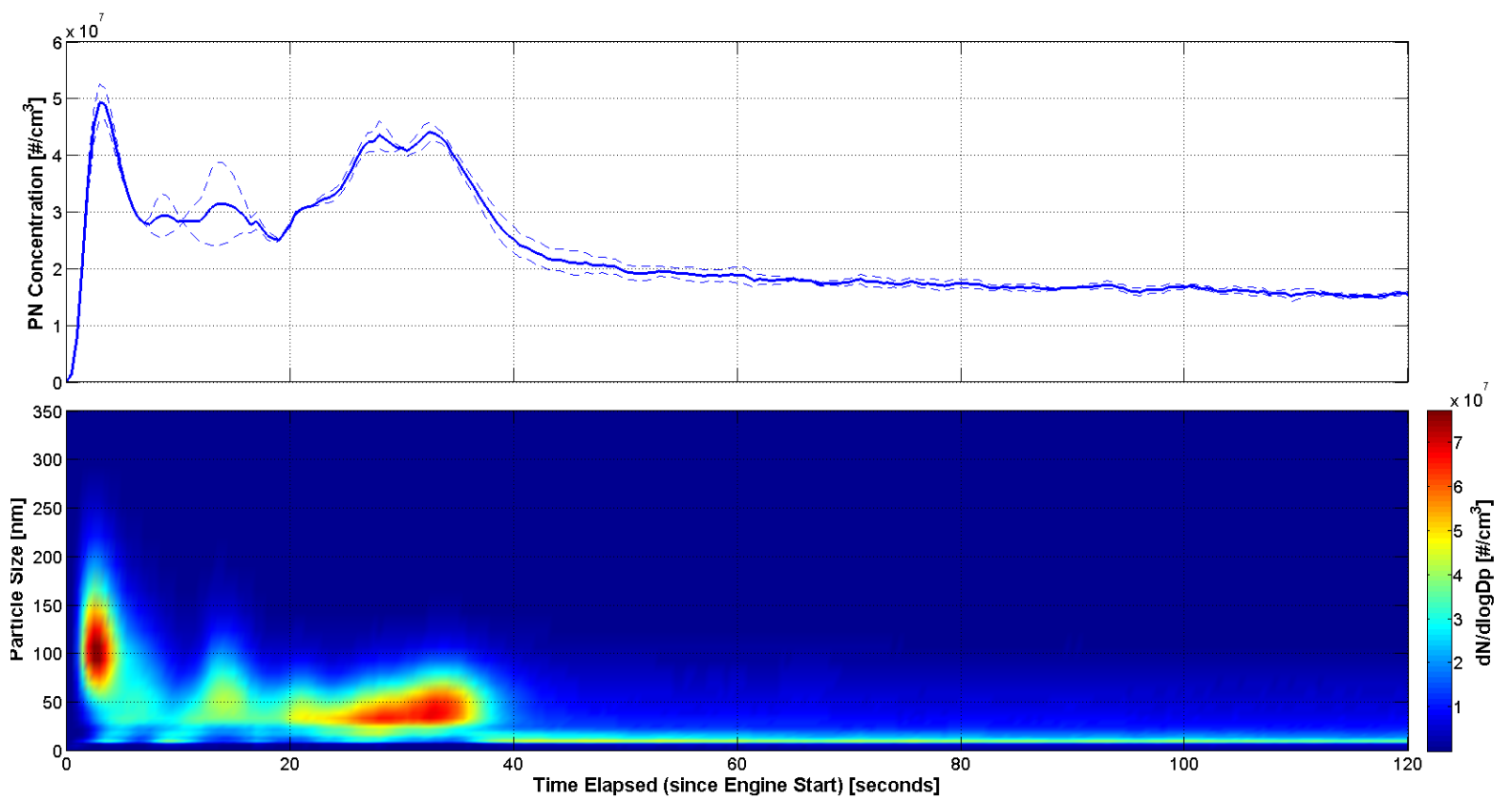

Figure D.4: Average cold start PN concentration (top) and particle size distribution (bottom) with time for T10 fuel. Dashed lines in top figure indicate standard error.
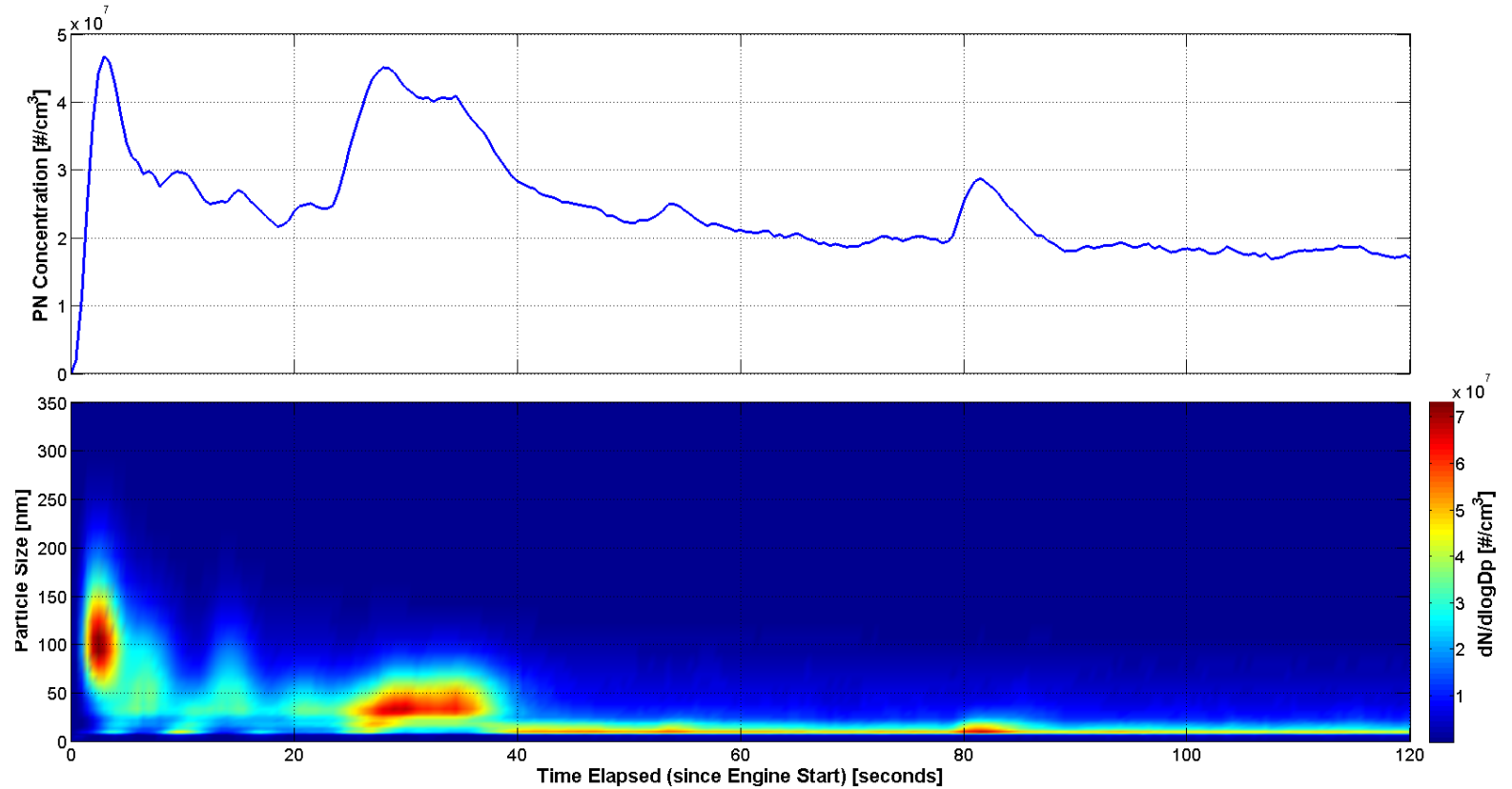

Figure D.5: Single cold start PN concentration (top) and particle size distribution (bottom) with time for T10E10 fuel. 


\section{D.2 Cold Start: Gaseous Emissions}

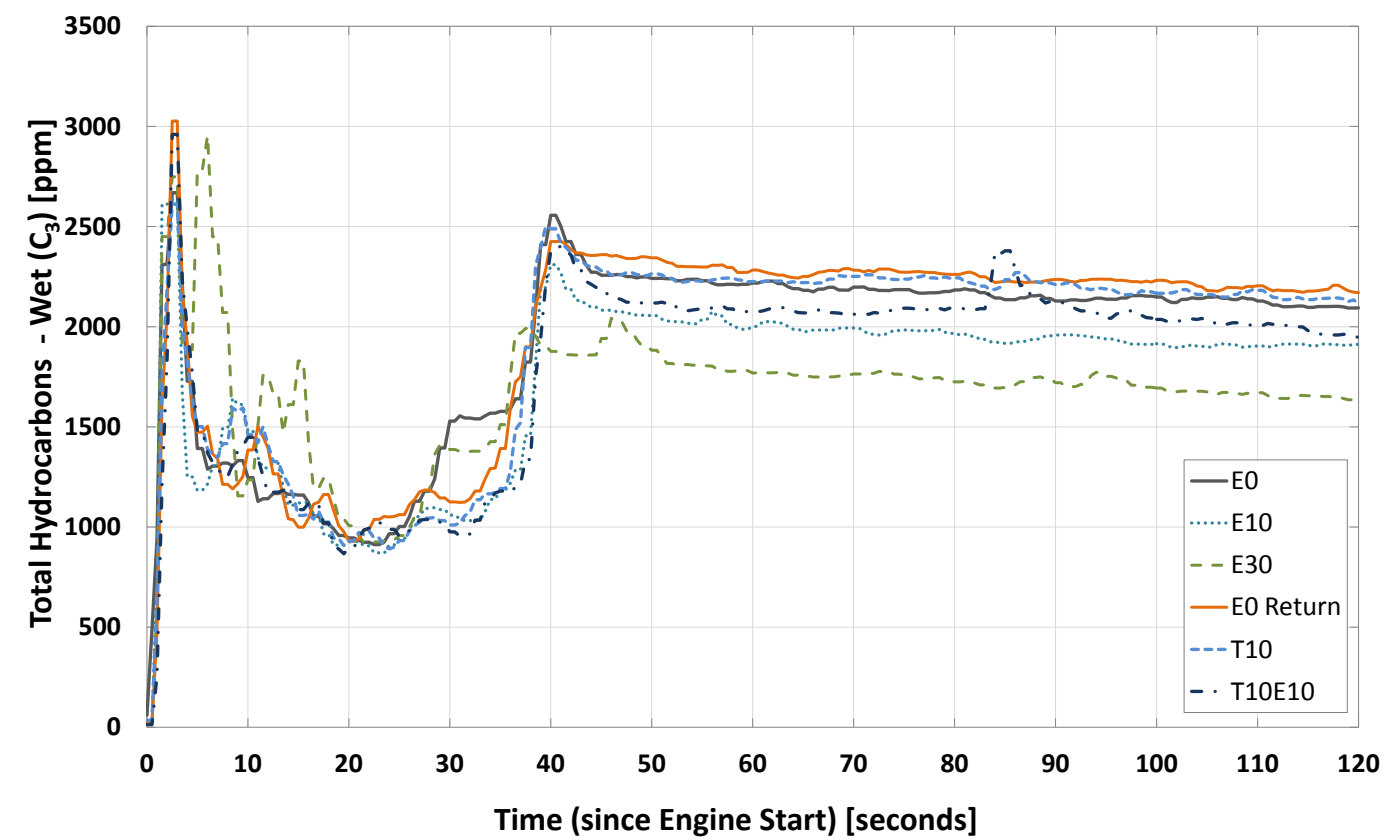

Figure D.6: Average cold start total hydrocarbon (THC) emission for different fuel blends.

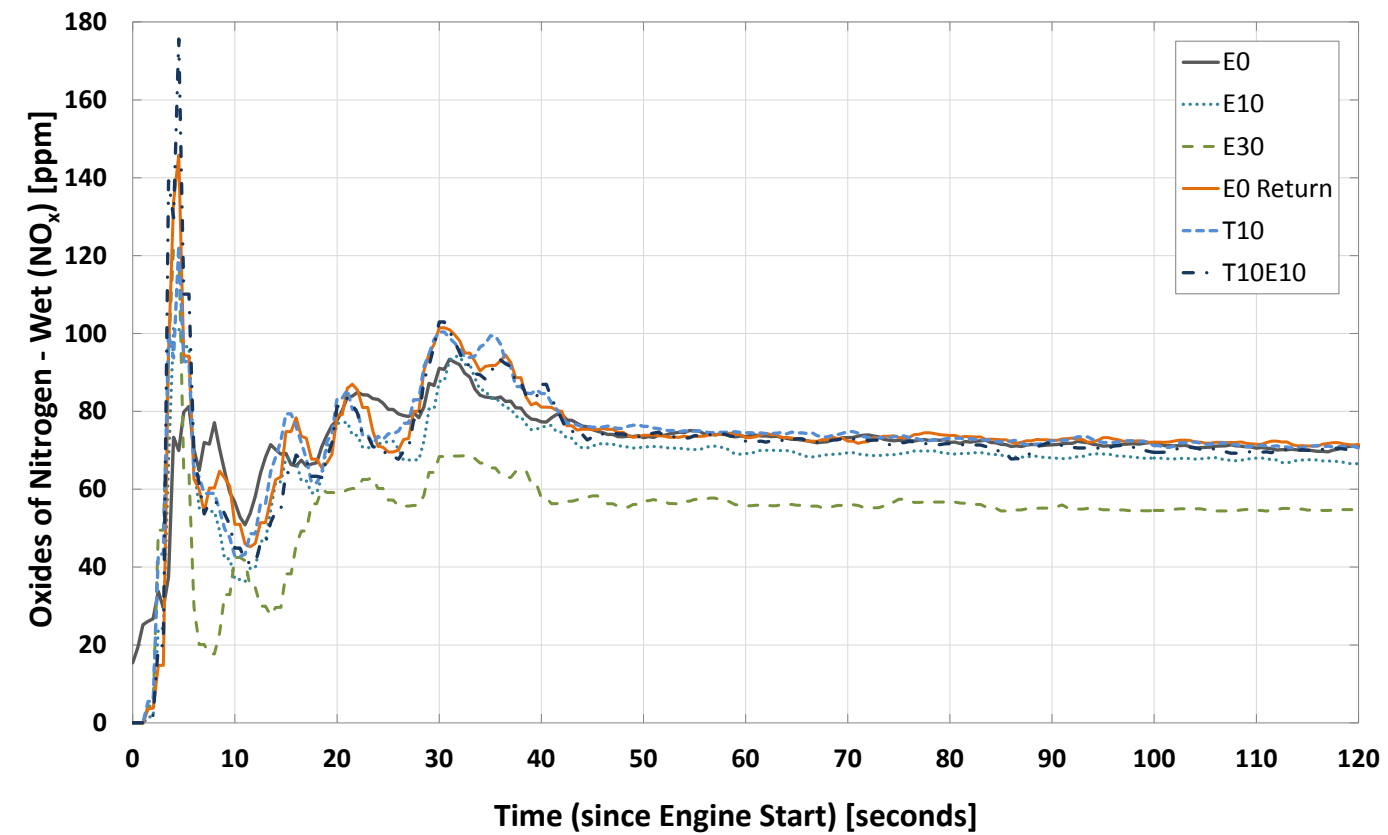

Figure D.7: Average cold start oxides of nitrogen $\left(\mathrm{NO}_{\mathrm{x}}\right)$ emissions for different fuel blends. 


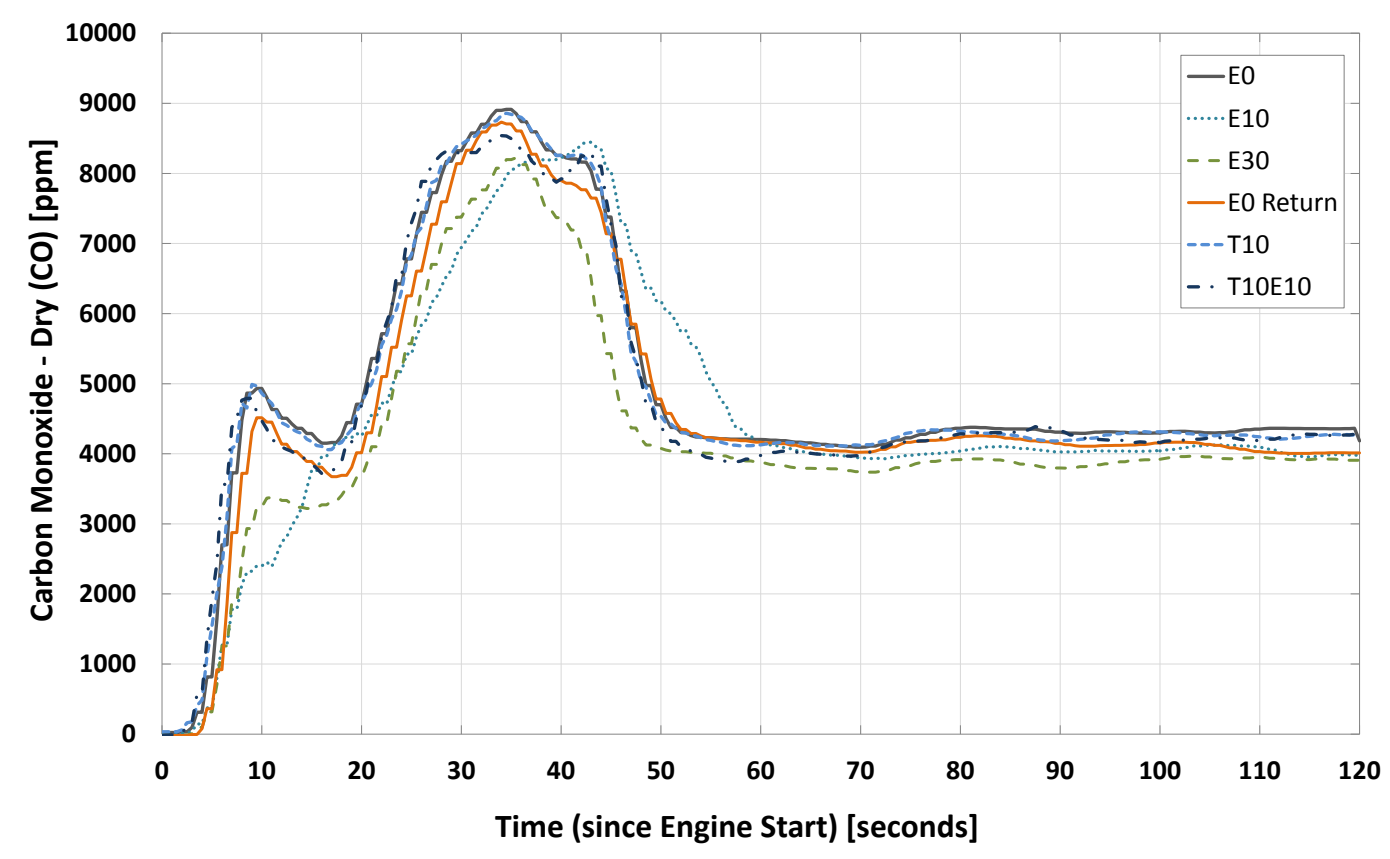

Figure D.8: Average cold start carbon monoxide (CO) emissions for different fuel blends.

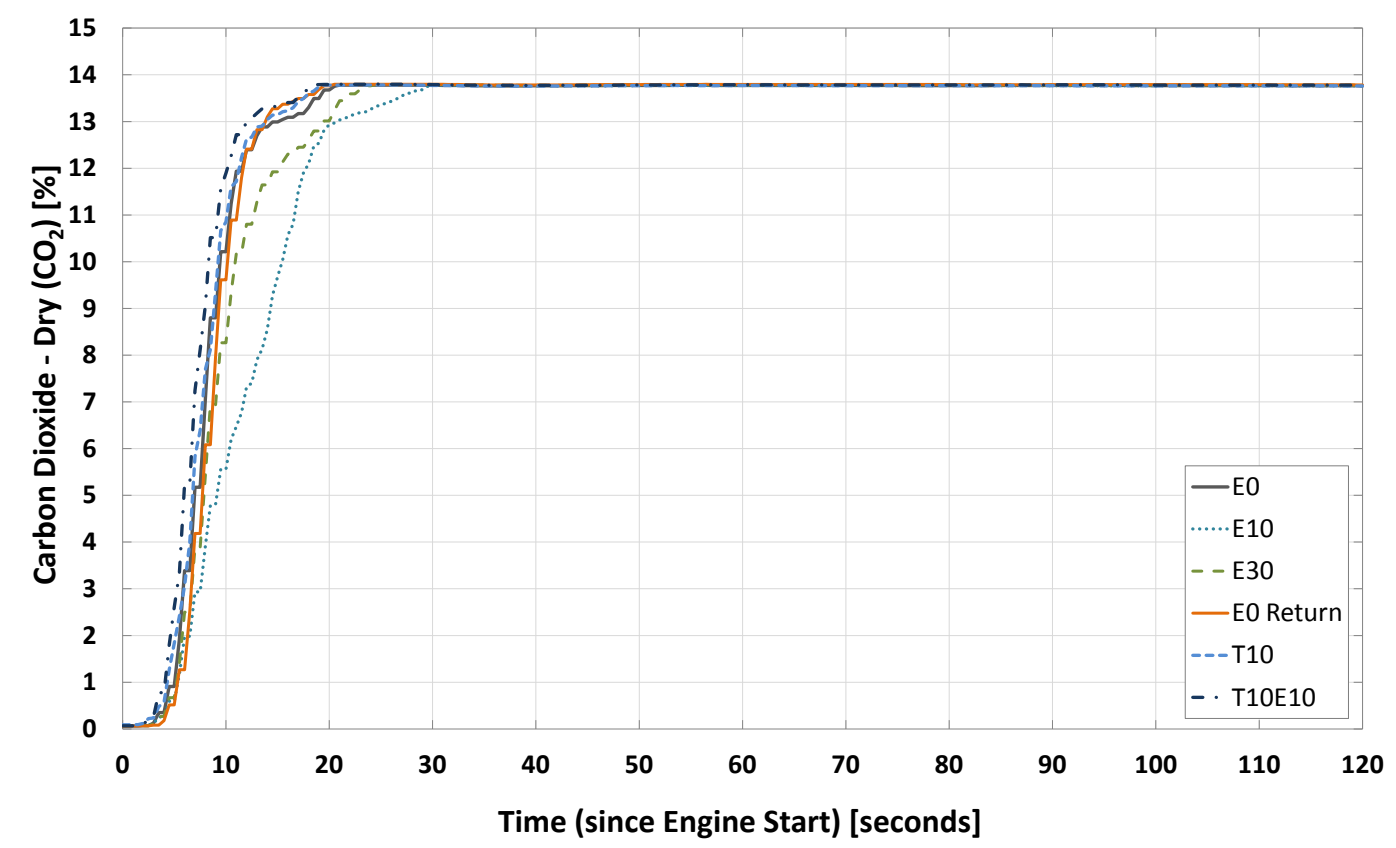

Figure D.9: Average cold start carbon dioxide $\left(\mathrm{CO}_{2}\right)$ emissions for different fuel blends. 


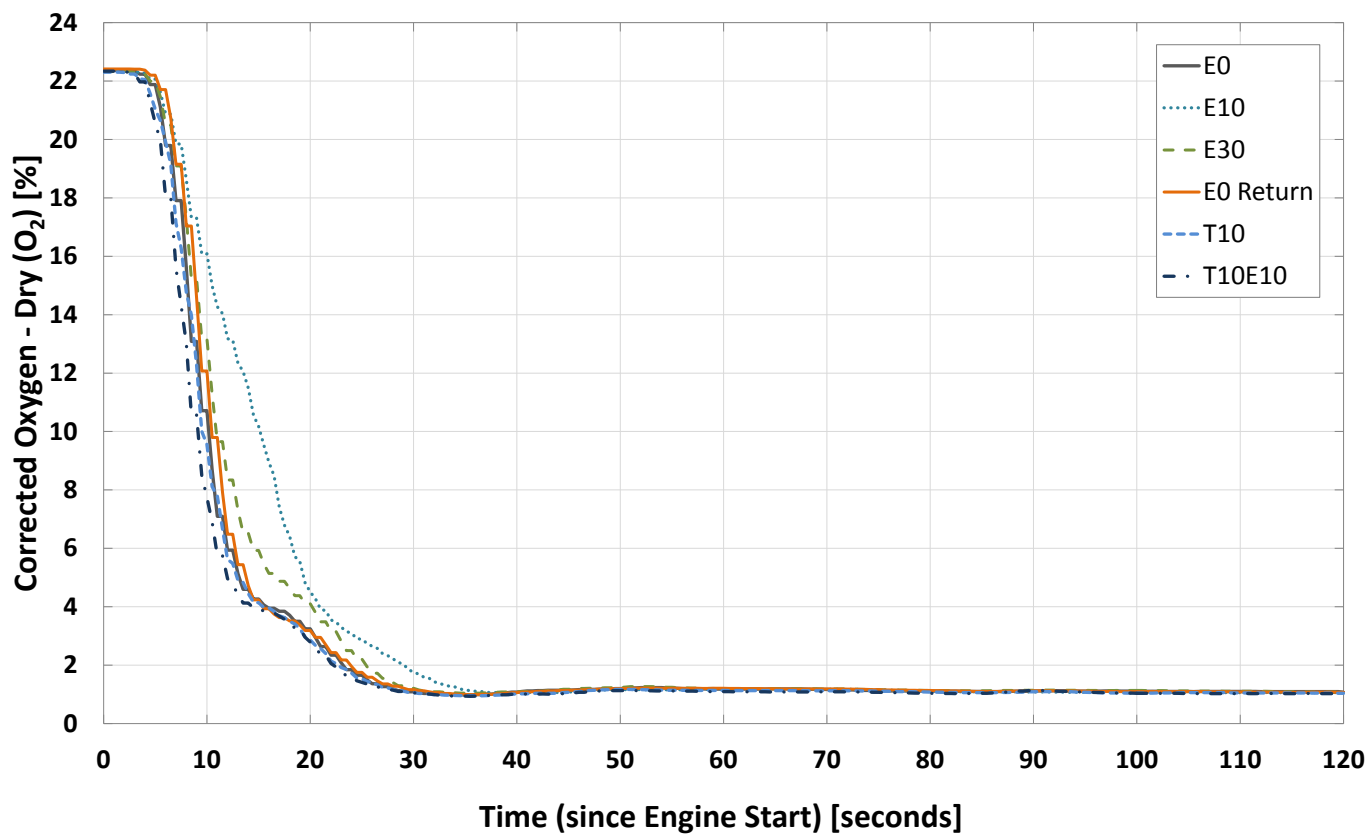

Figure D.10: Average cold start oxygen $\left(\mathrm{O}_{2}\right)$ emissions for different fuel blends. 


\section{D.3 Hot Start: Particle Size Distributions}

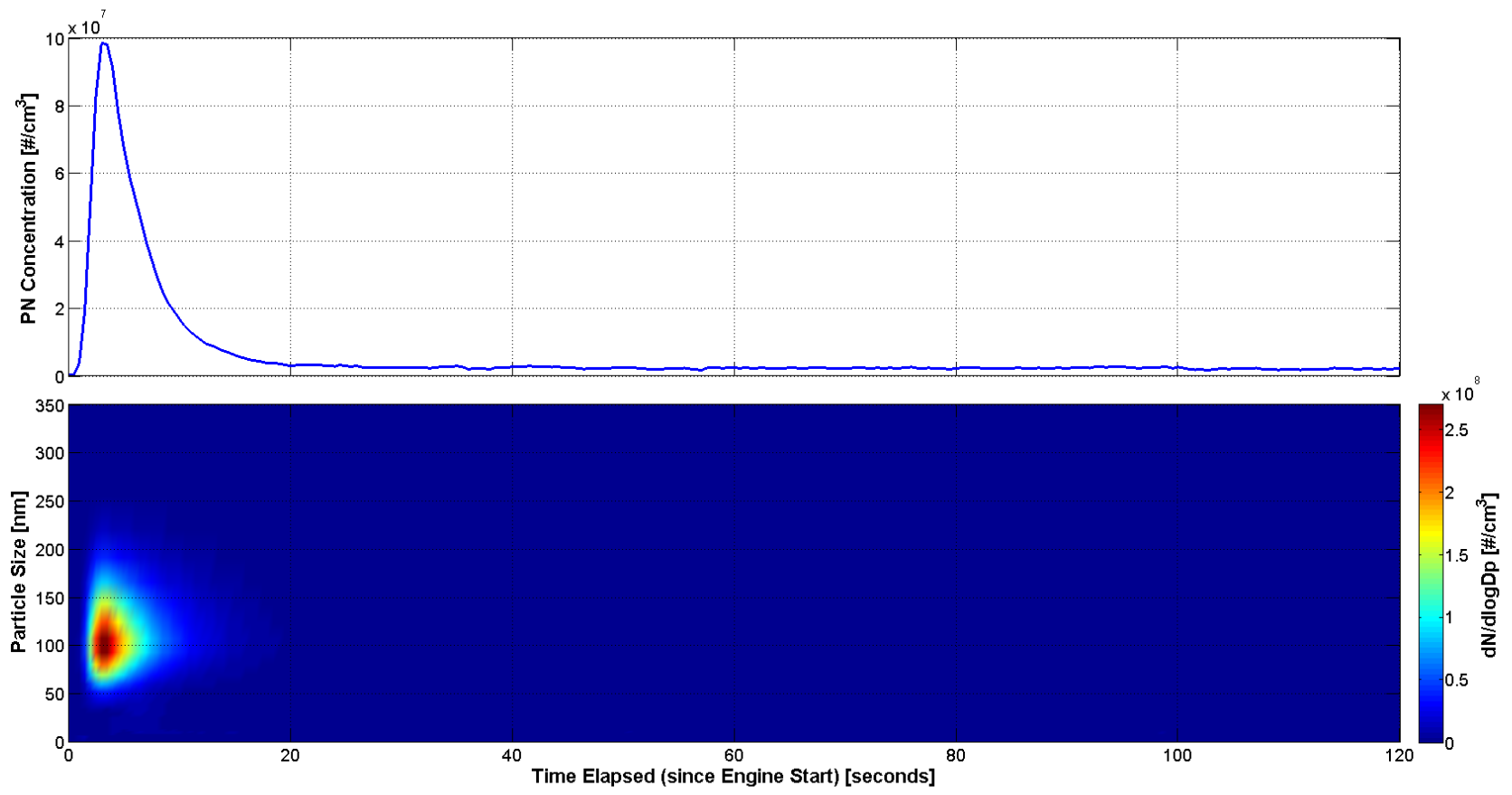

Figure D.11: Hot start PN concentration (top) and particle size distribution (bottom) with time for E10 fuel.
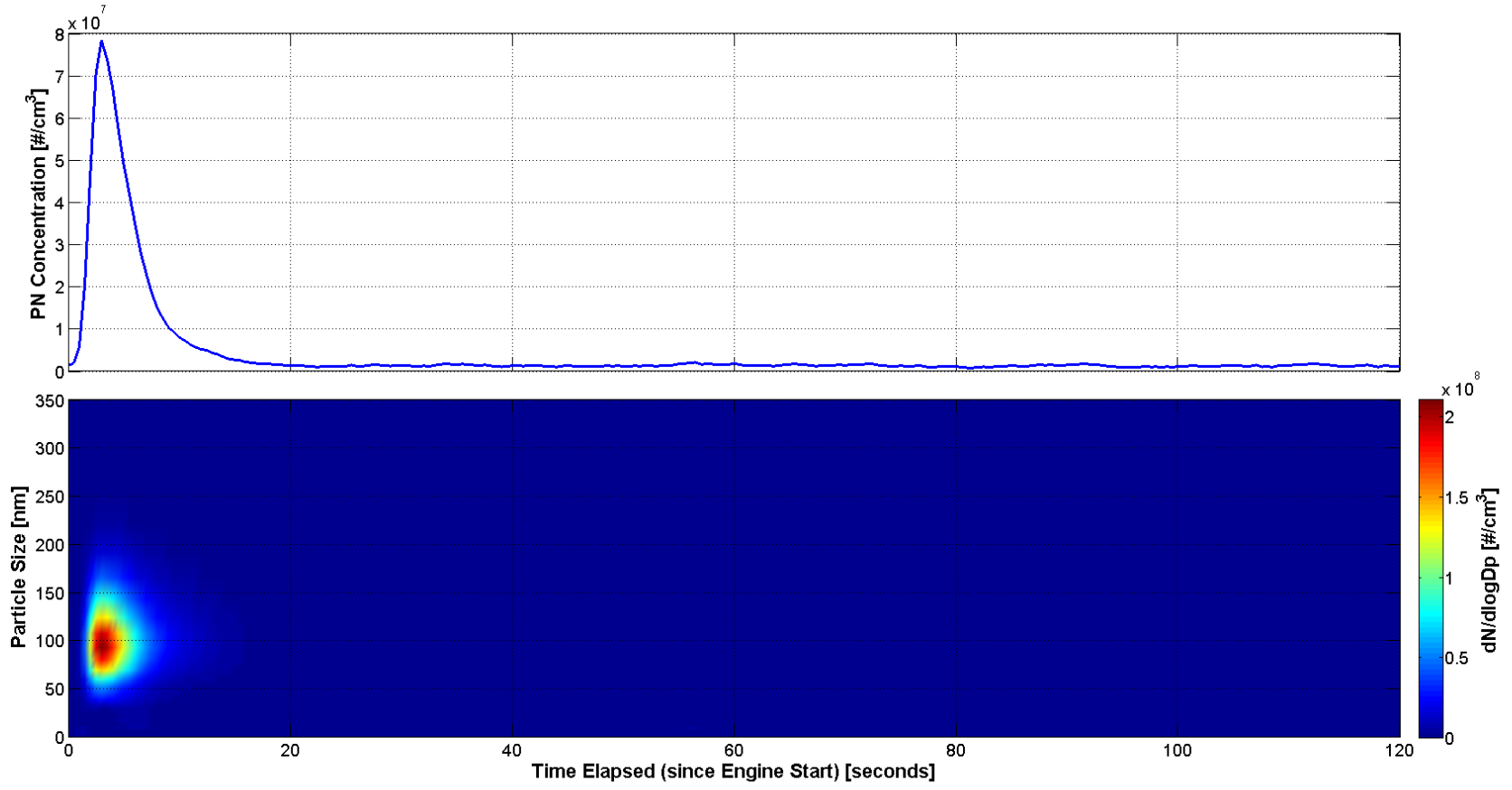

Figure D.12: Hot start PN concentration (top) and particle size distribution (bottom) with time for E30 fuel. 

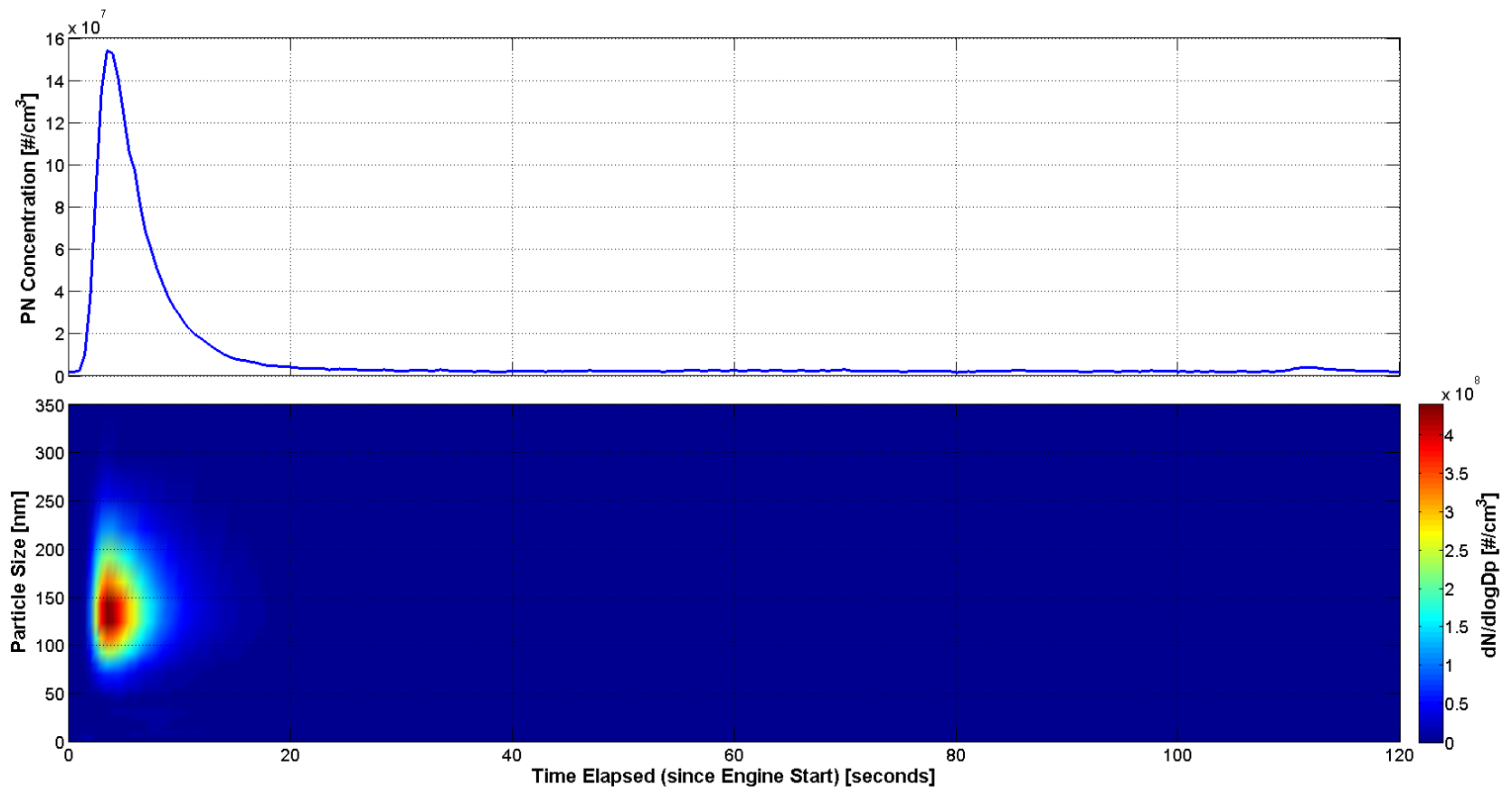

Figure D.13: Hot start PN concentration (top) and particle size distribution (bottom) with time for T10 fuel.
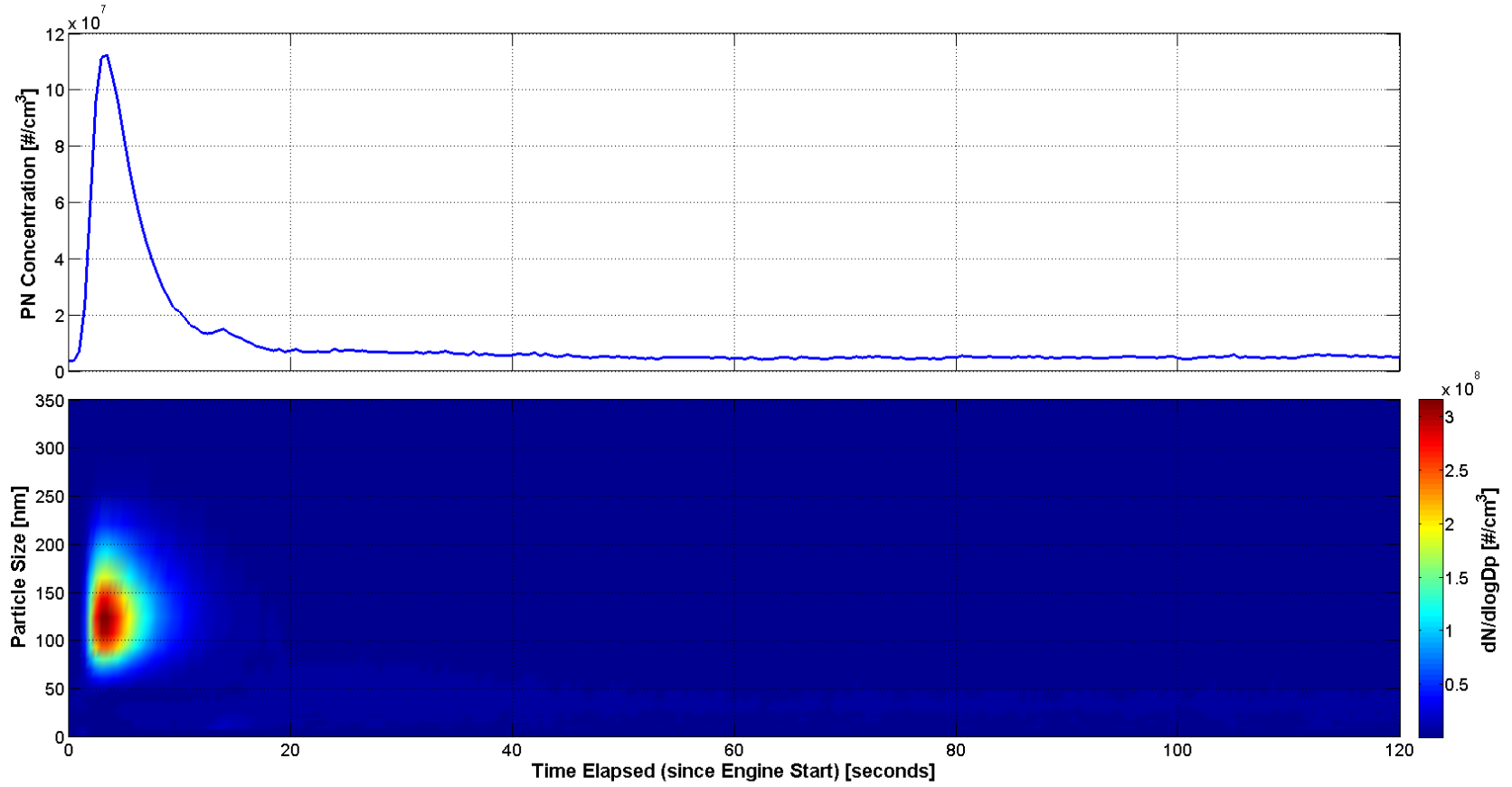

Figure D.14: Hot start PN concentration (top) and particle size distribution (bottom) with time for T10E10 fuel. 


\section{D.4 Hot Start Gaseous Emissions}

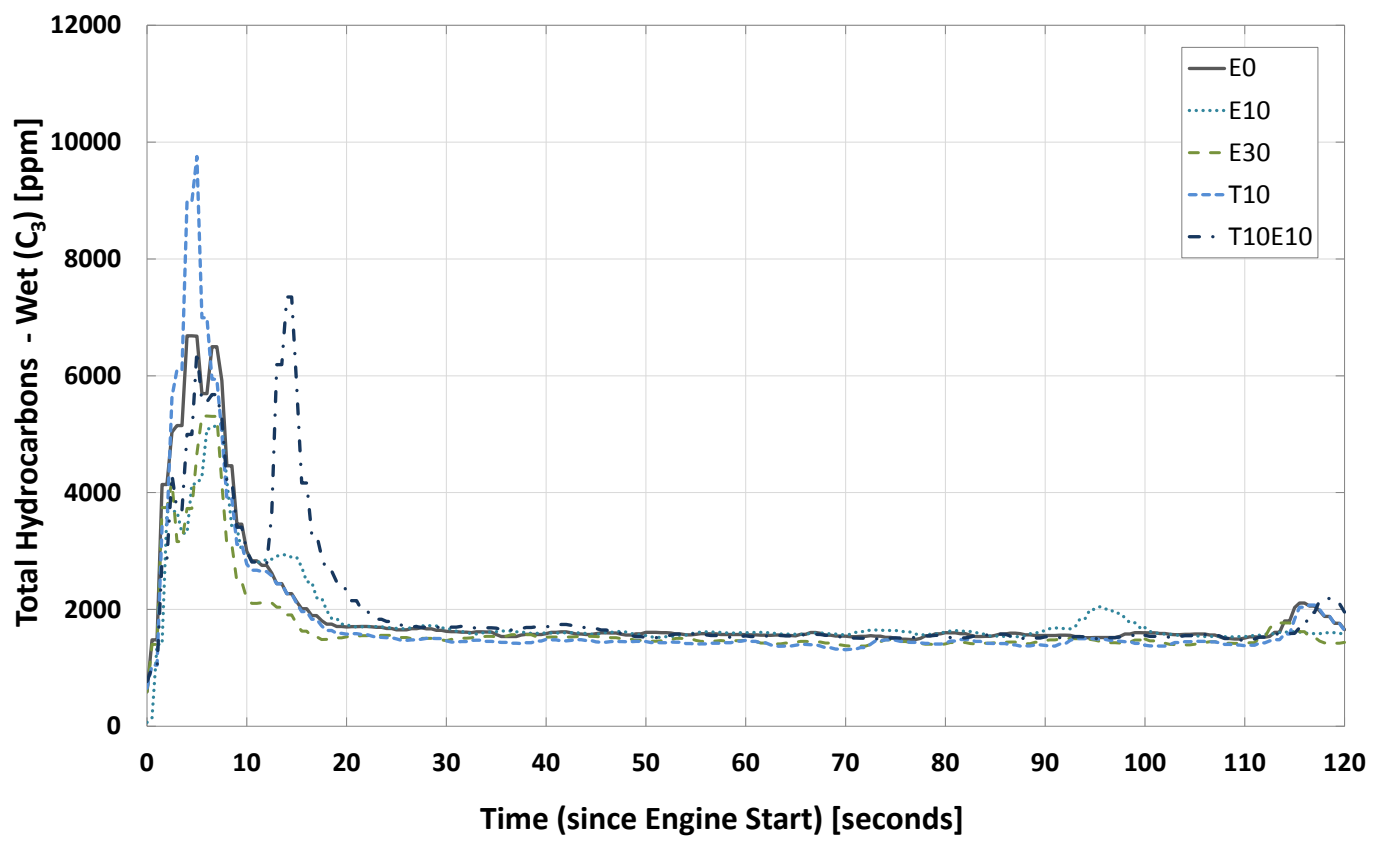

Figure D.15: Hot start total hydrocarbon (THC) emission for different fuel blends.

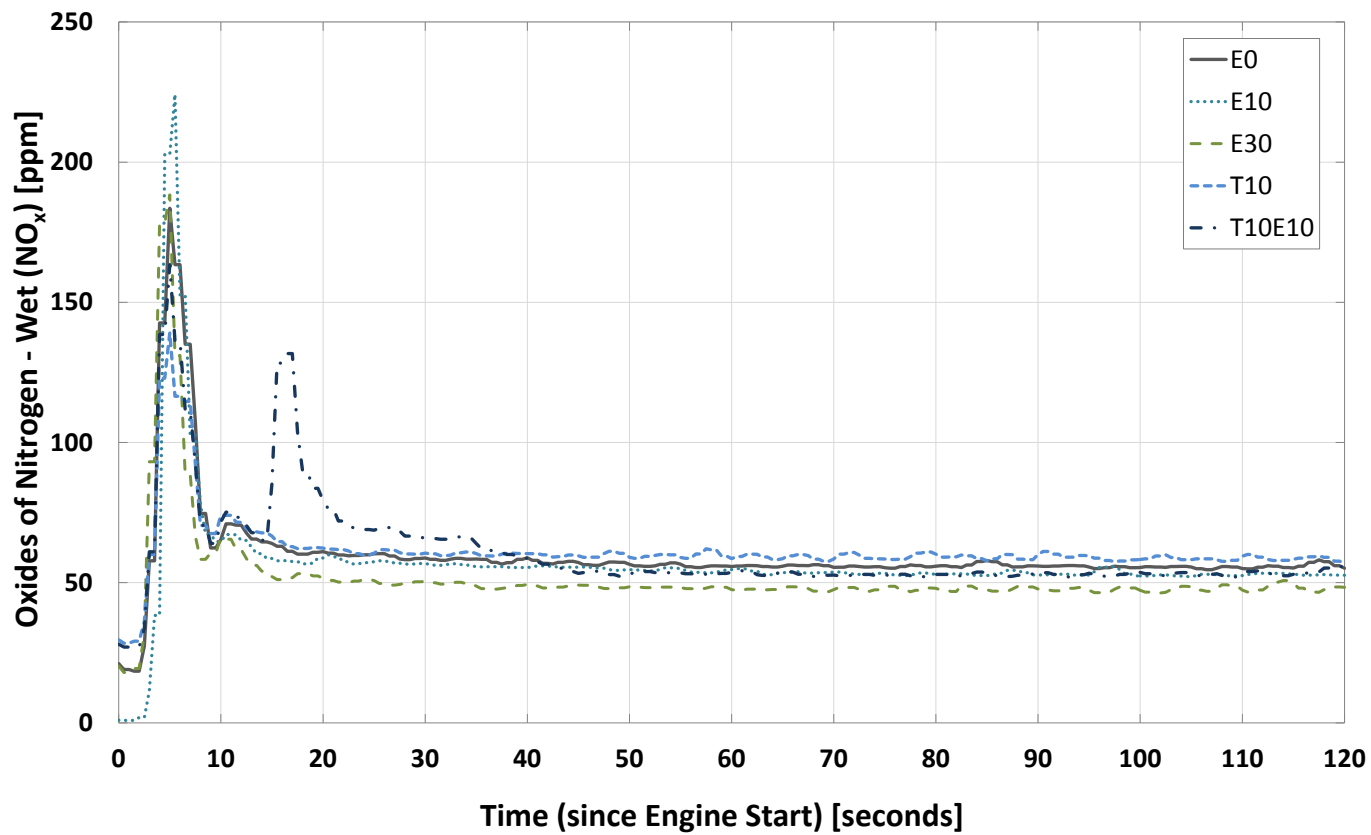

Figure D.16: Hot start oxides of nitrogen $\left(\mathrm{NO}_{\mathrm{x}}\right)$ emissions for different fuel blends. 


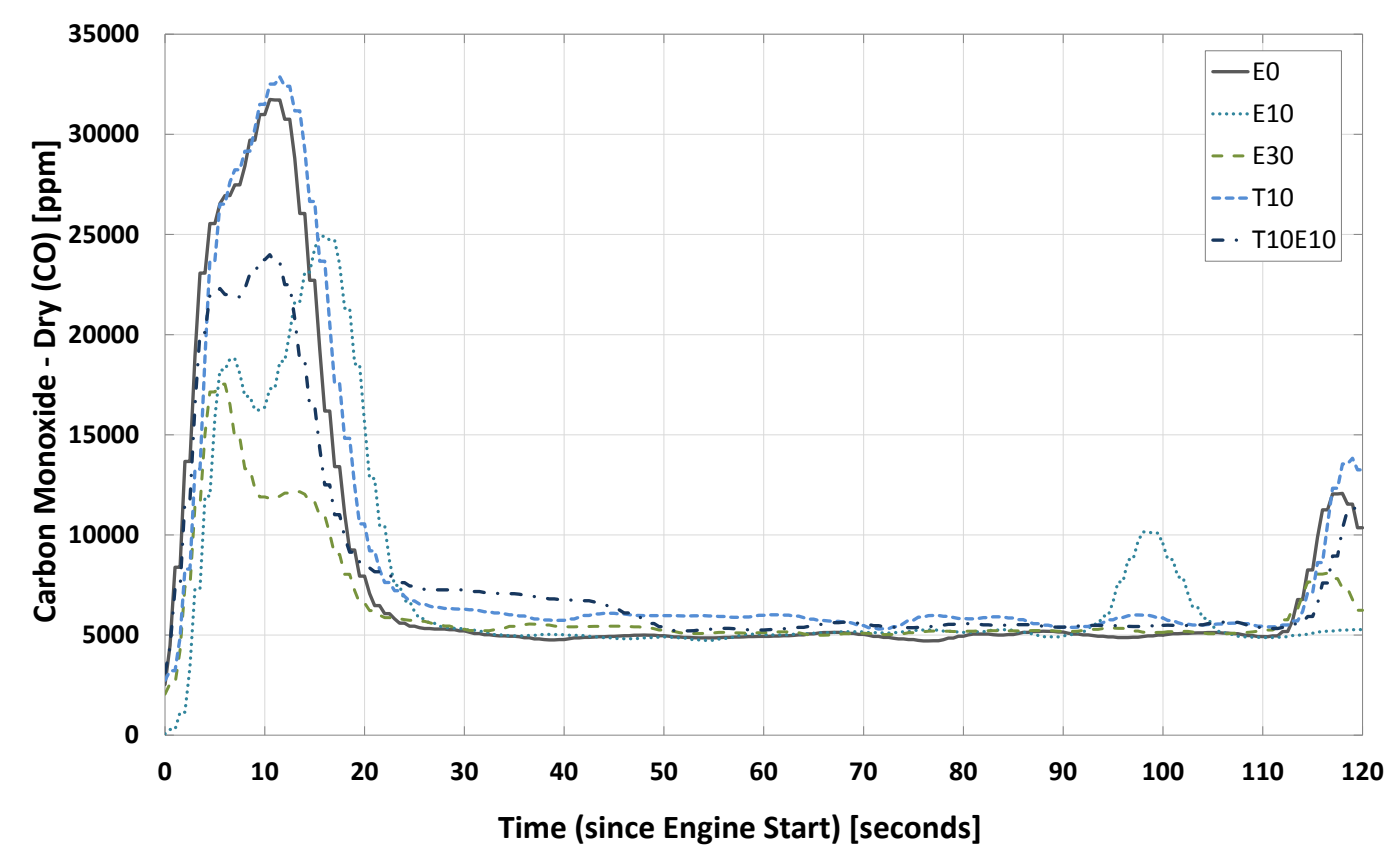

Figure D.17: Hot start carbon monoxide (CO) emissions for different fuel blends.

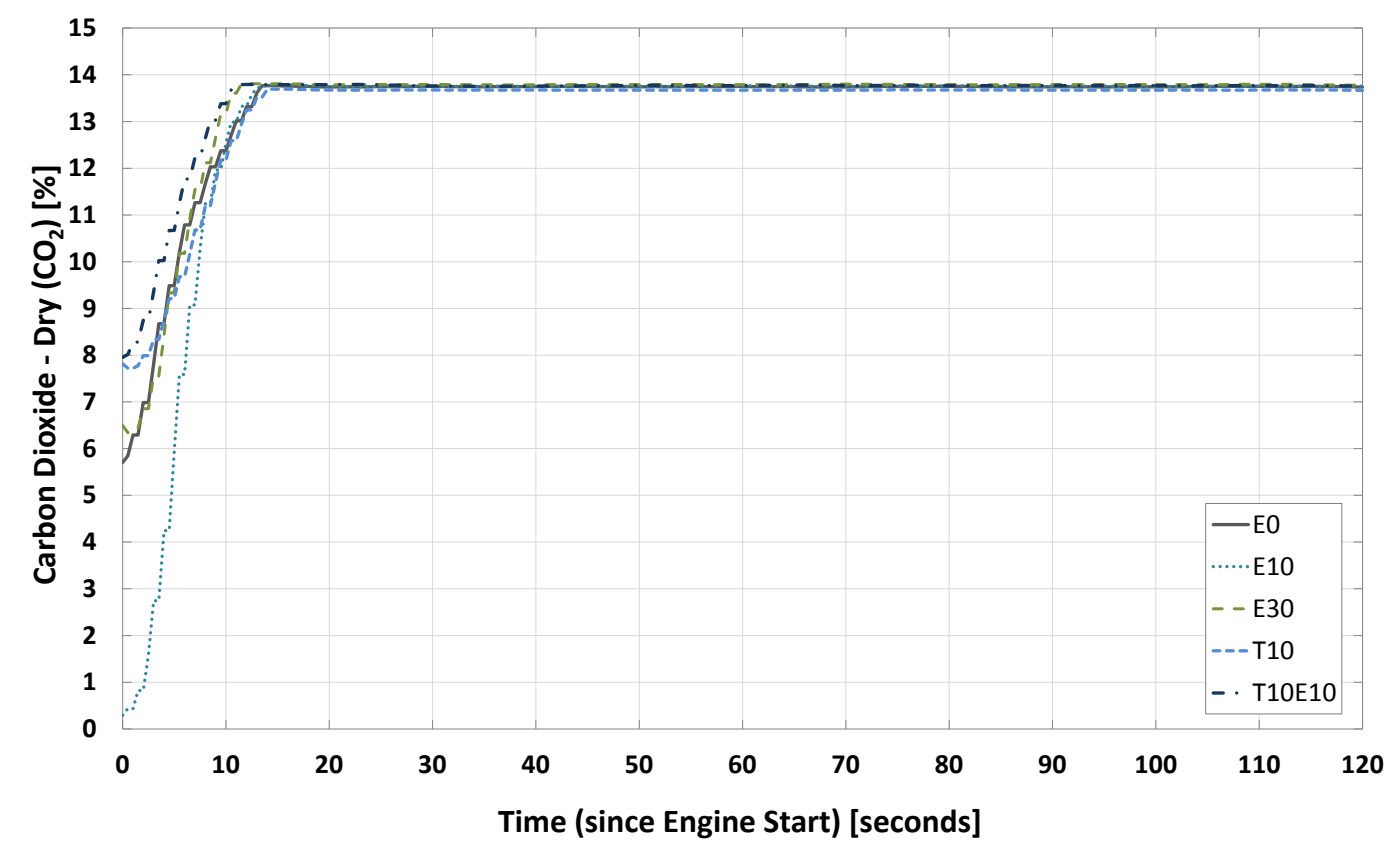

Figure D.18: Hot start carbon dioxide $\left(\mathrm{CO}_{2}\right)$ emissions for different fuel blends. 


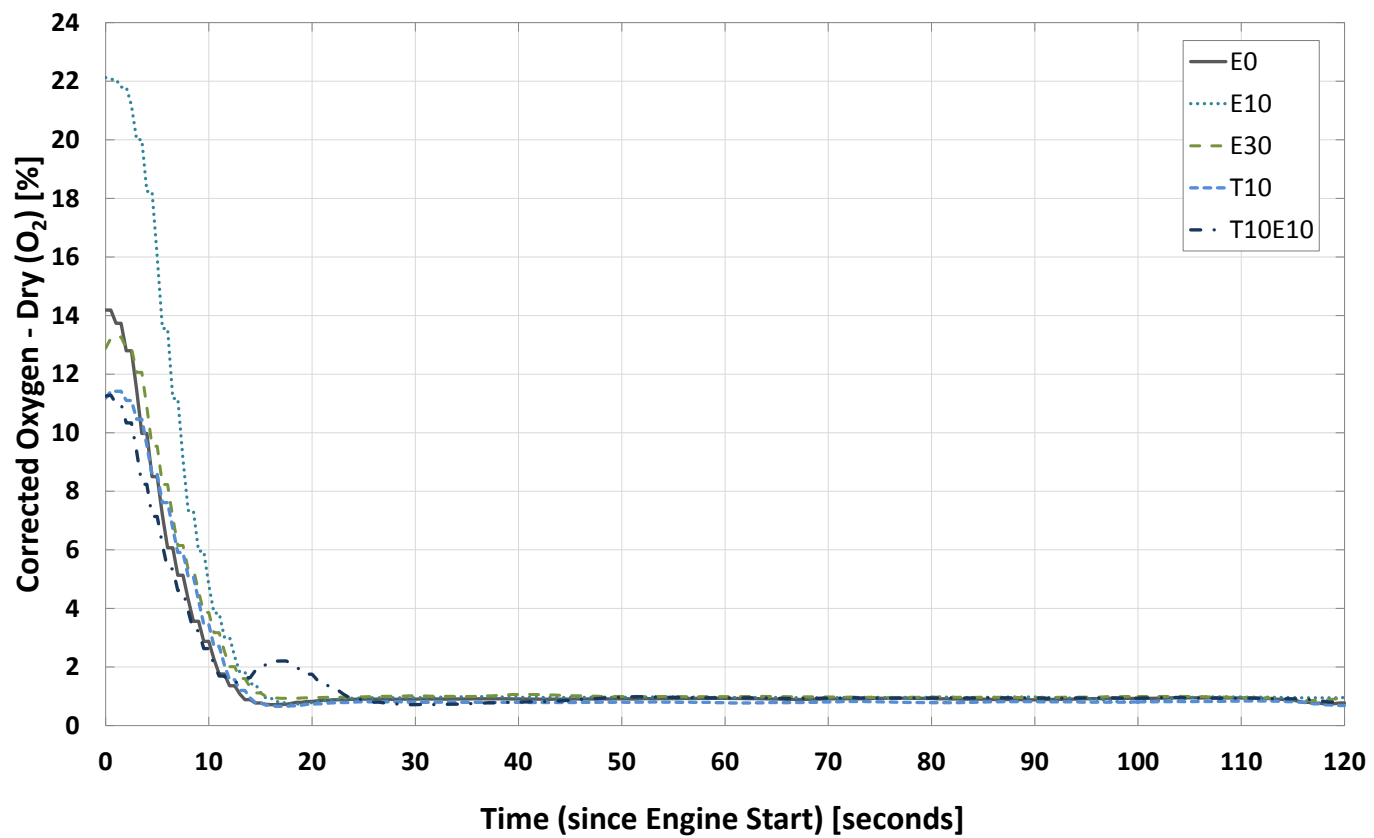

Figure D.19: Hot start oxygen $\left(\mathrm{O}_{2}\right)$ emissions for different fuel blends. 